

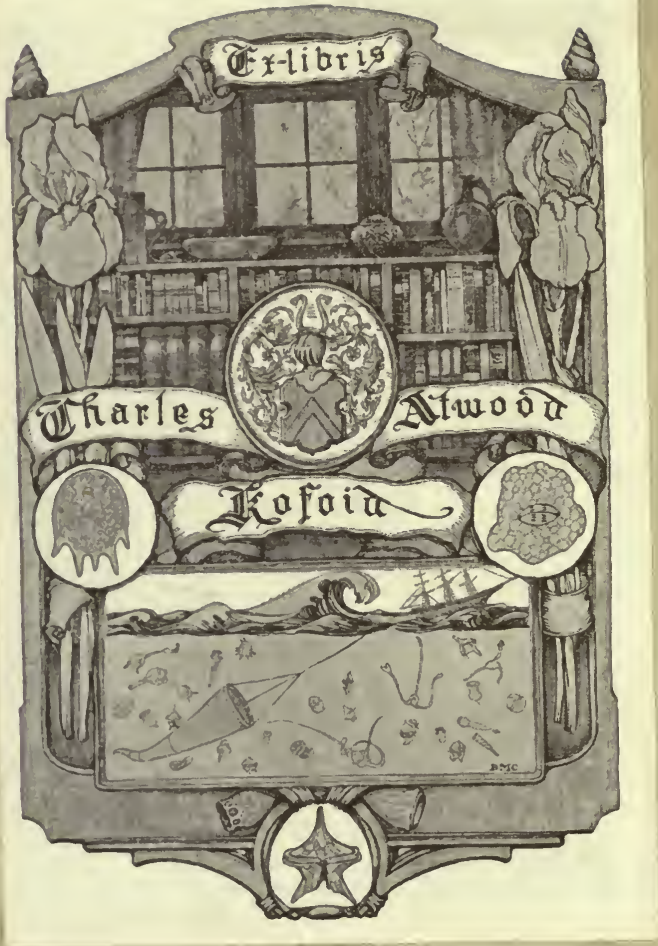




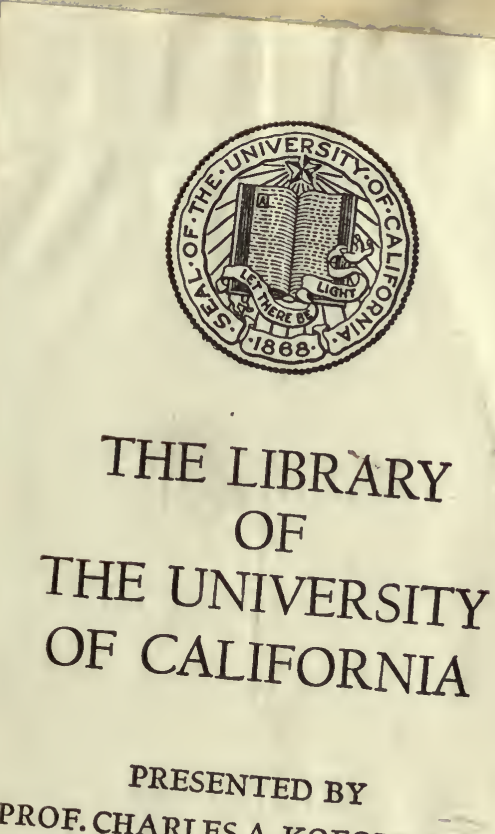
PROF. CHARLES A. KOFOID AND MRS. PRUDENCE W. KOFOID 



\section{By the sume Author.}

\section{MEDICAL NOTES AND REFLECTIONS.}

Third Edition, revised throughout; with some Additions. 8vo. 18s.

\section{Contents.}

On Medical Evidence.

On IIereditary Disease.

Method of Inquiry as to Contagion.

On Diseases commonly occurring but once in Life.

On the Connexion and Classification of certain Diseases.

On disturbed Balance of Circulation, and Metastasis of Disease.

On the Influence of Weather in relation to Disease.

On Diet, and Digorders of Digestion.

On Gout as a Constitutional Disorder.

On Morbid Actions of Intermittent kind.

On the Medical Treatment of Old Age.

On the Epidemic Influenzas of late years.

On Prognosis, as a part of Practice.

On Pain, as a Symptom of Disease.

On points where a Patient may judge for himself.

On Methods of Prescription.
On Internal Hæmorrhages and Morbid Secretions as the Subjects of Medical Treatment.

On some supposed Diseases of the Spine.

On Hypochondriasis.

On the Exercise of Respiration.

On some Polnts in the Pathology of the Colon.

On the Abuse of Purgative Medicines.

On Blceding in Affections of the Brain.

On the Use of Emetics.

On the Uses of Diluents.

On Sudoriflc Medicines.

On the Use of Opiates.

On Mercurial Medicines.

On the Use of Digitalis.

On Antimonial Medicines.

On the Hypothesis of Animalenle Life as a Cause of Disease.-On Cholera.

Conclusion.

\section{CHAPTERS ON MENTAL PHYSIOLOGY,}

Founded chiefly on Chapters contained in 'Medical Notes and Reflections.' Second Edition revised and enlarged Post 8vo, 8s. $6 d$.

\section{Contents.}

On Sleep.

On the Relations of Dreaming, Insanity, \&c. Effects of Mental Attention on Bodily Organs.

On Mental Consciousness, in its Relation to Time and Succession.

On Time, in further Relation to Mental Functions.

On the Memory, as affected by $\mathbf{A g e}$ and Disease.
On the Brain as a Double Organ.

On Phrenology.

On Instincts and Habits.

On the Present State of Inquiry into the Nervous System.

On Medical Evidence. 
- Digitized by the Internet Archive in 2007 with funding from Microsoft Corporation 


\section{ESSAYS}

FROM

\section{THE EDINBURGH AND QUARTERLY REVIEWS.}


LONDON

PRINTED BY SPOTTISWOODE AND CO.

NEW-STREET SQUARE 


\title{
ESSAYS
}

ON

\section{SCIENTIFIC AND OTHER SUBJECTS}

\author{
CONTRIBCTED TO THE
}

EDINBURGH AND QUARTERLY REVIEWS.

$\mathbf{B Y}$

\section{SIR HENRY HOLLAND, BART., M.D.}

F.R.S., D.C.L. Oxon., \&c. \&c. FELLOW OP THE ROYAL COLLEGE OF PHYSICIANS: PHISICIAN IN ORDINAKY TO THE QUEEN.

NEW EDITION.

LONDON:

LONGMAN, GREEN, LONGMAN, ROBERTS, \& GREEN. 1862. 



\section{H 675 \\ 1862}

\section{My dear Milman,}

Had our mutual friend Lord Macaulay been yet alive, I should probably have sought to inscribe to him the volume I am now publishing. Will you permit me to ask you, as one of those to whom he was most warmly attached, to give it, in his stead, the sanction of your name; and to accept this Dedication as some slight memorial of our long friendship, as well as of my admiration of those various writings with which you have enriched English literature.

Ever yours affectionately,

H. Holland.

The Very Rev. the Dean of St. Paul's. 



\section{PREFACE TO SECOND EDITION.}

NOTHER edition of these Essays being called for, A I have carefully revised the whole volume, and particularly the scientific articles; making such additions as seemed desirable for the more complete elucidation of the subjects under review. It is a striking proof of the rapid advancement of physical science, that even in the short interval of two months since the first edition was published, discoveries have been made known which no scientific record could rightly omit to notice. The addition of a few pages has enabled me, either in the text or notes, to advert to the more remarkable of these discoveries. I should gladly have dwelt much longer on them (as upon many other instances of the progress of physical knowledge), had not the original plan of these articles restricted me to those more general views, of which I have spoken in the preface to the former edition. To this preface I may perhaps be allowed to refer the reader, in explanation of the circumstances under which they were written, and the objects they were intended to fulfill.

July 15, 1862. 



\section{PREFACE TO FIRST EDITION.}

THE Essays collected in this volume were written in 1 great part during journeys or voyages which I have been accustomed to make in the autumn of every year; - under the conviction, justified by experience, that in no other way could I equally maintain health amid the labours of a London professional life. During the last twenty years, aided by facilities unknown before, I have repeatedly visited America, and the nearer parts of Africa and Asia ; - hastily, I must admit, yet never without some good attained. Travelling frequently alone, however, and often by routes rarely followed, it would have been difficult for me to sustain the solitude of particular times and places in such journeys, without some personal occupation constantly within reach. This occupation I have found in writing the several papers now republished, or such principal portions of them as formed a framework, to be filled up on my return home. The employment so gained was as agreeable as beneficial; - perhaps not less so, from the contrast it often afforded to the scenes and objects around me, at the time when I had recourse to it.

The greater number of the Essays, thus composed, 
will be seen to have relation to the progress and actual state of the physical sciences. Written with the object of such illustration, they will, I trust, be read under the same view; - that is, not as formal treatises on these several subjects, but as general outlines, denoting the new methods and spirit of modern research; the more recent and remarkable discoveries in each science ; and those mutual connections of all, the knowledge of which is among the highest attainments of man in his study of the natural world around him.

In these Articles, more purely scientific, many alterations have been required; - partly from the rapid growth of physical knowledge since they were first published;-partly from the collected form in which they now appear. Various additions have been made, either in the text or by notes, to remedy deficiencies from the former cause; - many omissions also, to obviate the repetitions which could scarcely be avoided in papers written at different times, and for different Reviews. To meet the same requirements, I have further, in several cases, transferred portions of one article to another ; or even taken parts from essays not included in this volume. A few anachronisms will probably still be found; but none such, I trust, as can create error or embarrassment to the reader. Certain repetitions also may be noticed (as with regard to the influence of the Atomic doctrine on Modern Chemistry); but these will be seen to depend on the particular object of the first Essay, which, treating of physical science at large, necessarily took its illustrations from the several branches of science composing this great whole. 
The topics actually discussed in these papers are so various, that it has not seemed needful to place them in the order of their original publication. Nor have I attempted other arrangement than that of bringing together, in one or two cases, those which have some slight affinity of subject.

I have already avowed a certain feeling of personal interest in many of these Essays, from their association with various scenes and incidents of travel, and with some localities which belong to the history of the world. This interest I cannot expect to convey to others, and the papers must therefore rest on whatever value they may themselves happen to possess. It would be false modesty not to mention that I have been asked to publish them thus together, by several persons whose opinion I have every reason to respect. To one of these - a dear friend, and, in later years; a family connection - I should probably have ventured, had he happily still been living, to dedicate this volume. Yet I might well have hesitated to do so, from the fear of provoking a comparison with those admirable Essays he himself contributed to the 'Edinburgh Review ;' the volumes containing which form one of the precious bequests he has made to the language and literature of his country. 



\section{CONTENTS.}

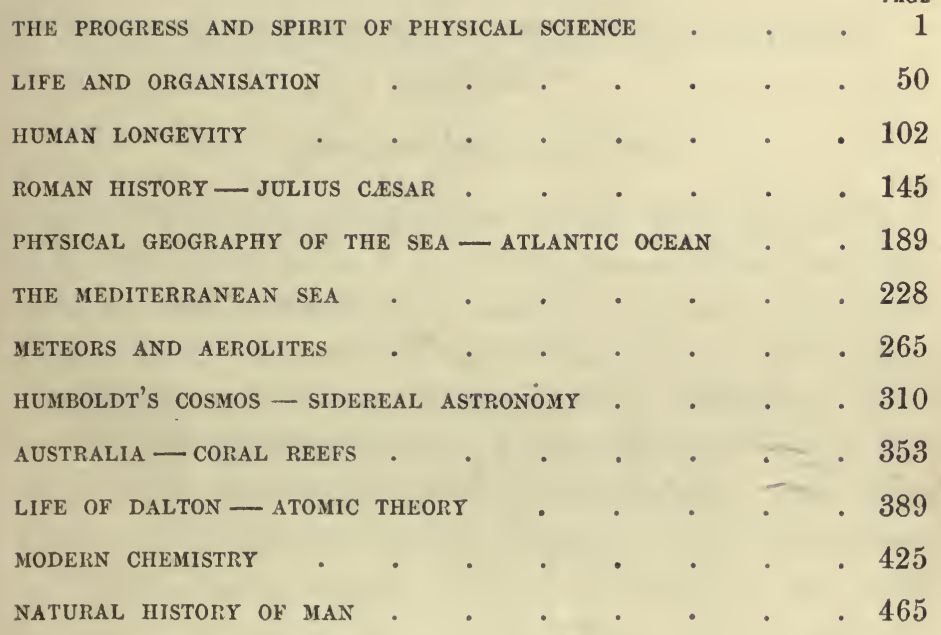





\section{ESSAYS}

oN

\section{SCIENTIFIC AND OTHER SUBJECTS.}

\section{THE PROGRESS AND SPIRIT OF PHYSICAL SCIENCE.*}

[EDINBURGh Reytew, JULY 1858.]

A LMOST every age of human history has either given to whether truly or fancifully, its distinctive place in the records of the world. It would be easy to find and to apply many such epithets to the remarkable period in which our own lot is cast; abounding, as it does, in characteristics which distinguish it from any that have ever gone before. One, which we cannot doubt that our own posterity will adopt, inasmuch as it affirms a fact equally obvious and certain, is, that we are living in an age of transition; - a period when changes, deeply and permanently affecting the whole condition of

* 1. Essays on the Spirit of the Inductive Philosophy, the Unity of Worlds, and the Philosophy of Creation. By the Rev. Baden Powell, M.A., F.R.S., \&c., Savilian Professor of Geometry in the University of Oxford. London, 1855.

2. The Co-relation of Physical Forces. By W. R. Grove, Q.C. M.A, F.R.S., \&c. Third Edition. London, 1855.

3. On the Conservation of Force. By Professor Faraday, D.C.L., F.R.S., \&c. \&c.

4. Essays from the Edinburgh and Quarterly Reviews. By Sir John F. W. Herschel, Bart. K.H. London, 1857.

5. Nomos: an Attempt to demonstrate a Central Physical Law in Nature. London, 1856. 
mankind, are occurring more rapidly, as well as extensively, than at any prior time in human history. The fact is one which lies on the very surface of all that we see in the world around us. No man of common understanding, even in the narrowest circle of observation, but must mark the continual shifting of things before him; reversing, in many cases, the maxims and usages which are the inheritance of centuries, and altering in a thousand ways the present conditions of material and social life. The philosopher who looks from a higher level, and upon a more distant horizon, discerns in these changes a wider and more lasting influence. He sees that they involve the relations of races and communities of men over the whole face of the globe; and that they are destined, sooner or later, to obliterate many of those diversities and lines of demarcation, which, however originally produced, seemed almost to dissever the species, in the contrast of human existence they afford. He takes further note of what is the great agent in this and other changes, that wonderful progress in physical philosophy, which has placed new powers in the hands of man - powers transcending in their strangeness and grandeur the wildest fables and dreams of antiquity, and the effects of which are already felt in every part of the habitable earth. He sees the march of discovery continually going on; new paths opened; new instruments and methods of research brought into action; and new laws evolved, giving connection and combination to the facts and phenomena which unceasingly accumulate around us.

Closely, or even necessarily, connected with the changes last denoted, is the topic to which, as suggested by the works before us, we would especially invite the attention of our readers. We allude to the concurrent changes taking place in the spirit and scope of physical philosophy at large; scarcely less remarkable in their nature and influence than 
the discoveries in which they originate, and by which they are sanctioned. Modern science, in its dealings with the great physical powers or elementary forces which pervade the material world, has been led, or even forced, into a bolder form and method of enquiry. Inductions of a higher class have been reached, and generalisations attained, going far beyond those subordinate laws in which science was formerly satisfied to rest. Experiment and observation, as the agents in acquiring knowledge, must always to a certain extent be alike in their objects and methods of pursuit. But the precision and refinements of modern experiment - partly due to greater perfection of instruments, partly to the higher principles on which enquiry is based - strikingly distinguish it from that of any anterior time. With every allowance for illustrious exceptions, it is impossible to make the comparison, and not to see that the physical researches of our own day have a larger scope and more connected aim than heretofore; - that experiment is no longer tentative merely, but suggested by views which stretch beyond the immediate result, and ever hold in sight those general laws which work in the universe at large. Nor is any power so gained permitted to be dormant or inert. If thought suggests experiment, experiment ministers fresh materials to thought; and the philosopher working boldly with the new forces at his command, and under the guidance of hypotheses which extend to the very confines of human intelligence, obtains results which almost startle the imagination by the inroads they seem to make on the mysteries beyond. When flying along the railroad at forty or fifty miles an hour, with a slender wire beside us, conveying, with speed scarcely measurable, the news of nations, the demands of commerce, or the fates of war, we have an example (though few estimate it fully) of those mighty attainments which bind to do our bidding, elements before unknown or uncontrolled by man, 
and which give certainty of other and similar attainments in time yet to come.

Admitting that hypothesis, and this often of adventurous kind - the 'animi jactus liber' - blends itself largely with the recent progress of physical science, we would in no way impugn this powerful instrument and aid of research; the use of which, under due limitation, is justified equally by reason and experience. In all enquiries of this nature, except those strictly mathematical, certainty and conjecture necessarily and closely commingle. The speculation or bare analogy of one day becomes the scientific induction of the next; and even where hypothesis is not thus happily fated, it still has often high value as an interpreter and provisional guide to the truths sought for. All sciences, and very especially those of optics, chemistry, and electricity, furnish notable instances to this effect; and have rescued hypothesis, in the philosophical sense of the term, from the reproach which it was once the fashion to cast upon it. Such vindication, however, affords no sanction to that spirit which pushes mere speculation far in advance of experiment and observation, and adventures rashly into fields not prepared for human culture, if indeed ever. accessible to it. Eccentric theories of this kind, the produce of imperfect knowledge or illogical understanding, will ever be found in the path of science; perplexing, it may be, to those who loosely follow it; but disappearing one after another, as truth pursues its steady course amidst them. The mysteries of organic life, approached with caution by the true philosopher, are an especial seduction to these framers of new systems; - systems which it becomes easy to coin, under shelter of a vague phraseology, and aided by the very obscurity of the subject with which they deal.

While speaking thus generally on the spirit and methods of modern science, we may notice the fact, that there is 
scarcely one of the legitimate hypotheses of our own time, or even any great law founded on the soundest inductions from experiment, which is not prefigured in some way, more or less distinctly, in the philosophy of former ages. We might, had we space for it, give many curious instances of these anticipations; and assign reasons why they should especially be found in the more recondite parts of philosophy, such as the origin of matter, the qualities and combinations of atoms, the theories of space, ether, forces, \&c., - transcendental questions which press themselves upon the thought of the metaphysician, as well as of the naturalist and mathematician, in contemplating the phenomena of the universe. The astronomy of the Greeks indeed, even unaided by the telescope, went beyond all other science of the time; and the great names of Aristotle and Archimedes stand out in antiquity as men who knew the value of exact observation and experiment in the study of nature. But it was chiefly through the avenues of thought and speculation, little aided by experiment or systematic research, that the subtlety of a few rare spirits in each early. age came upon the traces of physical truths, which modern science has approached by more certain roads, and made the lawful prize of inductive research. What then were but hasty and transient glances into these profound parts of philosophy have now become a steady insight into the great physical laws under which are embodied all the phenomena of the natural world.

We have placed at the head of this article the titles of several recent works, well fitted, by their various merits and by the eminence of their authors, to illustrate the view we have briefly given of the present aspects of physical philosophy, as well as to indicate those future prospects of science, whch may fairly be inferred from the spirit in which it is now pursued. We might largely multiply the number, were 
we to include even a small proportion of the systematic or elementary works; - the lectures, memoirs, and addresses to scientific bodies - or the articles in periodicals, which, under the influence of this new vigour of enquiry, and the practical popularity of many of its topics, have opened their pages to meet the demand for more familiar information than scientific treatises can afford. These topics, in fact, include not only the sciences treating of the simpler inorganic conditions of matter, and the elementary forces (heat, light, electricity, gravitation, and chemical affinity) which act upon the material world; - but also animal and vegetable physiology in their whole extent, and those wonderful laws of organic life, connecting matter with vitality, instincts and intellect, under the numberless forms and species which are placed before us for our contemplation. In surveying this vast field of natural knowledge for the purposes just indicated, we must of necessity limit ourselves to a broad outline; thereby forfeiting in some part the interest which belongs to the familiar illustrations of each particular science, but gaining in compensation a more comprehensive view of the relation between the different sciences and of those great discoveries in all which are ever tending to bring them into closer connection and subjection to common laws. We need scarcely dwell on the importance of such general views, and their influence on the spirit and progress of physical philosophy. We shall have occasion immediately to illustrate it, in speaking of the efforts made by some of the most eminent men of science of our day to give concentration and unity to classes of phenomena hitherto regarded as having no co-relation or common principle of action.

We do not undertake to analyse in detail, or even to notice, all the works before us. To one of them, however, the Essays of Professor Baden Powell, we must refer, as expounding more distinctly than any other, that present spirit 
and scope of physical science to which we have just alluded. In the first of these Essays, Mr. Powell describes the doctrines he adrocates under the titles of 'Unity of Sciences,' and 'Uniformity of Nature,' - terms meant to express, but expressing too strongly, those admirable generalisations which have connected under common laws phenomena seemingly the most remote and unlike, and are continually tending still further so to connect them. Taking the subject in this general sense, we cannot hesitate to regard it as one of the very highest which can be submitted to the human understanding. The unfulfilled objects of science, as well as its ultimate end and aim, evidently lie in this direction; and none can be indifferent to the wonderful results which every year is disclosing to researches pursued on this principle. Among those who have laboured most successfully for this especial object are the eminent men whose discoveries in particular branches of science have given them merited fame in the world. If out of many contemporaries we were to select a few who have done most to elevate physical science by generalisation of its phenomena and laws, the names of Arago, Faraday, Herschel, and Humboldt occur at once as first in this career. These philosophers have looked upon the world of nature in its largest aspects, and made their several discoveries subservient to this great object; thereby widening the circle of facts and phenomena, and at the same time drawing them more closely towards that centre in which we find so many sciences to converge.

Nevertheless we must not allow these terms of ' Unity of Science,' ' Unity of Principle,' and ' Unity of Law,' to usurp too much on the understanding. Professor Powell gives undue force to such phrases; which, strictly examined, have no reality in our actual knowledge. It is true that there is various high authority for their use, as for that of language analogous in effect. Humboldt, in several passages of his 
' Cosmos ' - and, at an earlier period, D'Alembert and Laplace, - have sanctioned the general conception, though not really defining it beyond that attempt at generalisation just noticed ; and which would have existed even if no such mysterious word as 'Unity' had been used to signify the ultimate end in view. We readily admit it as probable or certain, that numerous facts hitherto insulated or anomalous, and even whole classes of phenomena unexplained by science, will hereafter be submitted to common and known laws. And we further believe that many laws themselves, now of partial application, will hereafter merge in others of higher scope and generality. We shall speedily have to notice certain cases where this amalgamation has so far advanced as to furnish an entirely new basis for research, scarcely seen or anticipated before. But admitting what we have full right to presume, that this concentration may yet be carried much further, still the attainment or even the conception of unity, in any strict sense of the word, lies indefinitely beyond; shrouded by an obscurity which words may seek to penetrate, but which human intellect can reach only in that one sublime sense of the unity of the Divine Creating Power. We may reduce to a small number the many forms of matter which are elementary to our present knowledge; - we may show the identity of certain forces, hitherto deemed elementary, by their mutual convertibility; - we may accept the phrase of Laplace, 'Les phénomènes de la Nature ne sont que les résultats mathématiques d'un petit nombre de lois immuables;' and yet we shall never prove that there is but one kind of matter or one nature of force, or that a single law governs all the phenomena around us. To put forward, therefore, the phrase and conception of 'Unity of Science' as the final term of our labours, is to inflict a metaphysical issue upon them, for which there is no warranty either in reason or practical use. Bishop Berkeley has somewhere 
spoken of ultimate ratios in mathematics as the 'ghosts of departed quantities.' With like reason we might call the unity of some of our recent philosophers the 'ghost of departed pluralities;' having this quality of ghosthood, moreover, that there is nothing truly tangible or substantial about it.

We have dwelt thus much on these preliminary topics because, while they indicate what may be considered the exaggerations and excesses of theory, they show at the same time that spirit and propensity of modern science of which we have before spoken; and which, duly regarded, has been the source of all its high attainments. We now proceed to such details as may best illustrate this spirit in its application to different branches of science; selecting, amidst the multitude of examples, those especially which involve either some new physical principle or some new method of physical enquiry. It has been said by one who could well estimate the value of the latter, 'La connoissance de la méthode qui a guidé l'homme de génie, n'est pas moins utile au progrés de la science que ses découvertes.' A new method is often indeed in itself the greatest discovery, and betokening the highest genius in him to whom it is due.

In dealing with this wide subject, the first and most material division is that between the Forces acting on or in Matter ; and the various forms of Matter ; inorganic or organic, so acted upon. With full admission of the difficulty of defining the abstract nature of matter and force, and their mutual relations in the universe, this distinction is still the only one which our intelligence can apprehend or practically apply to the objective phenomena ever present and active around us.

In regard to matter and force it may undoubtedly be affirmed that all questions as to their nature become more 
difficult and abstruse in proportion as we generalise and reduce them to their simplest terms. With respect to force, more especially the most eminent philosophers of our time, while declining any metaphysical definition, have been constrained to adopt new methods of regarding and describing it, in those various actions upon or through matter which testify to its presence and energy. Centres of force (an expression due to Boscovich in its scientific use), lines of force, polar force, \&c., are terms found necessary to express the several modes of force in action, irrespectively of all questions as to its abstract nature, or especial relations to matter. Under the gradual adoption of this new language, there has been a corresponding abandonment of phrases, more hypothetical in themselves, and far less fitted to aid the progress of scientific enquiry. As such we may denote that expression, current even in some of our best systematic works, of the ' imponderable substances or forms of matter ;' which, as describing heat, light, and electricity, makes assumptions wholly unproved; and in excluding gravitation, chemical, mechanical and vital forces from the same category, affirms a distinction which we do not absolutely know to exist. For the notion of an imponderable element (if notion it can be called) that of a mode of change, or motion of matter, might probably in all these cases be more truly as well as advantageously substituted. Such phrase better defines the present limit of our knowledge, and marks a possible path of progress beyond. Science, it may fairly be said, is. constantly tending to a closer form of logic in these matters; and simple induction from facts, unfettered by names and prior notions, is here as elsewhere the best guide to all ulterior discovery.

The great problem respecting force in the most general conception of it as a motive power on matter, is involved in the question, whether it can ever really be lost or extinguished, or even lessened? or whether the;seeming cessation 
and limits to its action are not merely conversions or translations of power, testified in other forms and effects of material change? Most persons, justified by outward appearances, would answer at once that a force has ceased to exist, when the motions or other effects it induces on matter are no longer. present. The question, however, is one which rises far above the mere evidence of the senses. Vaguely suggested at former periods, it has been adopted in a definite shape by the philosophers of our own time; forced upon them, we may say, by the course and character of recent discovery. It is the question which forms the main topic of Mr. Faraday's lecture, which lies before us, on the 'Conservation of Force;' and we willingly quote a few lines, both from the intrinsic weight of all that comes from this source and as expressing what we consider to be the growing conviction of all who have grappled with this great problem of modern science.

To admit that force may be destructible or can altogether disappear, would be to admit that matter could be uncreated, for we know matter only by its forces. Agreeing with those who admit the conservation of force to be a principle in physics as large and sure as that of the indestructibility of matter, or the invariability of gravity, I think that no particular idea of force has a right to unlimited or unqualified acceptance, that does not include assent to it. . . . Therefore I urge, that the conservation of force ought to be admitted as a physical principle in all our hypotheses, whether partial or general, regarding the actions of matter.

This question, as we have said, was forced upon the attention of men of science by the very nature of their recent researches, and especially by the doctrine based upon them, which is now developing itself under the title of the ' $\mathrm{Co}$ relation of Physical Forces;' a description modest as well as apposite of a theory, which if matured, as we think it certain to be, into full truth, will give new foundation and guidance to the whole course of physical enquiry. In the work of Mr. Grove, bearing this title, and prefixed to our article, we have 
the first and most able exposition of the doctrine. Partial suggestions of it, both in England and Germany, had already occurred; but we owe to Mr. Grove its distinct enunciation as a physical principle, and the illustration of this principle by instances drawn from his own researches and those of others, which give it all the characters of a new physical law. His work, of which the third edition is before us, is remarkable, not only for the bold and exact logic of its inductions, but also for its clearness and simplicity of style; qualities valuable in all scientific writings, and essential on subjects like those here treated of.

By the term co-relation, as applied to physical forces, Mr. Grove means to convey the general idea of reciprocal production; - that is, that any force capable of producing another, may reciprocally be produced by it. But the principle here involved, as well as its wide scope, will be better understood by taking co-relation to express generally those relations of forces which render them mutually and constantly convertible - one form or manifestation of force generating another, so as to bring together into the same series of effects, physical actions and changes seemingly the most remote and dissimilar. Thus, to take a familiar but striking instance. The same single electrical current from a voltaic battery is capable in its circuit of evolving heat and light - of creating magnets - of producing mechanical force - of violently affecting the nervous and muscular organisation - and of inducing, by decomposition or combination, the most powerful chemical changes - simply according to the nature of the different material objects which the experimentalist interposes in the circuit, so as to subject them to this current of power. Here then (gravitation excepted) we find all the great natural forces, of which we have present knowledge, evolved from a single source; and that source, be it remarked, a chemical change of affinities, giving origin 
to the electrical current, and thereby affording fresh proof of the reciprocity of actions alluded to above. One form of force always disappears as another is evolved.

We might give, had we space for them, many other curious instances of this reciprocity of relation, as manifested by the several forces of heat, electricity, magnetism, mechanical power, and chemical affinity. One we may select, as an example of beautiful contrivance as well as striking results. By a certain combination of apparatus, in which light, acting through the daguerreotype, was the initiating force, Mr. Grove obtained first the chemical action upon the plate; thence a current of electricity circulating through wires; next magnetism by a coil of these wires; then the production of heat, testified by the delicate helix of Breguet; and finally, motion, as shown by the needles of the galvanometer. Instances of this kind have rapidly multiplied, since the corelation and convertibility of forces has been recognised as a principle and applied to research. They are derived not solely from recent experiment, but even more frequently and fruitfully from phenomena already familiar to us as facts, but which awaited for their illustration the happy induction now attained.

The beauty of this principle, however, is not limited to the expression of the reciprocity or mutual convertibility of the physical forces with which we are dealing. There is every reason to believe in a further co-relation as regards their equivalents of power or measurable quantitative effects. Though this generalisation is still not complete, numerous cases occur where it is thoroughly attested by the results of experiment. The discoveries of Faraday have furnished some of the most striking examples of constant quantitative relation between electrical power and chemical actions and changes. The researches of Dulong, Petit, and Neumann, show very remarkable relations between chemical affinity and heat, 
in proving that the specific heats of certain substances, compound as well as simple, when multiplied by their chemical equivalents, give a constant quantity as the product. And again, the experiments recently made by Mr. Joule and Professor W. Thomson, on the mutual convertibility of heat and dynamical force, demonstrate the remarkable fact that, in whatever way mechanical force is employed to produce heat, the same amount of heat is produced by the same amount of force. We doubt not that the progress of science will so multiply the number of these instances of quantitative relation and equivalents of power, as ultimately to submit them to some general law, as well as to practical application in various forms. The very phrases, if duly weighed, show that approach to mathematical proof, which is the surest test of truth. Where we can bring numbers to verify results obtained, and to predict results sought for, we may be certain that we are treading on safe ground.

It will be noticed that we have not hitherto spoken of Gravitation as a physical force; though it is the one with which we are most familiar in every incident of life, and to which we look as the most universal agent upon matter, as well on the globe we inhabit, as in the innumerable worlds surrounding us in space. We place it apart from other physical forces, because, while thus familiar to our senses in its effects, it is to our deeper meditation the most mysterious as well as vast and sublime of the powers which act in the universe. Human genius has discovered and mathematically defined its laws. By knowledge of these laws, human science has been carried, and is ever penetrating farther, beyond our own planetary system; while within this system, they have enabled us to predict events in time and space, and to define physical conditions of the planets and their satellites, seemingly unapproachable by man. With all this knowledge and perpetual application of the power, 
of its nature and essence we are utterly ignorant. Science has dealt with its effects only, without really approaching a step nearer to the cause, than when Newton declared that he must leave to the consideration of his readers the question whether the agent producing gravity was material or not. Hypotheses have grown up - such as that of gravific atoms permeating all space, of Le Sage; or the residual force theory of Mosotti, connecting gravity with molecular attractions - but none which satisfy fully the exigencies of the case. The research is even made more difficult by the simplicity and invariability of the power in question. It evolves in certain cases, and constantly controls or modifies, the other forces acting on matter; but has no such relations to them as they have to one another; - no reciprocal production or mutual convertibility; nor the duality of action belonging peculiarly to the electrical and magnetic forces; nor lines of propagation and polarisation, such as we recognise in light and heat; nor those molecular changes manifested in acts of chemical affinity. Whether any - or if any, through what new avenues - closer approach may hereafter be made to the solution of this great problem of gravity, bringing it into closer connection with the other great forces of nature, we cannot here enquire. But in speaking of the forces which act upon matter, it was impossible to omit this the most universal of all - innate and incorporate we might almost say, in matter itself.

Nor can we rightly avoid in this place some allusion to the equally abstruse subject (though rendered so by very different causes) of the mutual relations of the physical and vital forces - a topic handled with great ability by Dr. Carpenter, in a paper in the 'Philosophical Transactions,' a few years ago, and more recently in the systematic works of this physiologist. Without going into the depths of this question, we may say that the tendency of all recent research has been to 
impugn the doctrine of vitality, both in animal and vegetable life, as a distinct force or power; and to resolve its alleged functions, whether of organisation, maintenance, or reproduction, into those same physical forces which act on the inorganic matter of the world around us. That this is true to a certain extent cannot indeed be doubted. That heat and light, and more especially the former, are intimately concerned in all the phenomena of vital organisation, is a fact familiar to us from a thousand examples. The researches of Liebig and others have shown how very closely chemical processes are engaged even under the strict law of definite proportions - in all the great processes belonging to animal life, assimilation, secretion, respiration, animal heat, \&c.; while the discoveries of Matteucci and Du Bois Raymond have demonstrated the curious and exquisitely subtle relations which exist between electricity and the nervous and muscular functions; not indeed proving the identity of electricity with the nervous element of force, but countenancing this view beyond all prior expectation.

In thus discussing the relation of the physical and vital forces as applied especially to man, we continually approach that line, hard indeed to discriminate or define, which separates the mere vital or automatic acts from the proper functions of mind, consciousness, thought, feeling, and volition. On this debatable land we encounter at once the old questions, so long the subject of philosophical speculation, and destined, as far as we can see, ever so to remain. Human science on this point is as feeble as it was two thousand years ago, and beset by exactly the same difficulties. We have just been speaking of forces which are co-related and measurable in their effects. We come here to powers and functions wholly incommensurable either with material qualities or physical forces; yet so linked with both under the present conditions of existence, that not even personal consciousness, 
on certain points the surest of all teachers, can mark any clear boundary line. Those who have sought to decipher or define these proximate relations of matter and mind have but substituted barren words for the realities of knowledge. Mr. Baden Powell himself, while stretching the domain of physical causes to the total phenomena of animal life, yet finds a limit here; and somewhat abruptly closes his argument by observing that the assertion of a moral and spiritual nature in man refers essentially to ' $a$ different order of things, apart from and transcending any material ideas whatsoever.' To some such conclusion, however expressed, all must come who honestly and reasonably approach this question.

We have dwelt thus long on the subject of the physical forces - the 'imponderables' of former systems - as illustrating at once a great doctrine of modern science, and the general spirit of philosophy at the present time. We are far, however, from having exhausted the subject. Questions crowd round and converge upon it from every side; some of these so subtle in kind that we might well call them metaphysical, but for the caution ever needful in admitting this term into the domain of science. Such are, to state briefly a few of them, the question whether forces can exist, except in connection with matter, and manifested by its changes? whether what we call forces may, intelligibly and consistently with phenomena, be regarded simply as molecular actions, or modes of motion in matter? - whether (to revert to a question urged before) they can ever by possibility be annulled or even rendered latent? - whether, in admitting this constant combination and convertibility of forces, we do not virtually admit a constant amount of force, variously manifested, to be always present in the universe? - and whether, in such case, we can ever rightly speak of an initial force, otherwise than in the sense of those acts of 
creation in which all human knowledge ends? These and other like questions belong to the philosophy of our day; some of them shadowed out in the hypotheses of antiquity; now approached through the safer avenues of experiment and sound induction. How far these may lead us to the future more complete solution of the problems suggested we cannot here stop to enquire.

In passing from the province of Forces acting on matter, to that of Matter thus acted on, we have yet to traverse another debateable ground, on which science is seeking to find some firm footing, as well in explanation of known phenomena as for purposes of further research. We allude here to the question regarding the physical condition of space itself; of those inter-planetary and inter-sidereal distances, some of them hardly measurable by numbers, and such, in truth, as no effort of mind can compass or conceive. Are we to regard this vastness of space as void of matter - a mere vacuum, through which the numberless worlds we see as stars or planets are dispersed? Or may we better contemplate it as pervaded throughout by some material medium, though so rare and attenuated, that no form of matter of which our senses are cognisant can rightly interpret it to our reason? The question must no longer be argued in that mystical language of ' nature abhorring a vacuum,' which satisfied the demands of an earlier philosophy. Nor can we evade it by the adoption of terms such as ether, ethereal medium, \&c., which, though sanctioned by some great names, go little further than to shelter a vague and incomplete solution. Modern science seeks urgently for proof that matter, in some condition, does exist throughout space; and in such continuity, however rare it be, that forces may be transmitted by or through the medium thus afforded. Two great powers, gravitation and light, undoubtedly reach us from the most 
remote regions of space. There is presumption, approaching to certainty, that heat is associated with light in its origin, as a concomitant, if not convertible force. More doubt exists as to the transmission through space of the electric or magnetic powers; but numerous observations tend to justify this belief, and such facts, as we shall see hereafter, are every day multiplying around us.

How then are these forces, or any of them, transmitted to and fro in the universe? If we say that the tides of the ocean are raised, or the perturbations of a planet produced, without any intervening medium between the bodies affected and those affecting them, we quit the domain of physics altogether, and put an abrupt end to enquiry. Newton has expressed himself strongly on this matter, in saying, 'To suppose that one body may act upon another at a distance, through a vacuum, without the mediation of anything else, by and through which their action and force may be conveyed from one to another, is to me so great an absurdity that I believe no man who has in philosophical matters a competent faculty of thinking, can ever fall into it.' The conviction which his conception of gravity thus impressed on Newton's mind, is enforced upon us not less cogently by the undulatory theory of light. This theory - based on mathematical proof, and capable not merely of explaining phenomena before known, but of predicting others evolved by later research presumes of necessity the existence of an elastic medium, whatever its nature, through which these undulations are transmitted. We say of necessity, because it is logically thus to our reason. Not solely on the analogy of air and other elastic media, but as the only conception we can form to the mind of undulation singly considered, the presence of a medium is essential to its existence and effects. And this fully recognised, the inferences become of magnificent kind. The progressive retardation of Encke's comet, the aspects 
of the zodiacal light, and the numberless meteorites traversing the sky, afford presumption of such material media everywhere existing within our own solar system; but the argument we have just stated carries us far beyond this limit, to the most remote parts of that sidereal and nebular space from which light reaches the eye of man. We might bring the phenomena of heat into evidence on the same point; though less strikingly and conclusively than those of gravitation and light, of which we have just spoken.

In coming finally to those several sciences which deal with Matter in its more recognised forms, we must once again repeat that our object is simply that of indicating the spirit and scope of modern science, as illustrated by its new objects and methods, and by the high attainments at which it has arrived. Volumes would be needed to give even an approximate idea of the particular discoveries, whether from experiment or observation, which have conduced to these results. In the hasty view we are taking, we can but notice such as are most striking in character. Nor are we called upon to do this methodically; since, as already mentioned, one of the most eminent successes of our time is that of having brought all the branches of physical science into closer connection and subordination to more general laws; and in illustrating these new connections, examples crowd upon us from sources seemingly the most remote.

Humboldt, in his Cosmos, has rightly given to Astronomy'the science of the universe without' - the first place in his picture of physical knowledge. So much has lately been written on this science - the highest glory, it may well be deemed, of the human intellect - that we need only allude to a few of its more recent attainments; not surpassing indeed those discoveries which we owe to the genius of a prior time, yet so extending the doctrine of universal gravi- 
tation in the variety and refinement of its applications, that new grandeur is given to this great law of nature. We may take one or two examples, among many that offer themselves, from our own planetary system; where this power is more within our cognisance, both in its simple effects and in those complex perturbations of orbits, which have taxed, but not overcome, the efforts of mathematicians. The first instance - one of those familiar to the world for the moment, but speedily forgotten - is a discovery made by means of these very perturbations. The movements of Uranus, then (1846) supposed the most remote planet of our system, were found to be disturbed by some external influence not referrible to causes within its orbit, as could be shown; but due to some material attraction from without. Another planet alone could answer these conditions. Science set itself to work in the persons of two eminent mathematicians, Adams and Leverrier; - the position of the disturbing body was determined by them simultaneously, but independently; - telescopes followed their guidance, and Neptune was added to the number of our planets. The method of discovery here has higher interest than the fact itself; though now but one of numerous instances in science, where results can be predicted with hardly less certainty than if attained and present to the senses.

A second example we may cite, in proof of the exactness, or even delicate minuteness, with which modern astronomy pursues the vast objects of its science. The complex irregularities of the Moon's motions have long put to test all the resources of analysis, and are scarcely even yet fully submitted to our knowledge. Chiefly, of course, they depend on the relative position and distances of the sun and earth; and Laplace had shown, not only the secular acceleration of mean motion, produced by the increasing eccentricity of the earth's orbit, but also a small irregularity depending on the 
spheroidal figure of the earth itself. His suggestion that the oblateness of the earth's spheroid might reciprocally be determined by this irregularity of the moon's motion led Burg to a calculation, the results of which closely tallied with the best measurements and pendulum observations. Very recently new and more delicate causes of lunar disturbance have been indicated, as depending on the action of the planet Venus;-first, indirectly, by its perturbing the motion of the earth, altering our distance from the sun, and thereby affecting the motion and position of the moon during periods of 120 years; - secondly, by a minute disturbance arising from the direct action of Venus on the moon itself. In all these. cases the theory accords with the phenomena observed; and this accordance well illustrates the perfection of use which the great law of gravitation has now attained.

In passing the bounds of our own system - narrow, we may call them, in relation to what lies beyond - we lose in great part the guidance of this law; though retaining such proof of its equal and probably similar operation in the most distant regions of space, as almost to force upon us the conclusion (warranted indeed by other considerations) that Motion is universal and constant in all matter - that nothing in the universe around us is at absolute rest. To prove the continuous movement of the solar system in space, with the direction and rate of its motion; - to confirm this wonderful fact by the discovery of the proper and absolute motions of other stars; - to determine by parallactic observations of incredible delicacy the distances of certain of the fixed stars, and to measure these distances by the years which light takes to traverse them;-to demonstrate, among the many thousand double or multiple stars now discovered, those orbits and periods of revolution which obey the same law that brought Newton's apple to the ground;-to gauge by refined processes our own nebula of the Milky Way; - to discover 
and assign the place of more than 3,000 other nebulæ, resolving many of them into systems of stars, and by admirable methods obtaining some approximate idea of their distances; - these have been among the undertakings of modern sidereal astronomy; admirably fulfilled by the eminent men who have devoted themselves to this science, the two Herschels, Struve, Bessel, Airy, Argelander, Peters, \&c. Sublime even in their simplest enunciation, these problems will be seen to involve results as to space and time which border on infinity; and as such illustrate well those arduous aspirations of modern science which it is our present object to indicate.

Though not easy in a science like this to set limits to its future scope, yet is it difficult to suppose any ulterior discovery which can do more than aid in filling up this vast outline. If new laws are discoverable in our own system, we may perhaps presume them to relate to the rotation of the planets on their axes, and to their respective densities ; - an important series of facts arbitrary to our present knowledge, but doubtless due to determinate physical causes, and therefore open to physical research. It is possible, seeing the distances some comets reach in their aphelia, that another planet may exist even beyond Neptune; which discovery, if ever made, would be so through the observed perturbations of Neptune itself. Certain irregularities in the orbital motions of Mercury have led Leverrier to suspect the existence of an inferior planet, or masses of matter, still closer to the sun; but no actual observation has yet been made which can be admitted as verifying this idea.

In the Sidereal system, of which our solar system forms a single part, much yet remains for future completion. Nothing is more wonderful than the phenomena, periodical or otherwise, of the variable stars, which are now largely catalogued in our books. Ages may be required to gather any certain 
induction from our observations upon them. But ages are the field in which the astronomer works; and each present fact, duly recorded, ministers to the higher knowledge, which is the harvest of the future. The research into the proper motions of the stars, already noticed, is sure to be greatly extended, and may possibly connect itself in the end (as Mädler has already sought to connect it) with the discovery of some centre of attraction and movement to the whole sidereal system. If such central body or point in space were ever ascertained, it would still be simply an expression of the law of universal gravitation; but how sublime an expression, and how wonderful as a result of the genius and labours of man!

But the limit does not lie even here. The telescope of the astronomer, enlarged in its powers and more perfect in all its appliances, is continually engaged among those other sidereal or nebular systems, the remoteness of which goes far to express all that man can ever conceive of the infinite in space. Whoever has inspected the admirable portraits of nebulæ, as seen through Lord Rosse's great reflector, will comprehend in part the magnitude of this research, and of the problems it puts before us. The aspects and multiplicity of the spiral nebulæ, though hardly sanctioning the notion of any new law of matter, yet well warrant the belief in some common but unknown cause conducing to this singular effect. A matter of still higher interest is suggested to us in the question, whether there exist in these nebulous lights, or elsewhere in space, matter not yet condensed or shapen into forms - the material, it may be, of future worlds, and in different stages of progressive concentration, but still not aggregated as such. The resolution into clusters of stars, by high telescopic power, of many nebulæ before thought irresolvable, alters the degree of presumption, but does not settle the question. The comparison of different nebulæ, as they now exist, and of their several relations to centres or points of greatest condensation, 
would seem the sole probable avenue to further knowledge; since any changes in the figure, condensation, luminousness, or other aspects of these nebular systems must, upon every analogy of the more proximate parts of the heavens, occupy such immense periods of time as to place them beyond all present reach. And we know too little of the duration of our own species on the earth to venture on any assumption thus remote in its fulfilment.

These questions as to nebulous matter in space are deeply interesting, retrospectively, as well as prospectively, in time. Few subjects have so keenly exercised speculation of late as the hypothesis, first sanctioned by Laplace, that our own solar system, with its central sun, planets, moons, and comets, has its origin in the concentration of the matter of a nebulous sphere in successive zones; each several planet being formed by the condensation of vapour at these successive limits in the plane of a common equator; and the satellites being similarly formed from the atmospheres of the planets. It does not annul this theory to admit that there are great difficulties in conceiving the cause of such aggregation of matter at certain points, and of the permanent movements impressed on the bodies thus formed. These difficulties, whatever they be, have not prevented its eager appropriation by philosophers who hold the doctrine of progressive developement according to certain determinate laws, in the creation both of the inorganic and organic world. They find a basis for the evolution or transmutations they suppose, in this hypothesis of the nebular origin of suns and planets; and their argument would be plausible were the hypothesis itself capable of being verified. How far presumptive evidence may reach in future towards such verification, we do not venture to say; but the sources of fresh knowledge are ever opening in this as in other directions of research. The more careful study of cometary phenomena; of the numerous planetoids revolving 
in excentric orbits between Mars and Jupiter; of those meteors, some of which have lately been recognised as periodical in occurrence; and of the aerolites, which impinge in mass upon the earth, - can hardly fail to settle the questions as to the occupation of planetary space to which we have already alluded. How curious, for example, the inference to be drawn from the composition of these falling stones, brought to us undoubtedly from far beyond our own atmosphere, or, as Laplace holdly phrases his belief, 'des profondeurs de l'espace céleste!' Of the various ingredients they are found to contain, every one is familiar to us upon the surface of the earth we inhabit. They represent, indeed; fully onethird of those forms of matter which are still simple or elementary to our knowledge; though under different aspects and forms of combination. Here then we have a sort of material ingress into the regions of interplanetary space; and presumption as to a common origin, though under different modes of aggregation, not merely of those fragmentary masses which casually reach us, but of the great planets also, which move with ourselves in orderly and determined course around the sun.

We are tempted to add one or two other instances here, illustrating the manner in which modern science - resting upon the uniformity of laws, whatever the scale of their operation - has brought evidence to bear upon these vast astronomical questions from the most minute manipulations with matter here below. The happy idea occurred to $\mathbf{M}$. Plateau of Ghent of suspending globules of oil within water, rendered exactly of the same specific gravity by addition of alcohol, so that the globules should be wholly exempt from the action of gravity or other extrinsic force, and free to take any position or motions impresed upon them. By means of a small metallic disk and wires, rotatory movements of various velocity and direction were produced in the 
spherical globules of oil thus suspended in water; making them to assume many conditions closely allied to planetary configuration;- to become spheroids flattened at the poles; - to throw off smaller globules having movements both of revolution and rotation;- - and even rings like those which Saturn shows to our telescopes. These experiments, repeated by Faraday and others, are as valid in the way of inference as they would be were the scale of operation a million times greater. And the same may be said of the second instance we have before us, in those beautiful instruments and inventions of Foucault, Wheatstone, Piazzi Smyth, \&c., illustrating the principle of the stability and composition of rotatory motions, and thereby expounding with admirable simplicity the great phenomena of the precession of the equinoxes, and of the earth's rotation on its axis. The gyroscope of Foucault set into action, and placed on a table, shows to the eye in a few minutes, by the angular deviation from its plane of rotation, the movement the earth has made in this short space of time; - a demonstration almost startling from its simplicity and grandeur. Under the miniature form, almost of a toy, this instrument beautifully illustrates some of the greatest phenomena of the universe.

We have lingered on the subject of astronomy, partly from the striking example it affords of the spirit and aims of modern science; partly from the speciality of its objects, as detached by distance from those relations which so closely connect the sciences treating of matter on our own globe. But though thus distant in space, the vast masses moving in the heavens, and especially the Sun, are variously associated with the matter of the earth, through the elementary forces, if we may thus term them, of which we have already so largely spoken. Here indeed we come again into contact with those arduous questions, where mathematical aids are scantily supplied and few certainties yet attained; but where 
new facts and presumptions unceasingly offer themselves, the foundation and materials of more exact knowledge. Omitting gravitation, which we have already denoted as a special power in the material universe, there comes that wonderful element of Light; blending itself, as we have seen, with heat, electricity, magnetism, and chemical affinity, in such close co-relation of action that we can scarcely dissever its continuity, or detach these physical forces from connection with that great source whence light itself chiefly emanates. The solar beam, as unfolded and analysed in the spectrum, is in truth the most marvellous and mysterious object of the physical world; comprising in itself whole volumes of science, and problems that might put to trial the boldest theorist. The poetry of Milton, sublime though it be, fails, to reach the reality of these great attributes of light, as evolved from a single beam, by simple refraction in passing through a glass prism. It is an analysis of exquisite order and perfection; in which not only are the several colours separated in the same constant proportions, with the intervention of numerous dark lines equally constant in their character; but rays of heat and of chemical power appear severally also at opposite extremities of the spectrum, partially interblended with those of colour, but in greatest intensity beyond the visible coloured limits of the spectrum. We are now speaking only of the simplest relations of the solar light to terrestrial matter; and without any immediate reference to the phenomena included under the undulatory theory of light, which, though attested by mathematicians, and interpreted by numbers, wholly transcend the powers of human conception. We allude, but cannot here do more than allude, to those formulæ of space and time expressing the amplitude and frequency of the undulations, and their variations for the several colours and rays of the spectrum; and the whole series of phenomena of transmission, refraction, polarisation, and interference of 
light - discoveries which have given or added lustre to the names of Young, Malus, Fresnel, Arago, Brewster, Wheatstone, Hamilton, and others scarcely less eminent in this enquiry.

A word or two we must add here as to one relation simple in fact, but not familiar to thought - which light establishes between man and the universe around. The total science of astronomy belongs in origin to this element alone. Extinguish those vivid points or bright surfaces of light which give splendour to the midnight sky;-deprive the astronomer of the feebler rays and fainter gleams which stars and nebulæ, invisible to the eye, bring before his telescope; - and you annihilate at once that science which can predict eclipses centuries beforehand; determine the orbits and return of comets; measure the distances of the fixed stars, and the motion of our own sun and solar system in the universe of space; and penetrate into systems of worlds beyond, where relative degrees of light become the solitary evidence of form and distance. Nowhere are these relations of astronomy to light so well illustrated as in Arago's 'Analysis of the Life and Labours of the elder Herschel,' recently republished in the collection of his works.

Other relations still remain to be noticed here. The phenomena of polarisation, as discovered on the earth, have carried our knowledge nearer to the fountains of this great elemental power; confirming Sir W. Herschel's opinion that light issues, not from the body of the sun, but from a luminous envelope, or photosphere around it. Yet further, the astronomer is able to show, by placing a prism before the object-glass of his telescope, that the light from the fixed stars is submitted to the same refraction, evolves the same colours, and possesses the same velocity as that of the sun, thus more explicitly denoting the sun itself as a simple star in the vast sidereal system. Even the new science of photo- 
graphy furnishes examples of the relations we are indicating. Experiments show qualitative as well as quantitative differences in the chemical effects of light here produced; some of which we may attribute to the terrestrial media through which the rays pass; while others can hardly be explained but by supposing differences in the solar light at its very source, depending on the substance, configuration, or other conditions of the sun itself. The singular changes, periodical or otherwise, ever observable on the surface of this great globe, warrant the belief in such fluctuations of its light, though they may not yet tell us anything beyond this.

But we have yet to speak of other discoveries more recent in date, and illustrating, even more strikingly, these wonderful relations of distant worlds. We allude to those researches of Bunsen and Kirchhoff, which have just established a new method of analysis for metallic bodies - incomparably more simple, delicate, and perfect than any before known to us through the coloured bands, severally produced in the spectrum by flames, in which infinitesimally small quantities of these metals are present. We have not space here to dilate on these remarkable discoveries, anticipated in some part by the researches of Plücker, Wheatstone, and other labourers in this field; but now well defined and opening a spacious path to further enquiry. They have already brought to our knowledge two metals before utterly unknown; and have shown others to exist where their presence had been wholly unsuspected. But the main achievement here is one which forms another link between the earth and its great luminary. By methods of research, equally ingenious and beautiful (founded primarily on the exact coincidence of the coloured bands from the metals with certain of the dark lines of the spectrum, but confirmed by evidence of still higher kind), these philosophers have proved the existence, in the photosphere of the Sun, of six metals at least, familiar to us on 
the surface of our own globe; with the strong presumption that the same methods will multiply these or other analogous results. Discovery of this kind cannot lie dormant, and is indeed already pressing forwards. It is prolific of present inferences, and of promise for the future; and affords the best illustration of that progress and spirit of physical science, which it is our especial object to describe.

Having dwelt thus long on solar light, we may speak more briefly of that other form of elemental force, the Solar Heat, if indeed we can define this as distinct from the former, with which it is so closely blended in passing through space. Endless questions press upon us here, and discovery resolves one only to bring others into view. We have obtained innumerable facts regarding Heat in its various forms, and yet are ignorant of its intimate nature or even existence, apart from the matter by which its action is expressed. In all these questions we have still continually before us the abstract conception of forces or powers, acting in constant relation to each other in the material world - a relation capable in every case of being numerically expressed. The phenomena of light and heat stand peculiarly in this close connection, both in their origin in the Sun, and in all their secondary relations. We may seemingly separate the elements thus named by the intervention of certain kinds of matter, as in the familiar example of the glass screen, or in Melloni's more delicate and complex experiments. But such separation really tells us little more than does the disseverment of light into different colours by refraction through a prism; while all recent enquiries, especially those of Forbes and Melloni, have shown us that radiant heat, in common with light, is strictly subject to those great physical laws which are recognised as the basis of the undulatory theory; the connection being such that heat has not only been polarised and depolarised, according to the terms of this theory, but the phenomena of 
circular and elliptic polarisation have still further confirmed and defined the analogy in question.

The evidences connecting electricity and magnetism, as forces, with the Sun and other bodies of our system, are different and inferior to those which establish the relations of light. Yet are they now continually becoming more numerous and significant. Whoever has seen the star of pure and intense light which bursts forth on the approach of the charcoal points completing the circle of a voltaic battery, or the flood of light thence poured by reflection over wide and distant spaces, cannot but suspect that the new 'fountain' thus opened to the eyes of men (and certainly not destined to remain an idle and valueless gift of science) may be the same in source and qualities as that higher fountain which diffuses light and heat over the whole planetary system. Sir J. Herschel, who ever makes his highest speculations subordinate to cautious induction, has assigned strong reasons for believing the Sun to be in a constantly excited electrical state. The singular phenomena of the tails of comets, he considers as only to be explained by supposing a repulsive force acting from the central body, which force electricity alone could furnish. "The sun electrically charged would induce opposite states in the two hemispheres of day and night on the earth,' is the expression he applies to the effect of such solar condition upon our own globe.* And if we suppose, as may fairly be done, variations in the intensity of this electrical state, we acquire a probable cause for many periodical or secular variations which have hitherto embarrassed science. We allude here especially to changes in the intensity, declination, and inclination of the magnetic force - that extraor-

* These passages will be found in Sir J. Herschel's volume on the 'Nebulæ and Double Stars of the Southern Hemisphere;' a volume in which the tabular results of his vast labours of observation are intermingled with some of the highest speculations to which the human mind has yet legitimately reached. 
dinary power which we are now taught to refer to particular conditions of electricity, in its connection with material media. General Sabine, whom the labours of a life have rendered our highest authority on terrestrial magnetism, has recently furnished evidence, from the exact coincidence in time of magnetic changes or disturbances at remote parts of the globe, that these are due to causes from without, irrespective of local conditions of the earth or atmosphere; while in pointing out the correspondence of certain periodical variations with the several conditions of the Sun, he has shown a direct magnetic relation between bodies thus distant in space. Diurnal or annual changes, subject to this relation, we may in part comprehend; but it needs new elements of knowledge to link together in theory, as General Sabine and Schwabe have seemingly done in fact, the maxima and minima of magnetic variation, with the greater or smaller number of dark spots present on the sun's surface; - a coincidence expressed, on present proof, by periods of ten to eleven years; but one so extraordinary in character, that we are bound to await other periodical recurrences before finally admitting it into the records of discovery.

Meanwhile the Moon also has been found, by delicate observations and averages carefully collected, to exercise a magnetic influence on the earth; - the needle expressing to the human eye certain small variations which strictly correspond with the lunar hour angle. This fact has its peculiar interest in suggesting, and with much probability, a similar influence throughout the whole planetary system, and possibly far beyond. The magnetic conditions and changes of the earth itself come into direct testimony here; - so general and strictly coincident over its surface, as to make it certain that the total globe is in a definite magnetic state; and capable through this state of affecting other worlds, as well 
as the little needle which man makes his index of this mysterious force.

From these vast and remote actions in space around us, we come to those affecting the matter, whether inorganic or living, of the earth on which we dwell. The same great physical forces are still in unceasing action here; with more diversity of effect from the differences of the material acted upon, and from the reflected influence of organic life upon the matter out of which it is engendered. We have already spoken of the impossibility of giving more than a glance over this wide field; but even such cursory view will suffice to show the magnitude of the objects attained in each science, and the energy which is ever active to forward the work:-

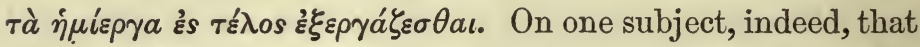
of Electricity, though beyond any other prolific of great discoveries, we need say little here, having in a recent review of M. De la Rive's work described its progress, and the wonderful results thence obtained, as well for pure science as for the practical uses of man. Yet even amidst these marvels of human attainment, it must needs be avowed that on certain points we are still in the very alphabet of Electrical science. The terms of positive and negative, though required for practical use and illustration, are little better than barren phrases as regards any perfect theory of the two polarities; while the whole subject of induction and conduction is still awaiting more certain and complete conclusions than have yet been obtained. That induction is really a certain form of conduction, we have every reason from Faraday's researches to believe. But we have yet to learn what are the states of the molecules of matter which minister to these inconceivably rapid propagations of force? - what the circumstances which render some bodies scarcely capable of this conduction? - what, further, the material conditions which give 
transmission to circuit-currents so vast in length, and in which the opposite polar forces are maintained even though half the circuit be made through solid earth? We can only refer these and other questions equally obscure, to the labours of a future time.

The researches and successes of our own day are the best augury for this future. Many recent discoveries, simple and limited in their origin, have become volumes of new knowledge in their progress. Such are, for instance, the discovery of Oersted, on which depends the whole science of Electro-magnetism; - the doctrine of Electrolysis, as finally established by Faraday, in strict fulfilment of the law of definite proportions; - the further discovery of Faraday, that all matter of whatsoever nature, solid, fluid, or gaseous, is affected in a determinate manner when placed within the sphere of lines of magnetic force; - and his contemporaneous discovery of the rotation of a beam of polarised light under the influence of magnetic force directed through glass of a certain texture; followed by those larger researches (in which Plücker and Tyndall have partaken) disclosing relations between magnetic force and the intimate structure of all crystalline bodies. Many similar instances might be given, but these will suffice for the purpose we have before us.

Some single and unexpected observation may, perchance, furnish hereafter a clue to the truths still desired; and in the beautiful experiments recorded in the Bakerian Lecture and other later papers of Mr. Gassiot, we willingly recognise one avenue through which such research may well be directed. No one can have witnessed the wonderful phenomena of stratified light, as seen in the luminous discharges of a voltaic battery or induction-coil, passing through vacuum tubes of different degrees of exhaustion, without noting in these phenomena the elements of future discovery. The relation of the luminous strata so produced to the electrodes 
or poles of the circuit, and the still more curious influence upon this discharge in vacuo by a magnetic force acting from without, are facts which carry us deeply into that obscure part of physical science, towards which so many paths of enquiry are now laid open and eagerly pursued.

Scarcely less remarkable than the discoveries in Electrical and Magnetic science, are those which regard the material phenomena of Heat. We have already spoken of solar heat and its connection with light, as transmitted to us from the sun. It is difficult to separate this, even in theory, from the heat unceasingly generated or lost in the mutual actions and changes of all matters surrounding us on earth; and science is ever tending to annul such distinctions. The main problem before us, as regards both solar and terrestrial heat, is the intimate nature of Heat itself; whether it be a separate element, or simply a state or condition of matter? The latter definition of it, as a specific mode of motion of material molecules, interchangeable with other modes of molecular motion, we believe to furnish the best interpretation of all the various phenomena; and even of those seeming anomalies of the radiation and focal concentration of cold, which impugned or perplexed all earlier theory. This view, now adopted by the most eminent naturalists, is every day receiving fresh illustrations. The most recent is that furnished by the beautiful experiments of Professor Tyndall, 'On the Absorption and Radiation of Heat by Gases and Vapours;' - a research, the results of which are not limited to this single object, but embrace conclusions stretching far beyond; - even to the distinction of simple and compound molecules, in determining the internal actions, as well as qualities, of the matter by which we are everywhere surrounded. We cannot do more than allude to the foreign labourers in this great field - Ampere, Fresnel, 
Cauchy, Seebeck, Schönbein, \&c.; whose researches give new access to those higher laws of force and motion which we have marked as the ultimate aim of all philosophy.

If seeking to denote briefly the most striking characteristic of modern science in its direction to Matter, we should name at once the principle of Molecular action, as now applied to physical research. Through this doctrine has been made man's deepest inroad into the secrets of the natural world. No single principle is so variously applicable: none has done so much to promote discovery, or to authenticate and give the form and force of law to the results obtained. And yet it may be said to have had a lawless origin, and to have been long the play of human phantasy under the garb of science. We cannot here travel back to those early speculations on atoms, which entered so largely into the staple of the ancient philosophy; and which the poetry of Lucretius has better consecrated to later times than the most subtle prose of the Greek philosophers. In every intermediate age, even the darkest, the atomic doctrine in one form or other has kept a certain hold on the minds of learned or speculative men; - a natural effect of the facility with which it lends itself to any hypothesis, however crude, regarding matter and material phenomena. It was reserved for our own time to render it at once the subject and instrument of legitimate science; the foundation of laws next to mathematical in scope and exactness, and the most powerful of all aids to ulterior research.

This great achievement (for such it is) we owe mainly to Chemistry; and to John Dalton, the Quaker chemist, more than to any one besides. Close approaches had been made before to the doctrine of definite proportions, as represented by the molecules of matter in their combinations. Such anticipations are recorded in the case of every great discovery. But Dalton (speedily seconded indeed by other great 
chemists) first gave clear declaration to the principle; and illustrated its applications, mighty in their universality, with a simple sagacity belonging to the genius and habits of the man. The simplicity of his early experiments is, indeed, characteristic also of the manner in which many of the highest truths in science have been reached. Facts the most familiar to common observation, and thence disregarded by common intellects, have furnished better materials and suggestions for discovery than the most recondite theories.

It has been justly said by Sir J. Herschel that number, weight, and measure are the foundations of all exact science. The atomic doctrine has acquired from chemistry these conditions, which give it substance and certainty as a physical truth. When analysis and synthesis, carefully applied to compound bodies, disclosed a constant and definite proportion of the combining elements, and an equivalent or multiple ratio of parts in every chemical change, the requirements of number, and weight, and measure were all met and satisfied by the discovery.

Here then we have a great law, or group of laws, thoroughly attested; - of high generality; - and proving, because based upon it, that atomic or molecular constitution of matter which alone could afford such results. Whatever name we give to them, these atomic parts exist in all bodies, and determine by their own nature or arrangement the properties and functions of each. That they are minute beyond all human measure is proved, not only by the chemical relations just denoted, but also by those relations to heat, light, electricity, and mechanical force which experiment has demonstrated. No hindrance, indeed, to belief need exist on this score. Matter, either unorganised as we term it, or organised into life, affords endless examples of a divisibility, which we seek to put before the eye in figures, but which no human sense can follow or conceive. While, 
however, even imagination utterly fails to reach the reality, reason accepts this next to infinite divisibility of matter, and the conception of polarities and mutual relations of atoms so constituted, as the sole method of expounding the phenomena. Such subtility of their elementary parts may fairly be stated as an integral necessity in the composition and changes of all the material bodies which surround us.

Had we room here, we might dwell on the astonishing results already derived from this new method of chemical enquiry, through the atomical combinations of matter; and those especially which bring new laws of action and combination into view; such as the doctrines of isomorphism, atomic substitution, homologous series of compounds, compound radicals, catalysis, \&c., which we owe to the labour of Berzelius, Mitscherlich, Liebig, Hofmann, Brodie, and other chemists. Each one of these laws, thus based on the atomic doctrine, is a special example of that spirit of profound research which marks the science of our day; while the growth of organic chemistry, in sequel to labours pursued on this principle, is perhaps the most wonderful of the results thence attained. No surer test of truth in any law than its power of predicting events or effects yet unknown. When, for instance, we find in the different series of organic acids, where every step of change is made in multiple ratios of numerical exactness, that certain void places, left in the first construction of the series, are afterwards filled up by the discovery of compounds answering precisely to the numerical conditions required, we see at once how much has been done towards the deciphering of this, secret scroll of nature. Had the mystical arithmetic of the Pythagoreans and Alexandrian Platonists been converted from a dreamy speculation into sober reality, it would have fallen short of the actual results which this part of science has disclosed to us.

But though especially demonstrated in chemical affinities, 
the atomic theory is far from being limited in application to this single science. We have seen that the other great forces are known to us by their actions on and through matter, such actions and changes, whether from light, heat, electricity, or dynamic force, giving foundation to the several sciences which bear these names. Co-related as they all are with chemical phenomena, we might expect some corresponding relation to that atomic constitution of bodies, from which modern chemistry has drawn its greatest discoveries. And accordingly we find numerous and striking proofs to this effect, furnished by those who are seeking to solve experimentally these high problems, and thereby to establish new connections in the sciences, and laws common to all. We may take as a most instructive example, the various and beautiful phenomena of crystalline bodies, in their relation to heat, light, and eleetricity. The crystal itself, whatever the matter composing it, must be regarded as a substance, the component molecules of which are compelled by a force or affinity (what we may provisionally call polarity) to assume certain definite positions, determining both the inner structure and outer form. The three forces just named all affect most curiously this molecular arrangement. Mitscherlich has shown that while octäedral crystals expand equally in all directions from heat, other crystals, not in this group, change the measure of their angles with every change of temperature. He has further shown that great alterations may be effected by heat in the internal structure of crystals (as in the case of certain prismatic crystals evolving octäedrons under exposure to the sun's heat), without affecting their solidity or altering their external form. The geometry of crystals, indeed, we may fairly say, is but the outer side of the science; the atomic relations and changes within put other and deeper considerations before us.

The fact, now attested in so many ways, that molecular 
changes, transient or permanent, may occur within all bodies while retaining what we call their solid state, is one of high interest, and not enough regarded in its application to every part of physics. The familiarity of some of the instances disguises what is most curious and important in their nature. The simple expansion of a metallic bar by heat involves an atomic change through its every part; less complex, it may be, than those changes of molecular arrangement within crystals. which affect the passage of light through them; but analogous in the main fact of the mobility of atoms, and their power of assuming new and definite position within what we call a solid body. We know from recent experiments that an iron bar is sensibly elongated, and the elasticity of iron transiently, of steel permanently, altered by magnetisation. We know further that the capacity of iron to conduct heat is variously modified under the electromagnetic action. We have the certainty, from the effects manifested at its extremities, that every molecule in the wire of an electric telegraph, whatever its length, undergoes change at the moments of transmission or cessation of the electric force. Without stopping to enquire whether such internal changes may not be interpreted as a tendency to what we term fluidity, we clearly see in them a proof of the individuality of atoms; and very strong evidence that these molecules of matter, minute beyond conception though they be, are endowed individually with axes of motion or polarities, determining their mutual relations, and the changes they undergo when submitted to forces from without. Such conclusions, forced upon us by the simplest view of the subject, are strikingly corroborated by the whole course of modern enquiry; and very especially in those sciences which have their foundation in the actions of light, and the electrical and magnetic forces, upon matter. The time may come when molecular forces or affinities, now represented chiefly in chemical 
actions, may be reduced to a common principle with what we term mechanical forces. And if gravitation be ever submitted to some common law with other powers, such law will probably be founded on the nature and functions

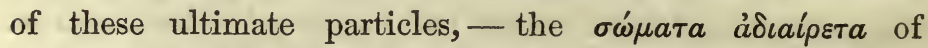
ancient philosophy, - the materials on which modern science works, amidst the most profound mysteries of the natural world.

The whole science and exquisite art of photography, in the relations it establishes between light and molecular changes, gives further evidence that these changes form what may justly be deemed the substratum of all physical knowledge. And the same inference is derived from the discovery of what have been called allotropic states of various substances, as phosphorus, oxygen, \&c.; where an entire change of physical properties is produced, the matter so changed retaining, as far as experiment can tell us, its exact identity of nature. The supposition of an altered arrangement of similar constituent atoms, is the sole present method of explaining this curious phenomenon.

We name these few instances out of many equally remarkable; all expounding, in one form or other, the great principle of molecular action and relation, to the clear conception of which modern science owes so much of its success. Even the points still open to controversy - such as the true nature of the distinction between para-magnetic and diamagnetic bodies (those which take position parallel to the line of magnetic force, or transversely to it) - are clearly seen to depend for solution on more exact knowledge of the modes of molecular aggregation, and their influence on the forces which traverse them. Again, we have the question before noticed, as to the phenomena of the electrical induction through air, glass, and other media; - whether these are due to physical causes yet unknown? or to molecular polarities 
and motions, far removed from all cognisance of the senses, but interpreted to our reason by the closest experimental analogies? Faraday has given the sanction of his opinion to the molecular view of the phenomena, and Grove and others have done much to strengthen this conclusion.

We have hitherto been speaking of Matter generally, without regard to the various aspects under which it is known to us. For with all the refinements of modern analysis, there still remain more than sixty substances undecomposed, and which must therefore be deemed simple or elementary to our present knowledge. Of these the largest proportion are what we term metallic bodies, and most of the additions recently made to the list of simple substances belong to this class; with the further curious speciality pertaining to several of them, that, while perfectly distinct from all others in physical characters, they are hitherto known to exist in a few rare specimens only. Almost we might be tempted to surmise that they belong to the number of those materials of which aerolites seem to tell us that other worlds are made; and that they exist more largely in these worlds than in the feeble representation of their existence on our own globe. Such suggestion, however, must be received simply as illustrating the manner in which modern science attaches facts already attained to problems yet unresolved; concentrating them as it were around common foci, towards which they ever more closely converge.

The great problem regarding these many kinds of matter on our own earth, lies in the question, whether they may not hereafter be lessened in number by reduction to certain elements, common to several or all? Whether, in other words, bodies simple to our present knowledge, are not actually compound in their nature? Chemistry, it must be owned, has hitherto done little directly towards solving this question; 
the vast resources of analysis having tended to multiply elements upon us, rather than to abridge their number. Some approach in this direction has, however, been made through the law of isomorphism; which, in showing relations of mutual substitution between certain elementary bodies having other curious resemblance of physical properties, has led to their arrangement in groups; preparatory, it may be hoped, to some future discovery which will give a common basis to all the bodies thus related. The most remarkable of these groups is that comprising chlorine, iodine, and bromine. Arsenic and phosphorus - selenium and sulphur - and the group of the platinum metals, are other examples of these singular relations, to which, in connection with the law of definite proportions, the labours of the chemist are sedulously directed; not solely for instant results, but with the prospect constantly before him of those higher truths, to which some one single discovery may perchance open the way. The present methods of chemical enquiry are peculiarly fitted to this critical examination of the simple bodies; while in the spectrum analysis - that extraordinary discovery of which we have spoken - we find the promise of results scarcely yet compassed even by the imagination. Electricity, again, equally powerful and delicate as an instrument of research, has been, and must ever be, an especial aid in the prosecution of an object worthy of all the labour that can be given to its attainment.

We have already spoken at some length, of Light as an element in the universe; and of its properties as it comes transmitted to us from the Sun and other worlds in space. But much more might be said, had we space for it, of those wonderful phenomena - whether derived from solar or artificial light - which have exercised the highest genius of modern science, and largely invoked mathematics as an aid to experimental enquiry. If we have not yet reached a true 
and complete theory of the spectrum, or learnt the many conditions under which light penetrates, pervades, or even becomes latent in different forms of matter, this deficiency is due, not to any want of zeal in research, but to the intrinsic difficulties of dealing with this subtle and multiform element. Looking at the colours alone of the spectrum so beautifully defined to the eye by the several refrangibilities which evolve them - controversies still exist as to the manner in which they combine with each other; and until the recent suggestion of Sir J. Herschel, followed by the very valuable researches of Professor Maxwell (1861), which rescue green from its subordinate place as a compound of yellow and blue, we were at fault even as to what might be deemed the primary colours in the scale. The connections of another portion of the spectrum, with those changes in the molecular conditions of matter which we call chemical, furnish a new avenue to the higher physical laws which lie before us. In photography, the exponent of these changes, we have an agent so working as almost to put to shame the manipulations of human art. To fix what we transiently see by reflection from a mirror, was the object sought for, and for a long time ineffectually. Once attained, every year has added to the beauty and perfection of an art, which may especially be called the child of science, and which we must not yet affirm to have reached its maturity. Whoever gazes on the colours of the solar spectrum, cannot but be seduced into thinking that photographic chemistry may hereafter afford colouring to our pictures, as well as the simple imagery of light and shade. We are still too imperfectly acquainted with the complex machinery of the solar beam to warrant abandonment of the object; even were it not probable that the phenomena of colour are mainly due to the atomical constitution of the bodies recipient of light, and to the organic structure of the eye itself, forming the last material link with. 
the percipient being.* This, in truth, is one of the cases now frequent in science, where even failure may become a fountain of discovery. Negative conclusions have their value as well as positive ones, and often form an index to the path through which truth and certainty are finally reached.

Without going into details, we may allude here to the recent photo-chemical researches of Bunsen and Roscoe; to those of Becquerel on phosphorescent and fluorescent bodies; and, still more, to the remarkable experiments of St. Victor and Grove; all showing the direct action of light upon the molecules of matter to be far more universal and minute, as well as more definite and lasting, than was before dreamt of in our philosophy. In man, it has been the general presumption that light finds ingress by the eye alone, and with the sole effect of giving instant vision of things without. But recent enquiry calls upon us to recognise, even in the eye itself, the retention and probably reflex actions of light within the precincts of the organ; affording, through what we may venture to call photographic impressions on the retina, the only plausible explanation of its subjective functions, and of other phenomena little heeded from their familiarity, but presenting problems of the highest interest to philosophy. The experiments of D'Arcy prove that the impression of light is often retained on the retina for fully two and a half minutes, - the time in which a luminous particle or undulation passes through nearly thirty millions of miles of space! What is the condition of Light - be it conceived as matter, or motion, or force-when thus arrested and enchained in a living organisation? In this brief question lies one of the most profound

* The curious phenomena of colour-blindness afford illustration of this remark. This subject is treated of by Sir J. Herschel in a recent Memoir; in which also, with his wonted ability, he revises generally the mutual relations of the prismatic colours, and presses upon the notice of the experimentalist the important distinction between the study of pigments or negative colours, and prismatic or positive ones. 
of the problems to which we have just adverted. The older theory which regards light as an emission of material particles, was called upon to answer the enquiry, what becomes of matter thus absorbed? - while the undulatory theory, now accepted as the great law of Light, has to explain what becomes of motion? In the latter theory, closely linked as it is with the doctrine of convertible forces, we undoubtedly find the easier solution of the problem. But in this part of science more especially, such questions are perpetually pressing upon us, each fresh fact (and the simplest are often the most suggestive) giving access to objects of more advanced thought and speculation.

In passing thus cursorily over the sciences which deal with the various forms of matter on our globe, and the forces affecting them, we have said nothing of that Science now become so vast in its objects and methods, which takes as its province the outer structure of the globe itself; and the changes, organic as well as merely material, succeeding one another for ages on that surface which is now the dwellingplace of man. Such seeming omission we may explain by reference to previous Articles of this Review, in which the present aspect of geological science, and the questions it involves, have been considered at some length. In no one department of Natural knowledge, we may safely affirm, has greater zeal and activity been displayed, or with larger and more successful results. We must repeat further that Geology has (within the last thirty years more especially) undergone a change which raises it far above the mere history of the succession, location, or dislocation of strata, and connects it inseparably with other branches of science still more fruitful of discovery. Fossil Geology in particular, the creation of our own time, affords a striking example of what may be attained by zealous research, submitted to 
methods of sound and careful induction. This science has given us deeper insight into the natural history of our globe than any other can supply; and, taking order of succession as its basis of enquiry, has furnished more certain means, through fossil remains, of identifying strata in distant parts of the earth's surface, and what is a far higher attainment, of determining the forms of organic life, during ages long anterior to all human calculation of time. This is not merely a new branch of knowledge, but almost an ingress into a new world. Nor can it in any sense be deemed a stationary science; while vast regions still remain wholly unexplored; and while occupied, as it continually is, not solely in discovering new fossil species, but with the higher object of determining the origin and succession, in the series of rocks, of those typical forms of life which have descended to the Fauna and Flora of our own day.

This subject of Palæontology is indeed allied in every part with the present history and physiology of animal and vegetable life; - that great domain of knowledge which, though closely encircled round by physical laws and phenomena, and approached only through these, has still a secret region within, - the origin and principle of life itself, hitherto inaccessible by any method of human enquiry. It was our design to have included Physiology among the several subjects of this article; as illustrating not less than other branches of science-in some points even more strikingly - the advances made in actual knowledge, and the spirit which impels and animates to further research. While admitting that this spirit has sometimes run riot upon questions, the very mystery of which invites and emboldens speculation, we find true inductive science moving steadily onwards amidst these more erratic courses, to those truths - the $\kappa \tau \hat{\eta} \mu a$ 's $\dot{a} \varepsilon \dot{\text { - }}$ - which are the certain reward of all legitimate inquiry. So much, however, has recently been 
attained in animal and vegetable physiology at large, and very especially in the great department of Human Physiology, that not even the briefest summary could bring the subject within our present limits; and we must postpone to another time the notice of these eminent discoveries, and of the works which best describe and illustrate them.

What has been derived, however, from other branches of physical science will, we trust, adequately fulfill our intention of showing in what spirit all such science has been recently pursued; and what signal success it has obtained in compassing and expounding the great phenomena of the natural world. Happy those who can work tranquilly amidst these wonders of nature, animated by the love of knowledge for its own sake, and undisturbed by the storms which are ever agitating and vexing the world without.*

* It is always interesting, as well as important, to learn the different methods by which men of genius arrive at a common truth. When the first edition of this volume was published, I knew but by name only the remarkable writings of Dr. Mayer of Heilbronn (appearing under various dates from 1842 to 1851), in which the doctrine of the mutual convertibility of certain of the physical forces is maintained by bold and ingenious argument; - anticipating in part those inductive proofs, which have now given it the character of a great physical law. His earlier views chiefly appertain to the equivalent relations of Heat and Mechanical Force; but in later memoirs he extends his argument to other forces, including those which act in the phenomena of organic life. 


\section{LIFE AND ORGANISATION.*}

[EDiNBURGh Review, January 1859.]

TN a former number of this Review (Jan. 1858), we took 1 occasion, from some remarkable works then before us, to comment on those present conditions of physical science which more especially mark its progress onwards, and the larger scope and higher spirit now given to its pursuit. Our view, however, was then confined almost exclusively to the inorganic part of creation; - to those sciences which treat of matter unendowed with life, and of the great natural forces or powers which we recognise by, and through, their various action on the material world.

We have now before us another series of works (to which many more might be added) recording the present state of our knowledge of matter organised into life; - of that vast domain of animal and vegetable existence which lies around us; presenting a thousand problems to our reason, and almost appalling contemplation by the multitude of its objects. This short and seemingly simple word - Life - does, in truth, in

* 1. General Outline of the Organisation of the Animal Kingdom, and Manual of Comparative Anatomy. By Thomas Rymer Jones, F.R.S. (Second Edition.) London, 1855.

2. On Parthenogenesis, or the Successive Production of Procreating Individuals from a Single Ovum, from the Hunterian Lectures on Generation and Development for the Year 1849. By Richard Owen, F.R.S., \&c. London, 1849.

3. The Rambles of a Naturalist on the Coasts of France, Spain, and Sicily. By A. de Quatrefages. 2 vols. 1857.

4. Sea-side Studies at Ifracombe, Tenby, the Scilly Isles, and Jersey. By George H. Lewes. 1858.

5. The Master-Builder's Plan, or the Principles of Organic Architecture, as indicated in the Typical Forms of Animals. By George Ogilvie, M.D. 1858. 
itself include the greatest of all the problems submitted to human thought. All distinctions and diversities are trifling in comparison with the one line, which separates inanimate matter from those living organisms created out of it; - with properties and powers of endless variety; and, above all, endowed with that wonderful power of reproduction, which maintains the continuity of the species while individual forms are successively passing away. No step so vast as this, no mutation so wonderful, in any part of creation. The mystery is not solved - scarcely lessened to our conception - by those researches which, descending in the scale of existence, seem to obliterate all certain distinction between animal and vegetable life, and to bring the latter to the very lowest grade to which the term living can fitly be applied. It is still the distinction between that which can reproduce itself and that which cannot; and in this single condition lies the clearest expression of all vitality, whatsoever its form or degree. No definition of life can be complete without it. Alone it suffices to mark that line of division which even the finest microscope fails to reach; and it applies no less to that more wonderful and complex animal machinery by which the higher forms of existence are maintained and perpetuated.

Into this domain of organic life modern science has penetrated with not less zeal and success than have signalised it in the other branches of physical science, notwithstanding certain distinctions which may seem to favour the pursuit of the latter. Such are the surpassing grandeur of the various discoveries in inorganic nature; - the mathematical certainty of many of the laws thence derived ; - and the important practical uses to which these discoveries have been applied, enlarging the dominion of man over nature through elements which were formerly known but as objects of admiration or terror. No period has been so prolific of these achievements as that in which we are now living. 
On the other side, however, we find numerous incentives to a like zeal in the study of the living existences around us. The simple presence of the attribute of life, as we have denoted it, tells for much with every reflecting mind. But this part of natural science gains also by the comparative facility with which it may be successfully pursued. Few can compass all that is required for experimental research, especially under those refinements of method which have now become essential to success. Many are competent to a science chiefly of observation;-amidst objects present to the senses, often associated with the charm of natural scenery, and consonant to the natural tastes and habits of the mind. The traveller who gathers his unknown plant in Australia or Paraguay; the naturalist who discovers some new form of animal life, or disentombs some fossil from its rocky sepulchre of ages; the physiologist who detects new organs or instincts in animals already known - all hold rank, in one degree or other, as labourers in this great field. No fact so small as not to find a place in the volume of natural knowledge.

In thus distinguishing, however, the two great objects of scientific pursuit, it must be kept in mind that no strict line of demarcation exists between them. The progress of knowledge is ever bringing more closely together, and under the dominion of common laws, facts and phenomena apparently the most remote. Though rejecting the modern phrase of ' unity of science' as a vague effort of language to reach an ambiguous truth, we see and admit a constant propensity towards unity in a more qualified sense. Facts multiply every day in number, but every day they are submitted to new conditions of order and comparison. Phenomena familiar to the senses from the earliest ages of human records, are expounded to the reason by the discoveries of our own time. Life itself, taking the term in its simplest sense, can be interpreted only by the laws which pervade all matter; and is 
unceasingly subject to those great elementary forces - gravitation, heat, light, electricity, and chemical action - which are ever in operation around us. They are the instruments in those wonderful organisations which it has been the will of the Creator to bring into being; and they have subordinately become instruments in our hands for interpreting these higher manifestations of the creative power.

It would be impossible within the limits of this article to analyse the several works prefixed to it, or even to denote the especial objects and merits of each. It will be seen that several of them relate more especially to those lower forms of animal life which lie close to the boundary - if certain boundary there be - between the animal and vegetable world. This particular portion of natural history has of late risen into high popularity, and the works devoted to it display an exuberant enthusiasm in the research. While the astronomer is soberly dealing with the great elements of space and time, which make the material of his science, the modern naturalist is rioting in rapturous language about the beauty of his zoophytes, and the microscopic marvels of infusorial life. The real beauty of many of the objects, the scenery with which they are often associated, and the curiously delicate methods of research employed, will explain in some part this ardour of pursuit. Nor can we deny a sentiment of grandeur as belonging to objects even thus minute, when so infinite in number and variety. That which human calculation cannot reach, has in itself a certain element of the sublime, be the subject what it may. But, connected with this study, we have also the many mysterious questions which regard the manner of generation and existence of these simpler forms of animal life, and their relation to other beings of higher order;-topics well fitted to take strong hold on the mind of every thoughtful man.

On some of these questions we shall have to speak hereafter. 
Meanwhile, we must explain in reference to the subject of this article, that although we have it in view to indicate the progress and spirit of modern science in its researches into organic nature, we must limit ourselves mainly to Animal life; referring to that of the vegetable world only in illustration of the former. Even under this limitation we cannot go into anything like a complete review of the topics embraced under the name of Animal Physiology. Whoever takes up the massive volume of Dr. Carpenter-a work of great excellence, and the most complete we possess - will see how much is comprised in this wide domain, how profound the subjects offered to human thought, how large the voids yet left for future enquiry. What we may reasonably attempt, is to place before our readers a summary view of such questions and discoveries in this part of science as are of highest import in themselves, and best indicative of the scope and spirit now given to its pursuit.

It will be obvious, even to those who give only casual thought to these questions, that many of them tend to take a metaphysical form; while not a few are of a nature wholly to transcend the present faculties of man. The attainments actually made by these -faculties, in their more exalted use, furnish reasons for not drawing hasty and arbitrary lines in limitation of further progress. But certain barriers there are which the highest genius is the first to recognise and submit to ; seductive though the speculations may be which here first press upon the reason. Not less boldness and mental power are shown in well defining the boundary of research, than in adventuring wantonly over and beyond it.

What we have already said on the abstract principle of Life, as appended to material organisation, will show that this, the great and elementary point with which we are here concerned, comes under the class of questions just spoken of. Notwithstanding all that has been done - and it is vast in amount 
and variety - towards our knowledge of living beings, and of those organisations upon which vital functions depend and by which they are maintained and reproduced, the question unceasingly recurs, and can in no way be put aside; - What is the principle or property - if any - superadded to the known properties of matter, giving it those new conditions which create and constitute vitality? It is this enquiry which, in one form or other, has exercised every age and school of philosophy; and been argued the more intently, or even passionately, from the question having been often made to embrace intelligence and the other mental functions, as well as mere vitality. Under this latter aspect, it will be recognised as that old problem of Materialism, upon which so much argument has been wasted; - a controversy equally fruitless in all time to come, since no human conception can reach the abstract nature either of matter or mind; nor any argument show that things perceived by the senses have more independent reality than the principle perceiving, and the intelligence and volition acting upon them. The materialist fancies himself on firm ground, because his argument has Matter for its foundation. His matter itself is known only by and through that mind which he assumes to create out of it.

On this point, and for these reasons, we do not dwell longer; but rather proceed to that part of the subject, more accessible to human reason, which engages at this time the earnest attention of naturalists in every branch of their science; viz., the manner and extent of influence of the great physical forces ever in action around us, in producing and maintaining those other powers and properties which we call Vital; and which, in their aggregate, represent all we can define as Life upon the globe. It is clear that these great powers, Heat, Light, Electricity, and Chemical force or affinity, whatever their nature or mode of developement, stand to each other, in their action on matter, in the relation of 
mutual convertibility; - that their forces, however altered in respect of action, are never really lost or lessened; - that they are the efficient energy, not solely in the greater and more obvious phenomena of the material world, but equally so in the most minute molecular actions to which matter is subject. We can modify, by human powers and machinery, the aspects of force and its actions upon matter. We can never either create or annihilate it. These conclusions, at the utmost but vaguely and partially surmised before, have now acquired certainty enough to give them place among the great general laws of nature; and experimental science is every day bringing fresh facts to their proof and illustration. Whether the term of 'Co-relation of Forces,' provisionally applied by Mr. Grove to describe what is our present knowledge, may not hereafter merge in the single phrase and conception of Force, as contradistinguished from the matter on which it acts, is a point open to future determination. Mutual convertibility is closely akin to unity, if not an actual expression of it. Much that is of the deepest interest to philosophy hangs upon the solution of this problem; involving, as will be seen, all the relations of matter to that mighty influence which has been destined to mould it into form, activity, and even into life itself.

It is here, in fact, that we find ourselves in the very heart of the question to which we have just alluded as still the subject of serious controversy; viz., whether there be really any separate Vital Principle; a positive and independent power, giving organisation and life to certain combinations of matter?-Or whether the simple vital phenomena may not be referred, as effects, to those great physical powers, which we see acting so incessantly on all matter in the universe; and the actual influence of which upon the vital functions is obvious at every moment of existence?

Each of these views has found zealous advocates, and been 
discussed in the bold and free spirit which belongs to the science of our day. Each, in truth, furnishes ample materials for difference and dispute. Looking at the controversy as it now stands, we find the latter opinion to have gained much upon its adversary. The doctrine of an independent vital principle is one of old date; and in its very nature admits of little argument or advance. It rests mainly on the assumption that the phenomena of life, even in their simplest form and apart from mind and intelligence, are unlike, and incongruous with, any actions of which we are cognisant as the obvious results of physical forces operating upon matter. Though the argument may be varied in form, yet in no way can it be made more absolute, or stretched beyond this method and degree of proof. By the very terms of the question, we quit here the region of the senses and of material experiment, and affirm a power unknown, except in what we presume to be its effects. It is negative evidence; and, as far as we see, can never be rendered other than such.

Those who advocate the other view, adopt a doctrine equally insusceptible, it may be, of positive proof; but yet constantly progressive, and in its progress prolific of results favourable to the conclusion sought for. They have the advantage in the very outset of being able to affirm, that without the action and influence of the physical powers in question, no life could possibly exist. The wonderful discoveries recently made as to those more subtle actions of electricity, heat, and light, which evaded the grosser experiments of former times, have assisted their argument. Equally so the researches, not less wonderful, into the molecular constitution of bodies; and the relative proportions in which such molecules, whatever their nature, unite in every case of chemical combination. Chemistry, in fact, and especially the chemistry of organic bodies, has done more for us in deciphering the structure and functions which appertain to life, than any of the other 
powers of physical analysis. The definite proportions which exist in every union of the simpler chemical elements, are found also in the most complex compounds which form the material of living bodies. The poisonous ingredients of animals and plants, equally with their nutritive portions, yield invariable results to organic analysis. Various specific secretions familiar to us in the human economy, exist, under the same chemical composition, in the lower animals, even as far down as insect life. A minute quantity of phosphorus is detected in the nervous substance of the brain, bearing a certain proportion to the other ingredients, and showing thereby its determinate relation to this wonderful part of the living economy. Endless similar instances might be given, to prove the extent and uniformity of the chemical actions which pervade every living texture; equally definite in all which concerns its growth and-maturity, as in the changes which precede and produce its final decay.

But modern Chemistry goes yet further in its aims and success. Numerous substances, both animal and vegetable in kind, known to us before only as the products of living actions, have been actually produced in the laboratory; identical in every chemical character, but subject, we must add, to this notable distinction; that whereas in nature the series of living organisms is begun from the combination of a few simple elements, no present artifice of chemistry can fully imitate this higher workmanship, otherwise than by acting on compounds already formed. In theory, however, it does not seem improbable that this ultimate step may yet be made. Whatever experimental skill, aided by boldness of aim, can accomplish, will be done by those who now work in the physiological department of chemistry. The schools of Liebig, Dumas, Hofmann, Bernard, \&c., are creating pupil s and fostering a zeal in the pursuit, to which we may well look for results hitherto unimagined or unattained. 
We may dwell somewhat further still on this argument of the relation of the great physical forces to vital phenomena, inasmuch as the question is really supreme among those which relate to the theory of life. It is obvious as a principle in conducting it, that we must proceed upwards from the lowest and simplest forms which occur in the scale of being. If in these the properties of life and reproduction depend on physical agents alone, without any new and unknown principle of power being added, we must needs carry the conclusion onwards to higher grades of organisation. No line of limitation exists, obvious either to the senses or to reason. Some might conjecture it to lie in the distinction between animal and vegetable life. But, even acquiescing in all that is expressed in this distinction, can we fairly claim for the Medusæ or the Oyster a peculiar cause or principle of life, which we deny to the Sensitive plant, the Dionæa Muscipula; or to the common Nettle, Berberry, and various other plants, each endowed with some peculiar sensibility or motive power? That the same natural forces have influence on all these organisms is certain. Are we to suppose some mysterious agent, yet unrecognised, as needed in addition to explain the appropriate functions of animal life, even in their simplest form of organisation?

This particular question is obviously subordinate to the larger one we are now discussing. The grounds of argument are the same, the difficulties exactly alike. Thus far we have dwelt more especially on chemical action, as giving us closest access to the laws of vitality. But Electricity, that wonderful agent on our own globe, and probably throughout all space - which, scarcely known a century ago, is now the most powerful instrument in the hands of man - bids fair to become an equal exponent of the vital functions, and especially of those important functions which belong to the ervous system. We shall have occasion to recur to this 
point, and merely mention it here, as relating to one of those great powers which are incessantly acting upon and through life in all its forms. The same may be said of Heat; the influence of which, in promoting organisation and maintaining the vital functions, is familiar to us in a thousand ways, and attested in more scientific form in every part of animal physiology. The action of Light, as separate from heat, is somewhat more ambiguous; but that it has special effects on these functions cannot be doubted; and very remarkable proofs of this are every day multiplying upon us. We might almost deem sufficient as evidence, the spectacle of the sudden bursting forth of life of all kinds under the influence of a bright summer sunshine. But science goes far beyond this, in showing that Light, like Heat, does truly permeate and act upon those molecules of matter, of which all bodies, organic or inorganic, are composed. Much is yet to be learnt on this curious subject.

Such are the general evidences and arguments of those, who believe that we need look for no other vital principle than lies in some modified function of the great forces which we see in unceasing action around us, and feel to influence at every moment the conditions of our own being. The fact already noticed of their mutual convertibility, and other various proofs that force may be hidden, latent, or altered in aspect, but never effaced or lost, undoubtedly favour this view. When its sensible effects disappear, we have cause to believe that it is either operating in some way too minute for our detection, or that it exists in a latent condition ready for some new form of future developement. The advocates of this doctrine are apt to startle us by their bold illustrations. We feed a jaded horse on a peck of oats, and he is able to travel again; - the effect, say they, of the evolution and conversion into nerve force, of that power which has been laid up in the grain during its growth. We light and warm 
ourselves, and give propulsion to our engines, with that coal which for countless ages has retained within its substance the light and heat of its original forest growth. Such instances as these may seem rashly to outrun the cautious step of scientific induction. Yet they find authority in the fact, well authenticated by Faraday, that one drop of water contains, and may be made to evolve, as much electricity as under other manner of evolution would suffice to produce a lightning flash. And we might quote as an instance not less wonderful, and still more in point, that germ of vitality preserved for twenty or thirty centuries, which can make prolific, under our own eyes, seeds taken from the mummy cases of ancient Egypt. When positive observation teaches us this much, we are not in case to deny the analogous conditions put before us for belief. The abstract conception of force, thus laid up for future evolution under the same or a new form, is one of the most profound upon which either reason or imagination can dwell.

We must not, however, linger further on this question, fundamental though it be to all researches into the nature and laws of life; and blending itself with every subordinate question in which these laws are concerned. If it seem that we have pressed the argument too much on one side, we must repeat that the doctrine of a separate vital principle rests on negative grounds only, and little admits either of amplification or detail. The bold and active science of our day has for the most part ranged itself on the opposite side; and is ever occupied in fixing new relations and equivalents of power - the materials, it may be, of more general laws than have yet been reached by human intelligence.

The question we have been discussing is common both to animal and vegetable life. We now come to other topics, subordinate and more special in their nature, yet all of high interest to natural science, and all demonstrating the spirit 
and zeal of modern enquiry. The first of these topics has been already partially noticed; viz., the relation to each other of these two great natural provinces, each so profusely peopled, and each exhibiting such wonderful design and exuberant variety of the creative power. This question has of late been closely examined by naturalists. It involves the fact, in itself one of great interest, that in the lowest and simplest forms both of animal and vegetable life, there is so close a coalescence of the two, as well in structure as in mode of developement, that it is often difficult to say to which the individual belongs. Even the acute microscope of Ehrenberg put down as Polygastric Infusoria what are now discovered to be germs of vegetable life. We are brought here, in fact, to that doctrine of our own time, that the simple cell is the primitive germ of all living organisation, even of that which in its end attains the highest grade of animal existence. On this subject we shall have to speak hereafter. Meanwhile, looking simply at the two great kingdoms of life, as they diverge from this initial point by a gradual scale of ascent to higher states of each, the special question arises; - What are the peculiar physical conditions which separate and severally distinguish them? Of the answers to this question all may be said to converge more or less towards one point; - viz., the fact, well established, that while vegetable life is created and supported from unorganised or disorganised matter, animal life always requires for its nutriment matter already organised either by its own or vegetable processes. It cannot, so to speak, work the raw material into its own texture. Even the mere animal jelly, floating in water without obvious organisation, is nourished by absorption of vegetable sporules, or animal matters so comminuted as to serve to this end. A more special distinction, but equally explicit, has been drawn from the chemical action of plants on the atmosphere. Expressing it in the words of Professor 
Huxley, 'Wherever an organism is found to decompose carbonic acid under the influence of sunlight, and to set free oxygen, that organism may be ranked as a vegetable, however active may be its movements.' Others have been suggested (such, for instance, as the curious fact that no living being has a form geometrically regular, or shows other than a curved configuration of its surfaces): but none, hitherto adduced, are so striking or unequivocal as those to which we have just adverted.

We have before stated our design to limit the present article chiefly to those researches into Animal life which have been so prolific of discovery, as well as of speculationoften profound, sometimes rash - on this higher part of creation. The same reasons which lead to this limitation, will oblige us to take up these topics in a somewhat desultory manner; with less regard to their order and completeness, than to the interest they possess, or the illustrations they afford of the progress lately effected in this part of science. The two most remarkable facts attesting this progress are, undoubtedly, the extraordinary additions made to our knowledge of existing species, nearly quadrupling their number within half a century; - and the discovery of that vast and heretofore hidden world of extinct animal life, which has been entombed, for ages beyond all human count or speculation, in the rocks that cover our globe. The ardour of the traveller and naturalist, aided by the microscope, has rapidly multiplied to our view the species of present animal life. The equal ardour of the geologist, working amidst the strata, which chance or labour discloses to him, has shown what we may well call a series of successive worlds of animal and vegetable life; since, though the general types be the same as those in present existence around us, the species differ more or less in each of the successive periods of time, thus wonderfully brought to light. 
Of the two great steps in knowledge here denoted, the latter is doubtless the most remarkable, and replete with problems of the deepest interest; including time as one of its foundations, and thereby bearing on the history and destinies of Man himself. But the extraordinary multiplication of the number of recognised living species, though less striking to the imagination, yet.furnishes conclusions hardly less important to the philosophy of life. It is difficult indeed to define, even approximately, the amount of this multiplication, which occurs chiefly, though by no means solely, in the lower parts of the animal series. The powerful eye of the microscope has shown, in earth, air, and water alike, new forms of life, invisible to all unaided sense, but endless in aspect and variety. Every bucket of water taken up from mid-ocean teems with vitality. The dredgings of Forbes and others in shallower seas show different zones of depth, tenanted by different species of animal life. Even the bed of the Atlantic, 10,000 or 12,000 feet below the surface, was found, in soundings for the electric cable, to be covered with the remains of Foraminifera which, for aught we can tell, may have lived at this depth. We all know (and in hot countries cogently feel) how thickly the air is peopled; not merely with the birds which crowd and ornament our museums, but yet more with incalculable swarms of insect life, even more audible than visible to sense. The tropical forest is noisy day and night with the life it contains. The sea is luminous with animal phosphorescence. Nearly two hundred species of glow-worms, and forty or fifty of fire-flies, are catalogued as luminiferous animals of the land. The researches of Ehrenberg, eminently successful among the fossil and living Infusoria, have since been directed to the atmosphere; in which, by appropriate methods, his microscope has detected numerous other minute species heretofore unseen and unknown; yet not indifferent, we may well believe, to those 
higher animals, even the highest, which breathe air on the surface of the globe.

Another result of recent enquiry has been our increased knowledge of the Entozoa, and of Parasitic life generally, both animal and regetable in kind. No natural phenomena are more curious than these. A few examples, seemingly rather deviations from nature than a part of it, formerly comprised all we knew on the subject. Now we may fitly term it a branch of natural history in itself, so numerous are the instances, so definite the relations it involves. When we find even the earth-worm haunted by a parasite (the Gregarina) living within and upon it; and numerous mollusks and insects, with peculiar entozoa pertaining to each, we gain some idea of the extent and singularity of these relations. It is not an anomaly we look upon, but a part of creative intent; - an expression of that great design which makes organised life in one form everywhere subserve to the maintenance of another. Exception may perhaps be taken for those species of parasites which are found in diseased animal textures, and in such alone; as we recognise them in morbid states of which man himself is the subject. These curious cases have been considered to sanction the hypothesis of equivocal generation, of which we shall speak hereafter - the disease itself being regarded as antecedent, and the parasitic life as growing out of it. It would be difficult to bring positive proof on this point. It is one reserved for future research; as are those strange analogous phenomena which seem to show that the same ova or parasitic germs, transferred to different organic textures, are capable of evolving different forms of animal life. Minute though these objects be, and inaccessible to all unaided sense, there is no part of natural history which strikes deeper roots into the secrets of the living creation.

Rising higher in the scale of existence, we find in all the 
classes into which zoologists have distributed the animal kingdom, the same astonishing augmentation of numbers; less indeed as we reach the higher classes, but even among the Mammalia trebling the number within the time we have named. The birds and fishes made known to us have been still more multiplied; while of insect genera and species, the ratio of increase is such, that calculation can hardly follow it. A commission sent to Brazil for beetles alone, is sure to bring back from that country, so profusely swarming with animal life of all kinds, many species before unknown to the European naturalist. The cases of our Museums are replete to overflowing with these new insect forms from every region of the globe; certainly far exceeding 100,000 in their total number. The British insects alone have been catalogued up to 12,000 : - the Coleoptera in the collections of Duport and Baron De Jean, at Paris, amount to more than 20,000 species. Of the Butterfly at least 3,000 species have been enumerated; while the catalogue of the Moths almost transcends any numerical estimate.

This augmentation, however, must be qualified by the certain fact, that numerous individuals have been named as species which are not really such. The species has been recorded, where it is only the specimen or variety we have in our hands. How far reduction may be carried on this score, it is not easy to say. Undoubtedly it must be largely admitted hereafter in correction of our actual catalogues.

But we take an imperfect view of life, as it exists on the earth, if attending only to the number of species, vast though this be. The numerical individualities of different species their richesse effrayante, to borrow a phrase of Cuvier-is yet more impressive to the reason and imagination. A single swarm of insects, or a shoal of herrings, would in simple numbers represent a populous empire. Without wishing to malign a popular English institution, we might quote the 
white-bait dinners of a Greenwich season, as affording some practical idea of the numerical demand which the human kitchen makes upon one rare species. And what is this to the amount of life which the whale imbibes and annihilates at a single draught? What estimate of numbers can we put upon the flies of Egypt, or those continuous clouds of locusts, which for days together darken the sky, and devastate the fairest regions of the earth? Instances of this kind might be given without end; each recording the same marvellous profusion of individual life, made more wonderful by the rapid succession of generations as we descend in the scale of being. Take the single instance of the Aphis. By the most certain calculation of the rate of production in this minute creature (weighing scarcely the $\frac{1}{1000}$ of a grain), it is found that the successive generations from a single Aphis in one warm summer, might amount to a quintillion of living beings; - a number so much above all human comprehension, that it offers but a vague row of cyphers to the eye. Or take Ehrenberg's estimate of the 140 billions of infusorial animalcules contained in two cubic feet of the Tripoli slate of Billin, of which rock their siliceous cases form the substance; a wonderful aggregation of individuallives, however we may value the grade of being to which they belong.

This marvellous multiplicity, both of individuals and species, is expressed even by the manner in which the destruction of life takes place on the earth; and by the vast proportion of the germs and ova of forthcoming life, which never reach maturity. Throughout a great part of the animal creation, there is a strict inter-dependence for that aliment which one species affords to another; and a constant pressure or struggle, greater or less in degree, to satisfy this necessity. It is the same where vegetable aliment alone is concerned; the effect in each case being a continual annihilation of life; - sometimes of species as well as individuals; - and most 
abundantly of those germs of existence which in the lower parts of creation are evolved in such countless profusion. The total condition of organic life, from its lowest to its highest grades, is modified or controlled by these several causes, unceasingly in action over every part of the globe.

Our wonder at these things may well be mingled with some degree of awe. For it is impossible to regard them without seeing the great and mysterious problems they put before us. What is, to our reason, this vast design, of which such myriads of separate and successive living generations are the interpreters to our senses? This question, and others collateral to it, are forced upon us almost as a necessity of thought. We see no way open to their solution. That there is a design; - that it is not accident, or a blind necessity, which evokes and maintains this world of life around us, need hardly be dwelt upon as matter of argument. The marks of law and mutual relation; - of purposes fulfilled by organisation and instincts; - are so indelibly impressed on the whole, that no seeming exceptions or anomalies can weigh for a moment against them; or leave a doubt as to the unity of plan, and its derivation from a higher source than physical science can reach. There is no neutrality in this question. To doubt the fact is to cease to reason at all.

But in recognising this supreme creative power, of which to our reason Man is the highest exponent, we are far below any comprehension of the great scheme in which we occupy this place. The question we have stated still presses upon us, as to the design of this profuse variety of life on the globe, so far beyond all reckoning of number or thought? It is easy to gather vague replies from those who look but on the surface, and indulge the belief that all these things were made for man, and his supremacy only. Such cannot be the belief of those who have read the book of nature fully 
and fairly. This great volume, while ever multiplying the proofs of power and design, inculcates a more humble interpretation of them; instructing us that we can approach the question through negatives only, which leave us far short of the solution sought for.

The most important of these negatives, undoubtedly, is that just alluded to, forbidding the notion that all other life on the earth was created for the uses of man. We should scarcely stop to argue this matter, were it not that one or two particular points of proof have an interest beyond the simple terms of the question. The first of these is the certain evidence in the long series of fossil remains, of successive periods of animal life so far anterior to man, that no calculation of ours can measure or approach them. Their various forms (from the minute Infusoria, the shells of which compose the mass of many of our existing rocks, to the huge and strange aspects of the Oolitic and Weald reptiles, and the endless other varieties which now fill our catalogues) were doubtless adapted to the several successive conditions of land, sea, and air in which they had their existence. But no reason or plausible hypothesis can bring into connection with the human race these tenants of an elder world. We may recognise the fact that the forests of those remote ages gave origin to the beds of coal, which for the last two hundred years have ministered so largely to the uses and powers of man. But we cannot equally apply this argument to the animal creations which have preceded us. No more direct relations than those of type and structure connect, as far as we can see, this ancient animal life with that of our own day. No purposes or final causes can be assumed as a necessary link between them. Any notion of tentative acts of creation must at once be put aside. To say nothing of the change it makes in our conception of the Creator of all, such notion is wholly contradicted by the actual gradation and 
intermixture of life in these successive epochs. That creatures of higher organisation are found in the later than in the earlier periods, - and highest of all, in the existing world, - is well assured as a fact. But this fact is limited to a general denotation of progress. The steps in the series are blended and broken; and Man walks the earth surrounded by beings as low in the order of life as any which the first fossiliferous rocks disclose to his view.

This argument is drawn from the ancient part of creation. We take another from that part of it nearest to man;-if not in date of time, of which we are ignorant, yet certainly in all that relates to physical organisation and intelligence. We allude here to the Anthropoid Apes; and very especially to the Gorilla and Chimpanzee, which have lately engaged the especial attention of our most eminent naturalists. These creatures, thus far approaching Man in physical features and certain intellectual functions, have no relation to him besides, save as the rare and short-lived tenants of his zoological collections; where they are gazed upon for a few months by the curious, and then pine away and die. They are few in number; inhabit very limited spaces; are seldom seen by man in their native haunts; and minister to none of his uses, directly or indirectly. They might altogether disappear from the earth without leaving behind mark or memento of the change.

An instance, thus special and significant, renders needless the many and obvious proofs which might be drawn from other parts of creation. In fact, we only touch upon this subject as a part of that great problem of life upon the earth, which is now deeply engrossing the philosopher as well as the practical naturalist, under the shape of questions far better defined than in the earlier ages of human speculation. That the great design of the Creator on our globe extends beyond, and comprises more than, the mere present exist- 
ence of Man upon it, may be fully urged without degrading the latter, or altering his place in the scale of created beings. Highest in organisation - supreme in intellect and the moral sense - single in his capacity for language - ministered to in every way needful to his bodily wants, and to his higher feelings and sense of beauty, by nature animate and inanimate around him - capable of raising himself, by his faculties of reason and imagination, far beyond the world he inhabits this is a being whose nobility cannot be degraded, or his high destiny annulled, by admitting that the earth is not a dwelling-place for him alone in the purposes of creation.

Nor is our conception of the Creator of all narrowed or impaired by this recognition. He has given existence to man. He has given life to countless other beings, inferior to man, but independent of him. To confess our utter inability to say why these things are so, is but to add another to the questions unapproachable by reason, and which it is our wisdom to recognise as such. We are sure that there is design, wise in itself and certain in its fulfillment. The doctrine of Final Causes has incurred some discredit, not from any inherent fault as a principle of reasoning - for this is unimpeachable - but from the rash and premature endeavours to carry it beyond the bounds of just induction.

We come now to another class of questions respecting animal life, more special in character; questions which have been, and yet are, the subjects of warm controversy, and strikingly illustrate the spirit of modern enquiry. We allude to those regarding the true nature and definition of species; - their capacity for change or transmutation; - the existence of types in the different grades of animal life; - the relations of date or order of succession of these several types, genera, and species; - their connection with different geological periods; - and, lastly, the manner of generation 
or reproduction, so essential a part, as we have seen, of the definition of life itself. All these questions are closely allied; yet each is so large in its objects and details, that volumes have failed to exhaust the argument. We shall take them up chiefly on points which may best show the character of the controversies to which they have given rise. That some of these controversies have been carried on with bitterness can scarcely create surprise. The new doctrines put forward have in many ways contradicted harshly the opinions held heretofore; and offence to the sober-minded adherents of these opinions has been increased by the bold and dogmatic tone which some of their opponents have assumed, and the extreme conclusions to which they have pushed their material hypothesis. Such discussions, moreover, deeply involve the relations of Man to the rest of the living creation; verge on various points of religious belief; and in these respects well explain the earnestness given to their pursuit. In what we have to say of them, we shall seek to put each topic in its simplest form, and to deal with all impartially; as questions which science has raised, and for the solution of which, if attainable at all, we must look to science alone.

First, then, among these questions comes that of the nature and permanence of Species, as distinctive of the different forms of animal life from the highest to the lowest. Our scientific readers need not be told how warmly this question has lately been agitated by naturalists. The controversy, which had its birth in France, and there provoked personal animosity as well as scientific dispute, was translated to England in a mitigated form; but has here also stirred up the depths of an argument, stretching beyond the limits of former enquiry, and liable in some part to the imputations of which we have just spoken. The laws which have governed the creation and succession of living beings come 
at once into the question; while the doctrine of transmutation of species regards man himself as a possible developement from forms lower in the scale of life.

The whole argument, in fact, concentrates itself on this point. Are Species - best denoted as such by sexual character and the power of propagating their like - to be considered fixed and immutable as they came from the hands of the Creator, or subject to such variations only as tend always to return to the original type? Or is there an inherent liability to or faculty of change, either from accident or the operation of common laws, which can, and does, in the course of time, create new species out of antecedent ones; an extension in effect, and higher result, of that very principle of change by which varieties and races are brought into existence? The great name of Cuvier appears in the foreground on behalf of the former opinion: Geoffroy St. Hilaire made himself the chief of the opposite party. In England, the transmutation doctrine first gained currency through that well-known work the 'Vestiges of Creation;' and has since been espoused by other writers of great and merited reputation. Many of our most eminent naturalists, our geologists especially, have entered with earnestness into a controversy, rendered inevitable from the progress of their science and the new phenomena ever coming before them. Looking generally on the conflict as it now stands in this country, we still find a predominance of opinion for the fixity and permanence of species under the definition we have given. But at the same time we notice a certain cautious reserve in announcing any absolute or final opinion on the subject; an effect doubtless of the ambiguities which still surround it, and the difficulty on each side of reaching any other than presumptive proof. While approaching the question ourselves in this spirit, we shall seek to frame our argument as a vindication of the older belief; feeling that 
this cause is one not to be lightly given up to the demands of a new doctrine, still unproved in some of its most essential parts.

The onus probandi, indeed, unquestionably rests here with those who believe that species can undergo such transmutation, as permanently to change the conditions upon which this distinction has naturally, or even necessarily, been founded. It is their business to show some unequivocal instances of perfect transmutation; or, in default of this, some such approach to it, by gradations manifestly progressive, as to warrant the presumption that time only is wanting to complete the change. Less than this cannot be received in evidence of fact, however plausible an hypothesis of possibilities may be made to appear. The limit-line drawn around each species, by its power of self-reproduction, may not be broken through without more complete proof than any yet proffered to us. No unequivocal instance has hitherto been obtained from any part of the animal kingdom to satisfy fully these conditions. The question therefore remains one of possibility and presumption only. Possibility cannot be denied; but the advocate for the permanence of species, resting upon much that is assured to his knowledge, has a right to ask that the opposite doctrine should be fairly fortified by fact before its admission even as one of the outworks of science.

The arguments for the hypothesis of transmutation are, mainly, the variations which species actually undergo; and which in many cases, especially where man is the artificer of new breeds, become fixed and hereditary; - the fact that in a certain number of instances the intermixture of species is prolific; - the existence of certain archetypes or general forms, upon which the many specific forms are founded; - and the tendency of all research, in the fossil as well as living world, to bring the gradations between these forms into closer con- 
tact; filling up, more or less, thereby the void places in the series of genera and species before known.

Other arguments there are, but these lie at the root of the question, and may be taken instar omnium. In pursuing the controversy, one party has found it needful to assume, and the other to allow, an unlimited license as to time. We do not go over the geological proofs as to this matter, now become so familiar to all. It is enough to repeat, that prior to man and all the creatures occupying the world with him, there have existed on the earth successive and separate conditions of animal and vegetable life, as faithfully recorded in the rocky cemeteries below us, as if they were the creation and destruction of our own day. Though the order of succession is distinct here, no human estimate can reach the period of time these successions involve; so vast is it, and so broken by intermediate changes to which no measure can apply. In one sense then, that of the existence of life on the earth, time has no numerical limit which we can assign. But the advocate for transmutation of species must take it, subject to a question as to the nature of these intermediate changes or catastrophes. If they be such as to close one epoch of life on the globe before the creation or commencement of another, then the argument, as far as time is concerned, must be limited to that latest epoch in which we are now placed. Many of the gaps in the structural scale have been filled up, indeed, from the fossil remains of former periods; but until some series can be shown connecting these periods together without breach of continuity, the hypothesis of developement or transmutation cannot fairly borrow time from these anterior epochs, for the changes it presumes.

Nor does it really lose much by this limitation. The fossil remains of former ages of life afford no evidence as to transmutation of species, which may not as plausibly be drawn from the existing animal creation. We find the same general 
types of structure, declaring to our reason the unity of the creating cause, but evolved under many and singular diversities of form; - with the special fact superadded that each epoch contains species peculiar to itself. Different species more or less approximate to each other; but in none of these periods have we yet found any such series as to indicate an actual passage from one to another; or anything more than this proximity, itself expressed in all cases by the same special forms and relations of parts.

There is, however, one point of connection beween these periods, which bears in some sort on the question before us. We allude to the fact - indisputable in itself, and deeply interesting in all ways - of the successive appearance of higher types of organisation and conditions of existence, in rising from the earliest of these epochs to that in which we have our own being. We shall revert to this topic hereafter, and notice it here only to show that it does not sanction, or even favour, the doctrine of transmutation. There is no regular gradation either as to time or type, such as the theory would require. New and higher forms of life come in with new epochs, and continue to be associated with the lower types that before existed. Recent research has carried back some of these higher forms into geological ages more remote than was once supposed their limit; and this may go yet further. But it would not then, more than it does now, affect the argument we are holding.

The existence of such common types or plans of structure, extending throughout the whole domain of life and giving foundation to all special forms and varieties, may seem at first sight to furnish some valid argument for transmutation; and the more so since these types are found in one sense to graduate into one another. The individuals of higher type throughout the several great groups of the animal kingdom, pass through certain stages of those lower in the scale, 
even as far down as the simple germ of the Protozoa, before reaching their final and special organisation. Of this very curious fact the human structure itself is an example; a fact not neglected by those (and, strange to say, from paradox or petulance there are such) who love to degrade man in the scale of being. The attempt is a futile one. The Protozoon stops at his destined place in the lowest scale of being. Man reaches by definite steps the high organisation which is designed for him. Each of these, and every species intermediate in the animal world, attains, and is arrested at, the point marked out for it in the long line of created life.

The whole subject of types is one of the deepest interest. The doctrines of St. Hilaire led him to refer all animal life to a single primitive type only. Cuvier, followed by the far greater number of naturalists, has denoted four as absolutely marked and distinguished in nature. Subordinately again, or included within this theory of types, comes the more recent doctrine of Homologues; teaching us the relations of equivalent parts of structure throughout the animal world. A fine conception of Goethe - half poetry, half philosophy - became, under what we will not call the sober enquiry of Oken (for the genius of Oken has no mark of sobriety upon it), but became by his research and that of other naturalists, an integral part of natural science. Had we space for it, we should gladly put before our readers some account of the valuable contributions of Professor Owen to this curious branch of knowledge; - the researches by which he has confirmed the view of the Skull as an extension of the vertebral column; - and his remarkable work on Limbs, in which portions of structure in different animals, seemingly the most unlike in aspect and use, are all resolved into common relations of typical structure. These things must be regarded not as mere naked facts, but as the interpreters to our reason of an Almighty design, in action from the earliest ages 
recorded in the rocks below us; of which Man himself, highest and foremost in the series of types, appears to our present view as the consummation. But we have no right, on this latter point, to dogmatise as to that futurity of time and change which goes so far beyond all human knowledge or comprehension.

From the system of types, however, the advocate for transmutation of species can draw no fresh argument for his doctrine. It is still a series, more or less complete in its parts, of which each member or species, in past as well as present periods, has its defined and specific characters; and continues to have them as long as its existence comes within our view. The type, whether general or particular, represents to us certain common outlines (ideas we are tempted to call them) in the scheme of creation, to which these separate members belong as individual parts. Were there anything like actual transmutation amongst them, we might expect to have our catalogues crowded with instances of this in every stage and aspect of its progress, or even to have evidence of it under our own eyes.

This brings us to what is a main argument in the matter; viz., the variations which actually occur in living species, and which strongly tend to become hereditary, if the causes of variation be continued. Examples to this effect are so numerous and familiar that it is needless to cite them in detail. They occur most strikingly among animals domesticated by man, or artificially bred for his uses or pleasures. But they are produced also by variations of climate, food, and other physical conditions tending to perpetuate certain forms at the expense of others; - sometimes also by those more mysterious influences of generation, of which we can say little more than that they exist; and, existing, must be the source of numerous facts and anomalies, to which neither reason nor research furnishes any other clue. 
In Man himself these varieties take their highest, and perhaps most heterogeneous character. They are testified especially in the difference of races; a distinction so strongly marked in particular cases, as to have led some naturalists - erroneously, as we believe - to refer it to an actual diversity of species. That strangely familiar friend of man, the Dog, produces varieties scarcely less numerous and remarkable. An eminent French naturalist has stated that if we were to reckon as species the different breeds of this animal, we must carry the account above fifty. The extent to which such deviations may proceed and become hereditary in a race, is one of the most curious enquiries in natural history; as well in reference to the authentication of true species, as in connection with the subject of Animal Instincts, to which it is closely related. We believe that there is a natural limit to change in each particular case; and think it probable that the deviation, though differing under different conditions of animal life, has already reached its maximum in the animals bred and domesticated by man.

Even here, then, the advocate for transmutation fails to make good his case; since it may almost be affirmed that the particular capacity for variations in each species forms in itself a specific character. These varieties or deviations are not changes of species, but changes within them; and, with few and ambiguous exceptions, are confined to certain limits which the law of reproduction of species strictly defines. This law, in truth, comprises all the cardinal points of the question. It is a natural definition put before us, and so strongly marked, that the argument as to design might well be made to rest on this alone. If an instance could be brought of the intermixture of two species in generation producing a fertile offspring, capable of breeding with similar hybrids, or otherwise perpetuating the physical changes induced, the law would doubtless be impugned in its 
generality; and the possibility might be urged of numerous such instances, if time and chances are taken into account. But we doubt much whether, in the animal kingdom at least, an unequivocal case of this kind really exists. It may be admitted that several hybrid species (the equine, canine, ovine, and, possibly, but less certainly, some other animals brought immediately under human culture) are to a certain extent prolific.* But the true hybrid does not propagate with the hybrid; and its power of propagation, even with the perfect species, is very limited in degree, and soon comes to an end. On the other hand, we believe there to be no recognised instance of absolute infertility in two varieties of the same stock, however wide their deviation from each other. This argument, then, for transmutation halts on the very threshold; and, duly examined, may even be appropriated for the opposite conclusion.

The sexual relations cannot be omitted as a point in this question. Weighing fairly all the circumstances - and, amongst others, the period of gestation (different even in the cognate species of the dog and wolf, though alike in the dog and jackal) - it appears almost certain that no real or permanent change of species can take place without a concurrent mutual adaptation of the two sexes in its progress; - a contingency so improbable, seeing all that is required, that we cannot but regard this as one of the most cogent objections to the doctrine before us, and meriting more attention than it has usually received.

A word or two more must be said in regard to the varieties in species themselves. Strictly speaking, these are not so much structural differences, as variations in size, configuration,

* We are bound to notice here the experiments of M. Rouy of Angoulême, who succeeded, by the inter-breeding of hares and rabbits, in producing a new hybrid race, fertile, as he alleges, without reversion to the original stock. Further research is required to attest the duration of this fertility. 
integuments, colour, and other external characters, subject in each case to limits which they cannot transcend. The despotic folly of a Prussian monarch might breed, as well as steal, gigantic soldiers for his guards; but could not change in a single particular the anatomical characters of the men thus forced into his ranks. We have before referred to the Dog. With the exception of a slight change in the bones of the hind foot in some breeds (the maximum of variation, as far as we yet know) the bony structure and internal organs are the same under all its numerous varieties. The teeth, now so important a diagnostic mark, are alike in all. Its animal instincts, though modified, or often suppressed, by human culture, are essentially the same throughout; and the dog himself well knows his own species, whatever varieties it assumes. It is needless to cite other instances, as they all correspond in their bearing on the question before us.

A point upon which stress has been laid by the disciples of Lamarck is the close approximation of the Anthropoid Apes to man; warranting, according to them, the notion that the lower may here have passed into the higher grade of being. Admitting the likeness to its fullest extent (the Simia quam similis! of the old Latin poet), it is still but the mark of closest proximity in the scale. The evidence, either anatomical or of other kind, as we have already mentioned in speaking of these creatures, goes not a step further. And against the transmutation hypothesis here, we have the fact stated by Owen, that certain of the osteological differences between these animals and man are so characterised as to be insusceptible, from any known external causes, of the changes required to accomplish such transmutation.

The arguments we have now been using for the permanence of species will be familiar to many of our readers. But there are others to whom the question has come only in a crude and general way; and to these it is well, seeing how deeply 
this enquiry strikes its roots into the mysteries of creation, that it should be presented in such form, as to make clear the distinction between what is speculation only, and what a sound induction from the facts which science has disclosed. We may fairly use the word vague here, as descriptive of the manner in which the doctrine of transmutation is propounded to us. There has been no distinct understanding as to the foundation, or first steps, of the scheme supposed. With some it is a notion of gross materialism altogether. Genera and species of living beings come into existence through undefined combinations of matter, and are mutable without limit from material causes acting upon them;-or, as some prefer to phrase it, from a nisus of the animal itself to obtain new scope and powers of existence. Others, more modest in their assumptions, have supposed a few original created forms, capable of gradual developement into new species, unlimited in variety, if unlimited time be given to work the transformation. We cannot reasonably require any actual date for the time thus demanded. But we may fairly complain of the vague asseveration on which the whole scheme is built. We have a right to ask for some denotation, however general, of these primitive beings, the parent stems of the genera and species we now see around us. The doctrine of types, as already explained, furnishes no reply here; nor to the questions we have a further right to ask, whether any of the primitive forms yet remain in existence as such? Or whether the principle of change is now exhausted in power, and the existing genera and species represent a scheme of transmutation worked out to its end? Other questions equally press for answer, showing how unstable the hypothesis is in its first principles; and how little fitted, upon present proof, to meet the demands of a sound inductive philosophy.

In arguments of this nature, it is of great value to obtain 
such instances as are not only indisputable in themselves, but extend their conclusions to other kindred cases. The Electrical Fishes appear to us to furnish a striking example of this kind. From what processes of transmutation can be derived the several forms of bodily apparatus in these animals, capable of generating this singular power, and putting it into action by their will? The argument is analogous as relates to the Poisonous animals, whether reptile or insect in kind. The differences of the poisons themselves, and of the parts instrumental to them, are such as to annul all idea of primitive community of species, even in the case of the venomous serpents, where it would be most natural to expect it. The same reasoning might be applied to the Luminiferous animals; and to many other cases, where some special property or provision pervades many species, yet leaves distinct and lasting the individuality of each.

We might find a further argument in the different, yet definite figure and size of the blood-corpuscles in different animals; - a very curious subject, but not yet enough explored to furnish any certain inferences. The reasons we have stated, however, are presumably sufficient to justify the belief, not indeed in all that have been denominated Species (for doubtless many mere varieties and duplicates are catalogued as such), but in the fixity and permanence of the majority so recorded. No sound reasoner will raise an objection from the multitude thus supposed distinct in their origin. Whether we look to the great or small in creation; - whether to the stars of heaven, or to the infusorial animalcules of our own globe;- - equally must we regard number in the hands of the Creator as a thing wholly apart from our own feeble and limited conception of it. The miracle to us is the act of creation itself. This recognised, (and can it be denied?) the exertion of the power is subject to no artificial limit of ours. A hundred and a hundred 
thousand are the same to all actual or possible comprehension of the matter.

We have already spoken of the manifest design in the vast and various profusion of life spread over the earth at successive epochs; and we may now advert to another case, where designed progression is obvious to our reason, though in a different sense from that of the progressive transmutation of species. This is the fact - already adverted to, and well authenticated - of the successive introduction of higher forms and attributes of life into the series, as time has moved onwards through the ages anterior to our own being on the earth. From the period when the Cephalopoda were supreme in the animal kingdom, to that when Man became its head, we have a series of types, each rising in organisation, of which Fishes, Reptiles, Birds, and Mammals represent the most remarkable forms. The only controversy as to this point has arisen from certain seeming irregularities in the order of succession; these higher grades of life coming in without any apparent conformity to our measures of time or relative change. But the main fact is in no way impeached by this irregularity, and intention is on the very face of it. Making every allowance for our inability in many cases to say which of two proximate organisations is highest or most perfect, we cannot doubt as to the relative character of the fossils of the Silurian and Devonian ages, and that of the Oolitic remains, where the Mammalia first come into view; - nor, again, can we hesitate as to the relation of Oolitic life to that of our own day.

We may quit this subject with the general remark, that if transformation of species be ever proved, it will probably be so in the lowest forms of animal life, where the organisation is of the simplest kind, and where the functions seem limited to mere maintenance and reproduction; - the latter, moreover, effected in some of them by means very different from the 
analogies of higher animal life, and more akin to the characters of the vegetable world. Even here no actual transmutation has yet been made known; and the argument we have been stating remains therefore still untouched. But we are bound to add, that much and able research is now in progress, bearing on this question of the true definition and limitation of species; and followed chiefly through the phenomena of breeding and hybrids, as the best and most legitimate mode of approach to its solution.

All these topics have close mutual kindred; and we have taken advantage of this, to bring into the foregoing argument many collateral topics illustrating the vocation and spirit of modern science. We now come to another question, linked into the same chain, viz., that of spontaneous or equivocal generation; - the enquiry whether new species are still brought into existence, or have been so, within what may be called the human period of life on earth? We know that certain species have utterly passed away within this period; and that others are in assured progress towards extinction. Man himself has been largely concerned as the agent in these changes, whether of diminution or annihilation; but physical conditions have doubtless also had effect. Is there anything in the nature of an equivalent to them, by new acts of creation, or by the spontaneous production of fresh forms of life?

Here again we are without a single fact in absolute proof. The extinction of certain species (and these, as far as we know, exclusively of the higher animal orders,) does in no way imply the creation of others; and the only affirmative presumptions that can be offered are drawn from those minute and obscure forms of life, where the sexual distinctions are little marked, and the functions of reproduction of unwonted kind. Accordingly, we find that those who hold this belief dwell much upon the Entozoa; and yet more on the appearance 
of animalcule life in various artificial compounds of organic matter, under forms peculiar to each. The latter fact is as incontestable as it is curious. But the conclusion from it must ever be a doubtful one; seeing what we know of the wonderful retentiveness of reproductive power in the ova or germs of such animalcules. We may readily conceive, and we actually know, the continual presence of these invisible elements of life in the earth, air, and water around us; ready to start into form the moment the physical conditions are present which can give them their proper habitat and nutriment. Many analogies in the vegetable world familiar to naturalists favour this view; and none, as far as we know, contradict it. The argument for spontaneous generation from the Entozoa is refuted by the fact that, with the exception of a few species which propagate by budding, they have all male and female organs. The Polygastric Infusoria have been brought in to support the doctrine. But in all the varieties of these animalcules there are fixed and invariable forms; and these, it may be added, closely assimilating them to the fossil infusoria, which existed ages ago.

Thus far, then, the opinion rests on very slender authority. We may add one argument, not usually quoted, yet as valid as any of the foregoing; viz., those new forms of epidemic pestilence, which from time to time have appeared in the world, devastating whole continents in their progress, and depending, as we are disposed to believe, on organic and living matters diffusing themselves as the virus of disease. In no other way, as far as we can see, are these wandering pestilences to be accounted for, than as derived from a materies morbi, capable of reproducing itself, and therefore coming strictly under the character of life. But here again, while admitting this view, we cannot affirm that the germs may not have existed for ages before, awaiting develope- 
ment; and the argument therefore is as loose in proof as all others which bear upon this question.

We pass here by a short step to another topic, one of the most interesting, but most obscure, in natural history, - that of the reproduction of life. Modern science, active as we have seen in its interrogation of all nature, has eagerly explored this subject, and obtained many new facts and conclusions; but none which give a key to the ultimate mystery of life propagating similar life. The steps made are all intermediate; in no respect are they final or complete. We may refer, for example, to the recent discovery (due to the microscope) of the cell structure, as the first distinct developement of individual life, and the rudiment of future growth both in the animal and vegetable world. In the zeal with which physiologists have adopted and pursued this discovery, there has been somewhat too high an estimate of its real value. The fact, indeed, is curious and unexpected; but it carries us onwards by a single step only. Cells themselves, with whatever nuclei they may contain, must be derived from some more primitive germ or aggregation of matter; and when we read of cell force and cell growth, we have reason to ask what these terms really convey to us. It is even easier to conceive of growth from minute vascular structure, than from cellular aggregation: but both conceptions leave untouched the great problem of generation; the assumption of infinitely different but perfectly definite forms, from rudiments thus simple and seemingly similar. We feel that there is something beyond which no hypothesis, however bold, can cope with; - that we are yet far from reaching to that mysterious principle or power, by which the life of individuals and species is elaborated and maintained, generation after generation, under every grade of likeness or diversity, each equally inexplicable!

Modern research into these phenomena has not been 
limited to the discovery of formation through cells. Other strange facts and seeming anomalies, in some part indicated by earlier naturalists, have been subjected to more exact enquiry; and, had we room for it, we might state many most curious results, particularly as regards those phenomena which the researches of Steenstrup and others have disclosed to us. The Greek, 'that musical and prolific language of ancient philosophy,' has been drawn upon so largely for scientific purposes in our own time, that we cannot quarrel with such terms as Metagenesis, Parthenogenesis and Agamogenesis, though somewhat ostentatious as applied to the most minute objects in creation. They serve to betoken what are indeed very strange and complex modes of reproduction; in which the sexual influence (though not lost, and in some part and form always necessary) is in certain cases so wonderfully concentrated - concreted we may express it - in the organisation, that a dozen generations may be evolved in succession without any renewal of the male influence in reproduction. This fact has been amply established by experiments reaching as far back as the days of Reaumur and Bonnet, and is well exemplified in the instance of the Aphides; the diversities of which - viviparous or oviparous, winged or wingless, alternating or without obvious rule of succession - offer a multitude of problems to sober, as well as to speculative thought. This budding forth of a germ principle through successive generations from a first single fertilised germ, while closely connected with the principle of animal metamorphosis, is the fact which, more than any other, forms the. link (very difficult indeed to dissever) between animal and vegetable life. The Entozoa, Polypi, Medusæ, \&c., all enter into and illustrate this great natural relation. The phenomena of fissiparous generation variously and strikingly attest it:- those curious cases where entire and repeated division of the animal does, under certain limitations, repro- 
duce the perfect form in each of the divided parts. In some of the Infusoria, the problem is further perplexed by a double manner of generation through ova, and by self-division of the animalcules themselves.

All these things, and others equally wonderful - such as the modes of parasitic or complemental generation described by Mr. Darwin in his Monograph on Barnacles - may well astonish those who come unprepared to the subject. In reality, however, there is nothing more unintelligible here than in the familiar facts of ordinary generation, nor more wonderful than what we before knew as to the economy of other animals higher in the scale of creation. The modes of reproduction of the Bee (especially as last developed by the researches of Dzierzon and Van Siebold) may be taken as an epitome of all that is most marvellous in this great function of life. The true mystery, as we have already said, lies deeper; and is equally associated with every variety and aspect of these phenomena. It is one of the many cases in science and philosophy where familiarity gives a semblance of knowledge; satisfying the shallow enquirer, but otherwise estimated by the more cautious seeker after truth. The several questions we have been discussing, and others not less difficult of solution, are all subordinate to the single problem of the transmission of life and likeness from one living being to another. All theories and systems are alike arrested at this point. The problem, in truth, is one insoluble by the present capacities of man.

Another topic of eminent importance to all our views of life, and the economy of living beings, is that of Animal Instincts. Much has been observed, thought, and written on this subject; but less connectedly, we think, than its interest requires. Facts have been multiplied and better defined; and the.special structures serving to the fulfillment of instincts more carefully, yet for the most part vainly 
explored. For the great problem here remains as entirely unresolved as in the earliest days of ancient philosophy. What is the source or proximate cause of those actions - definite, peculiar, and permanent in each species - which we call instinctive, as distinguished from the acts of reason and intelligence? The main points of doubt, speculation, and controversy are all concentrated within this question. It involves one which in some sort is precursory to all, viz., the reality and nature of the distinction between reason and instinct; faculties so closely bordering on each other, and often so blended in the same acts, that it becomes difficult to distinguish or dissever them. To obtain a just definition, we must look at the more simple and extreme cases of each. 'The absolute hereditary nature of Instincts; - their instant or speedy perfection prior to all experience or memory;their provision for the future without prescience of it; - the preciseness of their objects, extent, and limitation;- and the distinctness and permanence of their character for each species,' are the more general facts upon which we define true instincts, and contradistinguish them from the acts of mind and reason. These two great faculties may be said to exist in inverse ratio to each other throughout the whole scale of animal life. Where intelligence is highest in power and effect, instinct is lowest and least in amount. It augments progressively as we descend in the series; and at some point, hardly to be defined, seemingly embraces and gives origin to all the acts of animal existence.

The only probable advancement, as far as we can see, in the theory of Instincts, will be through such researches as may determine their more exact relation to reason in the same individuals or species. The very blending of these faculties in the higher order of animals, while it perplexes in some points, does in others offer the chance, if not the certainty, of illustration to both. Without undermining the 
distinction between them by metaphysical subtleties, we may well admit that the questions they suggest are in great measure the same in kind, and of like difficulty in solution. The method of research we suggest through these common relations, though often touched upon in part, has never been systematically pursued. It would require varied experiments, as well as minute observation. It must of necessity be an assiduous labour, and divided among many; but also a labour of high interest, and aided by numberless facts already ascertained, but not yet collated or reduced to order. A systematic work on Instincts, derived from every province of animal life, and carefully brought into relation with those various degrees of reason which animals possess, would (even if but partially completed) be of high value to physiological science in its every part.

Associated, though less closely, with the foregoing topics, comes another enquiry which has earnestly engaged the naturalists of our day; viz., the manner of distribution of the types, genera, and species of animal life over the surface of the globe. The diversities of such distribution have long been noticed; but to botanists, and especially to Decandolle, we owe the first clear conception of geographical provinces, within which are located certain predominant typical forms, diffusing themselves as from a centre ;-arrested in some cases by the intervention of sea or land; - in other cases mingling: on the border with the types and characters of other provinces. In animal life also we find this local distribution, with conterminous mingling and affinity of genera and species, strongly marked; and though the boundaries of such provinces are still not fully defined, we are sufficiently assured of the fact to reason upon it as a part of the living economy of the world. And a most curious fact it is; depending, as it must do, either on the original conditions of animal creation, or on the great revolutions of the earth's 
surface, recorded by geology; - those mighty interchanges of land and ocean, by which continents have been raised or submerged, climates changed, and all living nature brought into subjection to this elemental strife.

Australia is generally quoted as the most striking example of such local limitation of forms, both in animal and vegetable life. This strange Continent - scarcely known a century ago, now the flourishing seat of British empire in the Southern hemisphere - stood, when discovered, in a sort of solitary contradiction to the rest of the known world. Of 4,100 species, forming its earliest recorded Flora, only 106 were found elsewhere. The Eucalyptus and leafless Acacias, in their numerous species, inflicted a sombre hue on its scenery, while the marsupial animals, with other strange configurations of animal life, gave an eccentric and paradoxical character to the Fauna of this region. The Galapagos Isles, so well described by Darwin, furnish another example even more striking from its limitation. These isles (volcanic in origin), though but 600 miles from the South American coast, yet form a distinct province both in their Flora and Fauna; with scarcely a single organic production which is not aboriginal in species, and unknown elsewhere. South America itself is the peculiar domicile of the very singular order of Edentata, or toothless animals, which are here found both living and fossil; while north of Mexico they are unknown except in the fossil state. The Sloth, that strange and grotesque member of this order, and the Armadillos, are found in America only. New Guinea, with some island groups to the east, forms a particular zoological province; singular from being destitute, with one exception, of all warm-blooded quadrupeds. The Elephant, Rhinoceros, Giraffe, Hippopotamus, \&c., are limited now to certain portions of the old world, though their fossil remains are much more widely diffused. The marine animals, much more vaguely of course, 
give evidence to the same fact of local limitation; and even lake and river fishes demonstrate it, comparatively narrow though the spaces are which they occupy. We may seem to see reason why the Salmon, found in all countries bordering round the Arctic Circle, should nowhere exist in the Southern hemisphere. But how are we to explain the different families of fish, found by Agassiz in each of the great fresh-water lakes of North America, connected as they are by a common river? Or how the fishes peculiar to the Ohio and many other rivers? Or the species limited in existence to some of our own rivers and lakes?

Such instances, which might be endlessly multiplied, show how curious are the problems belonging to this part of natural history; and how perplexed in every part by the doubt of what may belong to a primitive geographical distribution of created beings; - what to the revolutions of the surface of the globe, paroxysmal or gradual, which have since intervened. The argument for the former, supported as it is by the complete analogy of vegetable life, is too strong not to compel belief; though leaving it doubtful to what extent the limitations of localities and species originally existed. Further research may do something towards clearing away these doubts, but can never wholly remove them. The unquestionable changes in climate and other physical conditions essential to life, from geological revolutions on the earth's surface; and the mighty influence of Man when he became a tenant of the globe, in multiplying, destroying, or transplanting whatever of the living creation existed around him, have removed many of the marks or outlines which might have denoted this primitive distribution. Fossil geology to a certain extent comes in aid of the research ; though in solving some questions it evokes others not less difficult. In the vast periods of time through which it carries us, we see the same revolutions of surface, elevations, depressions, and 
changes of land and sea; but the farther we recede from our own time into these depths of ages, the more entirely do we lose all analogies of geographical distribution. 'Even in some of our most recent strata,' as Professor Owen remarks, ' fossils occur for which we must seek the representatives in America; and to match the mammalian remains from Oolite, we must bring specimens from the Antipodes.'

In treating of these various questions which have relation to Life as the subject of modern science, we have only partially alluded to the enquiry denoted by the special term of Animal Physiology; - the history of those organs and functions through which vitality receives and maintains its individual existence. This subject, in truth, is too vast in outline as well as details, and the discussions it embraces too various and important, to be dealt with in any single Article, even exclusively thus directed. The functions of nutrition and assimilation, - of circulation and respiration, - of secretion and excretion,- and of the nervous system in its several parts, - all these have been the objects of refined experiment and sedulous observation by the physiologists and physicians of our day; and with results which give a new face and form to this branch of science. But while putting aside the subject at large, there is one class of the functions just named which we cannot wholly omit when treating of physical science in its relation to vital phenomena. We mean those wonderful functions which are fulfilled through the instrumentality of the nervous system, and which we cannot err in describing as of far higher interest than any others of the animal economy; seeing that they connect the conscious being, whatever its grade in creation, with every part of its own organisation and with the world without. Sensations in all their forms, volitions in all their acts, find transmission solely through this portion of structure; - one so little intelligible to the eye or outward 
observation, that not a single anatomist or philosopher of antiquity placed a right interpretation on its nature and uses. Modern science has encountered the subject with the better appliances of experimental enquiry; and though much remains to be done, and much more may be deemed wholly unattainable, yet we can safely affirm that some of its greatest achievements are to be found in the anatomy and physiology of the nervous system.

Into the details of these discoveries we cannot enter. They relate chiefly to that organisation and distribution of nervous matter (including the Brain as its highest developement) through which this power is generated and transmitted in fulfillment of the various functions of life. They include yet further the especial relation between the several parts of the nervous system and their different functions; whether such as appertain to animal life appropriately, or those more purely of organic kind. This latter distinction in itself may be deemed a recent discovery, and one prolific in curious and instructive results. So, in truth, are all those discoveries which connect particular parts of the nervous system with the offices they fulfill; from the highest and most complex forms of this structure in man, to the bare manifestation of it in the lowest grades of animal life. Every step in these researches opens out new views to the speculative eye, and offers new problems for experiment and reason to resolve. The successive and successful labours of Bichat, Bell, Magendie, Marshall Hall, and other physiologists, thus directed, have been more recently extended by those of Brown Sequard, to whom we owe many interesting facts in this part of animal physiology.

Among these various topics, there is one question so closely allied to some we have been discussing, that it cannot fitly be put aside. Is there any special physical agent, acting in and through the nervous system, and by such action giving 
fulfillment to its numerous offices in the living economy? Or must we look to some mysterious power existing here, apart from matter and the forces acting on matter, as needful to explain the phenomena, and particularly those which connect the nervous system with consciousness and the mental functions? This question, like the analogous one as to a Vital Principle, presses upon us almost as a necessity of thought. As in so many other cases, language has sought to evade the difficulty of solution by phrases more or less convenient for use, but which indicate no new or real knowledge acquired. We employ the terms of nervous power, principle, energy and element, - nerve-force, innervation, \&c., all preferable, doubtless, to the older phrase of nervous fuid; but preferable simply because less definite in their assumption of meaning.

To the question stated above, science has yet rendered no certain answer; but there are several presumptions favouring the view that some physical agent - analogous to, if not the same as, the natural forces of which we have so often spoken - does directly minister to the functions of the nervous system. One of these presumptions is founded on that conception of quantity, which is forced upon us in every consideration of nervous power, and is expressed equally by excess or deficiency in amount. We exhaust ênergy by action; we augment it again by time and rest. Scarcely can we name a function of life which does not include the fact of a power inherent in it, thus varying in degree. Whether we can apply the term intensity, as separate from quantity, is more doubtful; for though the distinction is valid as applied to electrical action, it is not equally so as regards nervous power. A much more cogent presumption to this effect is that furnished by time as an element in the action through nerves. This very interesting fact of a rate of motion, already conjectured and vaguely estimated, has been recently 
attested by the beautiful experiments of M. Helmholz on the crural nerves of the frog, which show that a space of somewhat more than eighty feet is passed through in a second of time. To subsequent observations of M. Helmholz we owe the further remarkable facts that the rate of motion of the nervous power in Man is more than double that observed in the frog; and that it sensibly augments with any augmentation of animal temperature. These experiments are so delicate in apparatus and manipulation, that few can undertake them; but their principle is one which in skillful hands may hereafter illustrate some of those variations and anomalies of nervous power, which at present perplex all our reasoning. Meanwhile the fact ascertained of the propagation of power in definite time, brings us to the conception of a physical force, like to those which act on matter through its molecular structure elsewhere in the natural world. And this presumption is strengthened when we consider the actual relation of these forces, and of electricity especially, to the functions and phenomena of the nervous system.

At this point, however, a serious doubt suggests itself. Can these functions, so diverse in nature and quality as well as degree, be due to any single agent of motion and power? Can we possibly predicate unity of any proximate cause, in actions which combine the functions of the several senses;voluntary and involuntary muscular contractions ; - the nervous influence directed to the various secreting organs; and the sympathies between different organs, which John Hunter well describes as the 'internuncial office' of the nervous system? This question will be directly seen as one of great, perhaps insuperable difficulty. As we cannot multiply agents to meet the many conditions just stated, or find adequate explanation of them in any structural differences of the conducting nerves, we can only approach a solution by looking to the diversities of organisation upon which the 
nervous force acts; and by presuming, as indeed we are compelled to do, that these diversities are often of a nature to evade the most subtle research. The chemist and the microscope have disclosed to us many marvellous secrets of molecular aggregation; but they have rarely, if ever, been able to tell us of that ultimate structure, which at once defines and fulfills the various functions of life.

We have spoken of Electricity as the physical power most nearly allied, so far as we yet know, to that acting through the nervous system. We are not propounding here one of the many vague hypotheses to which electricity, from its striking and complex phenomena, has given birth; but what is a legitimate inference from the most exact and delicate experiments. These, while leaving the fact of identity still unproved, and many collateral questions yet unresolved, have nevertheless disclosed such analogies and intimate relations, as to make it probable that the forces in question are at least mutually convertible, in the sense we have already given to this phrase. Had we space for it, we might relate some of those wonderful results derived from the experiments of Du Bois Raymond and Matteucci, which especially favour this interpretation. We may mention, as perhaps more cogent in its conclusions than any other, one we have ourselves often seen; where a sudden and forcible contraction, by will, of the muscles of the forearm, evolves a current of electricity capable of passing through two or three miles of a helix coil, and creating power enough to deflect the needle of a delicate galvanometer, $50^{\circ}$ or $60^{\circ}$ or $70^{\circ}$, according to the vigour of the muscular contraction. The inference here seems direct and decisive; and it corresponds with other conclusions from the experiments of Du Bois Raymond, as to the uniform direction of the electrical currents pervading all muscular fibres. Yet we are still short of that certainty which science is rigid in requiring. We have reason to 
believe all muscular action - perhaps every vital action to be attended with some chemical change in the parts concerned; and every chemical change, as we know, produces disturbance of the electrical equilibrium. Changes of temperature moreover, or molecular motions, each belonging to muscular contraction, may be concerned in evolving these electrical currents. But whatever the ambiguities of the question, it is obvious that they all lie within that single circle which comprehends and connects the great Co-related Physical Forees of the universe; - a magical circle we may well call it, as comprising within itself some of the most profound and mysterious problems which human reason can venture to approach.

We must here come to a close; although there are still numerous topics we might bring under consideration, illustrating the efforts and results of modern science in relation to this great subject of Life on the earth. It will have been noticed how often the question of Final Causes comes before us, as a consequence, and even integral part, of these enquiries. We have already alluded to this point; but cannot conclude without reverting once again to a principle of reasoning which it is of signal importance should be rightly appreciated in the interpretations it affords. A misplaced sophistry, fortifying itself by a single phrase of Lord Bacon's of doubtful meaning, has sought to impugn this method and the conclusions thence derived. It cannot be done. Such reasoning is an integral necessity of our mental constitution. The fallacy lies here, as so often elsewhere, in imputing to the use of the faculty what belongs to its abuse; since, if using that caution which the nature of the subject inculcates, we may safely and profitably employ it as a guide in research, as well as an exponent of discovery, in every part of the great domain of created life. 


\section{POSTSCRIPT.}

The foregoing article was published a year before the appearance of Mr. Darwin's remarkable work on the 'Origin of Species.' Whatever be thought of some of the conclusions at which Mr. Darwin has arrived, the value of his work as a contribution to the Natural History sciences, and as a guide to their future successful pursuit, cannot be too highly estimated. Nor is it possible to speak too strongly of the candid spirit manifest throughout this volume; leading its author to anticipate objections and acknowledge all diffculties; and to state, even perhaps beyond his own belief in them, the conclusions to which his doctrine might be liable, if carried to its extreme inferences.

Thinking it well that the argument on the other side should be fully and fairly weighed, I have made only a few very slight alterations in the article now reprinted; one of these referring to the changes in the animal world due to the principle of natural selection, operating amidst the general struggle for existence. This principle, which Mr. Darwin has so largely and effectively used in his researches, may be considered in great degree a new path of enquiry; and one which steadily pursued by exact and patient observation, cannot fail of conducting to important results. It is certain that at some future time a revision and reform will be required of all existing catalogues of genera and species. How far the needful curtailment may carry us is yet doubtful; but there is reason to presume that it will stop very far short of anything like unity of origin, even with unlimited concession of time for the process and progress of change. An original act of creation, in time and under 
design, being assumed in every hypothesis, the conception of any primordial unity, capable of evolving and multiplying itself into all the actual forms of life, is infinitely more difficult than that of many distinct primitive forms or types, brought into simultaneous or successive existence by one designing and creating Power. Numbers in truth (and this must ever be kept in mind), admit of no line or limitation, when applied to problems which pass so far beyond all human comprehension. Like in this to Time, similarly applied, their only boundary becomes that of Infinity.*

* Since the first edition of these Essays was printed, Mr. Darwin has published another very interesting volume 'On the Fertilisation of Orchids,' illustrating by close research the rarity of self-fertilisation in this curious family of plants, and the singular provisions and varieties of structure by which the conreyance of pollen is facilitated from one plant to another. As insects are almost invariably the carriers of this pollen, we have the remarkable fact of numerous and complex changes and adaptations of vegetable growth, satisfying what we may term the instinctive necessities of the plant, and at the same time those of the insect life by which this transportation is carried on. 


\section{HUMAN LONGEVITY.*}

[Edinburgh Review, Javuary, 1857.]

T/HE doctrine of M. Flourens is, that Man ought, by virtue 1 of his natural constitution, to live for a century; and that this natural term of life is abridged only by his own improvidence, follies and excesses. Such an opinion, supported by a name of some eminence, deserves consideration at least; and this we propose to give to it, adding further what occurs to ourselves as needful to a more complete and just view of the subject.

Without citing any of the innumerable maxims and current phrases by which the love of long life has been illustrated or reproved, we may at once assume the fact that all mankind, of every age, race, and country, have a deep and paramount interest in this great question of the duration of life, and of the means by which it may best be maintained and prolonged. Such maxims and speculations come to us from the earliest records of man on the earth; - they are embodied in classical poetry, in history and romance; - they appertain alike to savage and civilised life, to the fool and the philosopher; -

* 1. De la Longévité Humaine, et de la Quantité de Vie sur le Globe. Par P. Flourens, Membre de l'Académie Française, Secrétaire perpétuel de l'Académie des Sciences, \&c. \&c. Deuxième Edition. Paris, 1855.

2. On the Decline of Life in Health and Disease. By B. Van Oren, M.D. London, 1854.

3. Records of Longevity. By Thomas Bailey. London, 1856. 
and are common to every country and clime, from the arctic circle to the equator.

There needs no argument to prove that this must be so; and that the feeling is justified by being natural and inevitable. The word Life, when thus used to express the totality to every man of his present being, is itself of deep significance. While marking the longer or shorter space which each one occupies in the endless line of time, it includes those many wonderful changes of mind and body - those diverse yet continuous stages of existence - by which, without forfeiture of personal identity, childhood, manhood, and old age are all included within one span. Philosophy in every age has been occupied and perplexed by this great problem of the origin, progress, and end of life. Metaphysicians and moralists alike have lost themselves in the enquiry. Physiologists and physicians, by taking other routes, and noting the close connection of vital and physical laws, have seemed to approach nearer to its solution; yet all are met in the end by barriers which no research can surmount, and upon which speculation wastes itself in words without meaning.

Look, as a single case, to that profound problem to which we have just referred; - the preservation of unity of consciousness, under the successive natural changes of state, and the numberless accidents and strange fluctuations which compose the life of man. The phrase of Rochefoucauld, ' On est quelquefois aussi différent de soi-même que des autres,' is a feeble and superficial expression of those diversities of condition which occur in each single being, between the cradle and the grave. Yet the line of personal identity is kept entire, though thus knotted and tangled in every part of its length; - though feelings are altered and memories have passed away. If reason cannot reach these things, faith may find in them some index to a higher identity beyond the term of life on earth. 
Look again at that which seems to make the greatest breach in this unity of being, yet is itself an integral part of life, and necessary to it - the wonderful phenomenon of Sleep. If called upon to name that part of our nature, which is at once most marvellous in itself, and most prolific of conclusions beyond, we could not hesitate to find it in this great function, so familiar to our experience, so obscure to our philosophy. When Sir Thomas Brown describes sleep as 'the brother of death, which extracteth a third part of our lives,' he quaintly but strikingly denotes the wonderful fact of this periodical intermission of ordinary life; during which the senses cease in great part to have relation to the world without; and the mind, barely conscious of its own identity, works in a vague succession of images and associations; - the shadows sometimes of prior events, sometimes the seeming creation of the very act of dreaming, and traceable to no reality of waking existence. Scarcely can we abate our wonder at this phenomenon of our nature, by placing what we believe to be its true theory before us; viz., that sleep is not one, but an unceasing variety of states; - passing through every stage and degree of change from perfect wakefulness to the most complete suppression of all external consciousness ; - varying also at every moment in the degree in which each particular sense and function of life is submitted to its influence.* All these more special conditions of sleep do rather enhance the wonder and the mystery of a state, which thus occupies and engrosses a full fourth part of human existence on earth; - not less than a third, if we take infancy and old age fairly into the calculation. The sleep and various states of semi-consciousness produced by mesmeric

* This view of Sleep as a succession of ever-changing conditions, I have strongly urged in my volume on Mental Physiology, as the only one accordant with truth, or capable of expounding the phenomena, mental and bodily, of this mysterious function of life. 
or other unwonted means, we look upon with awe and amazement; forgetting, in their familiarity, those equally strange phenomena under which we periodically pass this portion of our existence.

We have hitherto been speaking of Life in its larger physiological sense. We now come to that more especial view of it indicated by the title of the volume before us; in pursuing which subject however, it will still be needful to recur occasionally to the more general theory for aid and illustration. The questions regarding human longevity are far too complex to be submitted to any single solution, or separated from the elementary consideration of life itself.

The name and repute of M. Flourens in the scientific world might well warrant some high expectations of a work coming from him, and bearing this title. We are bound to say that these expectations are in no way fulfilled by the treatise before us; the greatest merit of which is that it is not long, and not otherwise tedious than through its loose and inconsecutive reasoning. The result he seeks to establish as to human longevity we consider to be unfounded; and his arguments on behalf of it vague and unsatisfactory. Of this first part of his work we shall speak more in detail hereafter. Meanwhile we may content ourselves with a very cursory notice of the latter half of the volume, indicated in the titlepage by the phrase 'De la Quantité de Vie sur le Globe.'

Though aware that this phrase is borrowed from Buffon, we still claim the right to object to it here, as an affectation of higher philosophy and originality than really belongs to this portion of M. Flourens's work. The doctrine of Buffon, upon which his own views are founded, is this; - that taking all created beings into account, the total quantity of life on our earth is always the same; - that the Creator has brought into being an incalculable number of living organic molecules, indestructible and common to all forms of organic life, the 
material of generation, growth, and duration of existence;that death, while it destroys and dissolves individual forms or lives, does not annihilate these molecules, which pass into other forms, giving and maintaining life as before, and being always the same in total quantity on the globe. This is one of the many fruitless hypotheses common to every age; easy to construct - difficult absolutely to refute or deny -impossible to prove. The molécules organiques vivantes of Buffon, like the plastic nature of Cudworth, are an effort to shelter penury of knowledge under the garb of scientific language. Though the higher genius of Leibnitz gave somewhat more of philosophy to his monads, yet essentially the attempt and the failure are the same. Life may be defined, more or less justly, through its functions. In its elements, no reason or hypothesis can reach it.

M. Flourens, however, while professing admiration of Buffon's genius, and acquiescence in the hypothesis as to the equal and constant quantity of life on the globe, places this doctrine on a basis of his own, which we may briefly give in his own words :-

Je n'étudie la vie, ni dans les molécules organiques, ni dans les monades. J'étudie la vie dans les êtres vivants; et je trouve deux choșes: la première que le nombre des espèces va toujours en diminuant, depuis qu'il y a des animaux sur le globe; et la seconde que le nombre des individus, dans certaines espèces, va toujours au contraire en croissant; de sorte que, à tout prendre, et tout bien compté, le total de la quantité de vie, j'entends le total de la quantité des êtres vivants, reste toujours en effet à peu près le même.

No evidence for this doctrine is known to us, and assuredly none is furnished by our author to justify its enunciation as a special discovery. It is very true, as M. Flourens states, that various species of animals - some of them, as attested by their bones, of gigantic size - have become extinct during what may be called the existing epoch in the history of the 
globe; - that others are probably in progress towards extinction; - and that no new creation is known within this period, of animals of equivalent size to replace those thus vanishing from the earth. It is also true that many of the domesticated species, serving to the uses of man, have been largely increased in numbers in effect of the increase of human population on the globe. But these things, which are true, are not new; and the doctrine derived from them - that a balance is struck between the two opposed conditions, and that the total quantity of life, or of living beings, remains always nearly the same - is one wholly unsupported by the premises. Its wording, in fact, betrays the author's hesitation as to its truth. The ' $\grave{a}$ peu près' is a great discounter of realities in science, as in most other things.

It will be obvious indeed to all who care to reason on the subject, that we have no knowledge, or means of obtaining it otherwise than by vague approximation, as to the total quantity of life on the globe, or the relative quantity at different periods. Such phraseology then, except as denoting mere hypothetical questions, cannot rightly be admitted into scientific language; seeing especially how little we are able to estimate numbers or individualities of life in any of the great classes of the animal kingdom; - how impossible it is to conjecture them in the multitude of those lower forms which we reach only through the eye of the microscope. Nor in fact can any such conclusions as those put forward by M. Flourens be accepted, as long as doubts exist as to the proper definition of species, and the possibility of their change or transmutation in long periods of time. We may not acquiesce in these doubts, but the question is one fully open to future enquiry.

Dismissing however this subject, which it is not necessary to pursue further, we come to the main topic of M. Flourens's 
volume - the longevity of man. We wish to deal fairly with his doctrine, and shall state it as clearly as we can. But here again we have to complain of the loose and desultory character of his reasoning, broken by numerous citations from other writers, poets as well as physiologists, and many of them little fitted to serve as authorities in a scientific treatise. We have been accustomed to look into the pages of Molière, Voltaire, and La Fontaine for satire upon human life, and not for sober reasoning upon longevity.

M. Flourens propounds his main question in these terms: - 'Quelle est la durée, naturelle, ordinaire, normale, de la vie de l'homme?' And he instantly replies to this question by a passage from Buffon, which he takes as the text and authority for his own views. 'L'homme qui ne meurt pas de maladies accidentelles, vit partout quatre-vingt-dix ou cent ans.' - Though we might comment on the tautology of naturelle and normale as applied to the term of life, we can find no other fault in this manner of propounding the theme. In adopting the conclusion of Buffon, he follows the same train of reasoning to it. He affirms that the duration of life depends neither on climate, nor food, nor race, nor any external condition; but has relation solely to the natural constitution and intrinsic vigour (vertu intrinsèque) of the vital organs. Regarding everything in the animal economy as submitted to fixed laws; - that every animal species has its determinate shape and size, its particular time of gestation and period of growth; - he infers that the natural duration of life must be equally definite for each species, and open to determination. Still following Buffon at each step, M. Flourens accepts his doctrine that this measure of life is to be found in a certain proportion to that of complete growth, as well in man as in other animals. He differs only as to what may be taken as the term or limit of this growth. Buffon, naturally enough, makes stature his index; 
and assuming the average period of growth in height in Man to be about sixteen years, he takes six or seven periods, the multiples of this time, to express the natural duration of human life. He fortifies himself in this result, by noting a certain proportion of time of growth to the length of life in other animals, as the horse, dog, stag, \&c.; but neither numerically nor otherwise does he claim for his doctrine the absolute exactness of a physical law: "The whole duration of life may in some measure be calculated by that of the period of growth. Man, who is fifteen years in growth, may live six or seven times that period of time.'

M. Flourens is bolder in his conclusions, and in the same degree farther removed from truth. He adopts as the term or limit of bodily growth (accroissement) the complete union of bones at their Epiphyses (an expression we shall speedily explain), and alleging this consummation of growth to occur in man at the age of twenty; and in certain other animals at other ages, but in each respectively the fifth part of the term of life, he at once multiplies by 5 the 20 years of human growth, and pronounces 100 years to be the natural period of human existence. We produce this view in his own words :-

Buffon says that every animal lives about six or seven times as long as it is in growing. On this supposition the relation would be as 1 to 6 or 7 ; but the real relation of the period of growth to the duration of life is as 1 to 5 , or nearly so. Man is 20 years growing, and he lives five times 20 years, or to 100 . The camel is 8 years growing, and he lives to 40 : the horse 5 years growing, and he lives to 25 ; and so on to other animals. We have thus then, at last, an accurate criterion which gives us with certainty the period of growth. The duration of that period gives us the duration of life.

The argument, thus put, is more summary in manner than satisfactory in substance. We doubt much whether this period of epiphysis, or completion of bony union, has been 
determined in a sufficient number of animals, and with sufficient exactness, to serve as a basis for numerical results. We believe further that the relation of this period to the normal duration of life in different animals, is nothing more than that general proportion which every successive period bears to its antecedents and consequents; rendering each in some sort a measure and index to the rest. What is called epiphysis is a very limited phenomenon of growth; and though seemingly the last in the series of osseous developements, cannot be admitted as an epoch in life, or as having any important relation to other structural changes. We dispute then, altogether, the right of M. Flourens to take it as his basis; and by applying his multiple of five, to make it tally with what is evidently a foregone conclusion of his own as to the length of life. This conclusion is not logically attained, and is manifestly contradicted by facts.

He appeals, however, to actual experience on behalf of his doctrine that one hundred years is the natural life of man; and that its curtailment below this normal term is the result of those errors and excesses in the manner of living, which impair the organs and produce premature decay. And his argument here mainly lies in the citation of those cases in which life has been prolonged far beyond the average limit; - instances often of exaggerated or doubtful kind, but yet numerous and authentic enough to be admitted as positive facts in the natural history of man. While justly sceptical as to examples which go beyond our own experience, we cannot dispute the statements coming to us from various sources, from different countries and periods of time, that human beings have occasionally reached, and now and then exceeded, the extraordinary age of 150 years. In our own country, for example, though we may put aside as unproved the case of Henry Jenkins, alleged (chiefly on his memory of the battle of Flodden Field) to have lived 169 years; and 
regard with doubt that of the Countess of Desmond, whose age is recounted at 148; yet we cannot equally reject the evidence as to the 152 years of Thomas Parr's life, accredited as it is by the testimony of Harvey, who examined his body after death, and states that there were no obvious reasons why he might not have lived yet longer, but for those changes in his habits of life which followed his removal to London and to the kitchens of the palace.

Instances of this extraordinary kind, indeed, are fully admitted by some of the most eminent physiologists; and Haller and Hufeland respectively, after citing several especial cases of life exceeding 150 years, affirm it as probable that the organisation and vital forces of man may be capable in some cases of reaching 200 years of age. No proof, however, is given by them of such age having ever been attained; nor is there any record of it, except in one or two instances, so utterly without attestation that they must at once be discarded. We note one of them below, simply to show how loose is the evidence often received on these matters. ${ }^{*}$ But we cannot refuse belief to a certain proportion of cases in those Registers of longevity which, comprising many thousand instances, must contain some average of truth, however difficult it may be to discriminate or define it. Dr. Van Oven, in tables which he has drawn up with great diligence, gives seventeen examples of age exceeding 150 years. In another of the volumes before us (the 'Records of Longevity,' by Mr. Bailey) we have a catalogue of three or four thousand cases of longevity verging closely upon, or exceeding 100 years, and several of them reaching 150 years. Without seeking to impeach the good faith with which these tables are constructed, we cannot but feel the great want of any

* This case is derived from a parish register in Somersetshire, where the record appears of the 'burial, in Dec. 20, 1588, of Jane Britten, a maiden as she affirmeth at the age of 200 .' 
exact or sufficient evidence belonging to them; a fault which no present research can now repair. Still we are bound to admit some, even of the extreme cases, as authentic; and to presume an increasing proportion of others, which, though lower in the scale, do yet greatly transcend the average term of life. We shall have occasion afterwards to refer to these more especially. At present it is enough to state that we have sufficient proof of the occasional prolongation of life to periods of from 110, to 130, or 140 years; - cases which, thus far authenticated, we must necessarily take into view when dealing with this question of human longevity. But in so doing, we are called upon to submit them all to the great general law of averages, and not to propound them, as M. Flourens does, as exponents of the natural capacity for life in man. We might just as reasonably assert that six feet is the natural stature, because some men have reached the stature of eight, or even nine feet; - or on the other hand, that four feet is the normal measure, because Count Benyowsky and the American Tom Thumb were dwarfed down to two and a half or three feet; - or yet further affirm that fifteen stone is the natural weight of the species, because Daniel Lambert, and some of his brethren in obesity, have nearly doubled this weight. The real fact is, that these anomalies, either of excess or deficiency, occur in every part of the physical structure of man, as in every part of the world of nature that surrounds us. Exceptional however to the general laws which govern the animal creation, they are continually checked and controlled by these laws. They cannot pass certain limits without bringing into action fresh physical causes, tending to destroy the anomaly, and to restore that particular condition, which, as far as we can see, is specifically annexed to every organism in the natural world.

Here then we find the value and import of the great law of averages, to which we have just alluded. Almost it may be 
called a new method of research, though now among the foremost in contributing to the extension and exactness of human knowledge. It is curious, but true, that the understanding of this particular mode of reaching truth was never fairly attained by the philosophers of antiquity. Some practical application of it is, indeed, next to inevitable in the most common processes of human thought and enquiry. But the principle was never fully recognised or converted to scientific use ; - an intellectual anomaly having close kindred with another still more singular default in the ancient philosophy; viz., the want of any right appreciation of experiment, as the most certain and powerful instrument of scientific research.

Modern science has fully acknowledged, and carried into active use, these two great methods; mutually dependent in every part and principle of their application. The law of averages, indeed, has acquired of late a wonderful extension and generality of use ; attaining results, from the progressive multiplication of facts, which are ever more nearly approaching to the fixedness and certainty of mathematical formulæ. Every single observation, and every new fact added, comes into contribution to these resulting truths. Phenomena, seemingly the most insulated, and anomalies the most inexplicable, are thus submitted to laws which control and govern the whole.

Many of our readers must be aware how largely this doctrine of averages has been applied, not merely to physical phenomena, but even to the social and moral conditions of man, as the highest tenant of that globe on which his destiny is cast. Under the auspices of Quetelet and others of his school, aided by the facilities of intercommunication now existing between the different regions and races of the earth, statistical knowledge thus attained has taken a high place among the sciences, and promises for the future a powerful influence on the welfare of mankind. 
Previously, however, to this more scientific direction of the doctrine of averages, it had already received what we may call a mercantile application, in relation to the very topic now before us; viz. the estimate of human longevity. A new and vigorous traffic has sprung up within the last eighty years, of which the duration of life is the subject and basis. We need not enlarge here upon the principle or history of Life Assurance as a branch of actual business. Originating in England, it is here especially that it has grown and diffused itself so as to become an essential part of our social policy; a natural, if not necessary, result of those complex relations of property and family connections, which are created by high civilisation, personal freedom, and political security. And though partially defaced by certain evils inseparable from all human institutions, we may regard the system as one conducing largely to the interests and welfare of the community. As interpreters of the averages of life, and of the various conditions affecting its duration, Insurance offices in their present extension have become our best guides; and their tables and calculations, though modified materially of late years, do singularly expound that uniformity of results which arises out of these numerous and extensive records of age and death. Mathematics have lent their aid to the calculation, and given to it many of the conditions of an exact science.

Still more important documents as to human longevity are those furnished by the English Decennial Census, and the annual reports of the Registrar-General. In these the results are derived from the total population of the kingdom; and so admirably recorded and classified as to afford not solely numerical conclusions respecting the several periods of age attained, but also much and curious knowledge as to the circumstances and conditions which affect the average duration of life in different classes. Those who desire more detailed information on this subject cannot do better than 
consult these valuable reports. They possess the further advantage of being readily brought into comparison with the corresponding tables, more or less perfect, furnished from other European kingdoms and from the United States; many of which tables are in fact contained in the volumes of English registration.

We shall have occasion to allude to some of these reports hereafter. But meanwhile we may state, as the result from them, that they utterly refute the doctrine which forms the pith and purport of M. Flourens's volume. Such formal refutation was in truth hardly needed of an opinion contradicted, as we have before said, by the common experience of mankind of every age and country. A hundred years is not, and has never been, the natural or normal age of man. No deviations from a life of nature, - no excesses of luxury, or labours and privations of poverty, - will suffice to explain the disparity between the doctrine in question, and the facts as they stand in face of it. M. Flourens dwells with complacent detail on the old and familiar instance of Louis Cornaro, the Venetian self-reformer; - a notable one, doubtless, if we may trust to its correctness in all particulars; but bearing marks of exaggeration; and at best but an individual case, where the argument needs a multitude. If seeking for any causes likely to affect and alter the term of life on a large scale, we should rather expect to find them in the extraordinary diversity of physical conditions to which mankind are exposed; and especially in those conditions which belong: to the extremes of climates in different parts of the globe. But M. Flourens himself rejects these causes as of little or no influence upon what he assumes as the normal term of life; and though we dispute his doctrine on the latter point, we agree with him so far as to believe that the external physical conditions to which man is subjected, have less influence than might be supposed upon the average duration of existence. 
Adaptations, partly of bodily textures, partly of alimentin each case the effect of these physical conditions - come into action here, and restore that parity which appears to be the natural law for the species.

But it may be asked, - What then, rejecting this doctrine, is the true natural term of human life? Or is there any which can be strictly designated as such? The question is a simple and definite one. The answer cannot easily be rendered so. It might seem probable indeed primâ facie that in the case of Man - single in species and presumably derived from a single stock - there should exist some middle term of natural age, marking the destined duration of his being on earth, apart from all those incidents, physical or moral, which crowd upon and affect his existence. But these incidents are so numerous and varied, so obscure often in their origin and progress, yet so constantly blending themselves; as we shall see hereafter, with the hereditary constitution of families and communities, that all sagacity is at fault in seeking to deduce and strictly to define such natural term of life. The most copious and accurate registers fail us here; and we are forced to regard it in some sort as an abstract conception incapable of being expressed, otherwise than approximately, by any simple number. If called upon to state this approximation, we might perhaps seek to rest it on the venerable record of 'three-score years and ten;' with a leaning, however, to the belief that this is rather below the true mark. Blumenbach, a great authority on such subjects, rated the period at eighty years. But under no circumstances can we admit the Century of years which M. Flourens has pleased himself by assigning to life; or allow the force of the arguments by which he seeks to substantiate his doctrine.

Dismissing then this particular question suggested by the work before us, we may invite our readers to other parts of the interesting subject of human longevity. And first there 
comes into consideration the curious topic of comparative longevity; - comprising questions of nations and races, civilised and savage; - of successive periods in the history of the world; - of families as well as individuals; - of sex, occupation, climate, food, and all other conditions pertaining to human life. Volumes would be needed to follow these matters into detail. M. Flourens touches upon them so slightly and vaguely, that no aid is to be gathered from his treatise. In our limited space we cannot bring them before our readers otherwise than by selecting a few prominent conclusions, serving to illustrate the main objects of enquiry, and the methods best fitted for attaining them. Any attempt to go beyond this would be perplexing and futile.

It is natural to look first - and it cannot be done without interest-at the records of longevity in former ages which may be brought into comparison with those of our own time. We have already indicated as the chief attainable results of such enquiry, the general duration of what may be considered the completed life of man, and the extreme cases of age stretching beyond this mean term. For obvious reasons we omit all reference to the length of antediluvian life, as we receive it from the Scriptures. We could say nothing new towards the solution of this question; depending, as it does, upon conditions and a state of the world to which no present knowledge can apply. We turn with more assurance to another record, (of later time, but also bearing in its superscription the great name of Moses), which, in defining the life of man at three-score years and ten, affords a measure corresponding closely, as we have already seen, with the every-day experience of our own age.* In the beautiful

* It is worthy of remark, though doubtless familiar to many, that from the time of Noah to the days of Moses and Joshua, the record is one of successive and gradual decrease in longevity. Joshua 'waxed old and stricken in years' some time before his death at 110 years. 
passage of this psalm (so admirably translated by Lord Bacon), we have in a few words the touching picture, true to every time, of the decrepitude and other ills which affect life when prolonged beyond the average term the Creator has assigned to it.

The records of ancient Egypt, Assyria, and Persia, though not expressing the fact to us in such positive forms, yet concur in furnishing the same general inference. The several periods of individual life are denoted as we now denote them; and generations succeed one another, as far as we can interpret the ancient monuments of the dead, under an equal and similar law. The pyramids were the tombs of monarchs who, as an old writer says, 'astonished Heaven by their audacities,' but whose term of existence would seem to have been strictly commensurate with our own.

As we descend to the days of Greece and Rome, the notices derived from history and other kindred sources become more explicit and particular. The result we may affirm to be the same; testified to us both in the mean term of life as we have defined it, and not less remarkably in those deviations by excess, which in themselves furnish a sort of reflex proof as to the average; - a manner of verifying the mean number more valuable than on first sight might appear. Though we still cannot authenticate particular facts in these periods with the same assurance as by the statistical tables of our own time, yet neither Greeks nor Romans were wanting in methods of assigning exact date, even to the events of private life, through the popular and political institutions which are so deeply embedded in their history. The Olympiads and public festivals of Greece, and the Consular Fasti of Rome gave time, as well as name, to numerous family and personal occurrences. 'The 'calidus juventâ consule Planco' of Horace, is a familiar instance of the mode of dating events through this greatest and most lasting institution of the 
Roman State; and we know it to have been applied to that record of births which is essential to all evidence regarding longevity. We may refer, as an example of the completeness of some of these documents, to the account Pliny gives us of the Italian census instituted in the time of Vespasian; in which, to some extent at least, the classification by ages seems to have been conducted on the same plan as that we now follow. To this author we are indebted for much information on the subject of longevity; and the caution he applies to such instances as are presumably fabulous, or dependent on other modes of estimating years, is some security for the exactness of the cases which he admits without such suggestion. They are probably as authentic as the similar records of any modern people two or three centuries ago.

Premising this, we may mention a few of the particular instances of longevity belonging to those times; and such especially as are associated with the eminent names of antiquity. The first case, indeed, that of Epimenides the Cretan (the subject of the famous example of the circulating syllogism), we must regard as very doubtful; the authorities for his alleged age of 167 being exceedingly slender. The Greek philosophers generally, however, of all sects and tenets, make fair show on the list; and under better evidence of authenticity. Zeno is stated to have lived 102 years; Democritus, 104; Pyrrho, 90 ; Diogenes, 90 ; Hippocrates, 99 ; Plato, 82 ; Isocrates, 98 ; Gorgias, the master of Isocrates, 107. But for the cup of hemlock, and the sword of the Roman soldier, the 70 years of Socrates and the 75 years of Archimedes might well have reached the same high class of longevities. The old age of Sophocles, 90 years, is associated with the touching anecdote of his recitation of verses from the CEdipus in Colonus, in proof of his sanity of mind at that age. The lofty lyric genius of Pindar was not lost to 
his country until he had reached 84 years. Simonides wore his elegiac laurels to the age of 90 .

We wish we could settle a much disputed question, by assigning a period and time of life to that greatest of all the Greek poets, whose name alone lives, but will ever live through every age of the world. Strange it is that the fame of Homer, thus immortal, should be dissociated from any distinct record or time of birth. Still stranger, perhaps, that his individuality should have been called into question by the hard and technical criticism of our own day.

We might go on to cite numerous instances of eminent longevity from the Roman annals; many of these on the authority of Cicero himself. But we will confine ourselves to a few cases of female longevity, of less historical weight, though curious in other ways. Terentia, the wife of Cicero, lived to 103 ; Clodia, the wife of Ofilius, to 115 years. Two remarkable actresses stand on the list; one of whom, Lucceia, is stated to have performed as Mima for about 100 years; the other, Galeria, was brought back to the stage, during games celebrated in honour of Augustus, in her 104th year, and 91 years after her first appearance before the Roman public. Pliny affords us a similar instance from the other sex, that of the dancer Stephanio (qui primus togatas saltare instituit), who having danced at the secular games of Augustus, performed again at those of Claudius, 63 years later, and lived still some time afterwards.

The Census, already mentioned as instituted by Vespasian, furnishes some results as to longevity singular enough to make us more than doubtful of their truth. The instances given by Pliny are taken exclusively from the region between the Apennines and the $\mathrm{Po}$; and upon the record of this census (which he himself calls res confessa) he enumerates 54 persons who had reached the age of $100 ; 14$ of 110 years; 2 of $125 ; 4$ of $130 ; 4$ of 135 ; and 3 of 140 years. 
In the single town of Valciatium, near Placentia, he mentions 6 persons of $110 ; 4$ of $120 ; 1$ of 150 years. These round numbers convey suspicion as to the reality of the ages in question; and the whole statement, drawn from a district by no means noted for its salubrity, is so much in excess of any similar record in other countries, that we must repeat our expression of entire disbelief. If true, it could only be solved by supposing a remarkable aggregation of cases of hereditary family longevity, through intermarriages in the same province. Nor can we draw from these, or other memorials of ancient longevity, any argument for believing that the mean duration of human life was ever above that which belongs to the period in which we now live.

From the question of comparative longevity in ancient and modern times, we come to that of races of men; - an enquiry subordinate in some sort to the higher question, whether we must regard all races as derived from a single primitive stock? or whether, to explain the remarkable physical diversities which we see, it is needful to suppose the original creation of more than one type on the earth? This question is one which has been keenly agitated of late years. For ourselves, we assent to the former belief, or that of unity of type and origin. We find evidence for this in the very multiplicity of existing varieties; and in the manner in which they graduate into one another. Of abrupt lines of demarcation there are none; and we follow the Negro into the Caucasian races through every step and stage of variation. This argument may not be positive; but it at least answers the objection from the disparity of the extreme cases.

We must not, however, be seduced from our subject by this higher enquiry, curious and important though it be. It is enough to recognise here that Man forms, in every proper sense in which the term can be defined, a single and separate species in the animal creation. The varieties or 
races of this species are all related by common conditions of reproduction, - of structure and functions of the skeleton and internal organs, - and of the aliment appropriate to growth and maintenance. To these varieties thus far identified in character and origin, our present question of comparative longevity applies. Without referring to those several divisions into races, which historians or ethnologists have adopted, we may at once take the extreme instances of the Negro and of the people of Northern and Central Europe, as including all intermediate cases. And here again, as in the question regarding the comparative longevity at different periods of time, we are led to the conclusion of that general parity, which the conditions just noted might lead us to expect. We cannot, indeed, go for facts to parish registers of Bornou, or to Statistical Societies of Soudan; but from the registries of our West Indian Islands, and from the decennial census of the United States, we obtain information bearing closely on the question before us. We must not say deciding it; since the results, if indeed certain, would show a very singular superiority in length of Negro life over that of European origin. In 1840 , for instance, when the population of the United States was about 17 millions, of which $2 \frac{1}{2}$ millions in round numbers were negroes, the Census gave 791 as the number of whites above 100; while of slaves the number of those above 100 is registered as 1,333; of free negroes as 647 . In 1855, we find from the Census, that 43 persons died in the United States above 100; the oldest white male at 110, the oldest white female 109; the oldest negro man 130, the oldest negro woman 120, both slaves.* From

* While correcting this sheet for the press, we see in an American paper the statement of the death, at Rummerville, in Virginia, of Mr. Craft, a servant of Washington in the war. of 1756, at the age of 128 years; leaving two sons living, the youngest 97 years old. Other instances of great longevity are noted in the same family. 
Professor Tucker's analysis of the American census from 1790 to 1840 , published a year ago, we derive the strange result, if true, that the chances of living above 100 are 13 times as great among the slaves, and 40 times as great in the free negroes, as in the white population of the country.

These results however, as we have just hinted, are too anomalous to be readily accepted. Scarcely half a century has elapsed since the importation of slaves from Africa was prohibited by law; and we may therefore safely presume, that most of those whose alleged ages exceed 100, were of African birth; a circumstance which bars in limine all certain conclusions on the subject. Even with respect to those born in the States, there is much likelihood of faulty registration, added to by the frequent transference of slaves from one estate to another. And yet, further, we have to consider here the habits of the negroes themselves; their curious inaccuracy as to all matters of numbers; and their proneness to exaggeration, especially when by applying this to age, they may hope to obtain some interest in their fate, or mitigation of their labours. Professor Tucker goes farther, and speaks of the temperate and easy life of a large part of the slave population as adding to the chances of longevity. We should gladly believe that it was so ; but the much larger proportion of centenarians among the free blacks weakens the force of the inference.

For these and other reasons we cannot draw just conclusions from the American census; while the general evidence from other sources (confirmed by personal enquiry we have ourselves made in the country) leads to the belief that the average longevity of the Negro and European races differs but little in amount. The extreme cases of longevity in the former, furnished us from our own West Indian Islands, closely tally with those recorded in the registers of the white 
races of Europe; and, as we have already said, these extreme cases form a sort of index to the average sought for.*

We have not space to pursue this comparison in detail through the several races and nations of modern Europe and Asia, though the materials we now possess are ample for the purpose. Of these races, variously commingled indeed in their present nationalities, the Teutonic and the Sclavonic are the most considerable; derived, as modern philology teaches us, from a common Asiatic source, yet with wide separation by intervening time. European Russia best expounds all that relates to the Sclavonic race. In the subjoined note we give a few particulars, drawn from the registers of this empire, and also from those of some of the Teutonic nations of the north of Europe $†$ They confirm the result of general equality, both as to the medium duration of life, and as to longevity by excess. The medium annual mortality varies materially indeed in different countries, still more in detached localities; but such diversities

* We have attempted, but without success, to obtain some distinct eridence as to the comparative longevity of mulattos, quadroons, \&c. The common belief is that they are short lived, and that such breeds soon cease to be prolific. But more and better attested details are required before we can reach any certain conclusions.

+ For Russia, the returns of 1842 are before us. From these it appears that the mean annual mortality in that empire is fully $3 \frac{1}{2}$ per cent. (in the provinces which include the basins of the Wolga, Don, and Dnieper, considerably more), a very high ratio compared with the $2 \frac{1}{4}$ per cent. of England; but in some part explained by the great mortality of infants in Russia. These tables do not give detailed specification of ages above 90 ; but they record for several years the number of deaths of males upwards of 90 , giving a mean of more than 5,000 for each year, or probably 10,000, had females been included. This stands in large proportion to the population; but as at the date of 90 years before these returns, there was no system of registration in Russia, their accuracy admits of much doubt. In Austria, including Lombardy, in 1842, 446 persons died at ages above 100 , out of about 460,000 deaths.

In the Prussian States, in 1841, 786 males and 890 females died at ages upwards of 90 . In Norway, in 1845, when the population approached 1,200,000 there were found to be 19 males and 22 females above 100 . We could have wished for some specification of the actual ages here, Norway being reputed to afford examples of extreme longevity. 
often belong to particular periods of life only (as that of infancy), and affect more partially than might be supposed the result with which we are here mainly concerned.

We have already spoken of the excellence of our recent English registration, as attested by the volumes annually published. Though they afford us no present cases of longevity equivalent to those of Jenkins and Parr, they indicate a medium duration of life, and a proportion of lives above 100, at least equal to what exists in any other country. Taking two recent years as a brief illustration, we find in 1852, when the population of England and Wales was 18 millions, there died 35 males above 100 years of age, the oldest 105 ; - and 53 females, of whom three reached 106, one 107, and two 108 years. In the following year, the register tells us of the deaths of 31 males, and 62 females, above 100 ; - the oldest male 109 , the oldest female 110 years.* We may add regarding England, that the low rate of medium annual mortality (not exceeding $2 \frac{1}{4}$ per cent., and much below that of most European nations) expresses causes which must undoubtedly have effect in multiplying the cases of great age. As we have already said, the influence of these causes is limited by various considerations; but we cannot reasonably exclude it; or deny that there may exist from this source certain mean differences of longevity in the several races and nations of mankind, as with respect to stature and other marked features of bodily conformation. The evidence is not yet sufficient to define these differences, or the precise conditions producing them. The reasons, however, already urged lead us to believe that the disparity, when ascertained, will not be found considerable in amount.

In pursuing this question of human longevity, we pass

* The returns recently issued for 1860 , show that in that year 22 men and 47 women died, who had reached, or gone beyond, 100 years of age. One woman, in Glamorganshire, reached 111 years. Two men are recorded as having each lived to 107 years. 
from races and nations, where large averages cancel more or less completely all subordinate inequalities, to the lesser divisions of families and local or limited communities, where new causes come into action, no longer neutralising each other in their general results. All who rightly comprehend the law of averages will see at once that this must be so. It is impossible to particularise the many causes which affect the health and life of man in various localities; but the subject of longevity in families connects itself with one of the most curious questions in human physiology - that of the hereditary transmission of physical qualities and peculiarities from parents to offspring. Every one is familiar with this fact in the case of other animals; especially in those domesticated by man, and made more useful to him by the varieties thus produced. We cannot affirm that the capacity for change by such hereditary transmission, is as great in man himself; for except in the instance of the gigantic Prussian grenadiers, and possibly in the usages of some savage tribes, we are not aware of any attempt distinctly made to test this question. But in one form or other the fact is familiar to the observation of all; subject, indeed, to the anomalies which beset every part of this great mystery; yet exhibited in such numberless ways, on mind as well as body, as to show its mighty influence on the destinies of man. The most minute peculiarities of external feature, as well as the grosser conditions of stature and bulk, are capable of being thus transmitted; and we cannot doubt, upon pathological observation, that the internal organs also possibly even that wonderful fluid which circulates through and ministers to all of them - are subject to the same influence derived from one generation to another. The bearing of this influence on the formation of national diversities of feature and character, is a most curious collateral topic, but its discussion would be out of place here. 
What, however, clearly pertains to our subject, is the fact of longevity being hereditary, and running in particular families and lines of descent. Where the organs, serving to the great animal functions - respiration, circulation, nutrition, and secretion - are sound, and conveyed as such from one generation to another, an average prolongation of life will occur as the natural effect. All this is fully confirmed to us by common observation. Every group of tombstones indeed tells a true tale, in this respect, of what lies underneath. Before the Titanic power of steam had given the speed and vehemence of the race-horse to our manner of travelling, we can remember the time when we used to loiter through the country churchyard while horses were changed, or dinner prepared, at the road-side inn. Here, in its simplest but most touching form, may well be learnt the truth of which we are speaking. On one family group of gravestones are recorded the many early deaths which give evidence of feeble or diseased family constitution; - while another group, close at hand, tells in its dates the history of sound native temperament and prolonged age from parents to offspring. Considering the various collateral influences ever at work, we have often been surprised by the uniformity of this result. But these external influences are in fact continually tending to restore the balance; and do in the end retrieve that average in which all anomalies and inequalities finally merge. Intermarriage among different families, in successive generations, is obviously the natural provision against such inequalities. They sometimes, however, continue long; and occasionally assume very singular and morbid aspects; where, from some cause of local or social limitation, intermarriages are confined to a small community without due admixture from without. This natural correction then, very little aided by human prevision, is a providential one;-illustrated by analogies in other parts both of the animal and vegetable 
kingdom; but a mystery in itself, like all that belong's to the transmission and interblending of life through successive generations.

Descending from families to individuals - the last step in the scale we have thus rapidly been following - we find the contingencies which affect longevity to multiply largely and become more obvious, than when hidden under the averages of larger numbers. One important classification, however, here suggests itself, viz. that of the Sexes. Though it would be difficult to prove the point absolutely, we think it may be presumed that the natural term of life is the same in man and woman. It must be admitted, indeed, that our own Census, in common with that of many other civilised communities, shows a considerably larger proportion of females than of males attaining 100 years. But we believe the fact to be of ready explanation, without recurring to any recondite causes. Woman drops more easily than man into the passive existence of advanced age. The pursuits, pleasures, and passions of her antecedent life are for the most part of a more tranquil kind, and do not so strongly contrast with the inert seclusion of later years. The expression of a French writer 'Peu de gens savent être vieux,' has much closer application to one sex than the other. The incidents of childbearing might seem to lie on the other side. But these belong to, and affect, an earlier period of life; and can hardly be considered as equal in influence to the external casualties which more especially beset man, even to the extremity of age. We may further remark that the cases of extraordinary longevity, which we have denoted as a sort of index to the average, are found to give a general equality of result for the two sexes.

We come then finally to individual life in reference to longevity; - a subject which cannot be dealt with except under new conditions, and a larger reference to physical and. 
moral causes in their influence on the animal economy of man. Of hereditary temperament we have already spoken. But apart from this, the whole of life teems with personal incidents which must needs affect, more or less, its duration. Every particular variation of health, however produced, has some definite relation, perceptible.or not, to this result. The physical conditions and habits of the individual, whether those of luxurious sensuality or of meagre poverty, are in constant action here; and associated with these the various occupations, whether of choice or of necessity, which minister to the livelihood of man. No argument is needful to show the bearing of the latter both upon individuals and communities. In a manufacturing and commercial country especially, where population is crowded, and where art and labour in their every branch are strained to the utmost reach of human exertion, life becomes subject to influences which act powerfully upon it, and tend on the whole to shorten its duration. The materials and documents we possess are not yet copious and exact enough to justify more certain conclusions on the subject. That some particular occupations abridge life, by bodily confinement, privation of good air, the direct action of noxious vapours and other causes, is a fact too familiar to all. To this class of causes, acting thus definitely, must be referred in part the difference between town and country longevity; testified in England by the mean annual mortality in the larger towns being twenty-six or twenty-seven in a thousand, while that of the whole kingdom does not exceed seventeen in the same number. The whole subject is one of high interest to our social welfare, and attention is now keenly awakened to it.

Curiosity may also be directed to the question how the learned professions stand as to relative longevity? In such an enquiry it is obvious that individual cases go very little way towards its solution. With respect to the profession of 
Law, we have no connected evidence sufficient to warrant a general conclusion, though many particular instances at once suggest themselves of great judges, who have continued to render eminent public services through a long term of age. The Insurance Offices, until recently at least, gave more distinct results as to the value of clerical life in England. The Clergy, in truth, have formed their highest description of insurances; affording an average of life considerably beyond that of any other class. We have great reason, however, to doubt whether the hard-worked clergyman of the present day will maintain this average for the future. The Medical profession, both in England and elsewhere, comes much lower in the scale of longevity. No material for satire can be drawn from this fact. The hard labours, broken rest, and anxious responsibilities of medical men, and their much greater exposure to infection and other causes of disease, well explain that, while seeking to prolong the lives of others, they are often shortening their own.

The longevity of statesmen, and of men of letters, forms another curious topic of enquiry; but of greater difficulty from the more doubtful definition of these classes. Here again we must reject the evidence of particular cases, as not leading to any certain conclusion. We read of Henry Dandolo reaching the age of 97 ; Cardinal Fleury, 90; Bolingbroke, 79 ; Alberoni, 80; Pombal, 83 years. In our own times we are familiar with the renerable aspect and antique manners of Talleyrand, Metternich, and Nesselrode - statesmen who have played so various a part amid the changes of dynasties and the conflicts of empires. And again, among the greatest men of our own country, less exposed indeed to revolutionary storms, we find the names of many who, happily for this nation, have continued the eminent labours and services of earlier life into a prolonged age of honour and usefulness. Of the Duke of Wellington 
it was well said on the morrow of his death, that he had exhausted nature as he had exhausted glory. And to the same generation belong the names of Lansdowne, Lyndhurst, and Brougham,- still maintaining, under weight of years, the native vigour of mind, eloquence, and ability which have marked every stage of their career. But, after all, such cases interpret only individualities of temperament, and afford no answer to the general question. The same is true in the case of literary and scientific men. We might quote the instances of Hobbes, Voltaire, Fontenelle, Heyne, Goethe, Newton, Kepler, Halley, Cassini, Maria-Agnesi, Humboldt, and others, all reaching ages between 80 and 100; but we can venture no general affirmation grounded upon a few examples of this kind.

From these topics we pass to another of close affinity with them, viz. the influence of those passions, cares, and excesses of mind which belong to the strange and fitful history of human life; and affect every part of it, from very infancy to the extremity of age. It would not be easy to estimate the relative influence of moral and purely physical causes upon longevity. They are so mutually consequent upon one another, so interwoven in all ways, that reason is perpetually at fault in seeking to distinguish their effects. What we have hitherto been discrissing are chiefly facts, measured more or less exactly by numbers and tables. The topic to which we now allude cannot be reduced under any formularies, and is indeed not easily rescued from those commonplace maxims which have currency in the world. That the passions and anxieties of life, if protracted or in excess; do more or less tend to shorten its duration, either by chronic impairment of health or by sudden injury to some vital organ, is a truth too well known to require argument. And deep though the interest of the subject is, we can give only a passing notice to it. 
The simple but certain fact is what we have already stated, viz. that the healthiness of the organs ministering to the several functions of life constitutes the health of the man; and in the same proportion tends to prolong his age. Though physiologists hold some verbal dispute on this point, yet can we hardly define Vitality otherwise than as a force or power, acting through the intervention of the nervous system upon organic structures, and depending upon the integrity of all these parts for its own amount and completeness. While admitting that the power is one which controls, and often seemingly contradicts, the physical laws most familiar to us, still we see that it is a power generated within the body; that matter and organisation are necessary to its action; and that by these it is variously and unceasingly altered through every part of individual existence. We are enabled, and indeed almost compelled, to speak of it as a quantity; varying in different individuals by original organisation;in each fluctuating continually during life; - and reduced to its minimum where life is prolonged into old age. What poetry has described as the blood in 'languid eddies loitering into phlegm,' may not be physiologically true; yet it is in some sort sanctioned by the doctrine of a great physiologist as to the especial vitality of this wonderful fluid; of which, even now, we do but partially know the physical properties and the changes it undergoes in health and in disease.

Carrying this view into the practical question how vitality may best be maintained and prolonged into old age, we must look mainly to four general conditions, which may be said to include all that is most essential to the fulfillment of the object. These are, air, as belonging to the function of respiration;-aliment;- exercise of the body; - and exercise of the mental functions.

The first of these topics - that of the air we breathe presents more difficulties than might be supposed; owing in 
no small part to the vague notions and prejudices current on the subject, and which science has hitherto but partially corrected. The contingencies of climate, for instance - whether hot or cold, wet or dry, equable or variable - are made the subject of current phrases, often unfounded in fact, and as often of injurious application. Since the Continent of Europe has been laid open to universal travel, local interests and fashions have tended further to distort the truth; and health is run after, whether from climate or mineral waters, upon the most ignorant plausibilities; and with little regard to other circumstances which often more than contravene the benefit sought for. The lungs may gain good from a warm atmosphere, but this may be paid for by gastric disorders scarcely less noxious. Heat is too commonly regarded as a panacea for all our bodily ills. In truth, cold has an equally fair title to take its place in the class of remedies; for in many cases where health is inertly loitered away under southern suns, the frame might have gained vigour and vitality among northern mountains. A comparison of the registers of mean mortality in these respective localities goes far to sanction this opinion.

But we must not deal with this subject as represented by climate only. The amount and purity of the air we breathe is a question belonging to every place, and of far more interest to the great mass of mankind. We do not here enter into the chemical theory of respiration, or the several controversies it has engendered. What concerns us is the fact, that a certain number of cubic inches of air taken into the lungs at each inspiration, and this air of a certain purity, are conditions necessary to the health and full vitality of the individual being. The imperfect attainment of these conditions throughout the whole, or a part of life, tends in the same proportion to enfeeble the vital power, and to abridge more or less the term of existence. The insufficient 
production and maintenance of this power impairs longevity, not less than its too lavish expenditure in the various abuses and vices of social life.

Hence the vast importance of all that may be done by open air, exercise, ventilation of dwellings, and removal of noxious exhalations, on behalf of this great function of life. These things are better understood than they were, and more is attempted and effected for their attainment. Medical science is now called upon to prevent disease, as well as to restore health. Much more might be accomplished in this way, as well for individuals as for communities, were the healthy state of respiration cultivated with the same care which is given to the actions of the stomach and alimentary organs. We doubt not, for instance, that this function is capable of being restored, improved, and maintained in efficiency, by well-ordered exercises of the lungs; and by due attention to the mechanism of these organs in all that belongs to the habits of life. It may not occur to our readers, and yet it is strictly true, that the familiar conditions of posture of body, speaking, singing, \&c., are deeply concerned in this matter; the more so from the very familiarity of every-day use. If forty cubic inches of air taken into the lungs at each inspiration are required for the purposes of their function, and thirty or twenty only are inspired, it is certain that the blood will not be duly changed, and that every organ and action of the body will suffer more or less by this deficiency. To enlarge the quantity then in such cases is an object of high importance; not sufficiently regarded, we may add, in the medical practice of our day.

Of the value to health of the purity of the air we breathe, it is hardly necessary to speak. Free ventilation is here the main agent in our hands, whether we look to the replacement of air despoiled of its oxygen, or to the removal of noxious matters present in it. Our actual knowledge, it must be 
admitted, does not enable us to exclude altogether those noxious ingredients (some of them doubtless animal or vegetable organisms) which produce epidemic and endemic diseases. Nor can we yet deal with those equally unknown influences on the body, for good or for ill, which depend on electrical states of atmosphere, the proportion of ozone, \&c. But science is now actively directed to these various objects; and meanwhile we may fairly assume change and freedom of air by ventilation to be the most efficient preventive means in our power.

Looking next to aliment in its connection with health, and therefore with longevity, we encounter a topic which has been endlessly discussed and written upon, and made the subject of various and conflicting opinions. All this is natural and inevitable. For the subject in question embraces not only what is necessary to human existence, but also what belongs to man's luxury and sensuality; and is, moreover, connected with all those changes of bodily condition, whether healthy or morbid in kind, which are most open to common observation. Including further those many forms of liquid, from simple water to the strongest alcoholic drinks, which the natural or perverted ingenuity of man has mingled with his aliment, it brings in another class of effects, of deep interest not only to individuals but to the welfare of social life.

Vague though it may seem, we can find no more fitting word than moderation, to express that which is best in diet in relation to health and length of life. No specification of the wholesome or unwholesome in food can be of avail for good, unless submitted to this one condition. As in the relation of the lungs to air, so the digestive organs require a certain quantity of food - varying in different individuals, and in the same person, at different periods - to maintain the healthy state and sufficiency of the blood, and through this 
the due action of the nervous system, and of all the organs of the body. And nature, where unspoiled by vitiated habits, furnishes a rule and measure which everyone, whatever the diversity of temperament, may safely and expediently, consult for himself. Here especially, however, men are more prone to be governed by faulty habits and injurious maxims, than to be convinced by reason or instructed by experience. That appetite, for which the old epicure would pay any price, is too much regarded as a condition to be instantly removed by food. Even under disease, when nature resumes her rights, and rejects with loathing the aliment the stomach has no power to digest, the mischievous zeal of friends comes in; feeding the disorder and not the body, by what they force upon the patient. The catalogue of ills which indigestion directly or indirectly involves - from the early oppression after food, to those later and more various effects, both on body and mind, for which the hesterna vitia are responsible, - might seem sufficient to enforce a rule, were they duly recognised in their origin. We may reasonably regret that the term dyspepsia, while actually expressing this origin, should in effect throw a sort of classic veil over the simple and certain source whence these ills arise. Common phrases are better suited to common things, and more salutary in their influence.

It has been a question mooted of old and often revived, whether intemperance in food, or in drinks, is most injurious to health and life. An eminent physician of antiquity, Celsus, pronounces against the former; and if the question be so put as to exclude the wilder forms of inebriety, we may perhaps acquiesce in this opinion. There is some risk, however, in discussing a matter of preference, where both contingencies are so prolific of evil. It is not uncommon to hear instances cited of prolonged life in drunkards, and doubtless such do occur. But they are as certainly ex- 
ceptional; and fairly subject in each case to the common and obvious explanation, that life would have been longer extended had the habit been otherwise. The drunkard who dies at 70 , might have reached 80 , if sober.

But while thus associating moderation of life with length of life, we are bound to add that there may be excess even in this laudable direction. A constant and anxious care for existence, and rigid rules of living founded upon this, tend in their own way to curtail what it is sought to prolong. There is some practical truth in the story in the Spectator, of the gentleman who cut short his life by weighing or measuring every article of his food. Of the famous case of Cornaro we have already spoken, as not justifying all that M. Flourens seeks to derive from it. The truth we consider to be - and it is a physiological fact - that whatever lears the mind to give close and minute attention to the stomach and organs of digestion, does more or less embarrass that great function, and impair the nutrition of the body. Temperance must not be made to march in manacles and fetters, or with steps of unbroken uniformity. We degrade the virtue by this manner of using it; and attempt what is impossible under the endless changes incident to the life of man.

It remains for us to speak of exercises of the body, in their reference to longevity. Under the definition of vitality already given, it will be obvious that whatever tends to maintain the organs generally in their healthy state, tends in the same proportion to preserve and prolong life. Exercise is one of the great means to this end. An organ destined to a particular function is best kept in its integrity by the exercise of this function, with a due regard to those progressive and inevitable changes which time brings upon every man. If we take the term exercise in its ordinary sense, as expressing the muscular actions of the body, the conclusion is the same; - rendered still more explicit by our knowledge of the 
influence of these actions in maintaining the vigour and equality of the circulation throughout the system, and very especially in the skin and extremities; thereby keeping all the organs in healthy balance and relation to each other. Many. organs - systems of organs they may even be called - make up the wonderful fabric of our physical being; the due balance among which (disturbed more or less in every form of disease) is at once the cause and the expression of bodily health. The changes fitting or necessary in the habits of exercise, as old age comes on, cannot be measured by years only. The time and the necessity vary for every individual; and must be determined for each by a just observation of himself. A wise man will find steps and stages in the descent of life on which to repose a while; without sudden or entire relinquishment of those bodily habits which, discreetly used, conduce to health and preservation at this period as well as in youth and middle age. The discretion needful is that suggested by experience. Whatever amount of exertion is sensibly felt to exhaust the bodily powers, in any of their functions, is fitly to be avoided. Whatever can be maintained without such effect is certainly safe and probably beneficial.

A story is current of Lord Mansfield, who himself lived to 89 , that whenever very aged witnesses appeared in the Court over which he so long presided, it was his wont to interrogate them as to their habits of life; and with the result that in no one habit was there any general concurrence, except that of early rising. Such anecdotes are for the most part either untrue or exaggerated. Early rising is doubtless in many ways favourable to health; but it cannot be taken as a guarantee for longevity. Even were the story in question true, it is more probable that the vigorous vitality in these instances maintained the habit, than that the habit alone maintained the vitality. 
What has just been said about exercises of the body in relation to longevity will, in great measure, apply to the mental functions also. We cannot indeed assume, because facts disprove it, that there is any exact parity between the mind and body in their connection with mere age. The mind may, and often does, retain its faculties little impaired, when vitality, as expressed by the bodily powers, is reduced to the lowest ebb. But let this reduction go further and they too give way; in obedience to the common law which the Creator has assigned to man on earth. The question before us regards the capacity for prolonging their duration, and the means conducive to this end. And here we come upon the track of a great writer, whose views on the moral and intellectual culture of old age, though somewhat florid in colouring, have always earned the respect due to his name and philosophy. The doctrine of Cicero is that the faculties of the mind in old age are best maintained by their exercise. 'Manent ingenia senibus, modo permaneant studium et industria.' In this doctrine, and on the same grounds just asserted as to the preservation of the bodily powers, we may fairly acquiesce. Every intellectual faculty is dulled and diminished by want of use. Each one is maintained in vigour, if not improved, by its fitting and temperate employment. This maxim, true generally to every time of life, requires no other modification for old age than the simple one of additional care that there be no habitual excess. Vitality is weakened or exhausted by intemperance of mind as well as of body; and in old age is less easily repaired. The brain, that organ which comes in such close and mysterious relation to the mental functions, more especially needs this forbearance in advanced life. At this period it readily becomes the subject of disordered action or disease from any excess of use, even of the intellectual powers; - much more still from any intemperance or disorder of the moral 
emotions. Quietude and forbearance form the right rule in our hands, and not premature disuse and abandonment of the faculties committed to us. These beyond doubt are better preserved by their exercise, within the limits we have just denoted.

Here again we have the authority of Cicero to refer to, and willingly adopt it. He gives us various instances of the exercise and preservation of the mental faculties to an extreme age; and such examples, in truth, are familiar at every period, and to the individual knowledge of us all. We might cite many that have come within our own experience. For the most part, it must be admitted, such cases as these are connected with a sound bodily organisation, concurrently preserved. But this, as we have already stated, is by no means uniformly the case. The disproportion of the two powers makes itself known to us in numberless instances. From the

Souls that can scarce ferment their mass of clay,

to that elsewhere described by the same great poet:-

A fiery soul that working out its way,

Fretted the pigmy body to decay,

And o'er informed the tenement of clay, -

we have every grade of relation between the two great faculties, which in their mysterious conjunction make up the nature of man. No more curious, but no more difficult part of human physiology than that of defining these relations, and the conditions which influence and alter them. Here it is that disease often serves us as the best interpreter; by detaching and insulating, as it were, different functions, which in health are so closely associated as to escape all division or definition.

The Memory is generally the mental faculty which is first 
and most obviously affected by old age. This wonderful intermedium between body and mind, varying so greatly in different individuals, and so strangely capricious in the same individual from the accidents of the day or hour, would seem to partake more of mere mechanism than any other of the intellectual powers. It undergoes changes more explicitly from physical causes; and both its excellences and defects are marked by peculiarities which appear to belong to conditions of organic kind. The anomalies of memory in advanced life are familiar to every one; especially so the fact of the early forgetfulness of names, and the frequent retention of things long past, while recent events flit away like shadows, leaving scarcely a trace behind. Or, more strangely still (though never perhaps without some morbid changes of brain), the obliteration of certain classes of events or certain subjects of memory, as if by a sort of mechanical separation from everything else abiding in this mysterious receptacle.

The importance of preserving memory in its integrity, as long and as far as it can be done, will probably be admitted. Some may urge that an oblivion of things past is the best security for a tranquil old age. But this virtually reduces man to a mere fraction of existence; and the same reasoning might be used to prove that utter imbecility of mind is a blessing in this latter stage of life. Such imbecility, from natural causes, often occurs; but we have no title to consider it a good, or to neglect any means which may obviate or retard it. We will not venture to say that these means are many or certain. As regards memory in particular, all that can be done at this period of life is to aid in giving it the direction which circumstances make desirable, and to spare it those painful efforts at recollection which seem to weaken the very faculty they exercise. The latter remark we believe to be of valuable application to other periods, 
long antecedent to old age; but especially perhaps to that time when the faculty is first felt to decline in clearness and power. Recollection (that is, the effort of the mind to combine or extricate what is laid up in the memory, the $a ̉ a^{\prime}-\mu \nu \eta \sigma \iota s$ of Aristotle) cannot be carried beyond a given point without begetting a certain confusion of mind, hurtful to the faculty itself, and probably to others also. The consciousness of everyone will give proof of this; and at the same time, if truly consulted, warning to avoid it.

We cannot close this article without adverting to that question, which at every period has been so variously agitated,- whether longevity be desirable, or not? A momentous enquiry indeed, if it really admitted of any determinate answer. But none such can be given. The conditions are far too complex to warrant any general conclusion; and even in individual cases, and with direct appeal to those concerned in the question, the difficulties are hardly overcome. The feelings of one moment change at the next. Even where their expression may be relied upon, longevity itself is a vague term; and rendered more so by the various contingencies of health and power preserved, which alone can give just measure of life or of the capacity to enjoy it. The old man of 80 , and he of 100 , may be on a par as to those conditions upon which we found our only valid estimate for each.

We must then receive with some allowance those writings, eloquent though they may be, in which the cause of old age, as such, is pleaded before us. Here the name and authority of Cicero again come into view. Though we are unable, with Montaigne, to say of his treatise 'De Senectute,' 'il donne l'appétit de vieillir', we can well admire the fervour with which he maintains his thesis, and the happy ingenuity of his argument. Nor can we grudge him his eloquent 
abstraction of what old age might be; while admitting that, however rare and difficult their attainment, the objects and methods he indicates are all fitted to give honour, tranquillity, and usefulness to this stage of life. Add to these the religious confidence, which Cicero could only vaguely, if at all, proffer, and we have a summary of whatever is in man's power towards the attainment of that worthy object, a happy and venerated old age.

But, to reach this end, the preparation must be begun long before. Without infringing too far on the style of the pulpit, we may point out the main fact that the habits, feelings, and interests of earlier life are all carried forward into old age; and often intensified in degree, by the removal of the circumstances which before tempered or constrained them. 'On ne jette point l'ancre dans le fleuve de la vie,' is the happy phrase of an old French writer for that continuity of life, by which all its parts are linked together; and the young man, in his intellectual, moral, and physical habits, becomes the interpreter, more or less, of what follows in his after-age. When Lord Bacon says, with his wonted weight of words, 'Strength of nature in youth passeth over many excesses, which are owing a man till he is old,' he expresses a physical as well as a moral fact, which cannot be too well weighed in the education and conduct of early life. It is a maxim full of practical wisdom.

We have already alluded to the various sentiments with which old age, and approaching end, are regarded by the aged themselves. In many of them the desire to pass away, and this even without the solicitation of active pain or suffering, is equally earnest and sincere. It is with them as with the 'Tre vecchi' in the Purgatorio of Dante:-

E par lor tardi

Che Dio in miglior víta li ripogna. 
Individual temperament is partly concerned in producing this weariness of protracted life; but other causes also come into operation to which we have not space to advert. There is one fact, however, which we may briefly notice, inasmuch as it seems a providential dispensation to the latter stages of existence; and we do so by borrowing a few lines, which succinctly express the circumstance to which we allude.

No previous reason or feeling, no judgment of vigorous health, can afford a right estimate of the relation the mind assumes to death in the latter hours of life, even where little impairment of its faculties has occurred. This is especially true where long and painful sickness has been the prelude to the event. But the exhaustion even from acute pain of short continuance alters this relation; and even without sickness or suffering of any kind, the mere diminution of vital power by general decay produces the same effect. The earnestness to live abates, as the possession of life, from whatever cause, is gradually withdrawn.*

This, we think, will be recognised as true by those who have been observant of these things; and witnessed the changes which gradually supervene on the feelings, as the physical conditions of vitality abate in power, and action subsides into repose. We willingly close our observations at this point. If unable to assent to the doctrine of M. Flourens that a century is the natural term of human longevity, we thoroughly agree with him that individual habits may be made to contribute much to the healthy prolongation of life; and we can affirm with assurance that these habits are such as best accord with the happiness, dignity, and higher destinies of our species.

* Medical Notes and Reflections, 'On the Medical Treatment of Old Age.' 


\section{ROMAN HISTORY - JULIUS CASAR.*}

[Quarterly ReView, March, 1851.]

GTRANGE though the fact may seem, at a time when 1 learned and ingenious men are seeking subjects throughout every domain of human knowledge, it is certain that we have no English work, deserving the name of a history of the Roman Empire, prior to the point at which Gibbon takes up his vast and splendid theme. Nay, this deficiency, it can hardly be denied, extends over much of the antecedent period. It might fairly be deemed a vacant field to which Dr. Arnold came, when he undertook the work which was abruptly and unhappily terminated by his death. His learning and candour fitted him well for the task; and though there are some defects of method in its earlier part, no writer need disdain the task of completing what he has thus begun. Such completion is imperatively required to sustain the fair fame of our literature; so faulty on this subject, that even now it is difficult to place before the student any English book which creditably relates the great events intervening between the close of the second Carthaginian war and the death of Sylla. $\dagger$ The work of Middleton

* A History of the Romans under the Empire. By Charles Merivale, B.D., late Fellow of St. John's College, Cambridge. 2 vols. 8vo. 1850.

Feb. 1862. The seventh and concluding volume of Mr. Merivale's History is now, we believe, on the point of publication. The work thus completed, forms a most important and valuable addition to English historical literature.

$\uparrow$ The valuable History of Rome by the present Dean of Christchurch had not been published when this article was written. 
comes in at this time; but owes its reputation much less to its own merits or originality than to our deep interest in the actions it records, and to a comparison with the bald and feeble essays which precede it; - volumes uninformed by critical research, and destitute of every charm which style or philosophy can afford.

Our continental neighbours have dealt more copiously and successfully with this great subject. In France the writings of De Beaufort opened that vein of sceptical enquiry as to the early history of Rome, which has since been so boldly pursued elsewhere. To the theme of her grandeur and decay Montesquieu brought his high philosophy; Vertot, Michelet, Thierry, \&c., have furnished works well fitted for study; while other writers have diligently pursued those researches which connect the Roman Empire in Western Europe with institutions and usages existing down to our own times.

The German scholars of the last half century have given to the Roman History that zealous and minute labour which is their great characteristic. It may well be called an exhaustive power of research, for they leave no record or fact untouched; though often, it must be owned, without a sufficient regard to the authority or worth of the materials they accumulate. They have taken up this subject, like others, not merely on the broad scale of history, but in detached parts; illustrating more fully the course and effect of certain political changes, and the career of those men whose genius or fortune have urged on such revolutions. We may notice as examples Schulze's history of the Republic to the time of the first plebeian consul; Hegewisch's and Heeren's history of the Gracchi; Heyne on the social war; Schleuter's history of the period between the two narratives of Sallust; Meissner's life of Julius Cæsar. The more recent work of Drumann well deserves the euolgiums Mr. Merivale has bestowed upon it, and the use he has made of it in his 
own. Nor can we omit mentioning the Stemmata Gentium Romanorum (the genealogy of Roman families) of Ruperti, as one of the most valuable aids to Roman history yet published. Finally, we must name the great work of Niebuhr; coldly or harshly critical it may be; often theoretical; and in parts doubtful or mistaken; yet correcting much of common error, and affording a juster estimate of the relative value of those documents, whether Greek or Latin, from which the Roman history is derived.*

Mr. Merivale now aspires to fill the historical void in our own literature. We opened his book with interest, but not without jealousy; because, even if it had not worthily fulfilled the objects designed, it might yet have so far succeeded as to deter others of higher pretension and literary power from attempting the same labour. This is a space in the world's history far too vast and important to be allotted to anyone who is unable to found thereon a fair and lasting edifice. Mr. Merivale, however, was already known as a very accomplished scholar; his reading and power of Latin versification had been placed advantageously before the public; while his 'Age of Augustus,' published a few years ago, was a natural antecedent, and to his readers a full justification of the present undertaking. Whether he regarded it as tentative of this larger work, or was led to the latter by getting thus far into the subject, he does not inform us. But we can well understand that a writer who found himself immersed in the epoch of final change from republic to monarchy, might naturally proceed to spread his scheme over the first great period of the Empire.

Mr. Merivale could not have dispensed with a preliminary

* Since this article was written, the volumes of Sir George Lewis, on the 'Credibility of Early Roman History,' hare shown what may be done by an accomplished scholar, of equal industry and learning, even amidst the labours of high official life. 
outline of Roman history, even from its origin. In any case, to render such a summary clear, just, and effective for its purpose, is the highest test to which an historian can be put. In the case of Rome, the difficulty exceeds perhaps that of any other. We think ourselves familiar with its history from the teaching of schools; though this knowledge is usually but of events only. Few comprehend at all distinctly the strangely interwoven elements of Roman government and internal polity; the progressive changes therein; the mutual effects of these changes; the influence of foreign conquest on the social and political condition of the State; or those other more secret and subtle causes which are ever at work, altering or undermining all human institutions. If the reader has at any period devoted himself to such studies, the summary in the first of these chapters may be sufficient to refresh his memory of all that is most essential. But we have some doubts whether it will adequately instruct those who come only half informed to the subject, and for whom it is the duty as well as profit of the historian to smoothen the road to the threshold of his work.

Mr. Merivale opens his volume with a somewhat ambitious description of the topography of the Seven Hills; and depictures the isolation and fierceness of the Roman character, as connected with the solitary wildness of this locality. 'Such a position,' he says, 'was admirably adapted for a place of retreat, and offered an impregnable shelter to crime and rapine. It seemed created by Nature herself to be the stronghold of a people of reserved character and predatory habits. It was destined to become the den of the wolves of Italy.' Our author is hardly justified in thus describing the early Romans by the phrase of a defeated enemy. Nor have we much faith in the inferences here drawn. The Seven Hills, even if they offered shelter to the infant city, did not nurture it into greatness. They owe everything to Rome- 
not Rome to them. A hundred localities of the same land would have offered like advantages. The soul of Roman greatness was not in the shelter of its hills, but in its civil and military institutions, and in the unity of spirit and vigour of action they engendered; which kept the State from being ever confederate with others, save when she became their conqueror and chief.

The growth of a single town, small and obscure in its origin, into the empire of the then known world, is a prodigious phenomenon. We need thus to bring together the two extremes, before we can fully comprehend how greatly the fact surpasses any kindred event in the history of nations. And even in the decline and fall of this vast fabric of power we have fresh cause for wonder at the slender foundation of an edifice, stable enough to resist so many centuries of decay, and leaving such ample vestiges to later ages. Fortuitous causes are out of the question; nor is any theory of race or temperament more admissible. Whether we consider the founders as a colony, or as a band of lawless adventurers, which Mr. Merivale seems to suppose, equally certain is it that they were of the same Latin stock as other neighbouring tribes; - modified, it may be, by intermixture, or other incidents to which such small communities are liable. Throwing aside what is poetic and legendary in the history of Rome, we cannot look elsewhere than to moral causes for its grandeur of growth. Partially modelled under the rule of the kings; - more largely evolved in the change to republican government; - farther extended and matured by those internal struggles of classes which more than once threatened the existence of the State - the institutions of Rome survived in show when their virtue was extinct, and lent a specious shelter even to those usurpations which converted a republic into an empire. The outward forms of the Roman Senate were continued as matter of policy, 
long after their real grandeur and political efficiency had passed away.

The living energy these institutions possessed in the better times of the Republic has no parallel in history, ancient or modern. The harsh and limited character of Spartan institutions removes them from the comparison. Athens, even in the utmost vigour of that democracy which so deeply engages Mr. Grote's admiration, did not put forth the unity of action, or create that passionate devotion of citizens to their country, which is so striking in the annals of Rome. The internal polity of Venice was too complex and corrupt, even in its best days, to admit of its being brought into the parallel. With every allowance for that various casualty of events, to which nations as well as men are liable, it is impossible not to see in the fate and fortunes of Rome how much her constitution rose above others in solidity and active power. The successive and fierce struggles of the plebeians for a guarantee of personal liberty, for division of lands, and for equal right to all the offices and rewards of the commonwealth, show how deep the foundation was laid; these contests actually invigorating the State which at the moment they seemed fated to ruin. The expression of Florus, Magna populi Romani fortuna, sed semper in malis major resurrexit, applies as well to the recovery from civil dissensions as to her proud survival of the foreign assaults which repeatedly menaced her existence.

Though the greatness of Rome could not have arisen from fortuitous causes, yet we must admit that the adoption and growth of the institutions which engendered it may have resulted much more from what we call accident than from actual design. In truth, no human intention could have produced such a fabric, any more than it could have created the complex structure of our English constitution. We have every reason to suppose that Rome adopted into her primi- 
tive government many usages from the Latin and Etruscan states. These were progressively moulded and modified as with us; - in some cases by convenience or necessity, in others by the direct collision of different influences and classes. In both instances the result may in part be attributed to the comparative insulation from neighbouring states. While the Etruscan and Latin cities were engaged in confederacies, more or less extensive and binding, Rome was almost always single in her course of action. Her connections with other states were mainly those of conquest and supremacy. Her institutions, whether of peace or war, all appertained to the City itself. Her rights of citizenship, even when most extender by prudence or necessity, flowed from within to without. Her colonies, unlike those of the Greeks, never assumed the condition of independence. Her most distant wars were conducted, her most distant provinces ruled, by men chosen within the walls. The forms and superstitions of the national religion were maintained wherever her arms or her magistracy were present. Conditions like these, however originating, could not exist without large influence on the destinies of a state. Yet the greatness thereby created had in it a germ of decay, derived from those very elements of power, and growing with their operation.

In one of Hume's essays he mentions three anomalies of government as among the most singular which history affords - the $\gamma \rho a \phi \eta \pi a \rho a \nu \rho \mu \omega \nu$ of Athens, the relation of the Comitia Centuriata and Comitia Tributa of Rome, and the impressment of seamen in England. The second of these is, indeed, a striking instance of the peculiarity and integrity of Roman institutions. Here were two distinct legislative bodies; opposed to each other in origin, interests, and manner of action; yet, amidst all the civil contests in Rome, the only instance of actual collision between them is one recorded by Appian, trivial in itself, and happening at a time 
when the Republic was well nigh extinct. No one beforehand could have predicted such a result. No state has afforded a similar example.

The office of Dictator, in itself almost as great an anomaly, must be counted among the most admirable of the Roman institutions. Based on the principle of preservation of the commonwealth, it rested for its action on public virtue and obedience to law; and it is wonderful for how long a period, whenever the agency was invoked, this confidence was justified by the result. Some partial analogies to the office might be found elsewhere; but none approaching it in grandeur and efficacy. It is purely Roman in its every part; - in the choice of men; - in the limitation as to time; - in the frequent surrender of the dignity the moment its object was fulfilled; - and even in the law, trivial as it may seem, which forbade the supreme functionary to appear on horseback without permission of the people. A void of more than a century had occurred in the Dictatorship when Sylla assumed it. But it was the name only, without the ancient virtue of the office. The Dictator was now the military tyrant of the State, no longer the guardian of its safety and freedom. The fact is one which well illustrates the mighty change that had already taken place in the social and political condition of Rome.

The author who is about to record the change and decay of the Roman constitution ought clearly to expound this constitution in its nascent and progressive state. Mr. Merivale has given a rapid but faithful summary of the struggles on the agrarian laws; - of the momentous changes which raised the plebs to equality of privilege with the populus; - of the principle of Roman colonisation; - and of the contests and concessions by which the citizenship of Rome was finally extended to all the Italian states. But he says too little respecting the origin of the Roman people, or of those forms 
of government which conduced to their greatness; - and alludes to the peculiar isolation of the Republic rather as a proof of their barbarous character than, as we have found cause to consider it, an element and cause of their progress and persistent success. He speaks of them indeed as the normal type of a conquering race; but very slightly shows whence this type arose, or in what it consisted.

$\mathrm{He}$ has justly and vividly depicted the increasing and already intolerable corruption of Rome at the time when his narrative opens. Many causes contributed to this; - none more than the system of provincial rule. Consulships, and other curule offices were sought for, not from regard to the public weal, but as steps to the government of those provinces which yielded most ample spoil. No sovereignty so harsh or destructive as that of a Roman proconsul, fostering his private vices, or forwarding the projects of his future ambition, by the riches torn from his temporary subjects. The recorded wealth of Crassus was less nefariously acquired; but the high position it gave in the commonwealth to a man of his slender merit, strikingly illustrates the change of manners that had taken place. The growth of the City, increasing with that of dominion and citizenship, brought together a base and brute multitude, alien to the glories of Rome, and ignorant of the better institutions of the ancient time; ready indeed to render service to any Catiline or Clodius who might call them to the work of revolution and plunder. These abuses had sunk so deeply into the morals and discipline of the Republic, that the restoration of primitive ideas and usages became impossible. It would have taken a whole generation of Catos to accomplish it; and of Catos more truly wise and practical than he who has carried the name to posterity. The institutions and virtues of Rome had alike decayed; and that decay was not the simple decrepitude of age, but the worser disease of human passions let loose by prosperity and 
power. Both within the City and without, save among a few old republican enthusiasts, there existed the feeling that a change was at hand - inevitable if not desirable.

The epoch of Marius and Sylla; and the civil war begotten by their ambition and jealousy, form a threshold to the events which occupy the still more remarkable period before us. These extraordinary men, each victorious over foreign enemies, each taking the badge of a party and contending fiercely for superiority at home, did much to hasten the catastrophe in progress. Marius - an admirable soldier, but coldly and brutally unprincipled - is readily understood. He assumed the cause of the Italian states against the ruling aristocracy of Rome, from the personal motives just named, and to recruit the armies which subserved to these objects. He probably had no real purpose beyond, though party spirit gave him credit for such. Sylla was of higher stamp; one of those who stand out in bold relief on the world's history; great in intellect, constant in purpose, intrepid and powerful in action; but blasted in his moral part by a contemptuous indifference to human life, virtue, and happiness, which led to the perpetration or permission of cruelties, hateful in their very record. It needs a large view of the contradictions of human character to explain the anomalies of this man's mind and career. It is usual to speak of him as the champion of the nobility and old senatorial families against the encroachments of democracy within the City, and the pressure of the new citizenship created without: and such undoubtedly he was in the changes he effected during the two years of his dictatorship. But we stop short of believing, as some do, that his single and settled purpose was that of restoring the integrity of the Republic. Personal passions mingled themselves with, if they did not decide, his public acts. An early hatred of his rugged rival Marius was embittered by time, and by the cruelties of their protracted con- 
flict. Ambition had doubtless also a large share in moulding the events of his life. Such a man, so gifted with the ability for power, could not do otherwise than grasp at it. His sudden abdication of what had been won at such cost of toil and blood, is not so incompatible with this as it might seem. We dare not set down anything to principle; but neither can we admit the notion of fear as prompting the act. We look rather to the pride of a man who had nothing further to gain; to his contempt of the world he had thus mastered, and to his love of luxurious indulgence, as it is described to us by the writers of the age. Or it might be, that he already felt the approaches of the disease, whatever this was, which shortly after ended his days. The annalist easily records that event which stops alike the career of all - of the great, the brave, and the wise - but he is ignorant, or takes little note, of those more subtle causes, which though not sufficient to stop the current of life, yet check and turn it aside in its course; - of those physical changes which put passions to sleep, and paralyse the powers of action. History revels in tales of poison and secret assassination, but is silent as to the secret disease of organs - the slow poison of bodily decay. Yet it is certain that these things are deeply concerned in worldly affairs; and we could suggest many cases of historical paradox, which may be best solved by looking to them alone. Such documents, however, are written for the most part in too delicate a character to be legible by the historian; and we must needs be content with, and give what credit we may, to the coarser materials which are put in evidence before us.*

Again, there is an obvious facility in defining character by strong and arbitrary lines; and there may often be a moral

* Since this article was first published, two biographies of Sylla have appeared in Germany, by Drs. Zacharia and Lau, manifesting the usual industry of German research. 
use in this, inasmuch as the highest grades of virtue and excellence are those which best will bear such definition. But the author who generalises too much in this matter invents a drama rather than writes a history; and his personages become puppets, moved by his own hands, not the real actors on the great stage of the world. Though it be true that every man has a certain mental and moral temperament from his birth, more or less apparent throughout life, yet is human character, in the common sense of the term, made up of too many elements often strangely incongruous in themselves, to be submitted to any standard of unity. Accidents and conversions interpose in this as in all other human things; and it would be hardly less an error to attribute all events to a blind fate than to assign them universally to fixities of purpose in the agents. The consciousness of every man tells him of such alternations and anomalies in himself. The appeal from Philip drunk to Philip sober has a meaning beyond the mere anecdote; and the noble poetry of Dryden is true to life in picturing, under another influence, the rapid changes of mood and mind in Philip's greater son. We advert to this matter, not as a mere contingency of error, but as an actual fault in historians;- $\mathbf{a}$ fault which it is the more needful to guard against at a time when fiction, open or concealed, presses so hard in various ways on the true history of mankind.

Scarcely had the troubled period of Marius and Sylla come to a close, when there sprang up the concurrence of four wars; - that with Mithridates in the East; the vigorous struggle of Spain under Sertorius in the West; the devastating war of the pirates in the Mediterranean; and that of the gladiators in the very centre of Roman power; - contests formidable separately; - capable perhaps of subverting the Republic, could they have coalesced in action as they coincided in time. These wars, whatever their effect on the fortunes of 
Rome, gave lustre to the name of one great commander, and raised him to high influence in the State, strengthened by his relation to the ruling aristocracy of the City. The yet greater rival of Cneius Pompeius had no such early harvest of glory. Though his descent was ancient and distinguished, and his talents always conspicuous, yet was he chiefly known during the first period of his life in city profligacy or party conflicts ; and gave little certain augury of that wonderful career of victory which made him master of the Roman world, and rendered even his assassination ineffectual to annul the sovereignty he had created.

Our author's second and third chapters are chiefly occupied with the conspiracy of Catiline, and with sketches of the character and early life of the two illustrious rivals just noticed, and of others who played an eminent part in the great drama of Roman revolution now approaching to its crisis. Of what relates to Julius Cæsar we shall have occasion to speak afterwards. As respects Pompeius - (or we may be pardoned for adhering to Pompey as the name naturalised with us) the estimate Mr. Merivale forms of his character and public conduct is probably just on the whole; though we think he commits the error of defining too absolutely the course of thought and policy which led to his public career. It may be that Pompey saw and felt what our author affirms he did; but there are several circumstances which inspire great doubt on the subject. Energetic and successful in military action, his political course, where not actually feeble, was tortuous and uncertain even to his friends and confederates. Adopted the successor to Sylla as leader of the aristocratic party, he was often lukewarm, sometimes a traitor to their interests. His accession to the triumvirate comes closely under the latter interpretation. His permission of the violent and flagitious acts of Clodius when he might have prevented them, can hardly be explained, still less vindicated; and his 
relations, political and personal, to Cicero aggravate this charge against him. Warm and amiable generally in his private affections, he wanted the vigorous consistency needful to his ambition - more urgently needful when engaged in competition with a Cæsar. He brought to this conflict for the mastery of Rome the fame of his former acts and the support of the old nobility; for whom, though with a cunfidence abated by time, he was the only hope. Cæsar came to it, armed with present glory, and with a steadiness of purpose and action all his own. So confronted, it could not be doubtful how the contest between these two great Romans would end.

These views of the character of Pompey, and the doubt whether he held any settled scheme of political action, are mainly derived from the writings of Cicero; his advocate, as far as circumstances made it possible to be so - an advocate, or an accuser, not merely with his own time, but with all succeeding ages! In the case of this eminent man, also, a bold and skillful pen is wanted to serve the cause of strict historical truth, without needlessly offending opinions which have gained a sanction from the general adoption of posterity. The character of Cicero, as drawn by our author, is not altogether such a picture as might have been desired: neither his merits nor his foibles are brought out with sufficient force. Little is said of the consummate grandeur and completeness of his oratory, though upon these performances his glory mainly rests. His philosophical and purely literary works hardly add to his real fame, though they do not deduct from it. His epistles, admirable as documents of character and manners, are so at the cost of his personal reputation. Vanity, pedantry, feebleness of will, and feebleness of endurance, all stand in record against him under the unconscious testimony of his own pen. Such is the evidence, that we are forced, despite ourselves, to apply it to the greatest 
act of his public life, and to doubt whether his conduct in the Catiline conspiracy was all that he himself has depicted it to be. This doubt is strengthened from other historical sources; and the acclamation which hailed him 'Father of his Country' was probably a cry of momentary impulse, which a year later dwelt in few memories but his own. He met his death, indeed, with fortitude; but even here we have it from a high contemporary authority that 'it was the sole calamity which he bore as it became a man to do.'

Incomparable as an advocate, these other and lower qualities, and a certain jealousy as to his origin, forbade his ever attaining the highest position as a statesman, especially at the time of revolution in which his lot was cast. We have various proofs that Cæsar and Pompey thoroughly understood all his foibles, and worked upon them for their own purposes. To the masculine vigour and singleness of Cæsar's mind, in particular, they appear in remarkable contrast, and there is curious evidence how much the orator stood in awe of the great commander even before his career of victory had begun. We can well believe that the latter must often have smiled at the mixed humility and vanity of Cicero's communications with him; - the submissiveness of a conscious inferiority in will and action - the vanity of a man whom it is painful to call a pedant, but who in reality was such. In the midst of Cæsar's last Spanish campaign, one of the most critical of his life, Cicero introduced to him a young man, named Præcilius, in a letter interlarded as thickly with Greek phrases and quotations as is a modern fashionable novel with French; and, it must needs be added, with as little pertinency or fitness. It is true that he calls it genus novum litterarum; but still we feel it strange that such a letter should have been written by Cicero, and addressed to Cæsar.

There is something of moral wrong in indiscriminate praise as in indiscriminate censure. To this further reproach we 
fear that Cicero must be submitted. He was $\delta \varepsilon \iota \nu o s ~ \varepsilon \pi a \iota \nu \varepsilon \tau \eta s$ in the strongest sense of the phrase. His speeches against Verres, Catiline, and Antony, show how large an armoury of caustic language he had at command. But in his epistles and elsewhere, we find the most copious collection of laudatory terms in existence-one, indeed, that has served as a lexicon to the learned flatterers of every later time It is impossible not to see that he generally praises with a reflex view towards himself. He is governed much more by the seduction of his own style than by the reality before him. If the letters of introduction, of which he is so liberal, were but half true as to the virtues of those recommended, Rome could not have been so speedily submitted to the servitude which now hung over her.

The character of Cato is not formally brought forward by our author among those of the other great actors of the time. This we regard as an omission. He is one of those personages in history who have become, in some degree, the property of the poet and the moralist, and respecting whom there is a conventional language of panegyric not wholly in accordance with the rough and rude reality. The succeeding part of Mr. Merivale's narrative, in as far as it relates to the Roman Stoic, shows what the truth of history requires to be deducted from common repute regarding him.

The account of the intrigues and combinations which produced the first Triumvirate is clear and forcible. It was an unprincipled cabal, annulling by a transient union the real powers of the constitution, while keeping up its outward forms. The interests of the senate and nobles were sacrificed by one triumvir; those of the people by another; while the third ministered to the alliance that power which wealth gives in a corrupted state. What individual ambition could not yet effect, was attained by this conjunction. It was the empire of Augustus by anticipation, and conducting to this as 
a natural result. But it wanted that stability which unity of person and purpose alone afford, and was dissolved by the separation of the same ambitious interests which had created it.

The first effect of the Triumvirate was to give to Cæsar the Consulship, which he could not otherwise have obtained. He was regarded by the aristocracy of Rome as too dangerous a representative of the doctrines and acts of the Gracchi and Marius to admit of their acquiescence in the power which this office conferred on him. But the power was got; - the colleague whom they thrust in to cripple it thrown violently aside; - and the position of Cæsar further confirmed by the marriage of Pompey with his daughter. The uxorious temperament of the latter offered a pledge and security to Cæasar, during the long absence from Rome which was close at hand as the first act in his higher career. This career now lies before us in a more definite form than heretofore; and, though more or less familiar to all, yet, considering the grandeur of the man, the greatness of what he accomplished, and the influence this has had upon all succeeding ages, we may be excused for dwelling at some length on the subject. It occupies, indeed, more than one half of the volumes before us; and we cannot hesitate in admitting that Mr. Merivale has done it full justice. He rises in vigour as he gets free from the complex intrigues of the city, and embodies in his narrative that series of stirring events which carried Cæsar to single supremacy. To these events we would now seek to direct attention.

We have before noticed several foreign works, and particularly those of Meissner and Drumann, in which the life, character, and policy of Julius Cæsar are fully and ably handled. In England we are chiefly indebted to Dr. Arnold and to Mr. Long for what we possess on this subject; and in 
Mr. Merivale's preface he warmly and gracefully acknowledges the aid he has derived from the writings of the former on the later commonwealth of Rome. Of the original materials for the life of Cæsar, we have little room and not much occasion to speak. They are well known to scholars in their different degrees of value and authenticity. We may well regret here (as so often elsewhere), the lost books of Livy, whose personal knowledge of those who had witnessed or partaken in the acts of this eventful period, would have given still deeper interest and charm to his narrative power. We should willingly recover from the spoils of time the history of Asinius Pollio, the cynical companion of Cæsar in all his most arduous campaigns; and the letters and biography of Atticus, the tranquil observer and common friend of all parties, even when factions were fiercest. Yet more should we wish that the stern truth and lofty moral dignity of Tacitus could have been applied to the life of a man who made such mighty changes in the destinies of his country. These are vain aspirations; yet in some sort forced upon us when disheartened by the disputable stories of Suetonius, Plutarch, Dion Cassius, and other anecdote-mongers of antiquity. The authority of Appian is abated by distance of time and other doubts as to his histories. The little we have from Sallust upon this period the bias of the writer compels us to receive with caution. The Pharsalia of Lucan may not safely be taken as more than subsidiary authority to facts recorded elsewhere; though we are unwilling to utter anything in depreciation of this fine composition, which we can hardly agree with Quintilian in regarding rather as oratory than poetry. The materials which come to us for the life of Cæsar most free from cavil and doubt are his own Commentaries, and Cicero's Epistles and Orations. The former, whatever their merits, cannot be rescued altogether from the charge of partial representation. The latter need to be read with a critical eye, from the pecu- 
liarities of Cicero's character, and his political position in regard to the great men who figure in the events before us.

The early life of Cæsar affords two or three anecdotes which we cannot well distrust, seeing how entirely they accord with his later acts. His bold and successful collision with Sylla, then in the fullness of power and enforcing his will with blood,- and the chivalrous transaction with the Cilician pirates, - are instances of the strong determination, self-confidence, and personal intrepidity so amply shown in the sequel. The moral courage of the youth is said to have drawn a prediction from Sylla of the future fortune of the man. Such stories are often begotten by the event; but we can easily believe that Sylla might discover in a character having so much kindred with his own, those elements which are sure to be effective in a State bordering on dissolution.

The other information we possess as to the early part of Cæsar's life, with the exception of his study of rhetoric at Rhodes, places him before us as a reckless spendthrift, a city voluptuary, a fearless politician and partisan. His relationship to Marius gave name and foundation to a course of action which he would probably have pursued had no such connection existed. For though, in this instance also, we think Mr. Merivale too decided in assigning motives and method to political conduct, yet we cannot doubt that Cæsar, conscious of and confiding in his own powers, and observant of the decay of ancient institutions and ancient republican virtues around him, must have felt that a great arena was open to the exercise of these powers, and to the ambition which their possession was sure to inspire. Under such impressions he took the line of party most natural to him as the nephew of Marius, and offering a surer road to influence than the adhesion to a jealous, intriguing, and tottering aristocracy. Without pretending to affirm it, we see no cause to suppose more of scheme or foresight than this in Cæsar's 
early public life. He flung himself upon the tide of events then rushing stormily on - prepared to stem it with strong arm and heart of controversy - but yet unaware how he should be carried forwards, or on what shore his fortune would cast him.

This broad view tallies better, we think, with Cæsar's character and the records of his early life, than any more refined speculation as to his political and personal objects at this period. The juvenile excesses related of him were due in some part, probably, to physical constitution - an element never to be disregarded in forming such estimates; - in part, perhaps, to the desire of warding off suspicion at a time when the hand of power was strong against his party. We have already had occasion to comment on the frequent error of historians in regarding character as single and unchangeable, and parcelling out their theory of motives and events accordingly. The mind of Cæsar was as entirely individual, as little touched by time or changed by circumstances, as any on record. But it is perfectly consistent with this to suppose that his views were enlarged, and their direction determined by events themselves. The ambition with which he was early charged, he undoubtedly had; seconded by a strong and consistent will and high intellectual power; - and these qualities sufficiently defined his course in the existing state of Rome. He seems to have avoided any direct connection with the profligate plots so frequent at this period. We doubt his being otherwise concerned in that of Catiline than as a too indulgent spectator of scenes which might open new avenues to his own ambition. During the career of Clodius he was absent from the city; but he signalised himself by his efforts to shelter his political adversary, Cicero, whom Pompey, professedly a friend, betrayed to the violent demagogue. His own measures in the popular cause, both before and during his consulship, appear to have been in themselves 
neither intemperate nor unreasonable. His period of government in Spain was successful in arms, able in administration. But this was his sole independent command before the Gallic war; and when we compare his early course with the wide career and large renown of his rival, yet find them equally associated in the Triumvirate, we see that Rome had already learnt to know the loftier character and higher resources of Cæsar, and that this position was one which could not safely be denied to him.

In his fifth chapter, as an introduction to the Gallic campaigns of Cæsar, Mr. Merivale gives an able and lucid history of the great Celtic race, which in its different branches and at successive times came into urgent collision with Rome; once putting her very existence at stake, and often inflicting panic by the conjunction of these northern hordes with the Italian states hostile to the Republic. The last great alarm from this people had been the irruption of the Cimbri, in transient connection with certain Teutonic tribes, into Italy and Southern Gaul; - a gigantic armed migration, which swept away more than one Roman army, and required the strenuous arm of Marius to arrest it. Bloody victories, ending in massacres, satisfied the dignity and restored the safety of Rome. It was reserved for the greater nephew of Marius to complete the work on the soil of Gaul itself, and by the conquests of successive campaigns to bring the whole of this warlike country in subjection to the Roman power;a splendid achievement, and, from the causes just mentioned, duly estimated at Rome. The formidable king of Pontus, a worthy rival in arms to Sylla and Pompey, had disturbed only the distant possessions of the Republic. The Gauls once reached the Capitol, and still stood at the mountain gates of Italy, menacing her provinces, and requiring the constant watchfulness of her legions.

These Gallic campaigns of Cæsar, extended through his 
long proconsulship of nine years, splendidly illustrate the genius and resources of the man; and throw, moreover, a curious light on the still remaining institutions of the Republic. As proconsul, and with provinces and legions allotted to him, the law forbad his going out of the limits thus assigned. The City was interdicted to him; and for this long period of time - though forty years of age when this portion of his career began - he never entered the place in which the interests of the world were concentered. Yet in no other way could his ambition have been better served. The active part of each year was passed with his legions in marches and victories, and in the acquisition of spoils, with which to purchase further power. Leaving his army under his lieutenants at the end of the campaign, to be recruited and refreshed, he came himself each winter to the frontier of his province nearest to Rome, where he was met by his numeroùs friends and partisans from the city, animated by his conquests and increasing fame. His military court there had more validity in it than the habitual presence of his rival in the heart of Rome. It now became a contest between living success on the one side, and the memory of past achievements on the other - a contest which the world will ever decide in the same way:-

To have done is to hang Quite out of fashion, like a rusty mail In monumental mockery.

The resistance of the Gauls might possibly have been prolonged, or more successful, had they been familiar with the methods of guerilla warfare. But either from temperament or national custom, they aggregated themselves into masses wholly incapable of withstanding the organised valour of the invaders. The history of the tenth legion is familiar to every reader of these stirring campaigns. Cæsar was well 
served by his generals as by his soldiers. Yet, as in the case of Napoleon's marshals, their fame was little more than the reflection of his. Labienus, the most noted of them, deserted the standard of Cæsar at the time he passed the Rubicon; an act which served but to show how entirely it was the spirit of the great commander which ruled and invigorated his army. Though Labienus served the enemies of Cæsar until he perished in the battle of Munda, his name never again appears before us associated with any great action of war. Other causes may be conceived for this; but the main one doubtless was, the absence of that inspiration which came from the genius of Cæesar.

Our author's account of these military transactions is spirited, and fairly illustrated by reference to existing localities; though such is the life which illustrations of this kind impart that we could have wished them carried still much farther. We draw our chief acquaintance with the Gallic wars, it is needless to say, from the books which have come down to us under the name of the great chief himself. His Commentaries have ever held a high place among historical records; and no wonder, considering his fame, the masculine simplicity of his writings, and the greatness of the deeds recorded. Even here, however, the bold spirit of modern criticism has flung its doubts on the theme of our schoolboy exercise and delight. We have already adverted to a general suspicion of partiality in the narrative. Other charges have been made impeaching the accuracy even of the military details, which we are surprised to find that Mr. Merivale passes over without notice. Long ago M. Puysegur, a French general, had broached this scepticism as to certain parts of the work. Frederic of Prussia, sceptic and warrior by profession, and fresh from his own bloody campaigns, avowed that he read the Commentaries in later life with altered faith from that of his earlier days. His friend 
Voltaire - if we may thus profane the name of friendship - living close to the scene of the actions recorded in the first book; and Warnery, upon a minute survey of the same localities, started grave scruples as to the operations by which Cæsar sought to stop the egress of the Helvetians from their mountain territory.* Various passages in the other campaigns have been the subject of like criticism, and doubts even stated as to the authorship of the whole work. In these doubts we cannot acquiesce. Without referring to those other writers, Rohan, Guichard, \&c., who have vindicated the military narrative, we find in Cæsar's Commentaries a perfect reflection of the energy and intelligence of the man, and an entire correspondence with the description which Cicero gives of their style:-Nudi sunt et recti et venusti, omni ornatu orationis, tanquam veste, detracto. We cannot, indeed, conceive any other or lesser artist to have thrown off so completely all ornamental colouring from his narrative, and to have preserved such entire unity throughout the whole. And what record or note has there been left to us of such other author? We may admit the recorded c iticism of Asinius Pollio, that many things were written by Cæsar from the report of others long after the events, and still see in these Commentaries the genuine work of Cæsar himself, and one of the most authentic and valuable records of ancient warfare.

But passing over this question of criticism, there is no question as to the fact that, in eight or nine years, with a force never exceeding sixty thousand legionary soldiers,

* The great stumbling-block here is the fortified wall, reported to have been built to bar this passage; 19 miles in length and 16 feet in height, with ditch, and all other appurtenances to such fortification. The length is deemed by Warnery to be refuted by local circumstances. The execution of the work by one legion, in the time indicated, is thought impossible by others. See on this subject an interesting series of papers in the 'United Service Magazine' for 1850 . 
Cæsar subdued the whole of Gaul - a mighty and a terrible work. We do not give ready belief to historical numbers, more especially when fields of battle are concerned; but where the struggle was so fierce, and the conquest so complete and lasting, we are obliged to think it probable that the estimate of more than a million of Gauls perishing in these campaigns is not above the truth. It is one of the many unhappinesses of war in every age, that by its absolute or supposed necessities, it leads to acts of cruelty and bloodshed, even where most alien to the feelings of those who conduct it. We have no reason to charge the character of Cæsar with inhumanity, or that gross indifference to human life which Marius and Sylla displayed throughout. On the contrary, we have many instances on record of his personal humanity and forbearance. But the history of these Gallic wars is undoubtedly one of profuse bloodshed; - the natural result of a struggle between disciplined legions and undisciplined multitudes; - of critical positions in the midst of an enemy's country, necessitas in loco, salus ex victoriâ; - of exasperation of the soldiery; - and of intimidation used as an instrument of success. Our vindication can go no further than this; unless, indeed, we were to find it in a parallel with the wars of Frederic and Napoleon, an argument upon which we have neither room nor disposition to enter.

The two invasions of Britain and the passages of the Rhine are episodes in his history, chiefly remarkable as proofs of the indomitable boldness of Cæsar who thus adventured on new lands while those behind him were yet but half subdued. In the fame however, and the fear which followed these deeds, he found an ample equivalent to the risk incurred. At Rome, as well as in Gaul, his passage to Britain was a step towards empire; while, to a mind thus instructed and enlightened, there must have been a further interest in this new land and people beyond the sea. We possess some 
curious evidence from astronomy to show the time and place of his disembarkation in Britain - evidence which may well excite the wonder of those who know not how physical science triumphs in its proofs, even upon the most obscure historical questions; and how deeply chronology is indebted to eclipses and the recorded places of stars for some of its happiest discoveries. Our scientific readers are well aware of the method which Halley applied to this particular calculation; indicating the beach at Walmer or Deal as the place of landing of the Roman legions, and not Hythe, as others from an expression of Dion Cassius had supposed.* From his castle at Walmer the illustrious Warden of the Cinque Ports looked down upon the spot where Cæsar probably first trod the soil of England:- himself equal to Cæsar in military fame and success;- - superior to the Roman, as to all other commanders, in those loftier virtues of a citizen which have secured to him the lasting gratitude of his country.

We must, however, hurry forward to those remaining events in the life of Julius Cæsar, occupying only a few years; but years of marvellous activity and success; which brought him to the very steps of the throne he was not

* The single statement of a full moon occurring on the fourth night after Cæsar's arrival off the cliffs of Dover gives basis to the calculation. His passage across the Channel was made early in the morning of the 26th of August. That retrospective reckoning, which is one of the prime powers and wonders of astronomy, enabled Halley to determine that there were two full moons in August, 58 B.c. ; and the narrative shows the last of these, at midnight on the 30 th, to be the one recorded. The course of proof then turns to the tides. On the 26th the tide must have begun to flow at Dover at 2 P.M., running northward round the South Foreland. The fleet left its moorings off Dover on this tide, and the length of course Cæsar describes would very exactly suffice, under ordinary circumstances, to bring them to the flat beach of Walmer or Deal. M. Saulcy, in a work just published (1862), also contends for Deal as the landing place. But on the other hand the present Astronomer-Royal - a high authority in all ways - considers that the coast about Pevensey answers better to the indications which the narrative affords us (Archæologia, vol. xxxiv.); while Mr. Lewin, in a recent work, argues with ability that the place of disembarkation must have been in the vicinity of Romney. 
destined himself to fill. The defeat and death of Crassus in his Parthian expedition changed the name of the Triumvirate, but hardly affected the real contest for power, which remained, as before, between the two great military chiefs Pompey in the city, Cæsar in his camp. Jealousies and causes of rupture multiplied as time went on. The death of Julia - more deeply lamented, it would seem, by the husband than the father - broke asunder one bond of union between them. The anarchy in Rome, fomented rather than repressed by Pompey, had placed him in the condition of sole Consul of the Republic; - an anomalous admixture of old institutions with the aggressions and tyranny of the existing time; and certain not to subsist long, when so palpable a fiction in itself, and so entirely opposed to the interests of his matchless rival. On the side of Cæsar, the spoils of Gaul were poured into the city as bribes and largesses. The tribunes were gained to his cause; supporting his claim to a participation in the extraordinary powers thus conceded to Pompey, and to a second consulate, while still holding his province and absent from the city :- demands adverse alike to the letter and spirit of the constitution, and sanctioned only by the breaches already made in it. The claim of Cæsar was refused, as he probably anticipated, under the influence of the senatorial party. He passed the Rubicon, the limit of his province; - boldly, as was his wont; but deliberately, as we are told, and with full knowledge of the importance of an act which has served ever since to describe those steps of bold adventure from which there can be no retreat. His march upon Rome and occupation of the city, while the great strength of his army was yet far distant, were marked by the same dauntless determination. In sixty days from the passage of the fatal streamlet he was master of Italy. The conduct of Pompey in evading the first struggle of arms has been variously explained. Whatever the impulse 
or urgency of the procedure, it was a proof of present disability; disheartening to his adherents and a source of dissension to the party. Though a vast body of senators clung to his flying camp, it was the Senate of Rome no longer, and brought neither counsel nor strength to his cause.

At this time, when the Duumvirate resolved itself into a personal contest between the two leaders, we may believe that the views of Cæsar had become more exactly defined; and that he saw as the needful issue, the mastery of one or the other over the whole fortunes of the Republic. Now, if not before, we may suppose him to have uttered the lines which Cicero tells us were often on his lips, from the Phœnissæ of Euripides, that ' if ever it be fitting to commit wrong, the noblest motive for this is the gain of sovereign power.' Hastening forwards with the tide of events, we find them all still marked with the character of the man, ever more energetic and capable as the difficulties were greater. While Pompey was loitering with his senators and troops in Epirus, Cæsar pushed across the Alps and Pyrenees into Spain;subdued in an arduous and critical campaign of forty days the large legionary army opposed to him on the Ebro;-overcame the remaining Pompeian forces on the Guadalquivir; - and, when hardly yet known' to be on the shores of the Atlantic at Cadiz, suddenly appeared at Marseilles to decide the surrender of that city, long vainly besieged by his subalterns. To estimate rightly what such marches and victories were, it is needful to revert to the aspect of these countries at the time, to the state of the mountain roads, to the dangerous and scanty means of navigation on the seas. When we are told that Cæsar himself often crossed rivers by swimming on inflated skins, overtaking his own couriers in the speed of his course, we can form some idea of the difficulties encountered, and of the personal energy by which they were overcome. 
After quelling by his single presence and speech a mutiny of some of his legions at Placentia, he reappeared at Rome; confirmed his authority there by wise and salutary acts; and then, with such part of his army as he could collect in time, threw himself suddenly upon the coast of Epirus, there to confront for the first time his great adversary. A protracted contest followed near Dyrrachium, of refined strategy and alternate blockade by sea and land. The inferiority of Cæsar's force, even after being joined by fresh legions, exposed him to a severe check, which had the effect of suddenly transferring the war across the mountain barrier of Pindus to the plains of Thessaly. Here the momentous battle of Pharsalia closed the struggle, and decided the fortunes of Cæsar. His hardy legions, like the iron regiments of Cromwell, confident in their commander, won complete victory over the numerous but more courtly and effeminate army opposed to them. The field is to this day unchanged in its main features. The stream of the Enipeus is still seen winding across it; a village occupies the site, and yet bears the name of Pharsalus. We ourselves have twice trodden over this ground, and been able to note, without any great ambiguity, the main localities of a conflict thus famous in history.*

Cæsar permitted no pause in the pursuit of his rival, or in the interest of his own career. With a hardihood which might be censured as rashness, were it not so constantly justified by success, he was still ever in advance of his army.

* Mr. Merivale's account of the battle is somewhat obscure in its topography. His supposition of change in the course of the Enipeus is not necessary to reconcile Cæsar's narrative with the actual localities. The vestiges of the ancient walls of Pharsalus around the modern village, the position of the several hills bordering the Enipeus to the south, and the interval between these hills and the stream, all accord with the events as described to us by Cæsar. On this subject we may best refer to the authority of Colonel Leake. In a memoir published by the Royal Society of Literature, this most learned and accurate scholar satisfactorily illustrates the whole campaign, and very especially the battle of Pharsalia, upon his personal knowledge of the ground. 
Rapidly traversing Thrace and Asia Minor, he embarked for Egypt, where, though he found Pompey slain, no meaner talent or fortitude than his own could have saved him from the peril to which he exposed himself amidst the populace of Alexandria, infuriated by his bold demands and interference with their national affairs. Rescued from this danger, the history of Cæsar for a moment changes its complexion. We find the warrior and statesman yielding himself to the blandishments of Cleopatra, and the companion of the Egyptian queen in the lawless and luxurious revelries of the East. We hardly know in what degree this picture has been coloured by hostile scandal or poetical embellishment. Both, we suspect, have been at work with the story; though we must add that Mr. Merivale gives larger belief to it, and describes the character of Cæsar as permanently changed by the vices and indulgences of his Egyptian life. Thus he says, in a passage with which we cannot readily agree -

But the sorceress of the Nile had not only corrupted the Consul's patriotism; she had enchanted him with the poisoned cup of Canopic luxury. She had taught him to despise as mean and homely the splendour of the Circus and the Capitol; she had imbued him with the gorgeous and selfish principles of Oriental despotism, and debased him to the menial adulation of slaves, parasites, and eunuchs. .... If from henceforward we find his generosity tinged with ostentation, his courage with arrogance, his resolution with harshness; if he becomes restless and fretful and impatient of contradiction; if his conduct is marked with contempt for mankind rather than with indulgence to their weaknesses, it is to this impure source that the melancholy change is to be traced.

We confess that we always view antithetical writing with distrust; nor do we find in the remaining acts and events of Cæsar's life any evidence sufficient to justify the conclusion here denoted. Though by no means exempt from human weakness, we believe him to have been a man who could dally with luxury and flattery without being subdued by 
them. We find him breaking loose from these supposed bonds on the first intimation of a war in Pontus, rushing with his usual rapidity to this distant contest, and ending it with more than his usual speed and success. What our author calls his 'arrogant bulletin' - the famous veni, vidi, vici, of this war - is a story too variously told by the writers of the time to admit of its being brought in evidence against Cæsar. Nor have we proof that he sacrificed any real object of his policy either by this dalliance in Egypt, or by the later visit of the Egyptian queen to Rome. Mark Antony might lose his world for Cleopatra :- Cæsar could not be thus conquered. Yet, while rejecting the probable exaggeration of anecdote and poetry, we cannot willingly part with the whole of the picture handed down to us. A passage of Suetonius places before our imagination a barge on the Nile, bearing the splendid freight of Cæsar and Cleopatra - the majestic Roman who changed the face of the world, noble in person and feature, great in intellectual power as in war - and the royal sorceress of Egypt, bent to win her splendid prize, and by the very witcheries which long after led a conqueror captive to his ruin. But we need for such a scene as this our own Shakspeare; whose gorgeous picture of Cleopatra on the Cydnus is one of the many marvels in his unapproached delineation of this woman;a portraiture ranking as a whole among the truest and most felicitous of all his wonderful works.

Scarcely had the untired Cæsar reached Rome from his victory over the son of Mithridates in Asia, when he set forth again with his army for Africa to encounter the powerful force collected there by Cato and Scipio. The conflict at Thapsus closed the campaign at once; and gave a motive, though we are far from believing a necessity, to the tragic end of Cato. On this subject Mr. Merivale puts before us a striking and eloquent passage. 
From Africa Cæsar returned to Rome, and celebrated there a quadruple triumph of greater magnificence than any that had gone before, but with all the strange and ferocious exhibitions belonging to this festival. It was a needful concession to national usage; - whether made willingly or not, we have no means of affirming. Much discretion was required in the selection of the subjects for triumph; since civil wars had been so closely interwoven with foreign, that his greatest exploits and successes were studiously kept out of sight. And scarcely indeed were these shows ended, when he was again summoned to the field to put down the large insurrectionary army which the sons of Pompey had assembled in Spain. In twenty-seven days - celeri festinatione, as his historian well says - he was with his forces in Andalusia. The bloody but decisive day of Munda, where 30,000 soldiers were left on the field, and the victor himself exposed to imminent personal danger, closed this last formidable antagonism and the military life of Cæsar. It is a point of time when even those who most deprecate war in all its forms may look back with astonishment, if not with admiration, at the wonderful career of victory so terminated. Whether we consider the vast countries and distances thus traversed in Europe, Africa, and Asia, - the battles gained, - or the conquests effected, we cannot but feel that Cicero has well applied the term of $\tau$ epas to express the activity, the vigilance, the sagacious daring of Julius Cæsar. He is indeed a prodigy in the history of manknd.

After his final campaign he returned to Italy: this also was for the last time. Though absent for many months, the awe inspired by his name had protected the city against all turbulance or innovation; and he now came back, single and supreme, the arbiter of the future destiny of Rome and the known world. Already three times declared Dictator, he was now named such for life. The consulship was given to 
him for ten successive years; a crown of laurel and triumphal robes were allotted to his public appearances; his head, for the first time, was stamped on the public coinage. All these things were outrages on old custom and feeling: - they betokened the greatness of the change not less than of the man who had brought it about. The title of Imperator given at the same time, had much less import and weight then, than it has since obtained. Though never before prefixed to a name, it avoided the odium which was still attached to the style of King. But associated as it was in Cæsar with more than regal power, it became the badge of high sovereignty, and descended through a long line of Roman Emperors (or those so called) to the times in which we now live.

This last epoch of Cæsar's life, at which we arrive, was of little more than eight months' duration. It was occupied in various useful reforms and legislation; the sequel in principle to the measures which at prior times he had proposed, or partially carried into effect. He indulged the people with the sports and shows which usage and policy required; but his aims were evidently beyond these things; and from what he actually did, we have reason to believe that though his destined term of life was nearly completed, his designs were far from being so. We have no exact knowledge of the date of his several measures; but as far as we can see, they had for their basis the establishment of order in the City and provinces, the suppression of existing abuses, and the change or extinction of those old institutions which were now effective only in lending a shelter to them. His liberal extension of the rights of Roman citizenship was but a sequel to the policy of his whole life on that point; and the result doubtless of his conviction that what was not yielded peaceably would be, sooner or later, extorted by violence. The large increase of the Senate, and the admission of numerous 
foreigners into this body, while it seemed to repair the breaches made by the civil wars, and flattered the new citizens from the provinces, was virtually an annihilation of this part of the old Roman government, already debased by luxury and intrigue, and incapable of fulfilling its ancient functions. With this same view probably, he shortened the term of the Consulate; an office which was now sought for by turbulence and bribery, and exercised only for party purposes. The consular and prætorian provincial governments were also abridged in duration; for the wise purpose of checking the gross speculations and abuses which had grown up in this part of the Roman administration. Larger admission was given to all public offices, with less limitation as to rank and age; a measure which tended to destroy the influence of those great families (prceclaro nomine tantum insignes) who made their ancestral fame an avenue to public functions, which they dishonoured by their acts. As Censor, Cæsar enacted certain sumptuary laws which, had he lived, he would doubtless have enforced; and began various improvements in the judicial system, and especially in criminal law. $\mathrm{He}$ established colonies of veteran soldiers on a plan which procured exemption from many of the disorders consequent on long civil war. He appointed a commission, and furnished a scheme for a land-survey and map of the whole Roman dominion; and with the same zeal for practical good and knowledge of the resources of science, he accomplished that reform of the Calendar which alone would have preserved his name to posterity.

While thus indicating the general principles upon which Cæsar guided his government, there remains the curious enquiry - What would have been his own future course and position in it, had his life been prolonged? Master of the Roman world he was:-Master he must have continued, under one title or other. No conspiracy by open arms could have suc- 
ceeded, or been attempted, in the face of his military renown; and the resignation of Sylla, of which he is said to have spoken disdainfully, could never have seemed to him other than a warning; - since it had given fresh scope to those civil disorders which he, above all men, knew the necessity of bringing to an end. His personal ambition doubtless here concurred with, and strengthened these convictions of his reason. But power, even the most entire, cannot well subsist without some external form or title; and the turba Remi resembled the populace of every age and country. We know not how far the story of the kingly crown being offered to him, and of his reluctant refusal of it, is worthy of reliance; but we suspect that the officiousness of friends, or the malignity of enemies, were more concerned in this matter than the will of Cæsar himself. There never was a man less governed by mere phrases, or who would more readily abandon an outward show for the reality that was before him. The new prefix of Imperator sufficed for the designation of that power, which, in default of direct issue, he would probably have conveyed downwards to the very successor on whom future events actually conferred it. We further believe, on all the evidence of his acts and character, that his own rule would have been one of vigour, tempered by moderation and humanity, - of firmness to repress sedition, - and of wisdom to organise new institutions where the old ones had become impotent for good.

Two anecdotes, unconnected with politics, belong to this last period of Cæsar's life, which have the greater interest from the time of their occurrence. One is the narrative, contained in a letter from Cicero to Atticus, of the visit paid by the great master of Rome to its great orator, at his villa near Puteoli. The details of the interview, and the dinner given to the Dictator and his numerous attendants, illustrate most agreeably the manners of the day; but far more 
strikingly describe the two remarkable men thus brought together for the last time; - both deeply concerned in the public events of the preceding twenty years, - - both destined to perish by a violent death. We would willingly invite the attention of those of our readers who may have forgotten it, to this curious and characteristic letter. We have always been especially entertained by the frank confession of Cicero to Atticus, that Cæsar was not a guest to whom he could say ' Pray, pay me another visit on your return'-(semel satis est); and also by his acknowledgement that no serious matter

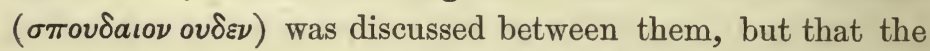
conversation turned mainly on literary topics. The motive for this restraint may readily be found in the history of the time, and in the relative position of Cicero and his great visitor: - the direction of the conversation, we doubt not, rested entirely with the latter. Even with such restraint upon it, who does not wish that a Boswell had been present to record what passed ?* The other anecdote to which we allude, has less certain authority for its truth but great internal probability. Cæsar was the guest of M. Lepidus at supper at Rome. After the repast, a conversation arising on the question which was the most desirable manner of death, the Dictator pronounced as his opinion that it was 'the one most sudden and unexpected;' - a sentiment natural to the man, and which, in a qualified form, was afterwards repeated by his first successor in the empire.

On the very day, as we are told, after this supper, Cæsar's life was ended by that murder which seemed at the time fated to change the condition of the world. It would be difficult indeed to name any single incident of deeper interest.

* We gather further from this letter, that Cæsar had other and more ordinary powers of making himself an agreeable guest at dinner. 'Edit et bibit $a \delta \epsilon \hat{\omega} s$ et jucundè; - opiparè sané, et apparatè.' We are surprised that Mr. Merivale should so slightly press this curious document of Roman life. 
On the one side we have the character and dignity of Cæsar, the power he had acquired, and the prospect of this power being permanent as a new form of Roman rule:- on the other side, the publicity of the assassination, and the condition and qualities of the men whose swords were thus

made rich

With the most noble blood of all this world.

The death of Cæsar, like that of Cato, has almost lost its historical character in the poetry and romance of later times, which have appropriated to themselves an event thus signal in all its circumstances. Shakspeare probably comes nearest, as he generally does by his almost superhuman instinct, to the reality of the case. His Cassius, too, is at once a faithful transcript from history, and a picture of the jealous and ambitious conspirator of all ages. The Brutus of his play was necessary to the dramatic effect which he so well understood; and accordingly we find that, while closely adhering to historical truth in most parts, he has pitched the character of the Roman patriot somewhat higher than the level assigned by contemporary authorities. History has been defined 'philosophy teaching by examples;' but we have little faith in the force of such examples, as opposed to living and current events; and, removing all artificial colouring from the act before us, we are disposed to regard it as the result of jealousy, ambition, and other personal feelings, much more than of the pure love of liberty or the desire of restoring the grandeur of Rome. The assassination of Cæsar inflicted another civil war on the State, without really retarding the great change which was on the eve of accomplishment. Had his life been prolonged, it is probable that his enlightened vigour of administration would have given a better basis to the empire, and a higher model and precept to his successors, than the more subtle and temporising reign of Augustus was able afterwards to afford. 
This act of blood closes the narrative now before us; our author's final chapter being occupied by a delineation of the character of Julius, and of the condition of Rome at the time of his death. The former subject has been variously handled, according to the complexion of the writer's mind, or that desire of framing a theory of motives and events which is so often observable in those who undertake to record them. We have no desire to shelter moral failings, or to palliate the evils arising from ambition and war; yet we must express our belief that Dr. Arnold has coloured his Cæsar too darkly. Mr. Merivale is less austere; and a passage at the beginning of the third chapter, shows his comprehension of those high qualities which designate to all posterity this greatest of Romans. We have, however, to complain that his portraiture is somewhat broken and unconnected, giving the feeling of a certain want of congruity; and occasionally it would seem that even a line of Lucan, or an anecdote of Suetonius, has sufficed to produce a change of opinion. In one place, for instance, he speaks of the ' perfect simplicity of Cæsar's character ;' in another, of 'his gratuitous indulgence to his passion for personal display;' qualities hardly compatible in themselves, even with every allowance for those disparities of which we have elsewhere spoken. That the error lies in the latter passage we entertain no doubt. The mind of Cæsar had, we believe, very much more of singleness and consistency than is often met with in history; but the events and conditions of his career were so various and extraordinary, that it would require much critical research and discrimination to present a picture of him which might satisfy all the requirements of truth. On the whole we may say, without attempting such an analysis, that while his public course during the last sixteen years of his life was one of almost constant and bloody warfare, his natural temperament seems to have been hu- 
mane, and free from that remorseless cruelty which stained the career of so many of the public men of his age. Whatever of moral contradiction there may seem in this, experience teaches that the conditions are compatible; and in the instance before us we have proof sufficient to justify the opinion given. The 'gaudensque viam fecisse ruinâ' of Lucan is the phrase of the poet, and not the truth of the historian. Amidst the thousand private animosities which civil wars engender and envenom, we do not call to mind a single action of Cæsar prompted by private malice or revenge; - many of humanity and generosity to enemies who fell within his power. Whether he was a man of warm affections may admit of more doubt. We incline to think not; though without any certain evidence by which to decide the question. He had several friends indeed-Oppius, Calvena, Balbus, \&c. - who were deeply attached to him; and various acts of his personal kindness to them and others are on record: but his superiority to all around him was such, that it is difficult to measure the feelings in this case by any ordinary rule. All the strongest traits of Cæsar's mind were intellectual; and we doubt whether softer sentiments, passion, or romance, had ever any very strong hold upon him. A tragedy, indeed, was among the number of his literary works; but it no longer exists, nor have we any such accounts of it as to affect our guesses.

The profligacy of his early course - partially, as it seems, carried on into his later years - has been a main allegation against him in all succeeding times. We cannot accuse him of intemperance at table, since Cato remarks that he was 'the only one who went forth sober to the overturning of the commonwealth.' But the charges of other sensuality admit no excuse or palliation; unless we find such in the general corruption of the age, or in some suspicion that the public eminence of Cæsar might have led to exaggerated 
statements of all that regarded his demeanour in private life. Making every allowance for such over-colouring, however, we are still unable to dismiss the general imputation. Temperament, temptation, opportunity, were all on one side, without a single aid from religion or moral discipline on the other. Two or three small incidents are presented to us as proofs of superstitious feeling; but we believe them to have depended rather on a politic or careless conformity to popular sentiment; for Cæsar lived, as Virgil did,

Al tempo degli Dei falsi e bugiardi;

and it was impossible that an acute intellect like his should have submitted itself for a moment to the puerile absurdities of the Grecian or Roman belief, or derived motives to virtue from sources thus scanty and impure. He lived without religion, on the very verge of that time which brought new light and truth into the world.

As to the intellectual qualities of Cæsar, it is needless to say more. They are inscribed on every page of his life and history, and are the subject of constant admiration to his contemporaries as well as to succeeding writers. A single sentence of Cicero, than whom no man was better entitled to judge, is a relation to all future time of that combination of faculties which has rarely, if ever, had its parallel: 'Fuit in illo ingenium, ratio, memoria, litterce, cura, cogitatio, diligentia.' Pliny, in his Natural History, recording the most noted examples of intellectual power, instances Cæsar as possessed of an innate vigour of mind transcending all others; able without confusion to embrace various subjects at once, to dictate clearly on each, and to pass .with the celerity of lightning from one to another. Omitting the many other testimonies of the same age, we may take the eulogy by Drumann as a brief and just statement of what was achieved in various ways by this wonderful force and capacity of mind. 
' $\mathrm{He}$ was great in everything he essayed: as a captain, a statesman, a lawgiver, a jurist, an orator, a poet, a grammarian, a mathematician, and an architect.' We have only to object to this eulogium that it assumes a parity of excellence in points where we must suppose that there was much real inequality. But nothing is stated here which Cæsar did not actually accomplish; and his mind rose so high above mediocrity, that, even where our evidence is imperfect, we can believe some part of his genius to have been conveyed to all he undertook.

With the exception (and this a doubtful one) of Frederick of Prussia, Cæsar is perhaps the only great commander who adds the fame of literature to that of war. Near the close of his career he established a Greek and I atin library at Rome, selecting Varro as his librarian. Unhappily all his writings are lost to us except the Commentaries; a fact which, regarding the author both in his own greatness and as the head of a long line of sovereigns, may reasonably excite surprise as well as regret. We are indeed imperfectly informed as to the mode in which the manuscripts, forming the literature of those days, passed into circulation and were transferred from one generation to another; but still it must appear strange that so large a part of the writings of a man like Cæsar should have disappeared from the world. It is related that he composed his grammatical treatise, De Analogiâ, while travelling through the Alps; and a poem called Iter during a journey in Spain. The former was dedicated to Cicero; and from a fragment of it still preserved, we find that Cæsar first proposed the name of ablative for a case not known to the Greek Grammar. Looking at other points in his character, we are half inclined to believe that he wrote them solely for his amusement while on the road; and that, indifferent to literary fame, he took little care to multiply the copies which might secure transmission to later times. 
Of his other writings we most covet the satire of the AntiCato, the treatise on Auguries and Presages, and his tragedy of Edipus. We venture no opinion as to the probable merit of these works, beyond the general inference already stated; but may hazard a conjecture that the poetical and imaginative part would have added least to his reputation. Niebuhr has somewhere remarked that there is no witty saying of Cæsar on record. It is difficult enough to define wit in any form, and we should hardly go to a German professor for aid in the definition; but if pithy and pointed expressions and retorts come under the term, we know that Cæsar had such at command. Some specimens live indeed in every scholar's memory; and if his collection of apophthegms - the mucrones verborum, as Lord Bacon calls them-had reached us, we should probably have had abundant evidence of his keen apprehension of those great sayings, which form the true wit of every time and language of man.

We are surprised that Mr. Merivale takes such slight notice of the oratorical fame of Cæsar, seeing the strong impression it made upon the best judges of his own age and country. Where Cicero and Quintilian have testified their admiration in terms so remarkable, it is hardly enough to despatch the subject in a short sentence, without reference to these eminent authorities. From one passage in the De Claris Oratoribus, it may be seen how high a value Cicero attached to the opinion of Cæsar regarding his own oratorical powers. What other commander or conqueror, how few statesmen even, have obtained or merited tributes like these, and given by such judges!* Nor can we fail to notice the portrait they convey to us of the noble aspect, attitudes, and voice of

* 'Cæsar autem, rationem adhibens, consuetudinem vitiosam et corruptam purâ et incorruptâ consuetudine emendat. Itaque cum ad hanc elegantiam verborum Latinorum adjungit illa oratoria ornamenta dicendi, tum videtur 
Cæsar, when addressing an assembly. We are able in great part to complete the picture of his outward lineaments from busts, coins, and actual description; all which indicate, as far as mere outline can ever do so, the high intellect and masculine vigour of mind belonging to this wonderful character.*

We have, we hope, shown that we are disposed to augur well of Mr. Merivale's large and bold undertaking. We shall look with interest to his next volumes, as the history of that subtle Sovereign who, under the specious show of old names and forms, succeeded in giving to a disputed and divided power all the unity and integrity of an ancient monarchy. The character of Augustus, in his successive capacities of Triumvir and Emperor, has ever been counted an historical problem; and with every allowance for the frailty and changes of our nature, it still is not easy to reconcile all the incongruities which the acts of his life bring before us. Beyond this period, our author will acquire the aid of Tacitus to his labours; - an authority and a guide not less admirable than is Thucydides to the historian of Greece; both possessing qualities which may well serve as instruction to those writing history in all time to come. Without

tanquam tabulas bené pictas collocare in bono lumine. **** Splendidam quendam, minimèque veteratoriam rationem dicendi tenet, voce, motû, formâ etiam magnificâ et generosâ quodammodo.'-Cicero de Claris Oratoribus.

'Quem huic antepones eorum, qui nihil aliud egerunt? quis sententiis aut acutior aut crebrior? quis verbis aut ornatior aut elegantior?'-Ibid.

- Caius verò Cæsar si foro tantum vacasset, non alius ex nostris contra Ciceronem nominaretur; tanta in eo vis est, id acumen, ea concitatio, ut illum eodem animo dixisse, quo bellavit, appareat.'-Quintilian, lib. 10.

It is obvious that Quintilian would not thus have expressed himself, unless some at least of Cæsar's speeches had been extant in his time.

* It is satisfactory to me to find in the great work of Mommsen on Roman History, recently published (Römische Geschichte, 1857), an entire concurrence of opinion in all that relates to Cæsar's character and career; and a like agreement in the estimate of his great rivals and contemporaries. 
exacting from our author the rigorous brevity of Tacitus, which would be rendered impossible by a regard to modern taste, as well as to the larger field of critical history over which his course lies, we still think that something may be gained from this great model; whom it is impossible to study without acquiring vigour from his style; or without sympathising in that high spirit of philosophy, and utter disdain of all that is false and frivolous in the world, which marks everything he has written. 


\section{PHYSICAL GEOGRAPHY OF THE SEA-THE ATLANTIC OCEAN.*}

[EDinburgh Review, Aprix, 1857.] $\mathrm{N}$ the earlier days of this Review the teaching of geo-
graphy, as then understood and practised amongst us, was a dry and barren task; tedious to the teacher, distasteful and of slender profit to the scholar. Bald catalogues of easily forgotten names (locorum nuda nomina, as Pliny calls them), uninformed by science and scantily illustrated by history, formed the staple of the study. Nor was any part of education more defaced by the coarser mechanism of bookmaking. Errors of fact, and even of nomenclature, were perpetuated from one edition or compilation to another; with little regard to original accuracy, or to the changes going on in the world. And even where some fragment of history or physical science broke in upon the network of names, it was often of doubtful authenticity, or too partial and detached to give real knowledge or gain hold on the memory. This is not an exaggerated view of the manner in which geography was generally taught in England down to a recent period.

The more exact study of history had already improved the

* 1. The Physical Geography of the Sea. By Lieut. Maury, U.S. Navy. London and New York, 1856.

2. Considérations Générales sur l'Océan Atlantique. Par Philippe de Kerhallet. Paris, 1853. 
methods, and extended the sphere of geography, before physical science had fairly annexed itself to the subject; creating new associations of high interest in themselves, and fertile in their influence on the condition and welfare of mankind. Humboldt is the philosopher who has especially contributed to the establishment of physical geography as a branch of science. The natural phenomena, indeed, upon which it is founded, being ever present and patent to observation, could not have escaped record; and this record was becoming continually more copious through its connection with other branches of natural knowledge. But there was yet wanting a clear specification of the scope and objects of the science thus gradually evolving itself; and of the methods best fitted for their attainment. It is this which we owe to Humboldt's peculiar genius, aided by the vast resources derived from travel and personal observation. The globe was to him much more than a mere superficial delineation of land and sea, of mountains and rivers, of terrestrial divisions and other human landmarks. His researches comprised, under a closer and more connected view, those great physical characters of the earth's surface through which alone we can learn the changes it has undergone or is yet undergoing; - the physical elements and forces which have been coneerned, or are still active in producing these changes; and the agents and means by which change is limited and general stability maintained.

In assigning to Humboldt the foremost place among those who have given to Physical Geography the name and character of a science, we must add that this great field has since been full of labourers, zealous in their work, and bringing to it numerous aids and appliances furnished by other branches of natural knowledge. Scarcely in truth is there one which has not been made to contribute, directly or indirectly, to Physical Geography in the full meaning of the term. When 
earth, ocean, and atmosphere all come within its sphere, as well as those great and mysterious forces - gravitation, heat, light, and electricity - by which these several portions of our planet are so powerfully and incessantly acted upon, it will be seen how closely the subject is linked with every other research into the world of nature around us. Our countrywoman, Mrs. Somerville, has well expounded these relations in her admirable volumes on Physical Geography. The Physical Atlas of Berghaus, a valuable German work, preceded the publication in this country of the more extensive and elaborate 'Physical Atlas of Natural Phenomena' by Mr. A. Keith Johnston, of which it would be difficult to speak in terms above the mark of its actual merits. Embracing every part of the subject, it delineates to the eye as well as the mind - and far better than by any verbal description - those complex relations of physical phenomena on the globe, which are the true foundation of Physical Geography.

Of all branches of science, no one contributes so largely to our knowledge of the actual condition of the globe as Geology. By drawing its conclusions from a long series of ages, this science has given us the means, in no other way equally attainable, of studying and explaining the present aspects of the earth we inhabit. Such are, the power obtained through the study of fossil remains, of identifying strata in localities the most remote, and thus fixing the common epoch (however long its duration) of certain states or changes of the crust of the globe; - the facts discovered, which prove the gradual upheaval of portions of the earth's surface and the slow depression of others; - the proofs from the inclination and contortions of strata, from the alterations of the older strata, and from the position and elevation of the unstratified rocks, that other changes, more abrupt and violent, have occurred from subterranean forces of expansion or 
contraction; - the evidence derived from the direction, parallelism, and other aspects of mountain chains, as to periods of contemporaneous elevation; - the influences upon climate of lands elevated above the sea or depressed below it; - and further, the whole history of that coral creation, by which, under the slow working of microscopic forms of animal life, islands and reefs are raised from the depth of the ocean, to become the habitation of other and higher existences.

We have thus far spoken of Physical Geography in its largest acceptation. It has, however, of late been submitted to a process of division, made needful by the multiplicity of the objects it includes; and the phrase of ' Physical Geography of the Sea,' proposed by Humboldt, is the title of the first of the works now before us. Under this title its author, Captain Maury, of the United States navy, includes all that concerns the great domain of waters over the globe; - the oceans, seas, and basin lakes into which they are distributed; - their various depth, temperature, and saltness; - the currents which permanently or periodically pervade them; - the phenomena of the tides; - the phenomena of winds, whether constant or irregular, whether the steady trade breeze, or the hurricane and cyclone; - the law of evaporation belonging to different latitudes of the watery world; - the less known, yet certain agency of magnetic or electrical forces; - and the mutual influence of ocean and land in all these physical actions and changes. This summary statement shows how vast and various are the objects in the division of science thus proposed. We find further reason for its adoption in the importance of all these objects to the principles and practice of navigation; - a matter of supreme weight in these days, when the ocean in its every part is covered with ships; shaped in new forms, moved by new forces, destined to new shores, and seeking to attain by new routes the highest speed of transit. Facts and phenomena, before unobserved or bar- 
ren of result, are now eagerly appropriated; and, by the science and ingenuity of man, made to minister to the purposes of human intercourse over the globe. The ocean, once an obstacle, has become the high road of nations. If steam has worked its wonders on the land, so it has also on the sea; and under a form surpassing, in grandeur of force and effect, all the other operations of this great agent of human power. Iron, that material which ministers in such endless ways to the uses of man, has scarcely less efficiency on the ocean than on land; and we have at this moment in progress before our eyes, gigantic applications of it to the building and propulsion of vessels, both of war and commerce, such as the world has never before seen.

Acquiescing fully, then, in the name and distinction of 'Physical Geography of the Sea,' we may add that we consider Captain Maury a worthy interpreter of the great phenomena included under this title. Attached as Superintendent to the National Observatory at Washington, he has used this honourable position, with much zeal and intelligence, in forwarding objects of singular importance to his own country and to ours, and of general interest to all nations of the world. He published some years ago his 'Wind and Current Charts,' a valuable precursor of the present volume. To his assiduity we owe that conference held at Brussels in August 1853, in which were found representatives from England, France, the United States, Russia, Sweden, Denmark, Holland, Belgium, and Portugal;-occupied, at the very time when war sternly impended over Europe, in organising plans for those cooperative labours on the ocean; those methodical records of winds, currents, tides, and temperature, which provide for the peaceful interests and progress of commercial navigation over the globe. Austria, Prussia, the Hanse Towns, Spain, and Brazil, subsequently offered their cooperation in the same great scheme. With observa- 
tions thus multiplied on every side - the log even of the common merchant brig being admitted to its share in the work - facts will speedily become numerous enough to yield results of the highest certainty and value. The method of averages, now so potent an aid to research, has especial application here, furnishing a secure approach to conclusions which no detached observations could reach.

Though Captain Maury claims all seas for his province, the larger portion of his volume is occupied with the great Ocean which separates the Old from the New World; a very natural effect of the supreme importance of the Atlantic to the commerce of nations, and of the greater knowledge thus attained of all its physical phenomena.* Considered as a scientific treatise, our author has not done full justice to himself, or to his subject, by his manner of dealing with it. We are unwilling to be hypercritical where there is so much real merit; but it is impossible not to see in his work a desultory desire for novelty, frequently going far beyond the bounds of true science, and venting itself in a phraseology which loses its force and effect by being too sedulous to attain them. With a little more constraint upon his speculations, and a clearer separation of fact and hypothesis, he would be a valuable scientific writer. With somewhat less intention of fine writing, he would be an eloquent one.

It is with reluctance that we advert to another characteristic of this volume:- we mean the very frequent and incautious reference to passages in Scripture; not solely for illustration, but even as authority for physical truths, or argument for hypotheses still unproved. Lieut. Maury is evidently a man of strong and sincere religious feelings, and

* It will be seen that we have given place on our list to another work, by Captain Philippe de Kerhallet of the French Navy, having more especial relation to this Ocean; less scientific in its character than that of Captain Maury, and less animated and vigorous in its descriptive part, but nevertheless containing much that is of great practical value for navigation. 
we honour the earnestness with which he expresses them. But he unhappily does not see that in forcing Scripture to the interpretation of physical facts, he is mistaking the whole purport of the sacred Books. In thus appropriating their language, he annuls its bearing on matters of deep concern by applying it to objects and cases of totally different nature. This pia deflexio, as it has been termed in instances of more serious import, must ever be regarded as an injury done to real religion; and we are anxious now, as at all times, to enter our remonstrance against it.

The passages thus misapplied are chiefly taken from the Old Testament - the Psalms, the Book of Joo, \&c., - which, in the pictures they give of the works and wonders of creation, need borrow nothing of that science they do not profess, to render them to all ages the most sublime eulogies of the power and wisdom of the Creator. One example only we will cite, to show how much of error may enter into this loose method of dealing with scriptural authority. After a passage, too laboriously ornate in its diction, where our author speaks of the allusions in the Bible to the laws of nature as involving, under figurative language, hidden meanings which are only disclosed by the later revelations of scieuce, he quotes among other instances the striking text from Job (xxxviii. 31), "Canst thou bind the sweet influence of the Pleiades?' or, as he gives it, 'Canst thou tell the sweet influence of the Pleiades?' And this sublime but obscure interrogation he considers as solved by the recent views of Professor Mädler of Dorpat, which represent the star of Alcyone in the Pleiades as the centre of gravity of that vast sidereal system, to which our globe belongs as a small and subordinate planet.

Here we must remark, but without wonder or reproach, that he is ignorant of the controversy as to this text, which has engaged the learning of Gesenius, Rosenmüller, Mason- 
Good, Herder, and other scholars; leaving the interpretation still very ambiguous. He seems himself to have quoted from some translation which doubtfully takes half the sense from

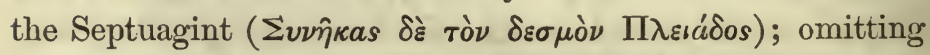
altogether the conception of a link or binding together, which is kept in our authorised translation, and which so happily applies to the close and beautiful aggregation of stars in this group; - an aggregation of such kind that astronomers have calculated the chances to be more than half a million to one, that they could not have been thus set in the heavens by accident alone.

The latter part of the passage in question is also of doubtful interpretation; and we may well ask therefore, whether this is a text upon which to establish or confirm a physical fact? But, further, our author assumes in his argument that Mädler's conception of the Pleiades, as the centre of the sidereal 'system, is ' all but proved;' forgetting or ignorant that few astronomers have recognised it as more than a magnificent problem awaiting solution from future research. Sir J. Herschel especially has given a distinct reason for distrusting this opinion, in the distance of the Pleiades from the plane of the Milky Way; which plane must presumably coincide with and define that of any general movement of rotation in the stellar system, should such exist. The science therefore of this comment is as doubtful as the scriptural quotation to which it is appended.

It may seem that we have dwelt too long on this matter; but we must repeat in justification our earnest desire that the authority of Scripture should not thus rashly be pledged to facts and opinions with which it has no concern, save in so far as it describes the visible manifestations of creative wisdom, beauty, and power. The example just given we consider to be an apt illustration of the errors usually committed in this method of argument. Though less frequent 
than formerly, we still find them in some controversies of recent date; gaining a prompt influence over the public mind; as injurious, we believe, to the interests of religion as of the sciences thus forced into contact with it.

We come now, and with more satisfaction, to the legitimate object of Captain Maury's work, - the great watery empire of the globe; the aspects and phenomena of oceans and seas; their various physical relations, as well to the continents and islands they encircle, as to the atmosphere incumbent over all; and that farther relation they bear to the efforts of human industry, intrepidity, and skill, which have rendered the most distant paths of ocean open and assured to all nations of the earth. The Atlantic is the especial object of our author's labours; and accordingly we find the first parts of his volume occupied almost exclusively with this ocean. Though we may explain the preference, we. cannot wholly acquiesce in it as preliminary to a physical history of the sea at large. The subject requires to be prefaced by those more general views of the distribution and relative configuration of water and land over the globe, which form the very foundation of physical geography, and are fertile in curious and important conclusions. Facts which, if stated at all, are loosely and incongruously scattered over the volume, ought to have been put before the reader in some connected form; as indicating the nature and magnitude of the objects concerned. Captain Maury plunges him at once into mid-ocean, without compass or guidance over its world of waters. A greater familiarity with the writings of Humboldt, Ritter, Von Buch, and other authors, principally German, who have done so much for the study of physical geography, would have furnished both model and materials for a preliminary chapter, such as we desire for a work bearing this title and dealing with objects so vast and various in kind.

We may cursorily state here, in illustration, a few of those 
general facts, to which our author might fitly have given the priority suggested. First, the proportion of sea to land, determined as being nearly three to one;-or in other words, three-fourths of the surface of the globe is actually covered with water. Next, the fact (important in suggesting a disparity in the physical forces which have acted on the two hemispheres) of the great excess of land in the northern hemisphere over that of the southern, being in the ratio of 11 to 4 ; - on which condition depend the curious results that only 1-27th part of existing land has land diametrically opposite to it in the other hemisphere, and that the line of the equator, as it girdles the earth, rests on the ocean for five sixths of its length. Another mode of estimating the proportions and local relations of land and sea is obtained by halving the globe longitudinally on the meridian of the Canaries; when a much larger proportion of sea will be found on the western half or hemisphere so defined, than on the eastern. The main fact of the great predominance of water on the surface of the globe being thus proved, and its mean depth, as we shall see hereafter, approximately determined, we reach other conclusions of high interest to every department of physical science. We shall notice only one of these, in which geological theory, both past and prospective, is more especially concerned. The mean elevation above the sea level of all the land on the globe - islands as well as continents, mountains as well as plains - is estimated by Humboldt at somewhat less than 1,000 feet. The mean depth of the great oceans of our planet is calculated by Laplace, from the tides and other phenomena, to be at least 21,000 feet. Hence, allowing full margin for errors, the entire submergence of the land might take place, leaving the solid mass of the earth everywhere deeply covered with waters - an elliptical globe of ocean, moving still under the influence of the same sublime laws which had before guided its path through surrounding space. 
This is enough to show what we should have desired as a foreground to the topics of Captain Maury's work. There is undoubtedly much to justify his partiality for the Atlantic as a subject for illustration; and we shall follow his example by limiting our remarks still more exclusively to what concerns this great Ocean - a volume itself in the 'physical geography of the sea.' Indeed, our author devotes his first two chapters to a single current of the Atlantic; but this current, under the name of the Gulf-stream, includes physical conditions so remarkable that we cannot blame the priority thus given to its history. To use his own words :-

There is a river in the ocean. In the severest droughts it never fails, and in the mightiest floods it never overflows. Its banks and its bottom are of cold water, while its current is of warm. The Gulf of Mexico is its fountain, and its mouth is in the Arctic Seas. It is the Gulf-stream. There is in the world no other such majestic flow of waters. Its current is more rapid than the Mississippi or the Amazon, and its volume more than a thousand times greater. Its waters, as far out from the Gulf as the Carolina coasts, are of an indigo blue. They are so distinctly marked, that this line of junction with the common sea-water may be traced by the eye. Often onehalf of the vessel may be perceived floating in Gulf-stream water, while the other half is in common water of the sea; so sharp is the line and such the want of affinity between these waters; and such, too, the reluctance, so to speak, on the part of those of the Gulfstream to mingle with the common water of the sea.

This passage delineates, in terms happily chosen, some of the most striking features of this wonderful stream. But there are yet others to be noted; and we shall dwell somewhat in detail on a natural phenomenon thus remarkable:one, moreover, in which we, the people of the British Isles, have a direct and momentous interest, as well in reference to commerce and navigation, as to its certain and various influences on the climate under which we live.

The general description of the Gulf-stream, apart from any present question as to. its sources, is that of a vast and rapid 
ocean-current, issuing from the basin of the Mexican Gulf and Caribbean Sea; doubling the southern cape of Florida; pressing forwards to the north-east in a line almost parallel to the American coast; touching on the southern borders of the Grand Banks of Newfoundland, and at some seasons partially passing over them; - thence, with increasing width and diffusion, traversing the whole breadth of the Atlantic, with a central direction towards the British Isles; and finally losing itself by still wider diffusion, in the Bay of Biscay, on our own shores, and upon the long line of the Norwegian coasts. Its identity in physical characters is preserved throughout the many thousand miles of its continuous flow! - the only change undergone is that of degree. As its waters gradually commingle with those of the surrounding sea, their deep blue tint declines, their high temperature diminishes, the speed with which they press forwards abates. But taking the stream in its total course, it well warrants the vivid description of our author, and the name he bestows upon it of 'a river in the ocean.' This epithet (bringing to

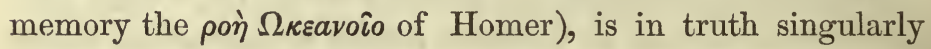
appropriate to this vast current, so constant and continuous in its course, and so strangely detached from the great mass of ocean waters; which, while seemingly cleft asunder to give path to its first impulse, are yet ever pressing upon it, gradually impairing its force and destroying its individuality.

The maximum of velocity, where the stream quits the narrow channel of Bemini which compresses its egress from the gulf, is about 4 miles an hour. Off Cape Hatteras in North Carolina, where it has gained a breadth of 75 miles, the velocity is reduced to 3 miles. On the parallel of the Newfoundland Banks it is further reduced to $1 \frac{1}{2}$ miles an hour, and this gradual abatement of force is continued across the Atlantic. The temperature of the current undergoes similar change. The highest observed is about $85^{\circ} \mathrm{Fahr}$. 
Between Cape Hatteras and Newfoundland, though lessened in amount, the warmth of the stream in winter is still $25^{\circ}$ or $30^{\circ}$ above that of the ocean through which it flows. Nor is this heat wholly lost when it reaches and is spread over the coasts of Northern Europe. The waters, thus constantly flowing to us from the tropical regions, bring warmth as well as abundant moisture to our own islands; and Ireland especially, upon which they more directly impinge, doubtless derives much of its peculiarity of climate - its moisture, verdure, and abundant vegetation - from this source. Were it needful to seek proof of the permanence of the great natural phenomenon of which we are speaking, we might find it in those curious passages of ancient geographers (Pomponius Mela, and J. Solinus Polyhistor, for example) - which describe the peculiarities of the Irish soil and climate eighteen centuries ago, almost as we should depict them now. But the influence of the Gulf-stream does not stop even here. The climate it may be said to convey is diffused, more or less, over the whole Norwegian coast; the aspects and produce of which singularly contrast with those of the corresponding latitudes in North America, Greenland, and Siberia. Other causes doubtless contribute to this effect; but none, we apprehend, so largely or unceasingly.

The influence of the temperature of the Gulf-stream upon animal life in the ocean is very curious. The whale so sedulously shuns its warm waters, as almost to indicate their track by its absence; while yet abundantly found on each side of it. The physical reasons are doubtless the same which prevent this great marine mammal from ever crossing the Equator from one hemisphere to the other - a fact now well ascertained. The various species of fish, which are firm and of excellent flavour in the colder belt of sea upon the American coast, lose all their good qualities when taken out of the Gulf-stream, running closely parallel to it. On the 
other hand, the more delicate marine productions, whether animal or vegetable, which multiply and prosper by warmth, are redundant in the Gulf-stream even after it has quitted the tropical regions whence its heat is derived. The food is thus matured for the whale-field of the Azores, where this huge denizen of the seas flourishes in colder waters amidst the abundance so provided.

Our author describes yet other peculiarities of this wonderful current. Its waters are found to be warmest at or near the surface, cooling gradually downwards, so as to render it probable that there is a layer or cushion of cold water between them and the solid earth which lies below. Again, the surface of the stream is shown to be not strictly a plane; but having its axis or central portion raised somewhat higher than the level of the adjoining Atlantic; thus giving it a sort of roof-shaped outline, and causing the surface water to flow off on each side. The existence of such surface current has been proved by boats floated near the centre of the stream, which drift either to the east or west, according to the side of the axis on which they may be. This curious fact has been attributed to the central waters of the current being warmest, and therefore of least specific gravity. It may be so; but we cannot altogether discard another physical cause; viz. the enormous lateral compression exercised upon the stream by the ocean waters through which it forces its way; tending to heap it up towards the axial line. Those who have beheld the wonderful spectacle of the Niagara River, four miles below the Falls, -- so urged and compressed into a narrow ravine that the middle of the stream rises twelve or thirteen feet above the sides, - will be able to conceive this hydrodynamic influence, even on the wide scale of operation which we have now before us.

There is some evidence that the waters of the Gulf-stream, when emerging from the Caribbean Sea, are salter than those 
of the Northern Atlantic through which they flow. But as the difference scarcely exists half a per cent., we hesitate in believing, with Captain Maury, that this greater saltness is the sole source of the deep blue colour they assume. We receive, too, with distrust his speculations on what he considers the probable 'galvanic qualities' of this great stream. We have little doubt, indeed, that the electrical element, pervading in one or other of its forms the whole material world - giving motion and change to masses as well as molecules, and evolved or altered itself by every such motion and change - may have some concern, as cause or effect, in the natural phenomenon before us. But we perceive at the present time so much tendency to make use of this great power as the basis of vague and fruitless speculation, that we are always suspicious in the outset, when we find its agency invoked to solve a physical problem. In the present case we see no especial reason for having recourse to it. The physical conditions of the Gulf-stream - its definite direction, its force, its temperature, its saltness, its relation to Atlantic winds and storms, and its tardy intermingling with the mass of ocean - may be referred, with more or less probability, to other natural causes in certain and constant operation. We cannot exclude electricity from the number, but we must not invoke it on the slender evidence which our author places before us.

These considerations lead us to the theory of the Gulfstream; a matter on which a good deal has been written; and speculations put forward on very insufficient proof. Such is, the early opinion that it owes its origin to the river waters of the Mississippi, forcing a sea current before them out of the Gulf-basin - an opinion at once refuted by the utter disproportion between the alleged cause and the observed effect. It would, in fact, be the case of 300 volumes of water put into rapid motion by one volume only-such, 
according to Livingstone's careful estimate, being about the proportion of the gulf to the river stream. Another hypothesis, again, to which the names of Dr. Franklin and Major Rennell give some sanction, assigns a higher level-a heaping up, as it were, of the waters in the Gulf of Mexico, in effect of those forced into this great basin by the tradewinds of the Atlantic - thereby giving to the Gulf-stream the character of an immense river descending from this higher level to a lower one. Captain Maury suggests, we think, valid objections to this hypothesis; and even contends, from the relative depth of the stream in the Narrows of Bemini and of Hatteras, that instead of descending, its bed represents the surface of an inclined plane with a descent from north to south, up which plane the lower depths of the stream must ascend. We are bound to say that he does not replace, by any complete theory, the opinions which he thus annuls. Nor is it, in truth, easy to frame one which shall meet all the conditions required, seeing the present imperfect state of our knowledge of the mutual influerfee and action of the mighty agents concerned in such phenomena-the ocean, the atmosphere, the rotation of the earth on its axis, the change of seasons, the tides, the heat and cold of different regions, and possibly other influences, of the obscurity of which we have just spoken. All who are familiar with the science of Hydrodynamics and the theory of waves, know that these subjects involve problems requiring for their solution the highest mathematical power, based upon the most exact experiment and observation; questions which have exercised the genius of Euler, Lagrange, Poisson, Prony, Cauchy, Weber, Venturi; and in our own country, of Brindley, Smeaton, Young, Scott Russell, and others. The theory of the Gulf-stream has close connection in many points with these high problems, while at the same time complicated by its manifest relation to the great natural agents just named. 
We must, then, excuse in our author his somewhat desultory view of a phenomenon, of which no single or simple explanation can rightly be given. It is certain, from the permanent characters of the Gulf-stream, that he is correct in treating of it as part of a great circuit of waters in the Atlantic, determined and directed by natural causes of constant operation. One main influence we may presume to be, the tendency of the polar and equatorial waters to exchange and equalise their temperature by currents flowing at different depths through the ocean; - a condition certain to exist, and well illustrated by the phenomena of those constant or periodical winds, which fulfill a similar object, by maintaining the needful balance of temperature in the great atmospheric sea around us. Nor is this reference to the trade winds one of analogy only. We cannot doubt that they are concerned in keeping up the flow of those vast equatorial currents which, traversing the Atlantic from the African coasts, are pressed into the Caribbean Sea and Mexican Gulf on their southern side; and sweeping round this great basin and its islands, are mainly discharged through that narrow passage between Cuba and Florida, where the name of the Gulf-stream is first attached to the curront. All its characteristics may best be explained under this general view. If a mass of waters be constantly thrown into the Gulf, a mass of waters must as constantly find exit from it. If the exit be narrow, the force of the stream will be proportionally augmented, by the unceasing pressure from behind; rendering it powerful aud persistent enough to cleave the waters of the ocean; making a return path for itself to the more northern parts of the eastern hemisphere, and carrying thither the warmth derived from the eternal summer of the equatorial seas.

We can have little doubt that this outline conveys the true theory of the Gulf-stream; associating it broadly with those 
great currents of circulation over the globe, which we know must be the certain effect of differences of temperature, but which may in part also depend on the diurnal rotation of the earth, affecting the rate of motion and direction of such currents as they flow through different latitudes. The Arctic current setting into the Atlantic from Baffin's Bay, and transporting huge icebergs to be dissolved by the warmer seas of the South, is well known as a branch of one of these circuits. The existence of a similar circulation of waters in the Pacific - the other great ocean which stretches from pole to pole of the globe - though less defined in its details, comes in confirmation of this view. It is more directly corroborated by the old experiment of casting bottles into the sea containing dates of place and time; which transported in silent, slow, but certain course, give information to watchful observers on distant seas or shores. These mute interpreters of natural phenomena often render better service to science than the thoughts or theories of man. The chart drawn up by Admiral Beechy, representing the tracks of more than a hundred bottles, shows that all the equatorial waters of the Atlantic tend westwards towards the Mexican Gulf, to issue thence in the Gulf-stream. Those thrown overboard in mid-ocean, or on any part of the African coast, have been found after a certain lapse of time either in the West Indies, or on the British shores, or floating in the course of the Gulf-stream between. There is even reason to believe that some of these bottles have been discovered on their second circuit; arrested probably on the coasts of Spain by the drift southwards, carried along the African coast into the equatorial seas, and thence againacross the Atlantic to the Gulf of Mexico. The first among the valuable plates appended to Captain Maury's work, clearly shows the course thus indicated, and illustrates the whole scheme of the mighty currents we have been describing. 
Whenever a circuit of waters is thus formed we have every reason, from tidal and other analogies, to look for an intermediate or central space, comparatively calm and motionless. And such a space is actually ascertained to exist within this great Ocean whirlpool. The 'Mar de Sargasso,' as the Spanish navigators termed the central portion of the Atlantic, stretching westwards from the Canaries and Cape Verd Islands - a surface fifteen times greater than that of Great Britain may be described as a vast stagnant pool, receiving the drift seaweed, which the surrounding currents fling into it, and generating on its calm surface what has been well called 'an oceanic meadow' of seaweed, the fucus natans of botanists. It is in this tract of sea that we find such wonderful species of fuci as the Macrocystis pyrifera - having stems from 1,000 to 1,500 feet in length and but a finger's size in thickness, branching upwards into filaments like packthread. This vast domain of marine vegetable life is the receptacle, as indeed are the waters of the ocean generally, of an equal profusion of animal existence - from the minute luminiferous organisms, which (to borrow Humboldt's phrase) 'convert every wave into a crest of light,' to those larger forms of life, many of which derive nutriment from the water's alone, thus richly impregnated with living animal matter. Reason and imagination are equally confounded by the effort to conceive these hosts of individual existences - cette richesse effrayante as Cuvier terms it-generated or annihilated at every passing instant of time. No scheme of numbers can reach them, even by approximation; and science is forced to submit its deductions to the general law, that all the materials of organic life are in a state of unceasing change, displacement and replacement - under new forms and altered functions - for purposes which we must believe to be wisely designed, but which transcend all human intelligence.

It is interesting to possess a record of this Mar de Sargasso 
from the pen of the great mariner who first traversed it on his way to the discovery of a new world. In a letter written by Columbus in 1498, he relates that in each voyage from Spain to the Indies, he found, about 100 nautical miles to the west of the Azores; a wonderful change in the aspect of the ocean; so sudden, too, that he uses the word raya to mark the line of boundary. The sea became at once calm and still, scarcely even moved by storms; but so thickly and strangely matted over wth seaweed, as to suggest instant danger to the ships from running upon shoal banks. Nearly four centuries have elapsed since these phenomena were present to the eager and observant eye of Columbus; and they still continue as they then were. The same currents sweep round the basin of the Atlantic; the same stagnant and weedy sea still exists within the circuit of waters thus formed. How changed, meanwhile, the aspect of man's existence on the shores which bound this ocean; and how certain the greater changes during the ages which lie before us! Many of these changes, and such as may count among the mightiest now in progress, are due to the Atlantic itself, and to that permanence of its physical characters which we have been describing. Not only has it served to the intercommunication of the two hemispheres, but it may almost be said to have created the Western, by the tide of human emigration carried across from the old world to the new. Some of the greatest problems in government and social existence are awaiting their eventual solution in the races thus transplanted; and especially in the powerful nation, our own immediate offspring, established on the wide and fertile continent of the West.

We cannot touch upon this vast topic of human transit over the Atlantic, whether for commerce or migration, without recurring once more to the history of the Gulf-stream. Though in practical navigation its influence must have been often felt, 
yet this fact was scarcely recognised or distinctly recorded before the time of Franklin, whose sagacity, applied to certain special cases, showed him at once the value of a more exact knowledge of all belonging to this great current. One of these cases is curious enough to deserve mention. When in London in 1770, he was consulted as to a memorial sent from Boston to the Lords of the Treasury, complaining that the packets from Falmouth were generally a fortnight longer in reaching Boston than common traders from London to Rhode Island, a passage fully 300 miles longer. Captain Folger, a Nantucket whaler, who happened to be then in London, was questioned by Franklin, and furnished him with the true explanation. The Rhode Island traders were acquainted with the Gulf-stream, and kept out of it. The captains of the English packets, from ignorance or carelessness, or possibly seduced by the more genial temperature of this southern course, ran their vessels into the current and against it; making a difference in some parts of their voyage of not less than fifty or sixty miles in the daily run, besides the loss incurred from sailing in a lower latitude. Dr. Franklin made Folger, whose experience taught him to avoid a stream in which whales are never found, trace out on a chart the course of this ocean current, had it engraved, and sent copies to the Falmouth captains. These gentlemen, wedded to their old ways, or perhaps despising their informant, took no notice of the suggestion and went on as before.

Franklin was also the first to indicate the temperature of the Gulf-stream, as a valuable aid to the navigation of the Atlantic, especially on the American coasts; the dividing line between the warm stream and the cold waters of the ocean which hem it in, being so precise as well as constant, that the longitude may often safely be inferred from it. Captain Maury affirms and, we doubt not, with truth, that 
this dividing line never changes its position in longitude as much as mariners then erred in their reckoning. He gives us also a very curious account of the relation of the Gulfstream to the storms and hurricanes of this ocean, to which is due their frequent character of rotatory storms or cyclones; a name well adapted to the remarkable phenomenon so described. One passage here we will transcribe from our author.

I am not prepared to maintain that the Gulf-stream is really the 'Storm King' of the Atlantic, which has power to control the march of every gale that is raised there; but the course of many gales has been traced from the place of their origin directly to this stream. Gales that take their rise on the coast of Africa, and even as far down on that side as the parallel of $10^{\circ}$ or $15^{\circ}$ north latitude, have, it is shown by an examination of log-books, made straight for the Gulfstream:- joining it, they have then been known to turn about, and travelling with this stream, to recross the Atlantic, and so reach the shores of Europe. In this way the tracks of storms have been traced out and followed for a week or ten days. Their path is marked by wreck and disasters.

One such storm commenced more than a thousand miles from the Gulf-stream, made a straight course for it, and travelled with it for many successive days under the conditions of a whirlwind or cyclone. A fearful disaster, due to one of these hurricanes, occurred in 1853, to the steam-ship 'San Francisco,' carrying a regiment of United States troops from New York to California. Overtaken by the storm in crossing the Gulf-stream, 179 souls, officers and men, were swept overboard and perished. In this case, the knowledge possessed of the stream, its limits, direction, velocity, \&c., greatly aided what was done for the discovery and relief of those who survived. The import of these and similar facts to the future guidance of Atlantic navigation will readily be understood. It may be hard to account for them in theory, but their practical value cannot be doubtful or mistaken. 
Intending, as we have said, to confine our remarks chiefly to that ocean on which Captain Maury himself best loves to expatiate, we shall follow him more cursorily through the other parts of his volume. Three chapters of his work relate to the Atmosphere, in its various connection with the physical geography of the sea; as expressed by the phenomena of winds, of evaporation, of rains, of temperature, of fogs, and of electrical changes; - a vast subject, and not less complex than vast. Multiplied though all its records have been of late years, and made more minute and accurate as well as numerous, Meteorology cannot yet take its place among the exact sciences. We have just named some of the topics it includes; but there are yet others, which mix with and complicate all the results of observation. The weight of the air is one of these; an element involved as effect or cause in almost all other atmospheric changes, and deeply concerned in any theory of the winds. Again, we have those conditions of Electricity, which are expressed by the wonderful phenomena of magnetism acting through and upon all parts of the globe, solid, fluid, and aërial; and brought before us under a new aspect by Professor Faraday's discovery of the magnetic properties of oxygen as modified by heat. Even that other subtle element of Light - if indeed it be another and separate element-may in some sort affect the atmosphere, through which its action is transmitted to the earth and ocean below. As associated with, or, according to our recent philosophy, converted into heat, there can be no doubt of this influence. But the marvellous results which science has obtained from the chemical actions of light, justify the belief that other analogous effects may exist, though yet hidden from human observation. If electric states of atmosphere are able to convert oxygen into Ozone, light, in its different degrees of intensity, cannot well be supposed without influence, even on the inorganic parts of the aërial medium 
through which its passage lies. We know well its wonderful power in evoking the organic life, with the germs of which the atmosphere everywhere teems; and there is even reason to believe that this influence extends to different depths of the sea, concurring with other causes to define those successive strata of animal and vegetable life, which are so curiously attested as the result of the marine dredgings and soundings directed to this object.*

We deviate thus far from our direct subject, merely to point out the singular complexity of those elements and relations, which make up the history of atmospheric phenomena, whether on ocean or land. Such, and so close are these relations, that scarcely a change can occur in any one of them, without altering or disturbing more or less the balance of all. Science is now seeking to decipher these phenomena; and to obtain both more exact results, and knowledge of the relative influence of the causes producing them. But longer time and wider averages are required to this end; and meanwhile what becomes most needful is a patient and precise observation on all parts of the globe, and in all climes and seasons; aided by such an amount of provisional theory as may serve to the guidance of research, and to bind facts together until they can be submitted to the governance of general laws.

These considerations may mitigate a criticism to which Captain Maury's work is liable here, and indeed more or less throughout. He theorises too largely and hazardously, and

* In speaking of the influences of Light upon organic life, even in the depths of the sea, we would advert for a moment to those singular fossil crustaceans, the Trilobites. Found as far down as the Lower Silurian Rocks - the earliest known date of animal life, and far beyond all human measure of time-the eyes of these creatures are so well preserved in some specimens, as to show some four hundred spherical lenses composing this organ; indicating thereby not merely similar conditions of light, but also that the waters of the sea at this epoch were as transparent as in our own day. Conclusions of this kind, thus obtained and applied, are strikingly characteristic of modern science. 
does not clearly separate the known from the unknown. His volume is replete with ingenious suggestions, but they are not methodised enough for the uses of the common reader, who will probably rise from the Chapters on winds and atmospheric currents, his head confused by a whirl of facts and theories and questions, as fleeting as the very air of which he has been reading. It must be admitted that this subject of the Winds of the ocean - whether permanent, periodical, or variable - is one of very difficult and intricate kind. The differences of temperature between the tropical and arctic regions, and the influence of the earth's diurnal rotation upon the currents of air thus produced, afford us a rational theory of the trade-winds. The periodical monsoons of the Indian Ocean, though depending in part on the same causes, are singularly modified by the proximity of great continents, islands, and mountain ranges; and though well known to practical navigators, their character is less certain and their interpretation more obscure. Still slighter is our knowledge of the variable winds, in those narrower seas where the influences of the land become predominant over those of the water; phenomena in which we in England have great practical concern, but to which it is at present difficult to give any systematic form.

The Barometer, though less certain here than on the wide Oceans of the globe, is still the instrument on which we may best depend; and the recent extension of its uses (aided by the electric telegraph), around the English coasts, will every year save hundreds of lives among the sailors and fishermen of our shores. It must however be kept in mind that our direct knowledge of the winds is derived from the lower strata of the atmosphere only. The aspects of clouds often show to the eye different or opposite currents at different heights : observations in balloons testify the same thing. Beyond this our conclusions, though inferential only, warrant us in be- 
lieving the upper regions of the atmosphere to be traversed by currents of lesser density, but as determinate in space, time, and direction, as the winds which sweep periodically over the surface below. The general equilibrium is ever maintained; and this can only be effected by circuits and counter currents at different heights, according to the differences of temperature of each. The inference here approaches to a demonstration of the fact, though not reaching it by actual observation.

We cannot speak with the same assurance of a speculation, which however is sanctioned by eminent names, viz. that the more sudden and violent gales of wind, the tornadoes and whirlwinds of the seas, are due to the upper currents of air bursting abruptly into those of lower level; and by their different direction of movement, different temperature, and possibly difference of electrical state, begetting the various phenomena of storm on the ocean beneath. No better theory has yet been proposed for these sudden hurricanes; and in default of such, we must admit it as one of the many meteorological questions open to future research.

We should abuse the patience of our readers, were we to dwell longer on the subject of atmospheric currents thus encircling the globe, and, under their various conditions, aiding or endangering the labours of man on the seas. The only remark we have to add respecting Captain Maury's chapters on the atmosphere is, that he does not sufficiently allude to the influence of the variable weight of this great aërial ocean upon the ocean of waters below. Those who have attended to the phenomena and theory of the Seiches in the small basin of the Lake of Geneva, or witnessed the frequent and abrupt oscillations of a forty-feet water barometer, will be able to appreciate this element of unequal atmospheric pressure, as applied to the great watery surface of the globe. Nor do we find any allusion by our author to 
the singular fact recorded by Sir James Ross, of the permanently low barometric pressure in high southern latitudes; or to the curious observations of Professor Airey and Mr. Birt, on the periodical rise of the barometer in the course of every month to some point above $30^{\circ}$; suggesting the notion of great atmospheric waves, ruffled by smaller waves in the intervals between. We must look to the future for a solution of these, and numerous other difficulties in meteorology, which are beyond the reach of any tables or averages yet obtained. Many of these phenomena may be best studied under the equator, where there is little variation in the sun's meridian altitude; where the zone of observation is symmetrically related to each hemisphere; and where the diurnal fluctuation of pressure is so regular, that the time may generally be determined within 15 or 16 minutes by the barometer alone.

The 'Depths of the Ocean,' and the methods employed to determine them, form an interesting chapter in the volume before us. Until a very recent time these methods were so far imperfect that, though numerous soundings were made into the more profound depths which sailors call Blue water, it could seldom be affirmed 'that fathom line had truly touched the ground' in these abysses of the sea. In the Southern Atlantic, more especially, results were given as obtained by British and American officers, which indicated depths varying from 26,000 to 50,000 feet, or from 5 to $9 \frac{1}{2}$ miles; and in several of these instances without any assurance of the plummet having reached the bottom. Here, in fact, lay the uncertainty of the whole process. Undercurrents might intervene, turning aside a slender thread and insufficient weight from the right line of descent; or, if allowing the weight to touch the ground, still acting upon the bight of the line, so as to cause it to run out too far from the reel in the vessel above. 
We owe a better system of soundings to the active ingenuity of our American brethren on the seas. It was first decided that the twine used for this purpose must be of stronger texture; so as to bear the weight of at least 60 pounds freely suspended in the air. This sounding twine is divided by 100 fathom marks. The weight employed is a simple cannon ball of $321 \mathrm{lbs}$. or $68 \mathrm{lbs}$. weight, so appended, that on touching the ground it is detached from the twine; leaving, however, to reascend with the latter an ingenious little apparatus (the contrivance of Mr. Brooks of the United States Navy), which gathers and brings up specimens from the bottom of these deep recesses. Experiments made with lines thus constructed, have furnished a scale of the average time of descent for different depths; exact enough to tell pretty nearly when the ball ceases to carry the line out, and when, therefore, the depth is truly determined.

The result of these improved methods has hitherto been to indicate a lesser depth than was inferred from previous soundings. The greatest hitherto ascertained is in the North Atlantic, on the southern edge of the Banks of Newfoundland; where the ball touched the ground and parted from its line, at about 25,000 feet, or nearly five miles below the surface. Yet if Laplace's calculation of four miles as the mean ocean depth be correct, there must exist spaces with far deeper soundings than this. And such in truth we may expect to find, when navigators apply their present resources to fathom those other vast oceans, where the line has rarely been sunk for the purposes of science only; and where the phenomena of coral isles and volcanoes, show conditions of deep subsidence as well as elevation from physical actions occurring in the interior of the globe. The time may come, but yet is far distant, when we shall be able to map this great submarine territory with some approach to truth; and in so doing obtain, perchance, a further insight 
into those wonderful changes, paroxysmal or gradual, which the outer surface of the earth has undergone in the course of ages, from central actions hitherto reached by conjecture alone. Knowledge need never be despaired of from any source, however seemingly remote, where the connection of the physical sciences is becoming so intimate in all its parts. A single instance may be given as peculiarly belonging to this Ocean of which we are treating. In a remarkable memoir by Professor E. Forbes on the ' Connection between the existing Floras and Faunas of the British Isles, and the geological changes which have affected this area,' we find denoted, amongst other curious local relations of certain British species to those of the nearest opposite continents, the singular case of identity of several species in the Southwestern Irish Flora, with species found not nearer than the mountains forming the northern coast of Spain. On various grounds Professor Forbes concludes - and he was not a rash speculator in science - that the British Isles acquired this connection of their Flora and Fauna with that of neighbouring lands, by immigration of species before the area they now occupy was severed from the greater continent. The specialty of the Irish case as to distance does not deter him from following out this conclusion. Boldly, but not without much show of reason, he draws a line of ancient continent across the Bay of Biscay and yet farther westwards into the actual Atlantic. Geology tells us of numerous changes and alterations of land and sea, similar in kind and still vaster in extent. Those changes which we may suppose to have visited Britain, though far removed from man's knowledge, are comparatively recent in the history of the earth presumably of later date than what has been called the Meiocene epoch. It might seem as if a sort of specious reality were thus given to the ancient fable of the Atlantis : 
but no history of time will serve us here, and this legend must be left in its old obscurity.

We cannot quit this topic of the depth of the Atlantic, without referring to one matter connected with it, far surpassing in grandeur of idea any fable of the imagination we mean the Atlantic Electric Telegraph, so recently approaching its completion. The scheme, if not originating in a series of soundings across this ocean, was at least matured and directed by them. These soundings disclosed the existence, between Newfoundland and the western coast of Ireland, of a sort of plateau forming the bed of the sea, at a depth in no place exceeding 2,070 fathoms. This point of greatest depression is very nearly equidistant from Valentia and St. John's, the assumed eastern and western termini of the telegraph. The actual distance between these points is 1,900 statute miles; of which about 1,500 miles intermediate between the dips from each side, and named by Captain Maury the 'Telegraphic plateau,' afford a soft and singularly equable level. It is seemingly a surface of calcareous rock, covered in great part with a layer of microscopic tropical shells, and well adapted in every way to receive the wonderful instrument of human intelligence committed to this submarine bed. It was surmised, and not without show of reason, that these very materials, forming the bottom of the plateau, might furnish a coating of natural concrete to the electric cable; adding to its stability of position, lessening the chances of injury from the elements around, and possibly also affording a more perfect means of transmission of the electric action itself.

We cannot afford space, and it would be alien to our subject, to dilate on this extraordinary project; successful for a moment, then suddenly annulled, by causes which we apprehend will ever endanger submarine lines thus long and deep. We are not yet able to conjecture whether the 
attempt will be renewed by any other line, or in any other form.* But we may well venture to affirm that delay is a lesser evil in this case than hasty and premature execution. Besides those risks to the coating of the wires and to their perfect insulation, which may depend on the depth and other less known conditions of the Ocean bed, we are obliged to admit that there are still difficulties in the theory of electric currents, and of electric actions by induction, which very especially apply to the case of long submarine lines for telegraphic use. Some of the several conditions which were suggested as likely to retard or otherwise impair a current thus prolonged, have been made the subject of careful experiment by those most competent to the research. $\dagger$ This enquiry is still in progress, including especially all that relates to the thorough insulation of the wires. So subtle is the agent they are called upon to transmit, and so liable to escape from its artificial channel, that a breach hardly perceptible to the eye might be fatal to the integrity of a line, otherwise perfect, across the Atlantic. We cannot better denote the beauty and ingenuity of the experiments applied to this object, than by stating that the electric current itself has been made to indicate, within certain limits, the point in the submarine wire where any fault or injury to insulation has occurred, even though this be hundreds of miles from the land terminus of the line. Scarcely indeed is

* Another telegraphic route to America has since been suggested (we are now writing in 1862) by the Faroe Isles, Iceland, Greenland, and Labrador; and partial surveys were made in 1860 to determine its feasibility. Such route would aroid any sea line, equalling in length that from Valentia te Newfoundland; but would incur various difficulties of execution and of mantenance in working state, greatly diminishing, if not annulling, the advantage thus gained.

$\dagger$ In connection with the experimental researches directed to the improvement of the submarine telegraphic cable, as well as to the best methods of ' rapid signalling,' by this great instrument, we must especially mention the name of Professor Thomson, of Glasgow, than whom no one is better fitted by his various acquirements, to solre the difficulties of the problem. 
there any distance to which this principle of detection may not be applied, supposing that the defect is limited to a single spot only. The discerning of a fault, however, and even of the place of its occurrence, is much easier than its repair; and here will be found, we apprehend, the great practical hindrance to spanning the Atlantic by a telegraphic cable. The large expense of the work demands a security for its permanence which it will be very difficult, if not impossible, to obtain. The evidence derived from other and shorter submarine lines does not yet suffice to give this guarantee.

As to the practical results to the welfare of the world, and more especially of England and America, from the contingent completion of this singular work, we are not altogether persuaded by the current phraseology of the day. It is easy to affirm that whatever gives fresh facilities to human communication, is productive of good, and difficult perhaps to disprove the assertion. But in so stating the matter, we must keep in mind that it is the speed of intelligence only which is here chiefly in question. Doubts may suggest themselves, whether the farthing-a-pound fluctuations in the price of cotton deserve a daily transmission across the Atlantic; especially as the same means may be used to tell the fact almost simultaneously to every Liverpool broker or Manchester manufacturer. The demand for any particular article of traffic, whether raw or manufactured, is rarely so sudden or impetuous as not to be able to await transmission by the next steamer. A criminal fugitive may be arrested at the moment of landing, by his description outrunning him on the ocean, or relatives be brought sooner to a distant bed of sickness; but the simple tidings of friendship or family affection will not trust themselves to be interpreted by the vibrations of a needle, and the translations of a hired pen. Even in the more serious matters of diplomacy, we may doubt whether 
the old-fashioned pauses in intercourse were not as salutary as these instant communications; giving longer time for passions to subside, and for first impressions to ripen by reflection; and preserving to the diplomatist a responsibility, equally essential to his own honour and to the interests of the country he represents. In time of war (absit omen!), the advantages obtained may be more numerous and certain. But even here we must balance them against the effects of lessened responsibility to those engaged in the duties of actual command; and of faulty information at a distance superseding the better judgement derived from local knowledge. We are aware however that there is a double aspect to all these points; and without pressing further any such ambiguous presages, we shall be ready to join in the general gratulation on the success of an undertaking thus wonderful as an effort of human genius and power; and destined, we trust, to link together still more closely in amity as well as intercourse, the two great nations already having kindred in origin, language, and common liberties.

We have occupied so much space with the various topics, that our notice of the other parts of Captain Maury's volume must be a very limited one. In a chapter on the 'Salts of the Sea,' he propounds his views, and perhaps with some exaggeration, as to their influence in creating ocean currents by the different specific gravity of strata of water differently charged with salt. To the curious question regarding the origin of this saline matter, (amounting to three and a half per cent. in the average of all seas,) he answers that it was thus when the Ocean was created; that no washing down of salts by rivers can adequately explain the phenomenon, and that the 'Christian man of science' may rest his belief, on the absence of any proof from Scripture that the sea waters were ever fresh. Even accepting the conclusion as probable, we must repeat our remonstrance against this mode of 
stating it. The question in itself is one of much difficulty, and we can see no evidence that it is ever likely to go beyond presumption. The uniformity in the quantity, quality, and proportion of the saline constituents, and the fossil animal remains of ancient Salt seas, now found many thousand feet above the ocean surface, would seem the strongest proofs of identity of state from the beginning. The presence in all sea-water, though in most minute proportion, of those singular elements (or what are provisionally called such) Iodine and Bromine, becomes a special part of this argument, and cannot be neglected. We do not venture to cite to the same effect the recent discovery of Silver, as another ingredient; since further experiments are needed to attest its universality $^{*}$; and perhaps we must yet postpone that still later discovery (1861) of Lithium in sea-water, by the new and marvellous method of the spectrum analysis. But all these researches show in their results the complex and wonderful nature of that ocean-fluid, which wraps round so large a part of the solid globe.

In treating of the various ocean temperature, and its influence in producing currents, we do not observe any notice of that singular and important discovery which we owe to Sir James Ross ; viz., the existence of a stratum of invariable temperature (39 $\frac{1}{2}^{\circ}$ Fahrenheit), pervading the ocean from north to south, and represented on each side the equator by a similar

* The discovery of silver in sea-water by Malaguti and Durocher, is curiously confirmed by certain experiments of Mr. Field, showing the presence of silver, even to the amount of seven ounces to the ton, in the copper sheathing of ships after long-continued voyages. These observations are related in a paper read to the Royal Society some months ago.

We may notice here the curious experiments of Professor Chapman of Toronto, as to the comparative rate of evaporation from salt and fresh water. They show that the greater the proportion of salt, the slower the evaporation; and that water containing the same percentage as that of the sea, loses in 24 hours not quite half as much as fresh water. This fact gives some support to Mr. Chapman's theory, that one great use of the salt in the Ocean is that of regulating and controlling the evaporation ever going on over its vast surface. 
and very curious curve, depending on the superficial heat or cold in different latitudes. At the equator the depth of this level of constant temperature is 7,200 feet; - in latitude $56^{\circ}$ it is at the surface; - in the Arctic regions it descends again to 4,500 feet; the temperature in each case being invariably the same (that is $39 \frac{1}{2}^{\circ}$ ), below the level of these several depths. The value of such observations to every theory of submarine currents will readily be perceived.

To the domain of the North Atlantic belong those Arctic Seas, which, stretching northwards on each side of the projecting continent of Greenland, lead on the western side to the ice-bound recesses of Baffin and Hudson Bays - on the eastern to Iceland and Spitzbergen, possibly even wrapping round the Pole itself; though we are yet ignorant (as in the case of the southern Pole also) whether this great axis of our globe terminates in land or water. These northern seas have been during the last forty years the scene of those bold and perilous enterprises of English navigators, which give us so much to admire; alloyed by one great calamity which we can never cease to deplore. The problem of the North-west passage solved, and its utter uselessness ascertained, the stern regions of the Northern Coast of America may wisely now be left to their primitive solitude. If other attempt be made hereafter in Arctic discovery, we would fain see it take a direction to the east of Spitzbergen; - a route hitherto unattempted by English navigators, and which would be aided by means wholly unknown in the earlier days of Arctic discovery. We confess our desire that the nearest approach of man to the pole of his planet should be due to English enterprise and perseverance.

In a chapter on 'Ocean Routes' our author gives some graphic narratives of that racing on the high seas, which, if it be the pride and profit of modern navigation, is also oftentimes to be accounted its folly and peril. The struggle for 
superiority, whether by sail or steam, is still almost exclusively between England and the posterity of England in America; the two great commercial communities of the world. Though the Indian and Pacific Oceans form part of the scene of contest, the Atlantic is the arena where science and skill, aided by abundant capital and incited by strenuous emulation, have achieved results which only a quarter of a century ago would have been deemed impossible. These results are too well known to need relation here; but we may notice briefly one or two facts, illustrating the wonderful changes now in progress in commercial navigation. We should scarcely err in stating the average duration of long ocean voyages - as those to or from China, Australia and India, performed by the best sailing ships - at barely half what it was at the period just named. Among the causes concerned in this great result must first be noted, the improved construction and fitting of ships; and especially with reference to what Mr. Russell has called the wave principle of construction; or, in other words, the form of least resistance of a solid moving through water. Connected with this, and in practice now applied to the same end, is the direct relation ascertained to exist between the length of the vessel, and the speed it is capable of attaining. But beyond these altered conditions of the vessel itself, comes in the enlarged and more exact knowledge of the seas it traverses; of the winds and currents, the shoals and depths, and the various other physical phenomena of the ocean, which have been brought to the aid of practical navigation, and to which we have already so copiously referred. To the combination of these causes, and the record of the tracks and times of many hundred voyages, upon methods which Captain Maury has done much to enforce, we owe those feats of seamanship, which have brought India and our Australian colonies within ten or twelve weeks of England, and made the circumnavi- 
gation of the globe as frequent and familiar as was once the passage across the Atlantic.

We have here been speaking of sailing vessels, but Steam navigation has its own peculiar history; including not only these several improvements, but others also which depend on more perfect machinery and a higher class of engineers. Though steam has now spread its dominion over the globe, the Atlantic is still the sea where it puts forth its greatest powers. The several lines of Mail Steamers across this Ocean, and more especially those familiarly known as the Cunard and Collins lines, have reached a degree of speed and regularity, which it would be hazardous to say may not hereafter be surpassed, but which will ever be a monument and mark of human prowess, in bringing the physical elements under subjection to the uses of man. It is no serious disparagement to the second of these lines, to say that it has lost the superiority for a short time gained in speed over the Cunard line of English steamers. According to an American statement now before us, we find that, during the last year, the average of twenty-five passages from Liverpool to New York, by the American steamers, was 12 days $16 \frac{1}{2}$ hours - by the English steamers, 11 days 22 hours: of passages from New York to Liverpool, by the American vessels, 12 days 8 hours, - by the English, 11 days 3 hours. Many circumstances concur to this result; chiefly perhaps the consummate discipline of the English vessels in every department of their service. But the rivalry we regard as an honourable one; and it may yet be maintained, advantageously to the interest of both nations.*

It is however, as we have said, a rivalry not without risk. In seeking for the maximum of speed, safety is jeopardised

* About two years after this article was written, the competition here alluded to ceased, from the abandonment of the Collins line of steamers. That of Cunard still maintains, unimpaired, its high position on the seas. 
in all these great lines of mail steamers. Winter storms, icebergs, fogs, cyclone-hurricanes, and collisions with other vessels, are all encountered at high rates of velocity, and with many calamitous results. Experience and discipline have done much to protect against these dangers, but serious hazards" still exist; and especially those of collision, which are constantly augmenting in an ocean every year more crowded with ships, seeking to find the shortest passage across it. In these days, however, of bold design and prompt execution, there are few ills which do not bring with them the suggestion of remedy. Captain Maury, and others in sequel to him, have urged the adoption of 'steam lanes' across the Atlantic; - that is, well-defined lines of navigation of a certain width, and separate from others throughout; so appropriated respectively to vessels going east or west, that the chances of collision may be greatly lessened if not altogether removed. The width of the zone of ocean, now traversed by the mail steamers to North America, is about 250 miles. It is proposed to mark off lanes, 20 or 25 miles in width, on the northern and southern borders of this zone, as the routes respectively to be followed and adhered to by all steam vessels crossing in one direction or the other. The scheme, or some one equivalent to it, we doubt not to be practicable; and such is its obvious utility, that we as little doubt its being eventually carried into effect. The phrase of a Steam lane may somewhat startle those who are wont to associate with this word the cross roads of a midland rural district in England - the high hedges, deep ditches, and straggling cart ruts; the bushes of blackberry, hazel-nut and hawthorn; and the hundred sweet flowers and weeds which luxuriate on the hedge banks. We cannot quarrel, however, with this novel use of the term, if the object be fulfilled to which it is applied. If long lanes of ocean, 'which have no turning,' be really laid out for the safer navigation of the seas, a great 
point in maritime economy has been achieved. The very simplicity and familiarity of the name so given is a tribute to that prowess of man, which has taught him thus to mark out and pursue a fixed path through the wide wilderness of waters.

Though not having exhausted the subject of the Atlantic, either in its physical features or in its relations to human industry and power, we stop here. The points we have touched upon will show how copious and interesting a topic, under both these aspects, is the 'Physical Geography of the Sea;' and how worthy to be embodied with the other great subjects of human knowledge, which at this time enlighten and animate the world. Every year enlarges its domain; and we may fairly predict that the history of the Atlantic, written twenty years hence, will be a record of numerous physical facts, now either unknown or dimly and doubtfully understood. Whatever their particular nature, we may be certain that they will tend to illustrate that mutual connection among different branches of knowledge, to which every day is adding further testimony. 


\section{THE MEDITERRANEAN SEA.*}

[EDINBurgh Review, OctoBer, 1857.]

COPIOUS, even to excess, as is the literary labour of our age, and ever seeking new.topics or new methods of vivifying old ones, there are yet subjects to be found, either not touched upon at all, or scantily and incidentally treated without due regard to their proper value. Void places of this kind still occur in the history of men and nations; gaps which it will belong to future genius and research to fill up, by aid of the fresh materials ever accumulating around us. The laborious activity of German literature has gone farther than that of any other country, in finding such new fields and fertilising them by its industry. But others yet remain to be opened, even in the records of human events; still more in the great domain of natural history and the physical sciences. Here especially the rapid growth of knowledge has created the need of fresh divisions in every part; of altered nomenclature; and particular treatises on topics, the increasing importance of which compels their separation from others with which they were before associated.

In a recent article of this Review, we referred to the 'Physical Geography of the Sea,' as one of the many instances in which science has required and adopted this

* The Mediterrancan. A Memoir Physical, Historical, and Nautical. By Rear-Admiral William Henry Smyth, K.S.F., D.C.L., F.R.S., \&c. London, 1854.

This Article was written in great part during a royage to Syria, Palestine, and Egypt in 1857. But I may speak of it as founded on more than twenty previous voyages throughout every part of the Mediterranean Sea. 
more specific record of a particular class of natural phenomena ; and in discussing the subject we carried our limitation yet farther, by taking the Atlantic as the special exponent of those features which belong to the ocean domain of the globe. We have now before us a volume on the Mediterranean Sea; the first English work, as we believe, expressly devoted to this subject. Its coasts and islands have been separately noted and described by travellers, geographers, and historians of all countries and ages. But no one had treated, singly and especially, of the Sea washing round and amidst these lands, and reflecting their wonderful history of thirty centuries on its waters. We possessed no work delineating its peculiar physical features; - its outline, dimensions, depth, currents, winds, and other hydrographical and nautical conditions; - the configuration of its coasts, its islands, volcanoes, and the rivers which pour themselves into its great basin.

This, then, was one of the voids of which we have spoken; and it continued such, until the progress of all scientific knowledge and the rapid spread of human intercourse by sea and land, made it needful that a physical history of the Mediterranean should be written; - an object well and ably fulfilled by Admiral Smyth in the work before us. Some time has now elapsed since its publication; but intervening events have enhanced the interest of the subject; and we willingly receive his volume as the first instalment of what is due to the general history of the Mediterranean Sea.

In a mere technical sense, the Mediterranean may be described as a gulf or inland branch of the Atlantic; but in itself this Sea has a more wonderful individuality than any other on the globe. This is true as to its physical features, singly considered; - still more eminently true as respects those relations to human history which render it an interpreter of the records of past ages, and of the ancient empires which have flourished on its shores. On no equal 
area of the earth's surface have so many and such mighty events been crowded together as within this extraordinary basin. Every keel which now cleaves its waters traverses the scene of some maritime struggle or adventure of old times and earlier races of men; or skirts shores hallowed to the scholar or historian by the memory of genius or grandeur which have passed away. Empires, kingdoms, and republics, born to sway the destinies of the world, have risen and declined upon its coasts. Schools of philosophy and eloquence, to which we still recur for instruction and example; - laws and languages, which are embodied in the literature and social institutions of every later age, - had their earliest seats around this inland sea. It is difficult to touch upon the subject thus generally without becoming too rhetorical; but we hope, in dwelling upon some of its details, to show how copious and full of interest it is, and how well meriting the special attention of some writer who may make it, as a single picture, more complete and familiar to our knowledge. The events of history are best bound together by such local associations; and none more so than those of which the Mediterranean has been the scene and centre during a long succession of ages.

It may further be alleged as an argument for such a work, that the interests of England are deeply concerned in all that regards this Sea. Of late years certain foreign writers and orators, rather political than geographical in their style and spirit, have used the term of lake in describing it. The Mediterranean is certainly not our lake; nor can it, nor ought it, ever to pass under the supremacy of any one Power. But we have large insular possessions within its circuit; we hold the mighty rock-fortress, the Calpe of antiquity, which commands its entrance from the Ocean; and we crowd its waters to their very extremity with our ships and commerce. That single line of Mediterranean navigation, which ministers to 
the rapid intercourse with our Indian Empire, through Egypt and the Red Sea, is in itself an interest of primary importance to us, and never more so than at the present moment. The communication thus opened has already reached a speed and regularity of service which place it among the highest efforts of human prowess on the seas. If other and better routes be hereafter obtained through the Persian Gulf), (which is still matter of doubt), equally must we depend on the Mediterranean for a line of passage to that part of the Asiatic coast giving easiest access to the valley of the Euphrates. More recently the mail route through this Sea has been taken as the first stage to our Australian colonies; the shortest line, following the earth's curvature, between England and the great Island-Continent, on the opposite side of the globe. It is a wonderful route to a wonderful country; each attesting that national energy and power which has brought a new people into birth, and made oceans and seas tributary to the communication with the parent land. The discovery of the Australian gold-fields has doubtless quickened these results, but time would have evolved them even without this great auxiliary.

All these things are now become familiar to us; but we nevertheless specify them, because their very familiarity is apt to abate our wonder, and to dissever them from those memorials of older times and things, to which they stand in such singular relation and contrast. Nor must we forget, while speaking of English interests in the Mediterranean, those vast naval and military armaments so recently borne on its waters to the mighty struggle before Sebastopol;- an effort of concentrated power, rising with the need, and greatest at the very moment when peace suspended its further action. These armaments in their course passed along shores and through straits, every bay and promontory of which has its place in ancient poetry or history; and 
within sight of one especial spot on which the genius of a single man (for such we believe him to have been) has bestowed an imperishable fame. It might seem ungracious towards those officers who carried with them so much spirit and bravery to the battles and privations of the Crimea, to enquire too sceptically what proportion of their number were fully conscious of the objects their voyage brought into view? - how many of them saw with the eyes of history the mountains of Lacedæmon, Scio's isle, the plain of Ilium, and the crests of Ida and the Asiatic Olympus? or felt emotion in passing through those two straits, so famous in the history and poetry of every age?

We put the question designedly; but more in regret than reproach. For we are compelled to admit that no adequate provision has hitherto been made for the special instruction of those numerous Englishmen who are called by military, naval, or colonial duties to every part of the habitable globe. The greatness of our empire, and the progress of knowledge and invention in all that regards the social condition of man, make at this time a necessity of what was heretofore only a matter of expediency. In proportion to our power is the magnitude of the duties we are called upon, as a nation, to fulfill; and one of those duties is that of sending out to the defence and administration of our distant possessions, men well fitted by temper and education to discharge their functions with integrity and intelligence; - the latter, in most cases, the best guarantee for the former. It is our sincere belief, that no one is so well calculated to fulfill these conditions as the English gentleman, in the highest intellectual and moral sense of the word. But care is needful that the standard be not lowered, either in comparison with our former selves, or with the people of other countries, at a time when all things are in a state of transition; and when much exists, in the rapid intercourse of the world, and the 
various and desultory objects before men's minds, tending to lessen the force of individual character, and to disperse the energies of thought and action too widely for their highest efficiency. True genius will rise above these and other hindrances; but we are bound to provide also for those minds of a lower grade, by whom, in effect, nine-tenths of the world's business is carried on. We gladly perceive in all that is in progress around us, a growing attention to these objects, in which England has deeper interest and concern than any other State on the face of the globe. She has not only to sustain 'her precedence of teaching nations how to live,' but to maintain unimpaired her own great life - her liberties, laws, and language - and to enlarge and perpetuate their influence on ages yet to come.

We have been led somewhat away from our subject by this question as to the sufficiency of English education, in its ordinary form, for travel in lands of classical antiquity; - a question we are compelled reluctantly to answer in the negative. Notwithstanding the time given to Greek and Latin studies in the schools and colleges of England, our youth go forth - learned, it may be, in hexameters and iambics - but wanting generally in those higher classical associations which localise the history, poetry, eloquence, and philosophy of past ages, and illustrate the revolutions of men and nations by the more lasting memorials of nature. We must not be understood here as invoking any maudlin sentiment on these matters, such as too often finds its way into books and narratives of travel; - the produce rather of after-concoction at home than of honest enthusiasm on the spot. But we wish for such previous knowledge to be brought to places illustrious in the world's history as may enable the traveller, if not to investigate and discover, at least well to understand and enjoy. Much, we conceive, might be done towards this end, by combining with common 
classical instruction a more copious and vivid illustration of the lands which have given birth and subject to this part of learning; and by admitting even the physical sciences to contribute their share to such illustration. It may be urged that this would require a higher competence and more various knowledge in those to whom the act of teaching is committed. We admit the inference, but do not on this account withdraw or abate the demand to which it applies. If what we have pointed out be recognised as desirable, the means and methods of attainment are sure in these days to be found.*

The volume of Admiral Smyth on the Mediterranean has various merits, which we fully appreciate. As a writer, he is chargeable with some little eccentricities of style; but he brings to his subject great nautical and scientific knowledge, much earnestness of purpose, and the results of an active personal survey of many coasts of this Sea; and of some, especially on the African side, before imperfectly known. His work is dedicated to the veteran Admiral Beaufort; whose long labours as Hydrographer to the Admiralty have well and worthily sustained the reputation he acquired from his admirable researches on the coasts of Asia Minor. Admiral Smyth's own labours are honourably attested by a catalogue of more than one hundred charts presented to the Admiralty; and by numerous facts in relation to currents, tides, soundings, winds, and other aqueous and atmospheric

* The institution of the Geographical Society of London, and the zeal with which its objects are pursued, is well calculated to forward the object of teaching travellers where and how to travel, and what objects still remain to be fulfilled. We gladly see attached to it the names of some members of the 'Yacht Club;' - itself a national institution, peculiar to England, and admirably fitted to sustain that national vigour and enterprise on the seas, upon which our greatness as a State so essentially depends. We honour this spirit of enterprise in the recent exploit of Lord Dufferin, who, in his little yacht of 80 tons boldly entered the Arctic seas; visiting Iceland, Jan Mayen's Isle, and even the icy coasts of Spitzbergen, as far north as lat. $78^{\circ} 44^{\prime}$. 
phenomena, which make up the physical history of this great sea. We have no right to complain that the volume before us is devoted chiefly to professional objects, since these are numerous and of eminent value. But the subject admits of a wider delineation and more ample details, physical, historical, and picturesque. $\Lambda$ work comprising these, and at the same time preserving entire the unity of the picture, is still wanting to our literature.

The very familiarity of the Mediterranean in our own days has begotten a certain indifference to its peculiarities and grandeur. Looking to physical features only, it is by far the most wonderful ocean-inlet or midland sea in the world; penetrating farther into the heart of the continent than any other, and more strangely broken and diversified in its outline by gulfs, straits, islands, and inner seas. Mere verbal description does little towards illustration in a case of this kind. A map or globe must be before the eye, and the Mediterranean looked at simply and singly in its boundaries and dimensions; putting aside all local associations which disturb such general view.* It will be seen at once how singular and curious is the configuration of this vast basin; how deeply its gulfs run into the lands which surround it; and how closely they approach at its eastern end those other deep inlets of the Red Sea and Persian Gulf, which have their connection with the Oceans of another hemisphere. The eye, cast over other parts of the globe, will see nothing

* This direction will not seem superfluous to those who have watched the curious mechanical results of habit, even in the simple matter of relative position of objects before the eye. Any one may satisfy himself of it in this instance by merely inverting a map, or turning a globe into some unwonted position, when he will seemingly have before him a totally new configuration of land and sea, which it requires some time and effort to bring back to the reality of his former recollections. The experiment is worth making as a special illustration of a large class of mental phenomena, which cannot be too carefully studied as a part of psychology. 
equal or comparable to it in these physical peculiarities. Dante, whose descriptions of nature are often as exact as they are always sublime, speaks of it as -

La maggior valle in che l' acqua si spanda, Fuor di quel mar che la terra inghirlanda.*

Those, moreover, even least tutored in modern geology and the great phenomena with which it deals, will see that mighty movements and changes must have occurred here during ages far anterior to human record or human existence on earth. We shall hereafter refer to some present proofs of these remote events (whether slow or paroxysmal in kind) in the volcanoes and earthquakes which still so singularly affect the basin of the Mediterranean. Meanwhile, a superficial view will show us this sea exchanging its waters with those of the Atlantic at one extremity; at the other, nearly 2,000 miles distant, dissevered but by a low and narrow isthmus from those which belong to the Indian Ocean. Throughout the whole of this distance it divides Europe from Africa, physically and socially the two most dissimilar portions of the globe; - the 'discordanti liti,' as Dante calls them, in the very passage from which we have just quoted. The continent of Asia closes its eastern extremity; and the Asiatic line of coast, including that of the Black Sea, which may be regarded as its inner basin, stretches nearly 2,500 miles in length. The total circumference of the Mediterranean, following the line of its great gulfs, is estimated at upwards of 13,000 miles. Its area, including the Black Sea and the two Seas of Azof and Marmora, is stated by Admiral Smyth to be $1,149,287$ square statute miles. $\dagger$ These are magnificent

* Paradiso, canto ix. 82.

$\uparrow$ In some other works a much lower statement is given of the superficial extent of the Mediterranean; but this may probably depend on the omission of the Black Sea and its subordinate basins, and on the use of the geographical instead of the square statute mile: - possibly, also, on a deduction made for the surface occupied by islands. 
dimensions; and rendered more striking by its profound depth, of which we shall presently speak, and by the lofty mountain-chains which form its coasts, or rise as islands from amidst its waters.

The name of Mediterranean does not belong to the ancient history of this sea, and is not found in the earlier geographers either of Greece or Rome. To the people of Palestine it was emphatically 'The Sea,' or the 'Great Sea.' To the Greeks and Romans it was the Sea within the columns, the Mare Internum; Nostrum Mare; or still more frequently described in history and poetry under the various local names derived from adjoining people or coasts. The word Mediterranean is not found, we believe, before the third or fourth century, appropriate though it be in the sense of a general description. Other names of common currency may still be heard among the motley traders in this sea; but they are not recognised in our maps, and it is not needful to enumerate them. Those, on the other hand, connected with its great natural divisions, as the Adriatic, the Archipelago, \&c., are necessary in themselves, and sanctioned by long and familiar historical use.

These divisions are of considerable interest in the physical history of the Mediterranean. As many as seven have been suggested and defined; but we may content ourselves with denoting one, which is instantly obvious to the sight as breaking the Sea into two great, though unequal, basins; and not less strikingly marked by certain natural features, which coincide with and illustrate the simple geographical fact. This is the partition made by the long peninsula of Italy, the island of Sicily, and the projection of the African continent at Cape Bon; - leaving a passage barely eighty miles in width between the western and eastern basins of the Mediterranean. The fact thus obvious to the eye is physically expressed by the lofty ridge of the Apennines stretching along Italy to its very extremity, and re-appearing in the 
Neptunian Mountains of Sicily; and beyond this by a bar or line of shallow sea, occupying the strait between Sicily and Africa, and separating the profound depths which lie on each side of this submarine ridge. Though a part of the line be thus submerged here, as well as in the narrower breach forming the Strait of Messina, the physical fact is even rendered more striking by this submergence; and brought more closely into relation with those geological changes which have moulded the surface into its present shape and aspects. And that great subterranean forces have actually been at work in this barrier line, we have evidence equally curious and instructive, in the volcanic phenomena, present as well as extinct, which are notable throughout its whole extent; - not uninterruptedly indeed, but so connected in proximity and direction as to give them an evident relation to a common physical cause. At the northern end of the line we find the volcanic rocks of the Euganean Hills; amidst which, in the village of Arqua, stands the secluded tomb of Petrarch. Coming southwards, a long tract of extinct volcanic formations stretches through the Roman States. Yet again, farther south, lies the region of Vesuvius and the Campi Flegrei; wonderful in its present phenomena, and not less so in those which belong to ages anteceding any known history. Following the line farther, we come to Stromboli and the Lipari Isles, still emitting flames and volcanic vapours, as they are recorded to have done more than 2,000 years ago. Yet farther south, but within sight of these isles, rises the mighty cone of Etna, circled round by a vast girdle of lavas and other volcanic rocks - the theme of magnificent poetry to early times, and in the series and succession of these rocks giving record of ages far anterior to any poetry on earth. The line of subterranean fire we are tracing has still another attestation in an event of our own days. In 1831 a volcano suddenly burst forth in the mid-sea between 
Sicily and Africa; burning for several weeks, and throwing up an isle or crater-cone of scoriæ and ashes. Scarcely had this isle been named before it was again lost by subsidence beneath the sea; leaving only a shoal bank to attest the strange submarine breach in the earth's crust, which thus mingled fire and water in one common action.

These details illustrate that new science of Physical Geography which has already added so largely to our knowledge of the earth we inhabit, and which gives such certain and ample promise to future research. The same principle of illustration will carry us yet farther; for the physical history of the Mediterranean is in every part singularly blended with the history of the nations which have successively had dominion on its shores; and with the arts, literature, and social usages which have ever rendered remarkable this portion of the globe. It has been remarked by an eminent philosopher, that human culture and civilisation have generally clung to countries brought into proximity and ease of intercourse by inland seas and deeply-indented coasts. Admitting the reality of this view, the Mediterranean may be cited as a happy illustration of the fact. And in the same sense its strangely irregular northern boundary presents a striking contrast to the unbroken and riverless line of the African coast, from Morocco to the mouth of the Nile. Egypt and Carthage, indeed, grew into grandeur on this less favoured coast. But Egypt rested on the Nile and the Red Sea; while Carthage, of Phœnician origin, had little supremacy on the African continent, but much as a naval power, frequenting or subduing the European coasts and islands on the opposite side of the Mediterranean.

It would be a matter of mere technical geography to enumerate the several chains or groups of mountains which encircle and define this inland sea; - the Sierra Nevada the long African chain of Atlas - the Maritime Alps and 
the Apennines - the mountain ranges on the eastern side of the Adriatic - the great group of Greece - the vast chains of Caucasus, Taurus, and of Libanus, all closely pertaining to its physical geography. We might name Parnassus, Pindus, Olympus, Pelion and Ossa, Hymettus, CEta, Ida, Athos, Etna, and a hundred other mountains familiar to classical memory, which either rise from the waters of the Mediterranean, or are more distantly seen in coasting its shores. But, without pausing upon these, or illustrating them by the endless quotations which will crowd on the recollection of the scholar, we may remark that no sea exhibits so wonderful a continuity of lofty and precipitous coast as the Mediterranean. With the exception of the northern shore of Africa from near Tunis to the borders of Syria, and a few more limited portions of its outline in other parts, we find generally around this vast circuit a bold mountain-frontage to the waters; magnificently exemplified in the Corniche and whole line from the Rhone to the Arno; in the African coast from Algiers to Bona; in the shores of Greece, Asia Minor, and Syria; and again in the grand range of mountains traversing the ancient monarchy of Mithridates, and forming for many hundred miles the precipitous southern boundary of the Black Sea. Many portions of these Mediterranean coasts make their first swell from the sea to the height of 3,000 or 4,000 feet, with mountains behind them more than doubling this elevation.

The islands of the Mediterranean, with few exceptions, offer the same bold elevation to the eye. In none of its physical features, indeed, is this Sea more remarkable than in the isles, great and small, which rise so numerously out of its profound depths. They almost all attest in their height, abruptness, and other features those great subterranean movements in past ages, to which we have alluded, as having variously altered the relations of land and sea throughout 
this region. Sicily, the first and fairest of these isles, offers equal and endless interest to the naturalist and historian, the artist and the poet, despite those centuries of misgovernment which have passed over it. We may affirm with safety that no equal surface on the globe concentrates so many objects fitted to delight the eye and the imagination. Sardinia, Corsica, Candia, and Cyprus abound in magnificent though less accessible scenery. Corsica indeed may now be traversed (and well merits to be so), without other fear than of bad lodgings and scanty food. The traveller of this day, forgetting the petulant epigrams of Seneca, may as little heed the savage stories of the vendetta which still linger in its mountains, forests, and wild villages. Sardinia, with equal natural attractions and once counted among the granaries of Rome, offers far less facility and safety to the modern tourist; but its political connection with the freest and most flourishing of the Italian States, gives happier augury for the future. Candia and Cyprus, scantily known to us notwithstanding their ancient fame, are awaiting the changes now at work in every part of the Turkish Empire; which changes will alike affect the islands crowding the Archipelago and rendering it one of the most remarkable gulfs in the world. Amidst this labyrinth of mountain isles and lofty coasts, rich in historical recollections of every age, lies the passage towards those inner seas, where early history and fable are blended together in that vague mystery which has its charm even for those who are labouring after truth and reality. In this great gulf of the Archipelago, moreover, Grecian poetry found the material for some of its finest descriptions. No one can have made a winter voyage through its isles, without recalling those passages - among the grandest of Homer's similes, and apparently the most familiar to his imagination - where the rude rock and promontory (the $\dot{a} \kappa \tau \dot{\eta} \dot{v} \psi \boldsymbol{\eta} \lambda \hat{\eta}$ at every moment present to the eye) are pictured as beaten upon by the winds 
which rush through these narrow island channels, and by the waves of the seas surrounding them.* In witnessing such a spectacle - magnificent wherever it be - the voyager in this sea may fairly assume that the poet himself had gazed on the very objects before him; and drawn from them that noble imagery, which has become the inheritance and poetic wealth of every succeeding age.

Among the other islands of the Mediterranean, we are bound to notice the Ionian Isles and Malta, as parts of that vast and complex sovereignty which Englishmen have spread over the face of the globe. We count them among our dependencies, though they cannot justly or expediently be considered as colonies. The Ionian Isles, indeed, we should be willing to regard as a possession held in trust for some future Greek sovereignty in the Levant, better constituted than the feeble little kingdom which now bears this name though embracing but an inconsiderable portion of the Greek soil and race. If ever detached from our rule, these islands will carry with them the memorial of much we have effected, or sought to effect, for their good; an acknowledgement grudgingly made by the present generation of Ionians, but which will more frankly and truly come from their posterity.

Among the great physical features of the Mediterranean, the most peculiar are, that singular Strait or portal which forms its entrance from the Ocean; and those inner channels scarcely less remarkable, which connect it with the lesser seas washing the very foot of the Caucasian Chain, and even penetrating into the Russian Steppes. The Strait of Gibraltar, the great passage between Sea and Ocean, is well worthy of its ancient fame; and illustrates even to the eye those fables and feelings of earlier times, by which the known and un-

* I wrote this under the recollection of a stormy December voyage in the Archipelago, in a rude Greek ressel, and with imminent danger at one time of shipwreck on the rocky isle of Sarakino. 
known are ever linked together in the human imagination. The history of Hercules - that curious, fertile, and still unexplained source of so many Grecian and Oriental myths was not unnaturally extended to this narrow egress from the known sea; and the mountain columns, Calpe and Abyle, became the laborum Herculis metce, the stern barrier of human progress towards the west. The awe inspired by this mixture of myth and reality may have been purposely augmented by the tales of the Phœnician and other traders, seeking to maintain a monopoly of that maritime traffic by which they were enriched. The early voyages of discovery, to which the names of Pharaoh-Necho, Scylax and Hanno are severally attached, did but little to correct the misconceptions of ancient geography in this region; and the Atlantis and Hesperides, if not wholly within the domain of fable, are probably only vague traces of some early discovery of the Isles on the African Coast.* Even as late as Juvenal, the intense thirst for gold is exemplified by the mariner rushing into the mysterious ocean beyond Calpe to obtain it.

\section{Calpe relictâ}

Audiet Herculeo stridentem gurgite Solem.

That a barrier once existed between the Mediterranean and Atlantic, the violent disruption of which opened the present passage, was a tradition of antiquity more natural and reasonable than most of the physical speculations of the time. A Strait, contracted in one point to little more than

* The wonderful scenery of the Canary Isles, if reached thus early, might readily have suggested some of these legends, as Tasso has indicated in the finest parts of his poem, when placing the gardens of Armida and the 'Dolce prigione' which held Rinaldo captive, in one of these isles. . The description of the Canaries by Pliny (founded on the expedition of discovery sent thither by that remarkable man Juba, Prince of Numidia), is well worthy of notice, from its exact accordance with some of the most notable of their physical features at this day. 
nine miles (not half the width of the Straits of Dover), might well suggest such a conception. And though the depth of the mid-channel at the narrowest point is fully 900 feet, this must nevertheless be regarded as a sort of bar or submarine ridge, between the ocean and sea. The waters in fact deepen rapidly on each side; - so suddenly even in the Strait itself, that between Gibraltar and Ceuta where the width is twelve miles, nearly 6,000 feet of sounding line have been run out; while somewhat further to the eastward no soundings have yet been obtained. Let any one look on a map, and mark the general trending of the European and African coasts, and their peculiar apposition and opposition in the Strait itself, and he will at once surmise that this place must have been the scene of great movements and changes, involving both sea and land; and due to the subterranean forces, which have everywhere been active, in one form or another, in altering the configuration of the earth's surface.

We must speak more cursorily of those inner Straits, the Dardanelles and Bosphorus, which give access to the vast interior basin of the Black Sea - a name once obscure and fearful in report, but now become as familiar to us as the Baltic or German Ocean. These two Straits are among the marvels of the Mediterranean:- rivers they might not inaptly be called, since they afford egress by the rapid and profound current flowing through them, to the waters from nearly one-third of the surface of Europe. . The Danube, the Don, and the Dnieper all empty themselves through this channel into the greater basin below. Their waters, in passing the Straits, flow between shores every point of which has the history or poetry of former ages inscribed upon it. No passage between seas elsewhere on the globe can compare with these, either in living scenery or past recollections. Classical legends of the most remote antiquity are here blended with the record of those more real events of con- 
quest, migration, sovereignty, and revolution of which the Bosphorus and Dardanelles have been the scene at all periods; - often defining their course and progress, often suddenly arresting it. Europe and Asia, with promontories and palaces reflected from the same great stream, may be said to divide its history between them. For the last four centuries Asia and Asiatics have had dominion here, and in name and outward recognition this may continue yet longer. But virtually a change has begun, the progress of which cannot now be arrested; and which in its ultimate effect will inevitably restore to Europe its sovereignty in these straits and seas, so important in their position to the commerce and civilisation of the world. In this respect (as in many others not yet fully surmised) the recent Crimean war will bear fruits to be gathered by succeeding generations.

The depth of the Mediterranean is another of its great physical characteristics. We have spoken of the deep soundings close to the bar in the Straits of Gibraltar, and of those which occur on each side of the submarine ridge extending from Sicily to the African coast. These soundings, indeed, have only partially been made in the Mediterranean by the improved methods lately adopted in the Atlantic, and conducted so systematically in some parts of that ocean. But they suffice to indicate depths equal to the average height of the mountains girding round this great basin; and, if one particular experiment may be credited, reaching even to 15,000 feet; - a depth closely approaching the elevation of the highest Alps. This sounding was made about ninety miles east of Malta. Between Cyprus and Egypt 6,000 feet of line have been let down without reaching the bottom. Other deep soundings have been made in other places with similar results. We have not yet obtained any official account of those recently effected by the 'Tartarus' in the lines of sea between Egypt and the Archipelago; but it is stated on 
apparently good authority, that one sounding between Alexandria and Rhodes reached the bottom at a depth of 9,900 feet; another, between Alexandria and Candia, gave a depth of 300 feet beyond this. These single soundings, indeed, whether of ocean or sea, are always subject to the certainty that greater, as well as lesser depths must exist, to which no line has ever been sunk; - a case coming under that general law of Probabilities so largely applicable in every part of physics. In the Mediterranean especially, which has so many aspects of a sunken basin, there may be abysses of depth which no plummet is ever destined to reach.

This mere outline of the Mediterranean in its prominent features, establishes its pre-eminence over every other inland sea on the globe. The Baltic is another singular inlet, spreading its long and narrow gulfs into the centre of Northern Europe; but as different in its physical characters as in its relation to the history of the world. Far inferior in extent, - accessible only through shallow straits, - and with a depth which in no part exceeds 1,100 feet, its waters are brackish and tideless, its coasts and isles flat and monotonous throughout. No historical monuments, save a few of mediæval age, are found within its circuit. Ancient history indeed gathers none but the most vague and scanty records from its shores, notwithstanding that we know them to have been traversed by some of those great races whose migration from the East has so mightily affected the destinies of Western Europe. Though for a time animated by the mercantile activity of the Hanseatic League, yet until the epoch and creations of Peter the Great, when the Empire of Muscovy reached the mouth of the Neva, the Baltic had a very small share in the political or commercial interests of the European world. The revolutions of the Swedish monarchy, and the campaigns of Gustavus Adolphus and Charles XII. form the only conspicuous exceptions to this remark. 
Perhaps the nearest physical parallel to the Mediterranean might be found in the Gulf of Mexico; - detached from the Atlantic by the continuous chain of the Leeward Islands and Bahamas, and similarly divided into two great basins by the projection of Cuba between the Florida and Honduras coasts. The volcanic isles of this gulf afford a further analogy; and yet more, the extraordinary isthmus which divides the Atlantic and Pacific Oceans; - the only comparable instance on the globe to that of Suez; - singularly alike, too, in the length and outline of that vast circuit of at least 15,000 miles, by which alone navigable communication can now be made between the waters pressing on each side this narrow neck of land. Human enterprise, rioting at this moment in a triumphant struggle with all natural obstacles, is seeking simultaneously to cut a passage for ships across each isthmus. Though the Suez Canal scheme of M. Lesseps (animated perhaps by a certain political feeling, ) has already entered upon its workings, we see no reason to alter the opinion we formerly expressed, that it will end in practical failure. The high authority of Mr. Stephenson confirms us in this belief.

But we have not yet done with the physical wonders of the Mediterranean. Its volcanoes, active or extinct, and the earthquakes which are so frequent and violent within its area, merit more especial notice; expressing, as they do, those local subterranean forces which have been concerned in forming and shaping this extraordinary ocean gulf. We have already traced the singular line of volcanic action which may be said to divide the Mediterranean into two great basins, manifestly defined by the continuity of these great natural phenomena. It is needless to quote the Greek poets or historians in proof of the antiquity of the fires of Atna and the Folian Isles, since the series of volcanic rocks around them attest ages of prior eruption, of which no human record exists. Monte Somma, that strange and solitary remnant of 
the older cone of Vesuvius, tells the same tale of a time not approachable by date, and of which there is no tradition. The history of the present Vesuvius, so active in every succeeding century, begins but with the time of Pliny, and the destruction of those cities which we are now disentombing at its foot.* A similar attestation of age we have in the various extinct volcanic districts of Italy and of Sardinia. Closely contiguous again to the marble isle of Paros in the Archipelago, which has furnished such noble material to Grecian sculpture, we find the dark igneous rocks of Santorin, thrown up by successive eruptions, some of which are historically known to us. Of these several volcanic areas in the Mediterranean we would especially direct attention to the Lipari Islands, as replete with interest both from the number and singularity of their igneous minerals, and from the extraordinary volcanic scenery amidst which these are found. The steam-boat, however, now guides the course of all maritime travel, as the railroad does that by land. The lofty cone of Stromboli, ever projecting forth its smoke or flame, occasionally indeed arrests the voyager's eye. But no regular steamer visits these islands; and they are left unseen save by some stray geologist, who, deserting for a moment his Silurian or Tertiary Strata, comes to the study of these more recent changes which have acted on and disturbed the present crust of the globe.

In close physical relation to its volcanoes are those great Earthquakes by which in all ages the basin of the Mediter-

* We speak here by a willing anticipation of what may yet be done at Herculaneum under the auspices of a better government. If we may hope ever to fill up from papyri any of the lacunæ in the Greek works of antiquity, the buried ruins of this city offer the best chance of success. What has hitherto been obtained from it might seem to belong to the library of some littérateur of the Alexandrian school. Future excavation may perchance afford us (and possibly under better preservation of the papyri rolls) fragments of \#schylus, Sophocles, or Menander, or portions of the lost books of Livy; - treasures of greater value than the writings of any grammarians or sophists. 
ranean, and especially its central and eastern portion, has been shaken and devastated. Omitting the various records of earlier date, we may notice the earthquake in the reign of Valentinian, described by Ammianus Marcellinus, which spread wide calamity over its coasts and isles; destroying, as is affirmed, 50,000 persons at Alexandria alone by the sudden flux and reflux of the sea; - and those yet more frequent and violent in the time of Justinian, described by Procopius and other writers, in one of which the city of Antioch is reported to have lost 230,000 of its inhabitants. All such numbers are of course gross exaggerations, but exaggerations which express the magnitude of the calamities sustained. Under the same qualification we may mention the series of great earthquakes in 1693, of which Sicily seems to have been the centre, with a recorded loss of 80,000 or 90,000 of its population; and the Calabrian earthquakes of 1783 and 1784 , extraordinary from the frequency of the shocks, of which about 1,100 were registered at Montaleone, the seeming centre of these subterranean convulsions. In the very last year (1856), the whole eastern basin of the Mediterranean was shaken by a violent earthquake, from which some thousand persons are alleged to have perished in Candia alone. Certain areas in this sea, and without any close volcanic proximity, are subject to what may be called a chronic form of the phenomenon. Such an area exists among the Ionian Isles; where the year rarely passes without some terremoto greater or less in violence; and where daily shocks are not uncommon for several weeks in succession. Those who have visited Zante and Santa Maura will recollect the many traces of such concussions scattered over these islands. In 1853 a smart shock of earthquake was felt over the mainland of Greece, which overthrew most of the existing habitations of Thebes, and caused great alarm in Athens. This occurrence is the 
more worthy of notice as we do not recollect any earthquake recorded to have taken place in Attica; and the state of the architectural monuments of Athens shows that they have not suffered in former times from this formidable cause of destruction.

The Geology of the Mediterranean basin is a subject which would lead us beyond our present limits. To give a bare outline of it would be, in fact, to enumerate as appearing in different localities of its coasts and isles almost every one in the long series of formations, - from granite and the primitive slate rocks to the newest tertiary strata laden with the shells of existing seas. Though the zealous activity of geologists during the last quarter of a century has left few regions untouched, there are yet certain portions of coast as in Asia Minor and on the southern shore of the Black Sea, - which require, and will repay, a much closer examination. This is especially true as regards the fossiliferous strata, those wonderful exponents of ages and conditions of the earth prior to the existence of man. The localities just named are near to that region which bears the earliest records of the human race; and the research here, (though in no way affecting the physical relations of time and succession already determined,) would have an interest peculiar to itself, in associating these records with the more silent antecedent memorials, stamped in their long series on the strata beneath.

On the zoology and botany of the Mediterranean we are equally unable to dwell, seeing the multitude of details they comprise, and the difficulty of reducing them to any brief delineation. A future work on this Sea, such as we desire to obtain, must attest its completeness by embracing these topics in their full extent, and with all the aids and results of modern research. Such research directed to this locality has certain interests which may well sanction and encourage it. A vast circuit of profound sea, so nearly severed from 
the ocean, is sure according to all analogy to possess a Fauna and Flora more or less peculiar to itself; with subordinate distinctions from the various climates of the lands adjoining it, and the deep penetration of its gulfs into the surrounding continent. But there is a further interest, yet more appropriate to the Mediterranean, in the relation of its living natural history to the more ancient records we possess of animal and vegetable life. History, poetry, and philosophy are alike illustrated by the identification of species; and by the removal of those errors which a vague nomenclature and imperfect classification have propagated from one age to another. This has been strikingly exernplified on various coasts of the Mediterranean of late years, and especially in Palestine and Egypt; where the illustration blends itself with sacred history, and scientific research becomes a living commentary on the books of Scripture. Those who have read Dr. Stanley's admirable work on Syria and Palestine, will appreciate the value as well as interest of the evidence derived from this method of research. Another striking instance of such illustration may be found in the researches of Professor E. Forbes, among the Mollusca and Radiata of the Egean Sea; which, while attesting his own merits as an observer, give proof of the wonderful knowledge and accuracy of Aristotle in this as in so many other departments of natural science.

A volume might be written on the Rivers of the Mediterranean. While forming a remarkable part of its physical history, very many, perhaps the greater number, are familiar to us under the same classical association as the shores whence they issue into the sea. No natural features indeed fix and endear these associations so much as rivers. They mark and define the events of history; they feed with pleasing or pathetic images the fancy of the poet; they furnish even the most profound philosophy with illustration 
and example. From that furthest extremity of this great inland Sea, where

Mæotis sleeps, and hardly flows

The freezing Tanais through a waste of snows,

to its egress in the Atlantic Ocean, we have a succession of streams all more or less known to classical fame. We cannot stop to enumerate them in full. But in naming the Don, the Dnieper, the Danube, the Nile, the Po, the Rhone, and the Ebro, as those of greatest length and volume, we leave to the recollection of our readers those numerous lesser rivers of Asia, Greece, and Italy - the Mæander, Hebrus, Peneus, Alpheus, Eurotas, Tiber, \&c. - which have become almost as household words to our literature and speech. English poetry, perhaps more than any other of modern time, has drawn plenteously from these names and records of ancient streams; thereby satisfying at once those classical feelings and that love of natural beauty, which we believe to be more deeply cherished in England than in any other country.

Of all the rivers which enter the Mediterranean, the Nile is beyond doubt the most wonderful. Scarcely should we err in calling it the most wonderful in the world. The St. Lawrence, from the volume of water which it pours through inland seas and forests, and over cataracts and rapids unrivalled in grandeur, comes closest to it in the comparison. But the latter river is utterly wanting in those marvellous monuments of ancient empire which have hallowed the Nile to all succeeding ages, and which we are still disinterring and deciphering for those who come after us. Its waters, while reflecting these great monuments as they flow through Egypt, give exuberant fertility to a country which would else have been a portion of the adjoining desert. The line to which they reach in their annual flood, abruptly divides a sterile sand from the most profuse and vigorous vegetation; 
and Seneca in no wise exaggerates in saying that to the Nile Egypt owes not merely its fertility of soil, but the soil itself. (Nat. Qucest. lib. iv.) Though somewhat beside our subject, we could willingly descant on various other peculiarities of this majestic river. Such are its singular parallelism to the prolonged gulf of the Red Sea; - its flow of 1,200 miles through Nubia and Egypt, without the addition of a single stream to its waters, which thereby actually decrease in volume as they descend to the sea; - the wonderful persistence and uniformity of that vast periodical flood, which, coming from unknown sources, has, for at least four thousand years, preserved the same times of rise and fall; - the equal steadiness of that old Etesian wind (the Etesia flabra) which meets and stems the descending waters; - and, above all, the mysterious problem, still unsolved, as to the origin and true fountains of this great river. Recent research, approaching within a short distance of the equator, has made it almost certain that they are to be sought for near to, or even south of this line. But whether in mountains of perpetual snow, - or in a lower, but still elevated region of lakes and swamps, fed by the periodical rains of the tropics - is a question yet open to the enterprise of the traveller. Our opinion tends strongly to the latter view. That the discovery will be made within the next few years we hold to be certain, seeing the zeal and new appliances directed towards it. The man who accomplishes it will perpetuate his name to all future time, even though he do but confirm that statement of Ptolemy, to which modern discovery is every day lending fresh weight.

The number and magnitude of the Mediterranean rivers has given rise to some curious enquiries - suggested even as early as the time of Dr. Halley - as to the disposal of the mighty volumes of water thus poured into the sea; and added to by the rains, averaging sixteen or seventeen inches 
annually, which fall upon its area. The level remaining the same, the question occurs how this balance is maintained? It cannot be by efflux into the Atlantic, since the only notable current in the Straits of Gibraltar is a central one, setting constantly inwards from the ocean; at the average rate between Tariffa and Alcagar Point, where the passage is narrowest, of nearly three miles an hour. Halley urged, and made experiments to prove, that simple evaporation from a surface of this extent and in this latitude, was fully equal to explain the phenomenon; and though some of his postulates are doubtful, the general conclusion may fairly be admitted. It is a fractional part and local evidence of that great balance or law of compensation which prevails over the globe; blending together the various elements of force and action into one constant and harmonious result. Whatever seeming disorders may occur in the working of these elements, they so neutralise one another in time and locality as to maintain unimpaired that equilibrium which, on a small scale, is often vainly sought for by human labour and ingenuity.

We must remark, however, that there is still a good deal of uncertainty as to the eastward current in the Straits of Gibraltar. What the deviations are from its average velocity, and how produced, are points not yet ascertained. Nor have we any certain proof as to a point more disputed; viz. whether there be not an under-current, carrying outwards an equivalent to the volume brought in from the ocean. Two or three ambiguous stories of sunken ships, transported westwards, have been accepted as partial proof to this effect. Stronger presumptions have been drawn from the necessity of such outgoing equivalent to the surface current setting in, - from our knowledge of counter-currents of similar kind in other oceans and seas, - and from the fact observed of the increasing specific gravity of the waters of the Mediterranean within the Straits as thëir depth augments. These conjec- 
tures, however, admit of being converted into certainty; and doubtless will be so, with the augmented means which every year affords for such researches.

The common belief that the Mediterranean is a tideless sea is not strictly true. It is too vast a basin not to feel in itself, independently of those ocean tides which are almost lost in the ingress of the Straits, that wonderful influence of the moon and sun, acting by periods of endless intermission on all the great waters of the globe. But nevertheless the surface is not large enough, nor the egress to the Atlantic wide enough, to allow of those successive displacements and replacements of water which are essential to the complete phenomena of tides ; while the winds and the fluctuations of atmospheric pressure, even more marked and frequent here than on the ocean, have far greater effect in disturbing, or annulling altogether, the slight tidal elevation actually attained. It is only under certain local conditions, like those which in the Bay of Fundy, the Bristol Channel, the mouth of the Ganges, \&c., raise the tides to forty or fifty feet, that the waters of the Mediterranean show distinctly this influence. Wherever the line of coast is contracted into a strait, or approximates to the funnel form, there the tide generally becomes obvious. In the Faro of Messina it rises from twelve to twenty inches, and occurs with some regularity. At the northern extremity of the Adriatic, at Venice and Chiozza, the rise often reaches two or three feet; greatly influenced by the winds of the gulf, but not depending on them. In the Gulf of Corinth similar but slighter effects have been observed; and again in the narrow Strait of Negropont, the ancient Euripus, we find a very singular and perplexing alternation of currents, - partly, as it appears, true lunar tides with periodical rise of waters, partly the effect of irregular winds and of the tortuous lines of mountain coast which mark this extraordinary locality. 
The greatest of Greek naturalists - Aristotle - died within actual sight of these tidal phenomena; but we may very safely reject the old story that he drowned himself in the Euripus, because unable satisfactorily to explain them.

The colour, luminosity, and saltness of the Mediterranean are discussed by Admiral Smyth in one of his chapters. The clear blue tint of its waters has been commemorated from early time; and, in the absence of strong tides or currents, it is probable that such colour may be more constant in this deep inland sea than in the ocean at large. But the rule is the same here as in the open ocean:- shallow water is indicated by a green tint of different degrees, profound depth by an indigo blue colour. In that wonderful phenomenon of animal life - the luminousness of its waters the Mediterranean is at least as remarkable as any other sea. Some observers have considered it to be more so; and it is doubtless possible though not proved, that the recesses of an inland sea with few currents, highly saline, and shown by its volcanoes to be closely contiguous to sources of subterranean heat, may favour the generation of these singular forms of animal life, which perplex the imagination equally by their profuse abundance and exquisite minuteness.

Regarding the saltness of this Sea our information is somewhat ambiguous. The most exact notices appear to be those of Borillon la Grange, which give the average proportions of saline matter as fully four per cent.; or about onetwelfth more than that of the ocean. But this may vary in different parts of the Mediterranean; and a further and more curious question relates to the proportion at different depths. In the case of a profound basin with a narrow egress, continually receiving saline matters both from the ocean and land, and losing none by evaporation, - the presumption occurs that the water may become more saline and denser at its greater depths, and may even in places deposit beds 
of salt at the bottom of the sea. No such deposits have been found in sounding; but the observations of Wollaston upon specimens of water obtained from different depths, show a specific gravity greatly augmented at the deeper levels; instanced especially in one case, where water from a depth of 670 fathoms about fifty miles within the Straits, was found to contain four times the usual quantity of salt; and to have a specific gravity equivalent to this large excess. This is so singular a result as to have created doubt respecting its accuracy. Dr. Wollaston's inference from it that there must be a dense lower current running out of the Mediterranean, and carrying into the Atlantic the surplus salt of this inland sea, can hardly be maintained in face of the fact already mentioned of a bar traversing the Straits, with a maximum depth at this place of 150 fathoms. No stream of denser water, coming from greater depths, could rise upwards so as to surmount this impediment. The question altogether must be left to future and more multiplied observations.

The physical history of the Mediterranean cannot be complete. without some notice of the winds, which move this great mass of inland waters. We do not find here, nor could we expect their existence, the constant or strictly periodical atmospheric currents which sweep over the wider oceans of the globe. In a landlocked basin thus irregular in outline, studded with mountain-isles and girt round in great part by mountain chains, local causes modify or predominate over those general conditions to which the atmosphere is subjected by the rotation of the earth, and its annual revolution round the Sun. To other influences on the winds of this Sea must be added that of the vast African desert, stretching for 2,000 miles in a direction parallel to its southern shore, and in parts touching upon it; - an enormous waste of bare sand or rock, vehemently reflecting the rays of a southern sun, and acting as a furnace on the atmosphere above it. In 
effect of these and other circumstances, the winds of the Mediterranean, though to a certain extent regular and periodical, yet abound in local characters and local names; and we might readily enumerate more than a dozen, pertaining to different coasts or gulfs, - as the Birazones of the SE. coast of Spain; the Vent de Bize, or Mistral of the southern French coast; the Raffiche of Corsica and other mountainous islands; the Gregale of Sardinia and Malta; the Siffanto and Bora of the Adriatic; the Tramontana generally over the Levant; and the Levanters and Sirocco of the whole Mediterranean. Of these several winds the Sirocco, or south-easterly wind, is by far the most remarkable; not merely from its frequency and wide prevalence,

- but yet more from its physical properties, and peculiar effects on the animal frame. These effects, mainly manifested on the nervous and muscular power, are now become too familiar to the traveller to need being dwelt upon in detail. Every one who has felt this wind as it occurs at Malta or Palermo, will well remember that prostration both of body and mind, which is its instant and continued effect - an effect certainly not owing to temperature alone, since winds of greater heat may blow from other quarters without producing the like results. Various circumstances make it probable that atmospheric electricity is concerned in these phenomena; but we need minute and prolonged observations, like those of Peltier and Quetelet, to satisfy the demand for facts, before this or any other hypothesis can stand good. Such research might be readily carried on at Malta; conjoined with observations as to the proportion of Ozone, and other properties of this strange and malignant wind, the local relation of which to the African and Arabian deserts, and to the Samiel wind of Egypt, will at once occur in any speculation as to its causes.

The frequent suddenness and violence of Mediterranean 
storms are well known to those who have been voyagers in the Gulf of Lyons and the Archipelago. But we must add a few words also as to the calms of this deep sea - the bonaccia of the Italian mariner - those times when its waters sleep under the sun for days together, as if they had never been ruffled by wind or storm. The voyager in the Mediterranean in older times loitered long and wearily under these calms. The traveller of our own days presses forwards despite them; with the aid of that ever constant motive power, created by and subjected to human skill. Yet even he may well long for breezes to stir the still surface, and

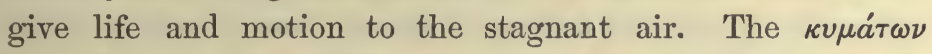

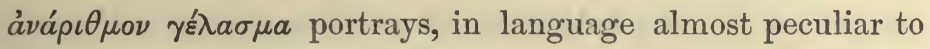
the great poet who uses it, that happier aspect of seas which gladdens with movement the eye of the sailor; - such as Claude so often and so fondly conveys to his canvas, with accompaniments which the Mediterranean shores alone can furnish to the painter.

That strange and still only partially explained phenomenon of the Water-Spout, is a very frequent occurrence in the Mediterranean. While all human things have changed over and over again within its circuit, these wonders of nature, even such as seem most eccentric and anomalous, remain what they ever were. The descriptions of Lucretius and Pliny picture the 'demissa columna de colo' (the $\pi \rho \eta \sigma \tau \eta \dot{\rho}$ of the Greeks), as exactly as the poet or naturalist of our own day would describe it. Were it not foreign to our subject, we would willingly pause here to invite attention to the general theory of these circular or vorticose movements, as they occur in so many phenomena of the natural world; - from the slender spiral of sand, which on a dusty day whirls rapidly before us on the road, to the vast moving columns of the African desert - the water-spout which sucks up the sea into the vacuum within its whirl, - and 
the Cyclone or circular hurricane, which sweeps over the ocean, a calm girt round by a whirlwind. Even earthquakes, in some of their phenomena of propagation and vibration, come under the same analogy; - strange though it may seem that the solid crust of the globe should in any way be submitted to that law of interference of waves of horizontal vibration, which produce a rotatory movement in the case of the circular polarisation of light. Imagination, pressing yet beyond, and looking especially to the composite nature of the motion in all these cases, might extend the relation even to the great movements of the solar system; or still more remotely in space to the spiral forms of nebulæ which Lord Rosse's telescope has disclosed to us. But putting aside these more hypothetical analogies, we have sufficient reason to associate together, under some cause or principle of motion not yet wholly understood, the simpler terrestrial phenomena to which we have just alluded; and many others which come, though less explicitly, under the same law of action. And this is one of the many physical questions still open to research, and inviting both observation and experiment in aid of the solution which it is sure in the end to obtain.

We have lingered so long on the physical history of the Mediterranean, that little room is left for comment on that other part of its history, of which men-and nations form the subject and the interest; - a subject indeed so vast, and an interest so various, that it would be futile to attempt more than a mere outline of what can only be compassed in a complete work. But the topic is closely blended with, and illustrated by the physical history of this Sea; and much may be gained to the clearness and concentration of human. history in its most important epochs, by grouping its events in their relations to these remarkable shores. In each successive age, from the earliest date and record to which we 
can reach, the Mediterranean has been the region upon, or around which, races and nations have struggled for possession and power. Egypt stands first in the long series of sovereignties which border upon it; - reaching remotely into the depths of time in its pictured, written, and monumental history; yet owing its very existence, as well as its wealth and power, to the great River which seeks the sea through this extraordinary valley. The Assyrian, Babylonian, and Persian Empires, though less closely contiguous to the Mediterranean in the centre of their power, yet are deeply concerned in the successive events of war and conquest of which its eastern shores were anciently the scene. The history of the Jewish nation, closely connected with that of the Empires just named, yet having a special and miraculous individuality of its own, belongs by close proximity to this Sea; with which it is associated not only by the record of events, but also by those many sublime passages of sacred poetry, of which the 'wonders of the deep' are the worthy and magnificent theme. Still more closely appertaining to the Mediterranean is that strange and anomalous commonwealth of the Phœnician Cities; anticipating in some unexplained way the progress of later times, and carrying its commerce by sea or land to the extremities of the then known world. The people and commonwealths of Greece next come into the picture; - a wonderful race, deriving some parts of their primitive culture from Egypt and Phœnicia; but so enlarging and refining these elements, as to have bequeathed an immortal legacy of art, poetry, and philosophy to every succeeding age. The Mediterranean, girding round and intersecting every part of their territory, is the constant scene of Grecian history and poetry. No one familiar with Herodotus and Thucydides can forget how large a portion of their narrative is attached to the coasts of this sea; which attest even now in their several localities 
every most striking incident recorded by these great historians. Marathon, Salamis, Thermopylæ, Sphacteria, Syracuse, all afford present and living pictures of the site of events thus consecrated to our memory. Within the same remarkable epoch is comprised that signal event of the Macedonian conquests, begun on the shores of the Mediterranean, carried by an impetuous march of victory to those of the Indian Ocean, and long surviving the great warrior who achieved them.

This earlier portion of history belongs chiefly to the eastern end of the Mediterranean and its subordinate seas. Descending with the course of time, and shifting the scene westwards, we find the Republics of Rome and Carthage struggling long and fiercely for supremacy on its shores; and in their earlier wars, by a series of naval conflicts unparalleled in maritime history. Despite the genius of one great Carthaginian chief, Rome triumphed in the end through the vigour of her civil institutions and consummate military system; and triumphed not over Carthage alone, but eventually over every other Mediterranean power. No fact in all history approaches in wonder to this great miracle of the Roman Empire - the progress from an obscure town on the Tiber to the complete supremacy of the ancient world. During two or three centuries of this Empire, even amidst internal revolutions and disorders, there was no part of the vast circuit of the Mediterranean which did not own the Roman sway. And yet later, when the nominal seat of government was translated to the Bosphorus, and hordes of barbarians - Goths, Huns, and Vandals - pressed upon the empire on every frontier of this wide circumference, the Mediterranean and its rich coasts still formed the centre and object of these great movements of races. And so they continued during the dark and gloomy centuries which separate the ancient from the modern world. 
The great event of these intermediate ages was undoubtedly the vast and sudden growth of the Arabian power. This extraordinary empire, the joint creation of the Koran and sword, though not limited to the shores of the Mediterranean, yet brought into permanent subjection its whole southern coast from Egypt to Morocco, and for some centuries the largest and richest part of Spain; carrying terror meanwhile to all other coasts of this sea, and once to the very gates of Rome itself. The decay of the eastern Caliphate was hastened by the ruder invasion of the Turkish tribes, who, adopting the religion, succeeded to the conquests and power of the Arabian races. The Mediterranean, in its coasts and islands, was still the chief scene of this struggle of races and religions; lasting even beyond the conquest of Constantinople by the Turks, and including the history of those romantic expeditions, which, under the name of Crusades, brought successive armies of warriors and zealots from the west of Europe to the deliverance of the Holy Land. During the 175 years which elapsed between the first preaching of Peter the Hermit and the fatal crusade of St. Louis, the Mediterranean furnished the road to these fierce and fitful conflicts, which the unchristian passions and mutual jealousies of the Crusaders rendered so utterly unavailing in their issue. A striking episode in this portion of history is that furnished by the Norman race; - winning for itself sovereignties in this southern sea by the same impetuous valour which signalised their enterprises in Northern Europe. Though any active struggle against Islamism has ceased during the last three or four centuries, yet the Mediterranean, in its whole extent from the Straits of Gibraltar to the foot of the Caucasus chain, still forms a line of partition between Christian nations and those of the Mohametan faith; and the outrages of Barbary corsairs and pirates were continued even to the close of the great continental war. 
We ourselves have seen, in its very centre, and when the naval power of England was supreme on these waters, the spectacle of an Algerine squadron hovering round Sardinia; capturing coasting vessels and Greek merchant ships; and making descents on the coast to plunder villages and carry off peasants as captives. Even yet later the Isles of the Archipelago have been the resort of less disciplined but fiercer pirates, the wild off-cast of those adjacent countries which are still only half submitted to the Turkish yoke. These things no longer exist, and can never recur; but it is disgraceful to European civilisation that they should have endured so long.

We have no space left us to extend or fill up with details the summary just given of the political history of the Mediterranean; but this enlargement may safely be left to the memory of our readers; - as well of those who have studied the history of the world under the light of a large philosophy, as of others who have followed it through the various localities forming the scene of its events, and often giving motive and direction to their progress. We feel assured that in recommending the whole subject of the Mediterranean to more especial attention, and seeking to associate together its physical and political history for their mutual illustration, we are contributing to forward the scheme of some future work, which may more largely embrace the topics we have here only slightly indicated. The interest of Admiral Smyth's volume cannot be lessened by such undertaking. It will remain - especially if receiving in a future edition the knowledge since gained - the most valuable record we possess of the hydrography of the Mediterranean; and will furnish important materials to the more complete and classical work we are solicitous to suggest. 


\title{
METEORS，AEROLITES，SHOOTING-STARS.*
}

\author{
[Quarteriy Review, No. 183.]
}

$\mathrm{N}$ every age of the world, and in every region of it, there
have been witnessed, amidst the more constant aspects and phenomena of the heavens, those strangely irregular and vagrant lights, those 'fiery shapes and burning cressets,' which suddenly kindle into brightness above us, and as suddenly are lost again in darkness. Sometimes seen as globes of light in rapid movement - much more frequently under the aspect and name of falling or shooting stars, and these occasionally even crowding certain parts of the sky by their number - such appearances in former times were regarded either with dull amazement, or with superstitious awe as the omens of approaching events. Throughout all ages, moreover, reports have existed of masses of stone of various size falling from the sky, preceded by vivid light and explosion; and these occurrences, as might be supposed, have in all former times and by every people, been similarly made the subject of superstitious belief. The Ancyle or sacred shield of Numa, the holy Kaaba of Mecca, the sword of the Mongolian Emperor, and the great stone of the pyramid at

* 1. Recherches sur les Étoiles Filantes. Par MM. Coulvier-Gravier et Saigey. Introduction Historique. Paris, 1847.

2. Catalogue of Observations of Luminous Meteors. By the Rev. Baden Powell, M.A., F.R.S., Savilian Professor of Geometry, Oxford. In Reports of British Association, for 1847, 1848, 1849, 1850, 1851.

3. Humboldt's Cosmos. Translated under the superintendence of Lieut.-Col. Sabine. Vol. I. Section on Aerolites. 
Cholula in Mexico, have all the same history annexed to them. They fell from heaven, and were venerated in the divine origin thus presumed. These falling stones, however, though more wonderful in many respects, were much less frequent than the meteoric lights which blazed before the eyes of nations; and their fall was for the most part very vaguely recorded. As we shall see afterwards, it is only within the last sixty years that science has fully admitted them within her pale; - reluctantly, it may almost be said, as well as tardily; and resting even more on proofs furnished by the physical characters of the bodies themselves, than on the historical evidence of their descent.

Nevertheless, it is chiefly to the recognition of these Aerolites or falling stones, that we owe the zealous scientific research which has since been given to the subject of Meteors at large. However wonderful these phenomena might be in themselves, their aspects and periods were seemingly so irregular as to render them insusceptible of that classification of facts which is the basis of all true science. The untutored gaze of the multitude was for ages as productive of results as the observation of the naturalist; and until very recently the theories of the latter scarcely went beyond certain vague notions of inflammable gases or electrical actions in the atmosphere. The bog-vapour kindled above the earth instead of on its surface - and yet more the phenomenon of lightning under its various aspects - offered explanations just plausible enough to check all further investigation; and when Dr. Franklin (little more than a hundred years ago) first drew electrical sparks from a thunder-cloud, it seemed as if a sufficient cause for meteoric appearances had been fully obtained. Yet, though the dominion of this great element of Electricity has been extending itself to our knowledge ever since, we shall presently see that other causes are here 
concerned; and that we must carry our speculations still higher, before we can compass all the facts which modern observation has placed before us.

It will be readily conceived how much the admission of the fact, that Meteors are sometimes accompanied by the precipitation of stones or earthy and metallic matters from the sky, affected every part of this enquiry. And when Chemistry intervened, disclosing the singular and very similar composition of the bodies thus strangely conveyed to us, it became obvious that new elements were concerned, of which science was required to take larger cognizance. About the same period, research was more exactly applied to determine the height, velocity, and direction of meteors, and especially of falling stars, while luminous to the eye. The results of this enquiry, though embarrassed by various difficulties, tended yet further to remove their physical causes beyond the region of our globe; by showing their elevation above the atmosphere, their vast rapidity of passage through space, and lines of movement involving other forces than that of simple gravitation towards the earth. And when to such researches were added, more recently, certain remarkable facts as to the periodicity of falling stars, the enquiry assumed at once a cosmical character, associating itself with some of the movements and higher laws of the planetary system.

We have sketched this preliminary outline of the subject, from a feeling of the interest which ever attaches to the successive stages of a new science; - those steps by which we ascend from the rude, doubtful, or superstitious record of isolated facts, to the absolute proof, the classification of phenomena, and the determination of the physical laws which govern them. Such notices are not more instructive as to the philosophy of the material world than in relation to the history of man himself, thus advancing in knowledge and power amidst the elements which surround him. 
Though the subject of Meteors was now brought within the domain of science, the difficulty remained of giving any classification to the phenomena, on which to base enquiry into their causes and physical connections. On what principle was it possible to arrange appearances so vague and various in time, place, magnitude, and brilliancy? Even now the simplest division is perhaps the only one admissible; expressing little more than those external aspects to which we have already alluded, without reference to the physical causes which are doubtless concerned in their varieties. First in order we have the globes or balls of light (bolides), appearing suddenly, often loudly exploding, and having certain physical characters to which we shall afterwards advert. Secondly, falling or shooting-stars (étoiles filantes), seen at all times and in all countries, but more numerously at certain periods, and more frequently under the clear skies of tropical regions. Thirdly, Aerolites or meteoric stones, differing greatly in size and form, but with various characters showing some common cause or origin, and this wholly alien to the planet on which they fall.

The spirit of enquiry awakened on the subject of Meteors, and its objects thus far defined, it was natural to recur to history and tradition for evidences of similar phenomena in prior ages. This research, as we have already intimated, was fertile of curious results - derived as well from the classical writers of Greece and Rome, as from the records of the dark ages and of every intervening century to our own time. The most remote regions, as well as periods, contributed to this testimony; - the facts sometimes coloured by superstition, sometimes obscured by imperfect report; but numerous and exact enough for comparison with our own observations, and giving full proof of the identity of the phenomena throughout. Poetry naturally busied itself with these vagrant lights of heaven; and we might cite various passages from the 
Greek and Latin poets (Lucretius very especially), which, though in some part ambiguous from the association of lightning with meteoric appearances, yet manifestly include the latter in their description.* The historians of antiquity denote them in more or less detail, and with various degrees of belief. The naturalists of Greece and Rome, from Aristotle down to Seneca and Pliny, have not only left descriptions copious enough to identify all the appearances with those of our own time, but have here and there offered suggestions as to natural causes which are fairly admissible among the hypotheses of more recent date.

But the highest interest in these records of past times attaches itself to the fall of Aerolites; and as we propose to take this class of meteors first into view, we may reasonably dwell for a moment upon their early history. The phrases of Lapidibus pluit - Crebri ceciderunt a colo lapides, \&c., are familiar to us from Livy, and may no longer be disregarded as the idle tales of a superstitious age. Aschylus, in the fragment we possess of his Prometheus Unbound, alludes to a shower of rounded stones sent down by Jupiter from a cloud. But the most remarkable and authentic record of antiquity is that of the massive stone which fell in the 78th Olympiad (about the time of the birth of Socrates) at Egospotamos on the Hellespont; - the place soon afterwards dignified or defaced, as opinion may be, by that naval victory of Lysander which subjected Athens and Greece for a time to the Spartan power. The philosopher Anaxagoras was said to have predicted the fall of this stone from the sun;- a

* Virgil, in the more practical description of his Georgics, connects falling stars with the approach of wind-

Sæpe etiam stellas, vento impendente, videbis

Præcipites cœlo labi, \&c.

Both Theophrastus and Pliny admit the same idea. If this connection were generally true, which we doubt, it probably depends merely on the rising wind dispelling vapours which before hid these meteors from sight. 
prediction, like many others, doubtless following after the event. It is expressly mentioned by Aristotle; by the author of the Parian Chronicle; by Diogenes of Apollonia, who speaks of it as 'falling in flames ;' and most fully by Plutarch and Pliny, both of whom distinctly state it to be shown in their time - that is, in the sixth century after its fall. Pliny's description is well marked - Qui lapis etiam nunc ostenditur, magnitudine vehis, colore adusto ; and he adds the fact that a burning comet accompanied its descent.*

We see no cause whatever to doubt the authenticity of this statement, of which the very phrase colore adusto is a striking verification. If the mass remained visible down to Pliny's time, and of such magnitude as described, it is far from impossible that it may even now be re-discovered; - with the aid perchance of some stray tradition attached to the place, surviving as so often happens, the lapse of ages, the changes of human dominion, and even the change of race itself on the spot. Only one slight effort, as far as we know, has been made for the recovery of this ancient aerolite. We wonder that some of our many Oriental travellers do not abstract a few days from the seraglios, mosques, and bazaars of Constantinople - (and, we fear, we must further add, from the lounging life of the Pera hotels) - to engage deliberately in the attempt. Fame earned by discovery in travel is no longer so common a commodity that the chances of it should be

* Plutarch, who reasons with force and pertinency as to the origin of this stone (in Vitâ Lysandri), explicitly states that it was still held in much veneration by the inhabitants of the Chersonesus. He also speaks of its vast size, and of the tradition of a fiery cloud or globe which preceded its fall. In his

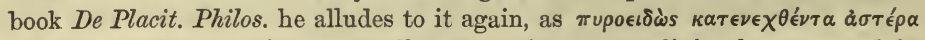

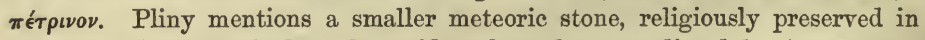
the gymnasium at Abydos, also said to have been predicted by Anaxagoras. This coincidence of time and place might lead to the suspicion that both were derived from the same meteor. He further notices a stone of recent fall which he had himself seen at Vocontii in the province of Gallia Narbonensis - now Vaison in Provence. 
disdained. In this case the research, if successful, would be of interest enough for both history and science to perpetuate the name of the discoverer.*

While the writers of Greece and Rome, as well as of the middle ages of Europe, furnish us only with scattered notices of these aerolites, it is far otherwise with the Chinese; - that singular people, whose language, institutions, and methods of thought might almost suggest them as a race of men struck off from some other planet. There exist in China authentic catalogues of the remarkable meteors of all classes, aerolites included, which have appeared there during a period of 2,400 years. To give an idea of the minuteness of these records (the translation of which we owe to the lamented $\mathrm{Ed}$. Biot) it is enough to mention that in the three centuries from A.D. 960 to 1270 not fewer than 1,479 meteors are registered by the Chinese observers, who seem to have

* Though the locality of this stone is not further indicated than by the statement of its fall at Egospotamos, yet the invariable manner in which it is thus described defines tolerably well the district to be examined. We learn from the old geographers that there was a town called Egospotami on the shores of the Hellespont, and we may infer a stream or streams, from which its name was derived. The description of the naval fight and the situation relatively to Lampsacus (the modern Lamsaki) further define the locality within certain limits. The traveller devoting himself to the research might make his headquarters at various places near to the spot in question. He should render himself previously familiar with the aspect of meteoric stones, as now seen in every Museum and Mineralogical collection. He should study the character of the rocks and fragmentary masses in the vicinity, so as more readily to appreciate the differences of aspect. He must expect the possibility of a small part only of the mass appearing above the surface; and his eye must be awake and active for any such partial appearances. If the stone sought for were wholly concealed by alluvial deposits, the research would be vain, unless happily aided by some local traditions, as we have noticed above.

Had not my own voyages been necessarily rapid, and the time of year unfavourable, I should have taken some shame to myself for having three times passed the Dardanelles without halting on this spot. The autumnal months must be aroided, as the malaria fever is always rife at this season on the shores of the strait.

We can hardly hope to recorer any remnant of the great stone which was seen to fall at Narni, A.D. 921, and is described as projecting four feet above the water of the river into which it fell. 
been officially employed for this purpose.* It is only of late years that the science of Europe has placed itself in competition with these extraordinary documents. Though instances of falling stones were continually multiplying themselves in France, England, Germany, Italy and elsewhere, the only memoirs we know on the subject before the time of Chladni, are that of the Jesuit Domenico Troili, and another we shall afterwards notice. The work of Chladni in 1794 formed an epoch in the study of meteorites. This philosopher, still better known by his admirable mode of demonstrating the vibrations and quiescent lines which enter into the phenomena of sound, was the first to collect all the authentic instances of aerolites: a catalogue much enlarged since, but very valuable at the time and showing great zeal of research. Until this moment scarcely one man of science had given assent to the fact, or even considered it as a subject of evidence. The speculations of Kepler, Halley, Maskelyne and others, as to meteoric matters in the planetary space, scarcely touched upon the history or theory of meteoric stones. Yet it would seem a case where history had some claim to credit, since the facts were of a nature which imagination or fear could hardly mystify or distort. Meteors seen and heard to explode - stones at the same time falling to the earth, and frequently discovered and examined at the time of their fall - sometimes falling as single and heated masses, sometimes numerous enough to be described as a shower-in more than one case said to have caused death by stroke of their fragments; - these are things so simple and distinct in narrative that we could not easily refuse belief to them, even had we

* The observations from the seventh century before Christ to 960 were derived by $\mathrm{M}$. Biot from the work of Ma-touan-lin, an eminent Chinese author towards the end of the thirteenth century. Those of the three centuries succeeding A.D. 960 come under the annals of the dynasty of Soung, which during this period had dominion in China. 
less testimony from similar occurrences in our own time. It is one of the many instances furnished by science, of ancient truths, long obscured or discredited, coming suddenly into fresh light and receiving illustration from new and unexpected sources. The chemist's crucible, and the eye of the mineralogist, disclosed results as to these stones which no conjecture could have predicted; and in the end compelled. the belief so long and obstinately denied.

The stone which fell at Wold Cottage in Yorkshire, in 1795, was that which contributed most explicitly to this conversion. Its fall was seen by two persons, following an explosion in the air. It had penetrated to a depth of 18 inches in the soil and chalk whence it was taken. It weighed about $56 \mathrm{lbs}$. Happily it was placed in the hands of an able chemist of the time, Mr. Howard, whose analysis of it was published in the Ph. Transactions for 1802. Yet when Pictet, who had just come from England, read a communication to the French Institute on this subject, 'il y trouva une incrédulité telle qu'il lui fallut une sorte de courage pour achever sa lecture.' A month after, however, Vauquelin produced to the Institute an analysis of his own, fully confirming that of Howard; - a few months later the great fall of stones $(2,000$ or 3,000 in number, ' une véritable pluie de pierres météoriques,') occurred at L'Aigle, in Normandy;-information was obtained about the same time of a numerous shower of stones at Benares, on the Ganges; and similar evidences were multiplied on every side. The fall at L'Aigle, however, may be noted above all, as it led to a local investigation by Biot; who hastened himself to the spot, and with characteristic zeal and ability not merely authenticated the event, but obtained proof as to various incidents attending it, of great value to the true theory of all such falling bodies. Of these the most important was the fact, well ascertained, that the direction of the meteors from which the stones fell must have been 
oblique to the horizon.* The convictions of a man like Biot, founded on personal investigation, may be fairly admitted as another epoch in the history of aerolites.

The striking concurrence of such instances with those of more ancient tradition overcame all remaining doubt; and when Chladni published his second and more valuable work in 1819, with a copious record of aerolites, registered according to the periods and places of their fall as well as the direction of their lines of descent, his statements were received with entire assent by the scientific world. $\uparrow$ His details had the effect not only of authenticating the fall of such stones from the sky, but further, of assigning a meteoric character to certain strange ferruginous masses found in different countries, regarding which only vague traditions existed, or which had no history at all but that of their outward aspect. These masses, some of them of vast weight and dimensions, and manifestly foreign to the localities in which they are found, have enough of kindred with aerolites to justify the name of meteoric iron, and to make it probable that they are of eommon origin. The largest yet known is one estimated to weigh about 14,000 lbs._- discovered at Otumpa, in Brazil, in a locality where there is no iron, nor rock of any kind near the surface. Another, little inferior in size, has been found near Bahia. A smaller mass, but nearer to us, is that from the

* This was ingeniously determined by observing the outline of the surface upon which the fall occurred - found to be elliptical, and not circular, as it would have been had they dropped vertically. The meteor was circular, large, and brilliant - and explosions were heard over a wide tract of country. The stones were hot, and exhaled a strong sulphurous smell.

$\uparrow$ The total number of aerolites which Chladni has registered from the commencement of the Christian era to 1818 is 165 , but some of these must be regarded as doubtful. The distribution of them by countries is ehiefly of value as showing, what might have been expected, the universality of the phenomena orer the earth. From 1600 to 1818 we have the record of 17 in Great Britain, 15 in Franee, 17 in Germany. As to the hours of falling, a large proportion are registered as having fallen during the day; but this difference is readily accounted for, and does not justify an inference as to inequality in the event. 
neighbourhood of Andernach, weighing 3,300 lbs. The volcanic locality might render the origin of this ambiguous; but its analysis by Professor Bischoff of Bonn, in showing a composition of soft metallic iron with a small proportion of nickel, leaves little doubt of its belonging to the class of meteoric bodies. Another remarkable specimen is the Siberian stone, described by Pallas, and which we have ourselves seen in the Imperial Museum at St. Petersburgh, composed of soft spongy iron and olivine. The Tartars on the spot had a tradition of the fall of this stone from the sky, as the Mongolians have of a fragment of black rock, 40 feet high, near the sources of the Yellow River. Other great masses have been found in Australia, proving (if proof were needful) the universality of the phenomenon over the globe.

Before proceeding to the theory of the bodies thus admitted to have been cast upon the earth, we must say something more of their chemical composition - inasmuch as this is not only remarkable in itself, but closely concerned in their theory, and with other speculations of high interest. Collecting the results of all the best analyses down to the present time, we find the actual number of recognised elements discovered in aerolites to be nineteen or twenty - that is, about one third of the whole number of elementary substances (or what we are yet forced to regard.as such) discovered on the earth. Further, all these aerolitic elements actually exist in our planet, though never under the same combinations. No new substance has hitherto come to us from without; and the most abundant of our terrestrial metals, Iron, is that which is largely predominant in all aerolites; forming sometimes, as in certain of the instances just mentioned, upwards of 90 parts in 100 of the mass. Seven other metals copper, tin, nickel, cobalt, chrome, manganese, and molybdena - enter variously into the composition of these stones. 
Cobalt and nickel are the most invariably present; but the proportion of all is trifling compared with that of iron. Further, there have been found in different aerolites six alkalies and earths; namely, - soda, potash, magnesia, lime, silica, and alumina; and in addition to these, carbon, sulphur, phosphorus, and hydrogen. Finally, oxygen must also be named as a general constituent of aerolites, entering, as it does, into the composition of several of the substances just mentioned.

As respects the manner of conjunction of these elements, it is exceedingly various in different aerolites. A few there are, especially examined by Berzelius and Rose, containing olivine, augite, hornblende, and other earthy minerals; and closely resembling certain crystalline compounds, which we find on the surface of the earth. But in much the larger proportion, as we have'said, Iron is the ruling ingredient; and we are justified in concluding that this metal, so remarkable an element in the composition of our globe, exists yet more abundantly in those parts of space, or those aggregations of matter, whence such stones are projected upon the earth.

We need not expatiate on the value of these results. Curious and unexpected in themselves, they will be found, as we pursue our enquiry into the origin of aerolites, to possess a still higher interest as the exponents of conditions of matter extraneous to our own globe. We shall revert to them afterwards in this sense; expressing, meanwhile, our hope that these analyses will be sedulously multiplied as occasions may occur, so as to obtain some larger and more exact generalisation of facts, or perchance the discovery of some element hitherto unknown to us. The same age which has created a circuit for human language and intelligence through wires, water, and earth; and has made the sunbeam execute in a few seconds the most delicate delineations of man and nature, may well aspire to carry its Chemistry into space, 
and to seek conclusions as to other matter than that which surrounds us on the surface of the earth. We may justly apply to the science of our own day a sentence of older date and other application - Si computes annos, exiguum tempus; - si vices rerum, cevum putes.*

We have yet to notice briefly other physical characters belonging to these singular bodies. An important fact is their general fragmentary aspect, as if struck off or detached

* Since this article was first published, the opinion here expressed has been unexpectedly justified by the discoveries made through the method of the Spectrum Analysis. This new and wonderful instrument of research, of which we have elsewhere spoken (page 30 ), in disclosing to us the existence of six known metals in the luminous atmosphere surrounding the Sun, has confirmed the inference drawn from the composition of aerolites, and furnished to physical science a mode of material access to the worlds in space around us. The same method of enquiry (certain, as far as we can yet see, in the evidence it affords) is sure to augment hereafter the knowledge thus obtained. The dark lines of the spectrum left for interpretation are still exceeding numerous, and form in themselves a large mine for research. To the two new metals, Cæsium and Rubidium, first discovered by Bunsen through their spectrum lines, a third (Thallium) has recently been added by the labours of Mr. Crookes; nor is there any obvious limit to the extent to which such discovery may be carried in future.

This new path, however, though a very seductive one, needs much patience as well as zeal to follow it aright. Many perplexing questions occur in the outset, as to the conditions of temperature, vaporous state, chemical action, \&c., which modify or change the refrangibilities of different elements (whether metallic vapours or simple elements) expressed by these spectrum lines. The experiments of Professor Plücker (see Proceedings of Royal Society, 1859), with an apparatus admirably contrived to concentrate the heat of the electric current, show the wonderful influence of heat, simply considered, in determining all these results, and the importance of employing it in its simplest as well as most energetic form. The spectrum lines he obtained for hydrogen, sulphur, \&c., attest the value of this method of experiment, which will doubtless be carried into further use.

Meanwhile, we may repeat the main fact, that we have evidence of several kinds of matter, familiarly known on our own globe, existing also in that greater globe, which forms the centre of our system; - Iron especially conspicuous among these substances. It is needless to speak of the many inferences which hang, almost inseparably, on this single fact. It will at once be seen how much they strengthen our views as to the origins of the remarkable bodies which form the subject of this article; while in more remote speculation, they promise some future knowledge of the matter of other planets, and even of certain fixed stars, which exhibit analogous lines in their spectra, permanently the same for each. 
from some larger mass. Their specific gravity varies greatly according to the proportion of metallic constituents, ranging from twice even to six or seven times the weight of water. The mean is considerably above that of the mineral masses on the surface of the earth, though much below 5.5, or the mean of the whole earth. A notable and very uniform character of aerolites is the shining dark crust enveloping them. It is generally very thin; but indicates by its aspect, and by its entire separation from the mass within, some rapid action of heat, which has not had time to penetrate more deeply into the substance of the stone. These appearances nay give useful guidance to any local search for aerolites of ancient record.

The question as to the mean velocity of aerolites in ap. proaching the earth, can only be settled by approximation, and that perhaps not a very close one. The observations bearing on this point are limited in great degree to the meteoric appearances preceding the fall. The conclusions obtained by Olbers and others would justify the belief in a mean velocity exceeding twenty miles in a second; - a rate of movement further attested by the depth to which many of them penetrate into the earth; and becoming, as we shall presently see, an important element towards the solution of many questions in the theory of these bodies.

The main facts as to Aerolites thus authenticated, the question as to their origin comes yet more forcibly into view. And, in truth, there are few questions more curious - not less to the unenlightened than to men of science - in the novelty and vastness of the suggestions they press upon the mind. Whence, and by what force, do these stones - some of them so massive, all so remarkable in composition descend upon the earth?

It could scarcely perhaps be surmised that five different solutions have been offered in answer to this question. We might even name six, could we for a moment admit the 
notion that these aerolites may be the product of our own volcanoes; - stones forcibly ejected thence, partaking for a time of the motion of the earth, but in the end returning to it. The negative evidence here is so obvious and complete, that we have no need to do more than slightly refer to it. This opinion has no longer an advocate.

A second hypothesis, involving telluric origin, has little more of proof or probability to recommend it. This is, that stones do not actually fall, but that lightning or electricity in some meteoric shape, impinging upon the earth, fuses the earthy and metallic materials on the spot so as to admit of their re-consolidation in these new forms. Other refutation of this opinion is not needed than a simple regard to the composition of aerolites, to their occasional magnitude, and to the great number often appearing at the same time. But, in truth, the notion is one that was never more than vaguely held, and has long since been given up as untenable.

Another solution still has been proposed, also deriving the phenomena from terrestrial causes. This is the hypothesis of atmospheric origin; adopted by many in the outset of the enquiry, from the seeming difficulty of carrying speculation beyond the limits of our globe. Using the fact just ascertained of the identity of the materials of aerolites with elements existing on the earth, they assumed (but without explaining the manner or course of such operation) that these elements might be slowly absorbed into the atmosphere; and retained there in a state of extreme diffusion, until some accidental agency (either electrical or force of other kind) caused their sudden aggregation, and precipitated them on the surface of the earth under the forms and conditions actually observed. In this theory the light, heat, and detonation attending their fall, were attributed to the vehemence of the forces and actions bringing these substances into a solid form from their highly diffused or gaseous state. The 
opinion derived its chief authority from Dr. Izarn's Lithologie Atmosphérique; a book of merit as an historical record, but largely imaginative in all that relates to these metallic and earthy vapours - ' massées sphériquement, et isolées les unes des autres ' - which he presumed to exist in the atmosphere around us.

We speak of this theory in the past tense, because, though at first taken up by many, it was impossible long to maintain it in the absence of all proof, and in the face of facts which gave it every character of physical impossibility. Vauquelin, to whom Izarn addressed his views, explicitly repelled them : - J'aime encore mieux croire que ces pierres viennent de la lune, que d'admettre que les substances les plus fixes que nous connaissons se trouvent en assez grande quantité dans l'atmosphère pour y produire des concrétions aussi considérables que celles qu'on dit en être tombées.' We hardly indeed need comment on the infinite improbability that such materials as iron, nickel, silex, magnesia, \&c., should be absorbed into and exist in the atmosphere; - exist, too, in its upper and lighter stratum, since the most refined analysis has detected no such elements in the lower. Not less improbable is it that matters diffused with such exquisite minuteness, as these hypothetically must be, should thus suddenly coalesce into a dense solid. The action of centripetal aggregation must be carried on simultaneously over a vast extent of space to produce such effect; nor, in truth, do we yet know any physical force or law capable of the peculiar action required. A more positive objection to the atmospheric theory is the direction of movement and fall, as repeatedly ascertained in the case of these bodies. Had they been formed in the atmosphere, whatever the process of aggregation, their fall must have been perpendicular to the earth's surface at the place, instead of oblique, as we generally find it to be. 
Thus compelled to seek for a source beyond the limits of terrestrial action, the hypothesis of lunar origin next came into notice, and was discussed or advocated by philosophers of much higher eminence. Wonder has been called the mother of Wisdom; and bare conjecture has oftentimes long anteceded the researches and results of more exact science. A fall of stones at Milan, about the year 1660, by which a Franciscan monk was killed - (one of three or four recorded instances of death from this cause) - led a naturalist of that country, Paolo Terzago, to publish his conjecture that these stones might come from the Moon. Another great fall of aerolites at Sienna, 134 years afterwards, brought the higher genius of Olbers to researches founded on the same idea, which seems to have been dormant in the interval. In 1795 he examined the question of the initial velocity required to project a body from the surface of the Moon so that it might reach the earth, and determined this to be about 8,000 feet in a second. The lunar theory, and the dynamic questions connected with it, (which Humboldt whimsically entitles the ballistiches Problem, ) speedily engaged the attention of other philosophers. A characteristically bold and terse speech of Laplace, at the Institute, in December 1802, yave impulse as well as sanction to the enquiry. It was made on the occasion already alluded to, when the report of the analysis of meteoric stones by Howard and Vauquelin, and the inferences thence derived, still found an incredulous audience in this learned body.

To that of Laplace may be added the other eminent names of Poissón, Biot, and Berzelius, as successively engaged with the hypothesis of lunar origin; and their respective calculations of the projectile force required were sufficiently alike to justify the conclusion of Olbers, stated above. The argument then stood, and still stands, thus. It is well known that the hemisphere of the Moon, permanently opposed to the earth, 
offers the aspect of mountains of great height, and of numerous craters; - the latter resembling very exactly in character those of our own volcanoes, but much more spacious and profound.* That internal forces exist, or have existed, within this satellite, capable of powerfully disrupting, elevating, and projecting from its surface, must be deemed certain in fact, notwithstanding that all astronomical observation goes to disprove the existence of a lunar atmosphere or lunar seas. Why not suppose stones to be projected thence (no atmospheric pressure existing to retard or arrest them) with force enough to depass the limits of the Moon's attraction, and to come within that of the earth? The calculations just referred to concur in the result, that an initial velocity five or six times as great as that of a ball issuing from the cannon's mouth, might carry a stone so far that it would not return to the Moon; but either continue to revolve subordinately to new attractions, or be precipitated upon a body of more powerful attraction if approaching its sphere. Berzelius went farther in his adoption of the lunar hypothesis ; and, looking to the chemical composition of aerolites, ingeniously conjectured that an excess of iron on one side of the Moon might fairly account for the fact of this side being constantly opposed to the magnetic globe of the earth. .

* The great works of Schrötter, and Beer and Mädler, on the Moon, are well known to our astronomical readers. Not equally known are the curious researches of Mr. Nasmyth, of Manehester, on a certain definite portion of the Moon's surface, about as large as Ireland, named in lunar topography Morolychus. Several years of constant observation given to that one region (a limitation of object generally fertile of results) have enabled this diligent observer to construct a model and maps on a large scale, well illustrating the volcanie character of the Moon's surface, and the vast changes by disruption and elevation which have occurred there.

Mr. De la Rue's admirable application of Photography to the mapping of the Moon, under appliances which only his skill could suggest, furnishes a new mode of astronomical observation, already brought into other use in the phenomenon of solar eclipses, and capable of further extension. 
The hypothesis, thus powerfully advocated, has been displaced not so much by any recent negative proofs, as by the want of further and more assured evidence; and by the adoption of new theories which connect the phenomena of aerolites more directly with those of other meteors, and associate the whole with the general conditions of the planetary system. The lunar theory, to say the least of it, has remained stationary at the point whence it started; nor is there, as far as we can see, any source of fresh knowledge within our reach. Even with the powerful telescopes we now possess, no proof has been obtained of present volcanic activity in the Moon; and, looking backwards to that which may have existed heretofore, we must admit the need of a projectile force much greater than that first presumed, to explain the actual mean velocity of aerolites in approaching the earth. It has been calculated by Olbers (and we believe not disputed) that the initial velocity at the Moon, to satisfy this condition, must be twelve or fourteen times greater than that assigned by Laplace and others; - a projectile force far exceeding that of our own volcanoes, and which, did it really exist, would not cast these masses upon the earth, but cause them, as Olbers and Bessel have shown, to revolve in orbits about the Sun.

Another hypothesis, having kindred with the one just considered, is that which supposes these aerolites to be smaller fragments of that presumed ancient planet between Mars and Jupiter, the disruption of which has produced the many small planets or asteroids, whose excentric orbits cross and crowd each other in this part of the heavens. But a few years ago only four of such ultra-zodiacal bodies were known to us. The position and peculiar orbits of these justified Olbers in his bold conjecture of their fragmentary nature; an opinion greatly strengthened by the later discovery of numerous others in the same interplanetary space, many of 
which we owe to the admirable observations of Mr. Hinde, working with his telescopes in the Regent's Park, almost in the midst of our foggy and smoky metropolis.* These bodies are very various in size; - some of them so small as to defy exact admeasurement. Astronomical considerations fully sanction the idea of a common origin; and if they be truly fragments of a larger body, we may reasonably infer that the same disruptive force which separated them must have projected into space numerous fragments yet smaller, and with orbits more highly inclined to that of the primitive planet in proportion to their smallness. It is another question, however, whether any of these orbits could be such as to bring them in proximity to, and within attraction of the earth. It will be seen that this is simply-a question of possibility, to which little or nothing can be added, or hoped for, in the way of evidence. Like the lunar hypothesis, it remains a mere speculation; affected chiefly by the proofs which have given stronger presumption to another theory.

It is this theory of which we have now to speak; - the one which eonnects meteoric stones with meteors of other kinds; and, recognising in all an origin alien to and beyond the limits of the earth, finds this origin in the interplanetary spaces which were heretofore regarded as void in nature ; - or, if not void, occupied by an imponderable ether, hardly known to us but as a name. Many circumstances have tended gradually to create new views on this subject, and especially the discovery of the vast number of cometary bodies traversing these spaces in all directions;- varying infinitely in magnitude, orbits, and periods of revolution;undergoing great changes even while within our view;-

* The number has rapidly augmented by discovery since this article was written. Nearly seventy now stand in our catalogues (1862), and the heathen mythology has been invoked in vain to furnish names for this planetoid throng. 
some of them seemingly lost, and the orbits of others altered by their approach to the greater planets; one or two of short periods of revolution, and affording proof, by the successive abridgement of these periods, of a resisting medium through which they are moving in their orbits. While contemplating space as thus occupied by so many forms of matter, in such various degrees of concentration, yet all in constant motion, we cannot but suppose that portions of matter still smaller or more attenuated may be in movement around us; apparent only when they come into such contiguity to the earth as to be deflected, or rendered luminous, by its influence. Meteoric stones, we have already seen, are proved to come from beyond the limits of our atmosphere and to enter it with vast velocity. Numerous and exact observations have proved the same to be equally true in the case of shooting-stars and meteoric globes of light. Here then we have a bond of conmection, associating these phenomena under certain common physical forces; while yet leaving ample room for those causes of diversity on which depend the aspects of the different classes of meteors, as well as the individual character of each. Matter in one form or other, variously revolving in the space through which our own globe is moving, is the element with which our enquiry has to occupy itself.

We refer here to the movement of the earth, as well as of these fragmentary or nebulous matters, because both must be supposed concerned in the results. Perhaps some of our readers, even though not wholly unfamiliar with these subjects, may acquiesce in our reminding them, that the globe on which we dwell is at every moment submitted to three separate but simultaneous motions; - of rotation round its axis - revolution round the Sun - and lastly, that vast and mysterious movement by which it. is carried, with the Sun and entire planetary system, through unknown regions of space; - whether as the portion of an orbit round some remote 
centre of attraction, ages may yet be required to show. The grandeur which belongs to such combinations of force, space, and time, cannot be expressed by mere words, and can scarcely be appreciated by numbers. It needs a particular faculty to follow with full comprehension these greater phenomena of the universe; and especially those of sidereal astronomy, to which belongs the translation of the solar system just noticed. It is the peculiar glory of astronomical science in our own time-(the glory of such men as Herschel, Bessel, Struve, and Argelander) - to have determined proper motions in those great luminaries which bear the name of fixed stars; - to have assigned orbits and periods of revolution to numerous double stars; - to have obtained the parallax and measured the distance of many; - to have determined not only the proper motion of our own Sun but also its direction and rate of translation in space. Few can fully understand all that is required in such researches; - the time and intense watchfulness; the exquisite delicacy of instrumental observation; and yet more the genius and mathematical power which can elicit certainty from arnidst the conflicting conditions seeming to render it impossible.

Tempted by the subject to this short digression, we now recur to the argument before us, in which we may presume the second motion of the earth - that of revolution about the Sun - to be chiefly concerned. When we consider this orbit to be so vast that we are, on the 1st of July, distant nearly 190 millions of miles from the place we occupied on the 1st of January, returning again to the same point six months afterwards, we obtain some conception, though a faint one in reality, of the spaces passed through in this great annual motion. If then there be other portions of matter - whencesoever derived, and however fragmentary or attenuated in form and kind - revolving round the Sun, (and we cannot suppose any matter to be stationary in space) 
- it is easy to conceive that the progressive motion of the earth may bring it into such proximity to the numerous and excentric orbits of these meteorites or asteroids, that they become submitted to its influence, and deflected more or less from their course, as we know comets to be by the vicinity of planets; - some actually impinging upon the earth in the form already described; - others merely luminous through certain ares of their orbits. The number of such orbitual interferences or collisions - indicated, as the theory presumes, by luminous globes, shooting-stars, and aerolites - may startle some as an objection. But astronomy everywhere deals in numbers which surpass all common comprehension, yet are justified so frequently by certitude of proof, that we cannot refuse belief in others where the evidence is still incomplete. Arago, following one of Kepler's bold anticipations, has calculated that there may be eight millions of Comets having their revolution within the solar system. Meteorites, according to the present view, approach nearest to the character and condition of comets. The orbits of the matter thus revolving, (whether it be dense or infinitely attenuated) are probably not less excentric in outline, and have the same vast interplanetary spaces open to them. Numbers, then, need not perplex us here; and esppecially if admitting a view we shall notice hereafter, as to the seeming periodicity of the great showers of shooting-stars.

This cosmical theory of meteors in general has undoubtedly been gaining ground of late years, while other hypotheses have been stationary or retrograde. It has derived argument and illustration from the whole course of physical research during this period; with the effect of giving a new aspect to the phenomena, and associating them together as parts of a larger system and more general laws. We have placed a volume of the Cosmos of Humboldt among the works at the head of this article, because no philosopher has been 
more earnest in expounding and enforcing the opinion, that asteroids or aerolites are independent portions of matter in space; becoming luminous meteors or projectiles, when their orbits approach within certain distances of that of the earth. $\mathrm{He}$ avows, when leaving the subject, that he has lingered upon it with predilection (mit Vorliebe), and the whole course of his argument shows this to be so. Sir J. Herschel, an equal authority, expresses the same view as the only one which comprises, or adequately explains, all the phenomena; thus confirming the expression of Laplace (in his speech of December 1802) as to aerolites, that ' in all probability they come to us from the depths of the celestial space.'

There arise out of this theory various physical questions, some of which we cannot omit to notice. One of these respects the luminous and ignited condition of meteorites when approaching the earth. Though it seems certain that some alteration of state beyond mere change of direction is produced by this proximity; and though condensation of the air, from the extreme velocity of falling stones, might doubtless produce the heat, combustion, and explosion attending their fall; yet, from the elevation of many meteors, brilliant in light, above the recognised limits of the atmosphere, we are bound to suppose other causes also concerned. Modern science teaches us that ignition (viz. light and heat) occurs in various cases without the presence of air. In this case it may possibly be magnetical in kind; - a supposition authorised by the discoveries of the last few years, which make it probable that this great element is largely engaged even in the astronomical conditions of the universe. The paper recently published by Professor Faraday on the Physical lines of Magnetic Force, while marked by all the modesty of his genius, is profoundly suggestive of relations of this kind yet unexplored; and of forces pervading space in lines of action differing from any other of which we have 
yet cognizance. But we have no right to carry hypothesis farther, on a point to which even the ability of Poisson has been directed without any determinate conclusion.

Considering that all meteors involve the presence of matter in some form, and that aerolites show it by precipitation of solid masses on the earth, it is a question of interest what happens in the cases where we have not this direct result. The answer can hardly go beyond conjecture. Many meteors, even those containing solid matter, may be deflected in such degree towards the earth as to become luminous in a part of their course, yet still preserve their own independent orbits. Others, again, may undergo explosion or disruption during this contiguity, and throw down the same matters as those contained in meteoric stones, but under the form of powder or dust. Though this result is obviously more difficult of discovery, yet we have numerous proofs of the fact in the records of every age. Then, further, it is to be remembered how very small a proportion of the aerolites falling can come within human observation. The chances against any one stone being seen to fall on the earth are so numerous as to be hardly calculable, the mere interposition of night in our record of time entering largely into this question. The sight of such an event in fact is the exception, and not the rule. Weighing all this rightly, and taking into account also that the Ocean covers about threefourths of the globe, and that a large proportion of its landsurface is either unknown or uninhabited, we shall not be greatly surprised at the estimate of Schreibers that upwards of 700 meteoric stones, of various size, must fall annually upon the surface of our planet. It is only in the present state of science; when the most minute quantities are subjected to notice and calculation, that we could allude without ridicule to the fact of the increment thus made, and continually making, in the amount of solid matter of the globe. 
In theory this cannot happen without some certain amount of positive effect. In reality, we must consider the augmentation so small that it may be disregarded as a cause of any change in the motions or condition of our planet. M. Haidinger and Baron Reichenbach of Vienna have made some curious calculations on the subject; but they are wanting in those numerical facts which alone can give value to an estimate of this kind.*

We may notice here a curious remark of Olbers, that no meteoric stones have ever been found embedded in strata of the secondary or tertiary formations; and we have no direct proof, therefore, that any fell previously to the last great change of the earth's surface. This negative fact, however, cannot yet be admitted into argument. The careful examination of such rocks is still of recent date, and fossils of other kind have alone been sought for. Moreover, many meteoric stones are, so easily disintegrated, by the iron they contain passing into the state of hydrated oxide, that they may have become wholly incorporated with the earthy masses surrounding them. The chances, therefore, are exceedingly great against their detection in these rocks: but time may yet show, what must meanwhile be deemed most probable, that the phenomenon of their fall existed long before man had his place allotted him on this our globe.

We have in some part already adverted to the remarkable inferences and suggestions derived from the composition of meteoric stones. These bodies afford us glimpses into the history of matter foreign to the earth on which we ourselves live. They represent another domain of nature; yet connected with our own by the signal fact, also derived from them, that the matter is the same in kind as that which surrounds us here. Iron, as we have seen, largely predo-

* Mr. Haidinger calculates, on various grounds, that the weight of the aerolites which every year fall upon the earth may be about 450,000 lbs. 
minates over the other elementary substances entering into their composition; and this is associated occasionally with crystallised minerals resembling closely some of those found in our own rocks. While the materials, however, are thus alike, they differ much in their arrangement and proportions from any compound bodies hitherto known to us; and are of deep interest therefore as representing an aggregation, distinct from that of the earth, of the same elements existing beyond its sphere. Almost might we venture to call them specimens of planetary matter, since that which exists in the space intermediate between the earth and other planets may have the same relation to both. And if indulging in such speculation, we may go yet farther, and find argument in these facts for that great theory of modern astronomy, which regards all the planets as formed by the successive condensation of rings of nebulous matter concentric with the Sun;-the matter being the same but differently aggregated, from physical causes varying during the condensation of each planet.

Though no new element has yet been discovered in meteoric stones, we must not carry this negative beyond present proof. Analyses of other specimens may afford other results ; and we are not yet warranted in omitting any opportunity of further research. Besides the chance of new ingredients, such examination enables us to classify with more certainty these products of other regions of space, and thereby better to interpret the mystery of their origin and movements.

Another speculation still occurs in connection with aerolites. The researches of the last fifty years have disclosed to us some twenty new substances, hitherto undecomposed, and most of them metallic in kind. Certain of these substances exist only in single specimens ; - others are rare in occurrence and small in quantity. It has puzzled naturalists to conceive the purpose which matters thus scanty and insulated can fulfill 
in the economy of our globe. It is hardly probable, though possible, that these minute superficial specimens represent larger quantities in the interior of the earth. But is it not conceivable, looking to the composition of aerolites, that some of these elements, thus rare with us, may enter more abundantly into the composition of other planetary bodies? In the varying conditions of magnitude, figure, and specific gravity, as well as in the especial peculiarities of rings, belts, satellites, \&c., we have the certain proof of different modes of aggregation in each case. May we not reasonably suppose that this difference has extended to the kind and proportion of the elements, thus segregated and condensed from the vast material for which we vainly seek a befitting name? Speculations such as these do not fairly enter within the domain of science, but they border upon it; and now and then become the paths leading to new and unexpected truths. The objects of research are seemingly, indeed, too remote for access; but we have just seen how strangely some of them are brought within our reach. When a single small instrument, the polariscope, suffices to tell us the condition of light, whether issuing or reflected from a body a hundred million of miles distant in space, - or when the perturbations of certain known planets are made by the astronomer to indicate the place and motions of one yet wholly unknown, - it becomes difficult to despair of anything which time and genius may yet effect in the discovery of truth.

So far on the subject of Aerolites more especially; of which we have spoken thus fully, regarding this class of meteoric phenomena as best interpreting the others treated of in the works before us. It will have been seen already how closely all are allied, as well in various points of outward aspect, as in regard to the questions which concern their real nature and origin. One effect of this has been to render somewhat 
obscure to the untutored reader much of what even the ablest men have written on the subject. In the work of MM. Gravier and Saigey for instance, the history of Meteorites, though divided into periods, is perplexed by the continual passage from one class to another, and from observation to theory. We have ourselves endeavoured to avoid this perplexity, as far as it seemed to be possible under our actual ignorance of many of the relations of the phenomena. In proceeding now to speak of the meteoric globes or fireballs and the shooting-stars, we are following a provisional arrangement which may hereafter be cancelled; and are adopting names as we find them, since no better nomenclature has yet been brought to this part of science. The same thing has happened in other sciences; and such steps are natural in the history of all human progress.

The luminous globes are those in closest connection with the aerolites; inasmuch as we have various well-attested instances of stones - single or numerous - falling at the time of such appearances, and in sequel to explosions which would seem to rend asunder some larger volumes of matter. The following description of the ordinary character of the Bolide we take chiefly from our French authors, who correct some exaggerations of Chladni on this subject. They have claim to be considered an authority, since one of them, by incessant observation for several years, witnessed as many of these great meteors as the actual number noted during the same period by all other observers on the globe.

These meteors appear to move in the ares of great circles. They do not come equally from all points of the horizon, but affect certain principal directions. No movement of rotation is recognised in them. Their apparent disk is greatly enlarged by irradiation; and is occasionally seen to exceed the circumference of the full moon - which, at the distance of 110 miles, would give a diameter of about a mile. Their 
form is always circular. The amount of their illumination is much less than that of the moon. Their height is various, but often far beyond the limits of our atmosphere. They appear and disappear suddenly, without sensible change of diameter; sometimes bursting, but without noise; and often leaving a train of light behind. Their duration seldom exceeds a few seconds. Their velocity approximates to that of the earth, or other planets.

One curious fact relating to these meteors, and still more to shooting-stars, is, that they appear now and then to ascend; or to alternate in ascent or descent, as if new and opposite forces were suddenly brought into action. Chladni and others have sought explanation of this, either in resistance of the air compressed by rapid descent, or in the effects of explosion or ignition in the masses themselves. More recently, however, doubts have been suggested by Bessel - an authority entitled to much weight on this subject - as to the reality of these appearances. It is a point open to future observation and enquiry.

As is the case in every other part of science, the record of facts regarding these igneous meteors has become of late years infinitely more copious and exact. We have already noticed the extraordinary Chinese register, brought down from a very remote date. No other country, nor any age before the present, furnishes a like document. The first formal catalogue of remarkable meteors, of all classes, is that of a very eminent observer, M. Quetelet, published in 1837; and again with large additions in 1841. There soon followed the catalogue of Mr. Herrick in America, and that of M. Chasles, presented to the Académie des Sciences in 1841, - containing much curious retrospective information, and particularly as to the recorded falls of shooting-stars. The latest catalogue is that by Professor Baden Powell; - presented in series at the last five meetings of the British 
Association, and published in their Annual Reports.* Professing to be merely a continuation of Quetelet's Catalogue, and to form a nucleus for future collection, it is in itself a most copious and valuable register of these phenomena. We will not call it complete, because no record of these vagrant and fugitive appearances can be so. We do not, for instance, find noted in the Report for 1851 a very remarkable meteor, the appearance and disruption of which, on the 30th September, 1850, were witnessed at the Observatory at Cambridge, in Massachusets; and which has been fully described by Mr. Bond, the then distinguished astronomer of that university. $\dagger$ But many of these lacunce will be filled up; and meanwhile the catalogue is ample enough to furnish an admirable basis for future observation.

We have noted the frequent connection of these bright meteors with falling stones; and this is, in truth, the question of greatest interest regarding them. Are they really associated with some form of matter analogous to that of known aerolites, but which escapes detection, either by falling out of human sight, or by the passage forwards of the meteor in

* Since this article was published, a valuable addition to the history of Meteors and Aerolites has been made by a Report to the British Association for the Advancement of Science, in 1860; in which a Catalogue is given (carefully compiled, and largely illustrated by collateral facts and authorities) of all such appearances, from the commencement of the Christian era to our own time. Mr. R. P. Greg, the author of this valuable Catalogue, has printed it separately, as well as one or two other Memoirs on the same subject.

+ By a happy accident, I was present with Mr. Bond in the Harvard Observatory on the night, and at the moment, when this splendid meteor appeared and burst, followed by appearances not usually described. The most striking circumstances attending the phenomenon were, the long time (more than an hour) during which the nebulous light was visible after the explosion - the great distinctness of the nucleus, an elongated luminous space being projected, as it were, ahead of it - the perfectly cometary figure and aspect of the meteor a quarter of an hour after its first appearance, a fact strongly adverted to by $\mathrm{Mr}$. Bond - and the rotary motion of the luminous elongation amounting to nearly $90^{\circ}$ within twenty minutes, and producing a sort of whorl, resembling some of the nebulæ so beautifully depictured from Lord Rosse's late observations. 
its orbit without precipitation of its contents? Taking the question generally, we incline at once to answer in the affirmative. It must be admitted that stones have sometimes fallen from what seems to be a clear heaven; or with no other appearance than that of a small circular cloud suddenly forming in the sky. But these, as far as we know, are events of the daytime; and what is seen as a dark form under the light of the sun may appear a fiery globe in the darkness of night. If it be well proved in a few cases that these fire-balls exploding have thrown down stones upon the earth, the presumption becomes strong that analogous meteorical elements are present in all, whether precipitated or not. M. Saigey does not fully admit the relation of bolides and aerolites; but we believe the argument fairly to stand as we have stated it.

The subject of Shooting-Stars (étoiles filantes) separates itself somewhat farther from the phenomena already described, though still manifestly connected in various ways. The more important peculiarities here are the smaller size of these meteors; their infinitely greater frequency; the arcs they describe; their frequent occurrence in showers; and the observed periodicity in certain of these latter occurrences. The difference of magnitude is the least important of their characters; since we find every gradation of size, from the shooting scintilla of light to globes large as the moon. Those gradations, partially visible to any eye gazing into the depths of the sky on a clear night, are especially seen during the showers of stars just adverted to. The periodicity of some of these showers is the point of greatest interest in the enquiry; a research still very imperfect, but which time is certain to complete, and probably at no distant period.

The common aspect of shooting-stars needs no description. It was one of the earliest objects of science as directed to them, to determine their heights, duration, and velocity; and 
on these points we owe much to the persevering labours of Brandes and Benzenburg; an ample narrative of whose observations is given in the French work before us. Begun as early as 1798, they were continued at intervals of time and in different places, for a period of thirty-five years. Brandes died in 1834, just after he had received the account of that prodigious fall of shooting-stars in America on the 12 th and 13th November, which gave at once larger scope and better definition to all our views of these phenomena. To determine the points just mentioned, it was essential to have two observers at least, and a base of sufficient length for separate observation. Equally essential was it to assure the.identity of the objects seen; for which recourse was had to the exact time of appearance, as well as to the apparent brilliancy, swiftness, and length of train of each star observed. Observations strictly simultaneous were needful to success; and these could only be got by knowing the precise difference of longitude between the stations. The base first taken (two leagues in length) proved too short to furnish the parallax required. In 1801 the enquiry was resumed with the aid of two fresh observers; and four points were taken, the extremes of which, Hamburgh and Elberfeld, were about 200 miles distant. Here again it may be presumed that the separation was too great, since, out of a great number observed, only five shooting-stars could be actually identified. But this paucity of positive results is familiar to practical astronomy; and Benzenburg consoled himself in quoting the phrase of Lalande: 'Il n'y a que les astronomes qui sachent par combien d'observations manquées on en achète une seule qui réussit.'

During the remainder of the period we have named, similar observations were repeated by the same and many other observers in various parts of Germany; with different lengths of base, and aided by formulæ which Olbers and Erman had 
respectively suggested. Such, however, was the difficulty of establishing identity, that in 1823 (a year particularly devoted to this research) out of 1,712 shooting-stars actually observed, only thirty-seven could be conclusively regarded as the same seen at different stations. Nevertheless many valuable results were obtained, sufficient to indicate the general character of these meteors, and to associate them more closely with the fire-balls before described. Their height - varying, of course, in different shooting-stars, and at the moments of appearance and disappearance of each was found to range from 15 to 140 or 150 miles - (some statements much higher than these are made doubtful by the smallness of the parallax); their velocity to be that of planetary bodies, reaching frequently to thirty miles in the second. These conditions, together with the directions of the paths they describe in reference to the motion of the earth, suffice to assign their place in the planetary system, however small or attenuated the aggregations of matter thus presented to us.

A far more striking evidence, however, to this effect speedily followed, from the discovery of a periodical character in some of those showers of meteors, which at certain times startle the spectator by their number and brilliancy. The earliest suggestion of this arose from an extraordinary apparition of such meteors in New-England on the nights of the 12th and 13th of November, 1833; the description of which in much detail was given by Professor Olmsted of Newhaven and other observers. The asteroids composing this fiery shower graduated from the simple phosphorescent line of the shooting-star to luminous globes of the moon's diameter; - all of them conforming to one condition (the most important of the facts observed), that of issuing from the same point in the constellation Leo; and continuing to proceed from this point, though the rotation of the earth during the 
progress of the phenomena had greatly changed its apparent place in the heavens. The value of this observation was at once recognised. Sporadic shooting-stars are observed to traverse the sky in all directions. But these multitudinous meteors of a night, in their radiation from one point, showed a common origin and the approach of the earth in its orbit to some other revolving volume of matter, visible only through the changes made by such approximation.

Intelligence of this event, confirmed by other observers in different localities, awakened a new and keener interest in the subject. Reference was made to the same date in antecedent years, and several instances discovered in which about the 12th of November extraordinary falls of shooting-stars had occurred; - the most remarkable that described by Humboldt and Bompland in 1799, which occurred to their observation at Cumana, and was seen very extensively over the earth. Earnest expectation also was directed towards the future. On the night of the 12 th November, 1834, shootingstars were very numerously seen by the same American observers, and proceeding from the same point in the heavens; but the light of the moon rendered the results partial and uncertain. In succeeding years the phenomena were more vaguely seen or altogether absent; except in 1837 and 1838, when they recurred, but more partially as to localities. In the former year, for instance, they formed a striking spectacle in some parts of England while scarcely visible in Germany. Though M. Saigey imputes much exaggeration in numbers to the Transatlantic reports, they have been admitted by the most eminent men of science-Arago, Biot, Herschel, Humboldt, Encke, \&c., — as fully proving the periodical return of certain groups of asteroids, or of the matter generating them. To Encke we owe the calculation that the point in Leo, from which these November meteorites issued, is precisely the direction in which the earth was moving in its 
orbit at this particular time - a fact, the value of which in relation to their theory will readily be understood.

But the eager attention now given to the subject speedily evoked other results. It was found, as well from prior record as from present observation, that November was not the sole period of recurrence of such phenomena. Tradition, both in England and elsewhere, pointed out the 10th of August, St. Lawrence's day, as frequently marked by these fiery showers. In some parts of Germany the belief ran that St. Lawrence wept tears of fire on the night of his fête. An old monkish calendar, found at Cambridge, reciting the natural events which belong to different days of the year, designates this day as one of meteors (meteorodes). We find a curious notice by Sir W. Hamilton of such a shower, as he witnessed it at Naples on August 10, 1799. In 1839 these August asteroids were very remarkable; and it has been distinctly ascertained that they proceeded from a point in the heavens between Perseus and Taurus, in direction towards which point the earth traverses a tangent to her orbit at the time - a very. striking concurrence with the facts just stated respecting the November phenomenon. Further research has indicated other times of the year-April, July, and December - as marked by like periodical appearances; but the evidence is less distinct, and does not go further than to justify the demand for future and multiplied observations.

The admission of these wonderful facts created instant enquiry into their cause. No theory was seemingly tenable which did not recognise in some form a revolution round the Sun of the matter composing or evolving these asteroids. Professor Olmsted and other American naturalists, fresh from the spectacle that had been before their eyes, first took up the question. The observers, collecting all the facts, deduced from them the existence of a nebulous cloud or mass of meteoric stars, approaching the earth at particular periods of 
its revolution, under conditions as to time, direction, and physical changes from proximity, which we have not space to detail. The speculation that this meteoric cloud might be part of the solar nebula known under the name of the Zodiacal Light, was adopted and enlarged upon by Biot, in a memoir read before the Académie des Sciences in 1836 . The first exact observer of the zodiacal light, Cassini, had long before inferred that it consists of diffused planetary matter. It is shown by Biot that on the 13th of November the earth is in such relative position that it must necessarily act by attraction or contact upon the material particles of which this nebula is composed, producing phenomena which we may consider to be represented by these meteoric showers. He carries the same theory to the explanation of the sporadic shooting-stars of ordinary nights, by supposing that the constant passage of Mercury and Venus across the more central regions of this nebula may have dispersed innumerable particles in orbits very little inclined to the ecliptic, and so variously directed that the earth encounters, attracts, and renders them luminous in every part of its revolution.

Objections have been raised to this theory, and it remains without any fresh confirmation. But under any form that can be given to the question before us, it seems needful to assume for its solution the existence of matter (revolving either in zones or in separate masses and groups) constituting the material of these asteroids. The hypothesis of matter (cosmical matter it has been called) - thus arranged; having periods of revolution more or less regular, and intersecting. the orbit of the earth in certain points at certain times, has been adopted by Arago, Herschel, and other eminent astronomers; and the conception of a zone or zones of such matter is admitted as best fulfilling on the whole the conditions of the problem. Under this view of revolution, already expounded in a more general way as applied to 
meteors of every class, we obtain the only clear notion of a cause of periodicity; - the law being the same which governs the planetary system at large, and even the most excentric motions depending on the great principle which maintains general order throughout the universe.

It must be admitted that this theory materially changes our manner of viewing the interplanetary spaces around us. No longer regarded as a void - or filled solely by a subtle ether, imponderable and unseen - these spaces now present themselves as occupied in various parts by matter apparently of the same nature as those of which our globe is composed; - but either not yet aggregated into planetary forms, or detached from planetary bodies previously existing. If adopting this idea of meteoric zones or rings, we must necessarily admit at least two such in explanation of the separate, but similar, phenomena of the August and November showers. Many questions, however, still remain open; such as, whether these zones are of uniform composition and arrangement? - whether there is any proof of a progression in the line of nodes, or of oscillation from perturbations?whether we may attribute to them the occasional obscuration of the Sun for short periods, which we find on frequent record? - and on what physical causes depend the luminous globes and shooting-stars which emanate from them on approaching the earth ?*

Other questions there are, awaiting the possible solution of the future, some of which our readers will already infer. To explain the appearance of single meteors, always so sudden, often so brilliant - as well as the more substantial phenomenon of falling stones - must we not suppose detached portions of matter, equally revolving as the zones which

* The most authentic statement as to the August zone is that of Professor Twining, founded on his own diligent observation of the phenomena occurring in this month. 
pour forth periodical showers, but each with an independent orbit of its own? What physical causes can have produced such separate accumulation or consolidation of these portions of matter? Both analogy and the known laws of the mechanism of the heavens, furnish a certain explanation of zones or rings, but we have no similar aid to our understanding of these insulated masses moving in space. Are they residual merely upon the consolidation of larger bodies? or must we regard them as detached by some unknown force from bodies already consolidated? The fragmentary character of aerolites as well as the materials composing them, might suggest the latter idea, and the numerous group of excentric planetoids between Mars and Jupiter give sanction to it; but we have already followed out the argument derived from these sources, and seen how much is wanting to its certainty and completion,

Before closing this article we must make more particular mention of the valuable work of M. Saigey, recording, in sequel to an Historical Introduction, those long series of observations by M. Coulvier-Gravier, in which latterly the writer himself took an important share. We prefer such separate notice, both because these researches are little known in this country, and because their purport will be better understood from the relation already given of the previous state of knowledge and opinion on the subject. We must begin by stating that M. Saigey acquiesces only very partially in the conclusions we have described as adopted by the most eminent scientific men of the age. He contends that these conclusions are premature; based in many points on doubtful or insufficient observations; and pressed forward by astronomers relying too much on analogies drawn from their own more certain science. $\mathrm{He}$ asserts that longer research into facts is needful to any theory on the subject; 
and justifies this by the record of results which show that other and new conditions must be added to the theories of meteoric phenomena now received. Of the more remarkable of these results we shall give a short summary; such as may enable our readers to judge of their nature and bearing on the argument.

Observations on shooting-stars and other meteors were begun by M. Coulvier-Gravier at Rheims as early as 1811, under electrical and other theories of their origin, which he afterwards abandoned. It was not, however, until 1841 that, at the suggestion of Arago, he began carefully to register their number, times of appearance, and direction in the heavens. In 1845 M. Saigey associated himself to his labours, and aided greatly in generalising and giving method to the results. In a period of 42 months, between 1841 and 1845, there were 5,302 shooting-stars recorded; - seen during 1,054 hours of observation. The number would doubtless have been much greater but for the interference of the moon, which, when full, effaces nearly three-fifths of the stars otherwise visible. An estimate made, with allowance for this cause, brings out the mean horary number of 6 ; the actual mean number seen per hour being $5 \cdot 6$. The passing obscuration by clouds makes another void in the calculation, the amount of which it is difficult to estimate.

But this general horary mean loses its interest in another more curious and unlooked-for result of these observations, viz. the variations found to exist at different hours. With rare exceptions, the number of visible meteors increases as the night advances; and this at all times of the year, and with regularity enough to furnish the basis of tables for each successive hour of the night. A few instances we give from different hours between evening and morning. In the evening from 6 to 7 o'clock the mean number of stars falling is $3 \cdot 3$ - from 9 to 10 o'clock 4 - from 11 to 12 o'clock 5 - 
from 2 to 3 o'clock in the morning $7 \cdot 1$-from 5 to 6 o'clock 8.2. And this gradation is maintained also in the case of the meteors of periodical recurrence.

Equally remarkable is the result as to the monthly or annual variations of these phenomena. A laborious reduction of observations has furnished a table expressing the monthly mean of the horary number at midnight. This table shows a singular disparity between the first six months of the year and the last; the mean number of shooting-stars in the former being only 3.4 in the hour, in the latter rising as high as 8 ; - that is, a smaller number when the earth is moving from perihelion to aphelion, or receding from the sun - a much greater number in the other six months, when it is advancing towards its peribelion. The transition is rapid from one of these conditions to the other. In December the mean number in the hour is $7 \cdot 2$ - in January only $3 \cdot 6$. In June $3 \cdot 2$ - in July $7 \cdot 0$. It is well worthy of note that the two maxima in the table occur in Auguist and November; thus according exactly in date with the periodical showers we have described; and with the further concurrence of fact that these maxima are not present every year. In 1842 the mean for August was $11 \cdot 9$ - in 1844 only 5.4. In 1842 the mean for November was $11 \cdot 3$ - in 1843 it was $5 \cdot 4$.

Another section of the enquiry before us regards the direction of these shooting-stars. Without entering into the details, we may remark the general conclusion that almost exactly the same number come from the north and south conjointly, as from the east and west; but with this diversity, that, while the number is nearly the same from north and south, the number coming from the east much more than doubles that from the west. The amount of the diversity, however, differs in different years. This copious accumulation of facts afforded other curious results as to the length of the visible trajectories, the position of the centre of the 
meteors, \&c. The shooting-stars comprised between NNE. and NE. have the longest visible course, the mean being upwards of 15 degrees; - those between WSW. and SW. are only seen through about 11 degrees. Whatever the time of year or hour of night the line is one of descent towards the horizon. Out of 5,302 fifteen only were seen to describe curves.

The estimate of our authors as to the height of shootingstars places their point of appearance at from 20 to 50 or 60 miles above the earth. Their relative size, colour, and manner of apparition were carefully observed. Of Bolicles (luminous globes) eight were noted during 42 months, three only of which burst, and these without any noise of explosion. Of the proper shooting-stars 80 were registered as of the first magnitude, having the apparent size and lustre of Venus or Jupiter. The others were classed down to the 6 th magnitude, corresponding to-the 5 th of the fixed stars. The colour, especially of the largest, is generally a pure white. Those of red colour are rarer; but remarkable as seeming to be slower in movement, and not leaving trains of light behind. Some occur of bluish colour, but still more rarely.

We find it necessary to abstain from further details, but we believe we have said enough to show the value of these researches. They clearly suggest many important considerations hitherto little regarded; and some of these, as already remarked, at variance with the conclusions now generally adopted. We must admit that a revision of these conclusions is required; and their adaptation, if such be possible, to the new facts brought before us. Allowing the authenticity of the latter, we are bound to say that no theory of meteoric phenomena can be complete which does not include and explain the horary and annual variations just described. They are problems of high interest, but of very difficult comprehension. And while thus recording the most recent researches in this 
part of science, we must repeat our opinion, that a much larger basis of observation is required to raise the phenomena to the class of astronomical facts. Time alone is capable of affording this. We cannot follow the fleeting meteor as we do the planet, or even the more excentric comet, night after night on their paths. But modern science has taught us to derive certainty from averages as well as from more direct observation; and the multiplication of insulated facts, if exact and authentic, is sure in the end to conduct us to the truth desired, or as near to it as human powers are permitted to approach. Happy those who can detach themselves at times from the turmoil of the busy world we inhabit, and find repose among the more silent wonders of the universe without! - a contemplation scarcely disturbed even by these flaming ministers of the sky, which now no longer come to affright mankind, but to enlighten and enlarge our intelligence and power.

\section{POSTSCRIPT.}

It may be well to add to the foregoing essay a slight notice of what has farther been done to augment our knowledge on this curious subject. The first fact is, the great increase in the number of aerolites, as now collected and authenticated in the different Museums of Europe. In the British Museum alone, the number has been at least doubled within the last four years by the zeal and intelligence of Mr. Maskelyne, who has sought and obtained them from every part of the world. On the shelves of this great establishment - rich to overflowing in its every department - there are at this time (1862) 83 specimens of aerolites of various size and weight, and 62 specimens of meteoric iron. One cabinet alone contains a group of six splendid specimens, of known origin as aerolites. The largest of these, which fell in 
1857 at Madura in the Madras Presidency, weighs 138 lbs. Another is the famous Yorkshire stone, the fall of which first fully awakened attention to this subject. The collection at Vienna is somewhat more numerous than that of our Museum; but the specimens are inferior in average size.

No new element, as far as we know, has been recently discovered in these bodies. But considerable interest has lately been excited by the observation, in three or four of them, of a bituminous matter, or hydro-carbon, forming a part of the substance of the aerolite. Berzelius first gave some intimation of this fact in the case of a stone which fell near Alais in France. But Wöhler has more recently detected the same material in a large aerolite which was seen to fall at Bokkevelde in the Cape Colony; and similar observations have reached us from other quarters. The interest attached to this fact depends on the assumption that such hydro-carbonous matters are of vegetable origin; and that vegetable life must therefore have existed wherever aerolites containing them are formed; - a very striking conclusion, if authenticated to our belief. Doubts, however, have arisen whether these ingredients may not be the product of vegetable matter on the place of fall, thus changed by the heat of the meteoric stone, and becoming embodied in its crust. This question, like so many others, must be submitted to future and exact observation of all the facts concerned.

Allusion has been made (page 289) to that curious enquiry - so characteristic of the aims and objects of modern science - whether the conditions of the earth, as a planetary body, may not be changed in time by the continuous fall of aerolites upon it from age to age? The same bold speculation has been extended even to the Sun, the enormous mass of which must powerfully draw to itself the various loose forms of matter in inter-planetary space, or even perhaps in the sidereal space, through which it is moving. Attempt 
has been made (and this by more than one philosopher of the day) to reach another inference, founded on the laws which govern matter and forces on our own globe. The Sun is ever radiating into space, from each point in its vast circumference, an amount of heat hardly to be estimated by numbers or thought. How is this loss (for so upon every theory of Heat we must call it) to be repaired? It is difficult to conceive any other source of supply than from this very surrounding space, which contains, as we have seen, matter of different kinds, capable of being attracted to the planetary bodies of our system. Every sudden impact of matter upon matter evolves heat, and this in proportion to the violence of the impulse. The hypothesis in question, adopting the estimate of 300 miles a second as the final velocity of a body falling on the Sun, supposes that an amount of Heat may be thus generated, sufficient to repair the waste ever going on. It is a theory, we are bound to say, sanctioned rather by the want of any other plausible explanation than by its own intrinsic evidence. But it may fitly be mentioned in connection with the subject of Meteorites, and as illustrating the arduous speculations of the philosophers of our day. 


\section{HUMBOLDT'S COSMOS - SIDEREAL ASTRONOMY.**}

[QUARTERLY REVIEW, No. 87.]

SINCE we reviewed the first volume of this work in 1846 , Baron Von Humboldt, laden with years and well-earned honours, has published two additional volumes. We feel it incumbent upon us to bring the work, thus enlarged though still incomplete, again before the notice of our readers. This we do, as well from a regard to the high eminence of its author, as because it forms an exposition of the general state of physical science, brought yet nearer to our own day by a philosopher of large views and knowledge matured by a long life of active observation;-one equally capable of generalising what has been already done, and of casting a philosophic eye upon that 'ocean of undiscovered truth,' which still lies open before us.

What we consider peculiar to Humboldt is the singular extent and diversity of knowledge which he brings to every subject of enquiry. We cannot name any traveller equally gifted with this large comprehension, possessed and brought into active exercise at the very outset of life. A striking example of his copiousness of research occurs in the earliest part of the personal narrative of his travels. Approaching

* Cosmos. Sketch of a Physical Description of the Universe. By Alexander Von Humboldt. Vols. ii. and iii. Translated under the superintendence of Lieut.-Colonel Edward Sabine, R.A., V.P. and Treas. R.S. London, 1850, $1851,1852$. 
the Canary Isles (the first point at which he touches), he enters into a long discussion on the currents and winds of the Atlantic, that great valley of waters dividing the Old from the New World. The sight of the stupendous Peak of Teneriffe leads him to a dissertation on those various conditions of figure of the earth, figure of the object, refraction, \&c., which determine the visibility of objects at different distances. Six days at Teneriffe, including an ascent of the Peak, furnish materials for half a volume; in which are blended geology, botany, and zoology - vivid descriptions of the island scenery - theories of volcanic phenomena - questions as to the temperature and chemical composition of the air at different heights - the history of the Canaries - disquisitions on their discovery by the ancients, and on the origin and primitive language of the Guanches, their earliest known population. Many of these topics have been enlarged or corrected by later research; but, as handled by Humboldt at this period, they well mark his early vigour and aptitude for such enquiries.*

Regarding him as a writer merely, this exuberance of knowledge, and his nimia diligentia of illustration, almost pass into a fault, if we might apply the term to qualities thus valuable and rare. Digressions may readily be excused where they bring fresh life and vigour to the subject, and suggest new relations to the mind. But, even under this view, we must consider the tendency in Humboldt's case to be one of excess; and we notice it the rather from finding proofs of the same discursive method in the work before us; in which the topics, from their vastness and variety, require constant compression, and an adherence to that proportion of parts which is essential to the unity of the whole. Where the

* This article having been in great part written during a visit I made to the Canary Isles in 1853, with the rolumes of Humboldt in my hands, I was naturally led to the example given above. 
Universe, which we must receive as the proper rendering of Cosmos, is the object placed before us, we have some right to expect that the grandeur of the design should be sustained in the execution.

In our former article we made some remarks on this subject; commenting upon a certain vagueness which pervades the whole conception of the work; and a tendency to repetition and digression, injurious more or less to the harmony of the scheme. These comments are confirmed and justified by the volumes now before us. In adopting the title of Cosmos, M. Humboldt has exposed himself to perplexities which pursue him through every part of his work. $\mathrm{He}$ is haunted, one may fairly say, by a spectre of his own creation. He has invoked a vast and vague name, which sometimes he seeks to curb and limit by definitions, at other times to enlarge and exalt. At the risk of appearing presumptuous we must express our doubt whether he ever fully defined the term of Cosmos to his own mind. A grand and spacious idea had been long before him (in unbestimmten Umrissen, as he himself expresses it), congenial to the temperament of German thought, and according well with his own large knowledge, and his desire to concentrate the labours of a life in one great closing work. He sought to mark by the name the magnitude of the conception.* But the conception itself is beyond the power of adequate fulfilment, even by one possessing the resources of our author. The Universe, as expressing all the material phenomena of nature (and Humboldt has superadded other topics having relation to the human faculties and progress), is too vast a theme for a single man or a single work. Treated upon one plan, it

* Perhaps his tersest definition of the Cosmos is the phrase in the Preface, in which he describes Nature as ' a whole, moved and vivified through inward powers' (ein durch innere Kräfte bewegtes und belebtes Ganze). And yet it will be felt how far this, even as an outline, falls short of conveying any clear conception to the mind. 
becomes a loose and almost metaphysical abstraction, upon the opposite plan, an embodiment of facts and details so various and endless as utterly to set at nought all power of compression or scientific arrangement. The expression of Seneca, designating his idea of the Divinity of the Universe, ' quod vides totum et quod non vides totum,' has, in some points, close relation to our author's conception of the Cosmos; which is here and there denoted in terms savouring more of the school of Fichte and Schelling than of the sober severity of modern science. We presume it likely that Humboldt had before him the idea, if not the words, of D'Alembert, — ' L'Univers, pour qui saurait l'embrasser d'un seul point de vue, ne serait qu'un fait unique, et une grande vérité ' - a phrase intelligible in no other sense than as it points to that unity of creation and of the Divine power, which, while establishing through the elementary forces mutual relations among the most remote bodies of the universe, has equally designed the most subtle atomical relations of matter, and those exquisite organic textures which minister to the functions of life in its numberless forms on our own globe.*

The difficulties and incongruities resulting from this struggle between the abstractions of a name, and the real genius and acquirements of the author, are apparent in the methods and construction of the whole work. We see them in the frequent

* The conceptions of Goethe, as embodied in his strenuous verse, were doubtless also present to our author's mind in forming the scheme of the Cosmos:-

- Und hier schliesst die Natur den Ring der ewigen Kräfte,

Doch ein neuer sogleich fasset den Vorigen an;

Dass die Kette sich fort durch alle Zeiten vorlänge,

Und das Ganze belebt, so wie das Einzelne sey.'

The 'Traité du Monde' of Descartes, and the 'Cosmotheoros' of Huyghens, may occur here to some of our readers. But the first work was never published entire; the second was little worthy of the name of Huyghens; and neither of them could suggest anything to the mind of Humboldt, so well exercised in the sounder science of the present day. 
recurrence of M. Humboldt to definitions of his plan and explanations of the idea of the Cosmos; seemingly as much to satisfy and guide his own mind, as to direct the intelligence of his readers. Largely though this matter is treated in the Introduction to the first volume, we find a recurrence to it preceding the chapters entitled 'Epochs in the History of the Physical Contemplation of the Universe.' Even to the third volume there is prefixed a new Introduction; in which, while reciting the purport of the former volumes, and the object still before him, he makes a distinct admission that the scheme is too large for a single hand; and anticipates, rather by apology than vindication, some of the objections we have ourselves urged to the conception of the work.

We find further evidence of this shadowy and undefined conception of the Cosmos in the peculiar phraseology pervading the whole work; - less at variance, indeed, with German than with English habitudes of thought and language; but, under any view of it, much more vague and mystical than befits a scientific treatise of our day. We might quote in illustration such expressions as 'domain of the Cosmos,' 'science of the Cosmos,' 'recognition of the Cosmos,' ' history of Cosmical contemplation,' 'Cosmical space,' 'Cosmical life,' and many others of like kind occurring in these volumes. The translator rightly renders them to us as he found them; but they might be well exchanged for terms of more common and intelligible use.*

The injurious effect of the title and scheme of the Cosmos is strikingly felt in the distribution of the subjects of these

* With the exception of what relates to the imperfection of knowledge and personal observation, we might almost be led to apply to the Cosmos the commentary its author makes on the Natural History of Pliny:-

- There floated upon the mind of Pliny a grand and single image; but, diverted from his purpose by specialties, and wanting the living personal contemplation of nature, he was unable to hold fast the image. The execution remained imperfect, not merely from haste and frequent want of knowledge of the objects to be treated, but also from defective arrangement.' 
two volumes. After an impartial perusal of the explanation offered in the Introduction just commented upon, we are still compelled to state that the arrangement adopted involves both incongruities and repetitions. We doubt not that some of these difficulties have arisen to Baron Humboldt from the manner of his publication. Physical science in all its branches has been advancing with gigantic steps since the first part of his work was given to the world. Much has been discovered that is new, both in facts and in the laws governing them; - numerous errors have been corrected; the methods and instruments of enquiry have been unceasingly improved; and science is made to yield practical results to the uses of man much more largely than ever heretofore. A year now is equivalent to ten years at any former time of its history. Our author is far too zealous an observer of this progress, and too acute in his appreciation of it, to allow these things to pass without record. Neither age, nor courtly favours, have rendered him indifferent to what is going on in the world of science around him. He lives in the atmosphere of Berlin, teeming with active experimental researches, and bold speculation founded upon them. $\mathbf{\Lambda}$ natural desire for the completeness of his undertaking is further fostered by an intellectual temperament prone to the collection of facts, and to the establishment of those great relations which give them their value and efficiency. Later volumes, coming out after the lapse of years, are thus made to supply the deficiencies of those which have gone before. We do not wish to speak reproachfully of that which must be considered inevitable, if not indeed laudable, in the conduct of the work; but that it is an imperfection in the scheme, and destructive of its unity and fitness of proportion, can hardly be denied.

It is with regret that we have found ourselves obliged to make these preliminary remarks. But, dealing conscien- 
tiously with a work which comes out under the auspices of a great name, we cannot refrain from repeating our conviction that it is embarrassed by a title of needless abstraction - that the principle and plan of execution have never been clearly defined - and that the publication by instalments has led to a repetition and disproportion of parts, in what professes to be a simple and connected whole.* The attempt, in fact, is one above the power of present fulfilment; and peculiarly inappropriate at a time when physical science is every year changing its aspects and enlarging its dominion. There is value, indeed, in every work which clearly expounds the stages of this progress, or so associates them as to suggest new objects of enquiry. But, out of the domain of mathematical methods, nothing must yet be regarded as certain or complete; and the Cosmos of Humboldt, in assuming a character which even he fails to realise, involves both omissions and redundancies, which in the fairest spirit of criticism it is impossible not to recognise and regret.

:- Proceeding now to analyse these two volumes in detail, we find the first part directed to the consideration of the 'Incitements to the Study of Nature;' and distributed under the three heads of 'Poetic Descriptions of Nature,' 'Landscape Painting,' and 'Culture of Characteristic Exotic Plants.' This disquisition-if indeed admissible at all into a physical description of the Universe - is strangely placed between

* Without incurring any charge of national partiality, we may be allowed to refer here to the volume of our distinguished countrywoman, Mrs. Somerville, ' On the Connection of the Physical Sciences,' as including, under a lucid arrangement, all the essential parts of the Cosmos. Her title is less assuming; but it embodies a much clearer and more important conception;-that. mutual relation and inter-dependence of the sciences, the knowledge of which is the highest end and aim of physical philosophy. The comparison of this work with that of M. Humboldt offers certain contrasts, curiously expressing the intellectual differences which produce them ; - the more curiously, because these differences are not such as might be looked for from the respective sex of the writers. 
two separate treatises, descriptive in different degrees of detail, of the physical phenomena of the Heavens and the Earth. To us, we confess, this part of the second volume has the air of a separate dissertation, alien in date and substance from the materials with which it is now incorporated. Unless the term Cosmos were interpreted as including the history of man in his whole moral and intellectual being (in which case this part of the work would be very inadequately fulfilled), we cannot see the fitness of this treatise on poetic descriptions of nature, on landscape-painting, and on the culture of exotic plants. It is the most reasonable belief that these chapters are an excrescence which has grown upon the original plan of the author.

Looking to the Chapters themselves, apart from such considerations, we find in them a very agreeable collection of passages, illustrating the genius and habits of different races and communities of men in relation to the world of nature. We are very ready to acknowledge the pleasure afforded us by the examples so selected; but our author appears to assign to them a higher value and interest than we believe them actually to possess. Even admitting, what can hardly be conceded, that we may take the natural taste or genius of two or three writers as a criterion of the like qualities in a nation or great community, it may fairly be questioned whether there is any novelty in the inferences sought for; viz., that even in the earliest ages, and among every people of mankind, there has been a perception of natural beauty and sublimity - a desire to express such feelings in language or other form of representation - and a great extension and refinement of them by the culture of modern times and civilised life. The fact is one so generally recognised, that examples were scarcely needed to justify or enforce it. Without embarrassing ourselves by definitions or theories of the Sublime and Beautiful in Nature, we feel it to be certain that 
the Creator has gifted Man with a capacity for the pleasurable perception of these qualities in things created. The existence of an innate moral sense has been the subject of various doubt and controversy. But none can occur as to the faculty of which we now speak. It forms part of the physiology of the external senses. There is equal proof of its existence as of that of the intellectual faculties; and the only thing which can create doubt or difficulty is the disparity in the degree of this endowment in different individuals and communities, and the vast improvement of which it is proved to be susceptible from artificial cultivation. But the difficulty is precisely the same as to the intellectual part of our nature. For without being disciples of the German phrenology, we cannot but recognise in common with all the world, those original diversities of mind, those peculiar endowments and propensities, which determine the character and genius of the individual; and which, under certain conditions, and under laws governing the propagation of the human species, become the germs of national character, and develope in classes and communities of mankind qualities which were peculiar to individuals in their origin and earlier progress.

Much might be written, and much speculated, on this latter topic; but we allude to it only in connection with the part of the Cosmos now before us. We think that our author has in reality narrowed his views on the subject by this large collection of particular descriptive passages, from different languages and successive ages. The chief value of such a collection must be the discrimination, as far as possible, of the peculiarities of each age, race, or community; and of the causes whence these originate. To a certain extent this object is fulfilled, but we cannot say very satisfactorily. A few translations, derived from Persian, Hindoo, or Chinese poetry, serve scantily to illustrate the peculiar temperament of these vast races in relation to the beauty and wonders of 
the world of nature. Baron Humboldt, however, is ample, just, and eloquent in his appreciation of the sacred poetry of the Hebrews. He speaks, and with much truth, of the 104th Psalm as a 'picture of the entire Cosmos.' The most casual and careless reader of the passages quoted from it will indeed see how marvellously they outshine the minora sidera of the other examples; not less in the grandeur of the objects individually presented, than in the comprehensiveness of this great picture of nature in its relation to the Creator of the whole. Our author alludes in terms of like admiration to other portions of the Psalms and Book of Job; and quotes with full assent what is said by Goethe of the Book of Ruth, that 'we have nothing so lovely in the whole range of epic and idyllic poetry.'

In treating of the temperament and culture of the Greeks and Romans as regards the perception of natural beauty, he cites various passages more or less familiar to the classical reader. Numerous others might of course be added from this rich storehouse of poetic conceptions and imagery. We confess, however, we think our author too generous in his estimate of Cicero's love of nature and rural retirement. Particular portions of his epistles and philosophic.works may seem to justify this; but in some of these we are compelled to recognise political discontent; in others, the love of his own eloquent and beautiful descriptions. The affections of Cicero were really in Rome, even amidst the turbulence of those distracted days of an expiring Republic. 'Urbem, urbem, mi Rufe, cole, et in istâ luce vive,' is his earnest exclamation to a friend; and one more genuine, we fear, than any eulogium on his Tusculan or other villas.

But amidst this exuberance of poetic passages, we yet have before us the extraordinary fact that neither Greeks nor Romans ever reached the just perception of what we term landscape; - that grouping of objects by form, colouring, 
and other more refined associations, which has now become a source of such various delight, both in the direct contemplation of nature and through the medium of works of representative art. The Greek poet, for the most part, takes his objects from nature singly, or under some very simple combination; and generally for purposes closely connected with human feeling or action. They are not brought forward, as in the passages of modern descriptive poetry or prose, explicitly to place a landscape or group of natural objects before the eye of the imagination, but to illustrate or invigorate those narratives, of which man is the chief object and centre.*

Our author has alluded to this fact in his chapter on Landscape Painting, but more cursorily than we should desire. We consider it (especially as regards the Greeks, to whom Rome was the debtor in art though an illustrious one) as one of those singular anomalies which perplex all common calculations of probability. It is easy to state that in classical antiquity the taste and feelings were mainly directed to representations of the human form, or to the perfection of architecture in its various styles. This is doubtless true; but it yet leaves open the question, why this exclusiveness existed? -how a people like the Greeks, keen in their perceptions of grandeur and beauty, animated and vigorous in the exercise of all their faculties, and capable of works so exquisite in poetry, sculpture, and architecture, should have failed in

* M. Humboldt notices, with proper commendation, Ællian's description of Tempe, as the most detailed description of natural scenery by a Greek prosewriter which we possess. Livy's description of the same celebrated valley merits similar commendation; as well as his picture of the great plain of Thessaly suddenly bursting into view from the pass over Mount Othrys. The accuracy of both these descriptions we can ourselves attest from personal observation; and the proof they afford, in common with many other passages, of Livy's strong perception of the objects of landscape. But the general fact, as to the deficiency of this perception among the ancients, we believe to be as stated above. 
reaching that art of landscape delineation, which has attained such variety of excellence in modern times. Painters they had; and the great names of Zeuxis, Apelles, Parrhasius, Polygnotus, \&c., have descended to us ; their powers attested by the prices of their works, and the universal admiration they obtained. But the subjects chosen for painting seem, with little exception, to have been the same as those of Grecian sculpture, viz. the delineation and colouring of the human figure and features, either singly or grouped in action. All other objects were regarded as subordinate to these, and we are obliged to believe that they scarcely went beyond the elements of perspective in their paintings. A long series of ages, reaching to the confines of our own time, was required to attain that excellence of a new art, which often inverted the style and feeling of the ancients, by making man and his works mere accessories to the delineation of nature.

Returning from what might seem a digression but for the sanction of our author, we may advert briefly to the remainder of his second volume, which, under the singular title of 'Epochs in the History of the Contemplation of the Universe,' includes a history of the progress of discovery from the earliest times; as well on the land and oceans of our own globe, as in the celestial spaces so marvellously explored by the labour and genius of man. He arranges the whole under seven epochs; - a classification somewhat arbitrary in its character, and liable to various objections in its details. But we admit the need of some arrangement, and we know not that any better could have been proposed. We may strongly recommend these chapters to all who love to follow the line of human advancement, from the feeble and uncertain steps of its infant state to the gigantic march of our own time. Baron Humboldt's narrative is vigorous, impartial, and complete within the limits he has assigned to it. The most accomplished traveller himself of modern times, 
he is entitled and best able to record that progress of human discovery which, from the maria clausa of ancient history, has carried men forwards over the oceans and continents of the total globe; has given to the then remote and barbarous Britain the present power of sending forth hundreds of ships annually to the gold-bearing lands which form our antipodes; and has created, by aid of the great natural agents around us, new faculties of motion and speed, transcending the most romantic fairy-tale of former generations.

In the early and doubtful part of this narrative we find some conclusions stated with greater assurance than seems justified by the evidence we possess. We might adduce as a single case the interpretation given to the expedition of the Argonauts;' the basis of which, apart from its mythical garb, is considered to be 'the fulfilment of a national desire to open the inhospitable Euxine.' Frequently, too, we are struck with the affectation of using obscure terms when more simple ones are at hand; and a fondness for new forms of phraseology without any obvious requirement. But we are bound to accept the individuality of Humboldt's style as it occurs throughout all his writings; - one more picturesque and imaginative than is common in works of science, and abounding in original phrases fitted to express new collocations of facts or ideas. He has enough of the German temperament to take delight in these innovations, to which he is in truth fairly entitled by the many new relations he has himself pointed out in every part of nature. And we would repeat again that there is very high merit in this part of the work; which marks out, more clearly and compressedly than had been done before, the ages and races chiefly concerned in the progress of discovery, the events which have especially contributed to it, and the individuals whose ardour and intelligence have made them foremost in this great career. It is fit that these things should be historically recorded. 
As might be expected, from its forming the scene of his own earlier labours, the discovery of America has a strong hold on Humboldt's mind and imagination. He contemplates it as a natural result of the growing philosophy of the age. In a disquisition of some length on the era of Columbus, we are amused by the scholastic turn given to the subject, and by finding not merely the names of the eminent persons preceding or belonging to this age (such as Albertus Magnus, Roger Bacon, Vincent de Beauvais, Duns Scotus, Giordano Bruno, \&c.), but also a dissertation on the respective influence of the Platonic and Aristotelian philosophy, on the long controversy between the Nominalists and Realists, and on the men and manner of thinking of that age. He considers that the 'Imago Mundi' of Cardinal Alliaco, which Columbus carried with him on his voyage, had great influence on the mind of the illustrious navigator; and notices the curious fact, that the passage in this work which Columbus himself refers to as most deeply impressing him, is a transcription, word for word, made by the Cardinal from the Opus Majus of Roger Bacon! The wbole style of this discussion well illustrates those peculiarities of Humboldt's mind of which we have already spoken.

But our author rightly assigns an earlier date than that of Columbus to the first discovery of the American continent. Rejecting, as exploded, the tale of tribes speaking a Celtic dialect having been found on the coasts of Virginia, we are bound by very sufficient proof to admit that the coasts of Labrador and New England were known to the Icelanders and Norwegians, through their intervening settlements in Greenland, more than eight centuries ago; - that they partially settled in Vinland, as they called the country forming the coast of the New England States; - and that a bishop went on a Christian mission to the colonies thus established. These narratives, hitherto known and accredited by a few 
only, have of late years received ample confirmation from the researches of Rafn, the great northern scholar of our own times. The documents which he obtained and has published, attest not only the act of discovery, but indicate by the direction and length of voyage, by the times of sunrise and other curious particulars, the exact coasts thus examined, including Newfoundland, Nova-Scotia, Massachussets, \&c.* These various records, both numerous and authentic, come to us from that extraordinary island of Iceland, which during the 11th, 12th, and 13th centuries, created and maintained amidst its snows and volcanic fires, a literature that would have honoured the happiest climes of Europe. Succeeding the period thus signalised to us, a series of physical and social calamities extinguished this northern light; at which later time and in the same gloom, we lose sight of the land of Vinland, and all traces of this remarkable discovery disappear. Should we ever regain them, it must probably be on the American coast itself. But the ruder Norsemen left behind them no temples or palaces like those of Nimroud, to be disentombed for the admiration and instruction of distant ages; and their simply written records alone remain to attest this ancient discovery. $\uparrow$

We have already had occasion to notice the Introduction to the third volume of the Cosmos, when speaking of the entire work. This volume itself is occupied solely with the subject of Astronomy, under the title of ' Special Results in the Uranological portion of the Cosmos;' a cumbrous form of expression which well justifies the remarks we have made

* In a Dissertation on the History and Literature of Iceland, prefixed to Sir G. Mackenzie's Travels, I have stated my entire belief in the validity of this claim, founded on books and other documents I procured when travelling in that Island. But my information was small in amount compared with that which Danish antiquarians have since obtained on this curious subject.

+ Rafn, Antiquit. American. 
on the phraseology of Humboldt. As a treatise on the actual state of astronomy it is undoubtedly able and complete. Without entering upon demonstrations, it seizes all the salient points in this wonderful department of human science; and discusses in a spirit of high philosophy, both the results hitherto obtained, and the great problems remaining open for future research. So much, however, has been written on this subject of late years both for scientific and popular purposes, that we shall not follow our author formally through it; but merely make such comments as may occur to us on particular portions of the volume, and especially on those which relate to the progress of discovery among the Fixed Stars. This is the part of their vast domain in which astronomers have recently laboured with the greatest assiduity and success; availing themselves at once of the increased perfection of instruments, and of those improved methods which are best fitted to obviate all sources of error. The results obtained, and the inferences thence derived, are such as may well astonish even those familar with such studies. Following the order of the volume before us, we shall advert to the points which may especially illustrate the latest progress of these researches, and convey to our readers some idea of their boldness and grandeur.

The first chapter, 'On Cosmical Space,' brings us at once into this great field. After remarking that only separate parts of this space are accessible to measurement, our author adds, -

The results, which surpass all our powers of realisation, are brought together with complacency by those who take a childish pleasure in large numbers; and even imagine that, by means of images of physical magnitude creating astonishment, they peculiarly enhance the sublimity of astronomical studies.

If we understand this remark rightly, it rather surprises us; - in the first place, because Humboldt himself and the best astronomers constantly and needfully employ such illus- 
trations; and, further, because they do really in many cases convey to the mind larger and clearer conceptions of relative space. We admit at once that few of the distances expressed by astronomy are in strictness brought home to the understanding, even by those most familiar with such contemplations. One of the smallest celestial admeasurements, that of the Moon's distance from the Earth, can only be appreciated by bringing in other more common relations of comparison. And when we find that the star 61 Cygni is 592,000 times as distant as the Earth from the Sun, our reason, while satisfied of the certainty of the means by which this result is obtained, can raise no idea commensurate in any sort or kind with the vast array of numbers set before us. But we may aid ourselves in some degree by bringing in a new element-that of time - as a measure of space. We know from other sources that light is transmitted through space at the rate of more than eleven millions of miles in a minute. The distance of the Star just mentioned is such, that light proceeding from it, and travelling unceasingly at this rate, would require more than nine years to reach the earth! Now this new mode of measurement is as incomprehensible as the other, in a strict sense of the term; yet our conception is felt to be enlarged by its use; and new relations are perceived, even by those who look on the mere surface of the science.

Another case we will put; - because, amidst a like host of numbers, a practical conclusion is involved in which we of this nether world are not wholly unconcerned. With his wonted sagacity the elder Herschel obtained proof of what had been the prior suggestion of Bradley, that our Sun, with the whole attendant planetary system, has a proper continuous motion in space; of which motion he himself indicated the course and direction. With the methods employed in this great research we have no present concern; but may simply mention that time and the observations of later 
astronomers have fully confirmed the fact; and demonstrated the motion of the sun to be about equal to its own semidiameter, or what is nearly twice the distance of the moon from the earth, every single day. Whither is this vast and unceasing translation in space to lead us? or what collision or other consequeuce may it finally involve? Certain answer there can be none; though perhaps we may admit the idea of revolution round some centre of gravity - (visible or invisible, single or a system of bodies) - as more consistent with the analogies of nature than any other. But against any sudden catastrophe from this movement of our system in space, we are guaranteed by what we know of the distances of the fixed stars. The star we have already taken as an example, 61 Cygni, though not so near to us as $\boldsymbol{a}$ Centauri, and some others whose parallax has been obtained, is probably much nearer than the majority of those visible to the eye. Yet the Sun, moving at the rate we have just named, would take nearly 400,000 years to accomplish this measured distance! Here then again we gather knowledge and light from amidst this cloud of numbers. We cannot comprehend the whole, but we gain certainty as to a part; and the general conclusion is made more accessible to all.

In this chapter on 'Cosmical Space,' Humboldt takes up the enquiry as to the existence of an Ether or material medium, occupying and filling the great domain of the universe; but makes it rather a record of the opinions of others than any distinct expression of his own. It is in truth, in various ways, one of the most subtle questions which can exercise or perplex the human understanding. On one side it approaches the region of metaphysics;- everywhere it passes beyond the dominion of the senses, and of those exquisite instruments with which human ingenuity has aided them. Modern science thoroughly instructs us that matter and material organisation may exist, and molecular actions take 
place, demonstrable only in their effects; but through these various effects demonstrable with mathematical certainty. The sciences of Optics, of Chemistry, and of Electricity, furnish us with numerous examples of this nature, had we room to quote them. Apart, indeed, from all direct proof, we see no difficulty in admitting an ethereal medium (we use this term in default of a better) as occupying the regions of space. Without such admission, in fact, it is hard to conceive how the physical forces or actions which we know to be transmitted to us from other bodies in the universe around, should have existence, or be capable of this transmission. Whether gravity be a force per se - or, as some have sought to prove, a power residual upon the balance of other atomical attractions and repulsions - equally must we reason upon it as a material transmission of power, if we reason at all. The phenomena of light, on the emission theory, presume a luminous matter capable of pervading space from its uttermost depths. On the undulating theory, now generally admitted, they require and are thoroughly consistent with the notion of an ethereal medium capable of transmitting those waves, the relative magnitude, velocity, and interference of which produce all these wonderful results. Heat and light have close kindred in their physical conditions, and future research will probably render this association closer, in relation to a common cause. Though we have some observations from Bessel and Sir J. Herschel on the apparent subjection of the tails of certain comets to a polar force independent of gravitation, which may be electrical, we do not yet possess any absolute evidence of electricity under its magnetic or other forms being transmitted to us through space. Recent enquiry, however, has brought us closely upon this fact; and the intimate relation, by mutual convertibility, of all these great agents, makes it probable that time will disclose their common connection with some intervening medium among 
the worlds which surround us. Modern science is thoroughly awake to all these points of high philosophic enquiry.

While upon this topic we must briefly advert to the seemingly more direct evidence of an ether, derived from the diminishing periods of Encke's comet, and from the zodiacal light. Upon the latter proof we cannot at present place any certain reliance. But the observations on Encke's comet have high value from their uniformity of result; showing a tangential force acting constantly upon this body in its orbit, which can only well be explained by the supposition of its motion through a resisting medium. Here again time, and repeated observations, will probably give certainty to our future knowledge.

The second chapter, 'on Natural and Telescopic Vision, the Velocity of Light, and Photometry,' is associated with a valuable note by Arago, upon the effects of telescopic glasses on the visibility of the fixed stars. He conceives that high magnifying powers facilitate the finding of any star, not by sensibly enlarging its image, but by bringing to the eye a larger quantity of light, and at the same time contrasting it more strongly with the aërial field through which the star is seen; - the telescope magnifying, according to his view, the distance between the illuminated particles of air in the telescopic area surrounding the star, and thus giving a darker surface in contrast with the intense and concentrated light of the latter. There may perhaps be a little fancy in the latter part of this explanation; but we are not entitled to cavil at it, having before us the many extraordinary and complex phenomena of the visual power as directed, with or without aid, into these regions of space. We have always considered the original papers of Sir W. Herschel on this subject, the eloquent commentary upon them by Arago, ${ }^{*}$ and the labours of

* Analyse Historique et Critique de la Vie et des Travaux de Sir William Herschel. - Annuaire pour l'An 1842, par le Bureau des Longitudes;-a memoir 
Bessel, Struve, Sir J. Herschel, and Argelander, in prosecution of the same question, as among the loftiest efforts of astronomical science; and almost as striking in the methods pursued as in the results obtained. They define the power of that wonderful organ the human eye, over objects in a depth of distance which the human imagination strives vainly to reach; - they indicate the increase of power gained by artificial instruments of vision; - they show the relative depths in space at which luminous worlds are visible to us; - and they explain those irregularities which arise from the structure of the eye and imperfect methods of its use; from the faults of instruments; from atmospheric conditions; and, finally, from the properties of that marvellous element of light itself.*

It is easy to enumerate these particulars, but hard to convey an idea of the grandeur of the objects which are thus brought within the scope of human research, and of the speculations legitimately derived from them. When we are led to believe, on reasons scarcely admitting of refutation, that there are stars made visible to us, the light of which reaching our telescopes at any given moment, must have been emitted from these stars nearly 2,000 years ago, the result is one which no language can duly denote, and of which the simplest expression is the most sublime. Were it not for that infirmity of man's present state which is ever dragging him downwards to the things of the earth, it might seem impossible for the astronomer, who has lived and laboured

which any one wishing to gain knowledge of these remarkable researches, will do well to study throughout.

* We may mention as an example of the singular and unexpected results obtained by these researches, the discovery of Struve that a telescope admitting no more light than the simple pupil of the eye, does actually show nearly twice as many stars in a given space as are seen by the eye alone. This curious fact renders necessary a correction of Sir W. Herschel's view of the proportionate distance of stars visible to the naked eye, and telescopic stars. 
amidst these high objects, to submit himself to the common coil of worldly affairs. An eminent name is present to our memory when we make this remark. While lamenting, as all must do, the recent loss of Arago, we may express our belief that he himself found deep cause to regret that change, which removed him for a time from the scientific labours of the Observatory and Institute to the revolutionary government of his country. This avowal indeed we have ourselves more than once heard him to make.

The ensuing chapter ' on the Number, Distribution, and Colour of the Stars, and on the Milky Way,' has additional value in some numerical results of great exactness, furnished to Humboldt by that eminent astronomer Argelander of Bonn. From various combinations of the data afforded by star-catalogues, he obtains as a mean number from 5,000 to 5,800 stars visible to the naked eye throughout the entire heavens; while, carrying the list forwards to telescopic stars of the ninth magnitude, he finds a total result in round numbers of 200,000 stars! And here again we come upon one of those curious relations so frequent in astronomy, which transcend all common ealculation. The imagination, unaided by science, might well conceive that-this host of numbers would crowd and cover every point in the sky; and would hardly lend belief to the assertion that each of these 200,000 stars, if equally distributed, would occupy to itself an area almost equal to that of the full moon. Yet so it is; the fact being very precisely determined that 195,290 surfaces of the moon, in its mean diameter, would be required to cover the whole heavens. This relation is of course a mere accident; the stars being very unequally distributed, and their classification by apparent magnitudes an artificial one. But there is value in the illustration it affords; and legitimate pleasure as well as instruction in the results which these ponderous numbers thus place before us. 
It becomes more difficult to obtain understanding or assent to the far higher numbers and relations which lie beyond. The stars of our sidereal system, down to the 9 th magnitude, form but a small fraction of those which the space-penetrating powers of the telescope, in what have been very appropriately called star-gaugings, now render visible to the eye. Sir W. Herschel calculates that 18 millions may be seen in the Milky Way alone. Struve estimates for the whole heavens 20,374,000 stars. Allowing a large margin for these numbers, which can be but approximate, they yet possess certainty enough from the methods of observation employed, to impress upon the mind the immensity of this universe of worlds. It may be that the feelings receive more of this impression than the understanding. But there is one important fact which the intellect can scan, and which even in its simplicity has a grandeur commensurate to the magnitude of the objects concerned. Whatever be the actual nature of the two great physical powers, Gravitation and Light, we have absolute proof that these pervade and operate throughout the whole of the vast system thus disclosed to us. Gravitation acts by the same law among the double stars, as in the fall of an apple, or the flight of a stone on the earth. The solar spectrum on a screen, and the ray polarised by a crystal, represent properties of Light, which we have every reason to believe identical with those of the same element, as transmitted to us from stars the most remote in space. Here then we attain at once the proof of the unity of power, of design, and even of instrumental operation, in the creation of the universe. It is an argument as clear and cogent as any that we habitually employ in the ordinary transactions of life; - the marvel being that we, the feeble and shortlived tenants of a mere satellite in this system of suns, should reach by any road to these high conclusions which everywhere border on infinity. 
The labours of astronomers have of late been sedulously and worthily directed to the formation of catalogues and maps of stars; in which their places are fixed with accuracy enough to permit the recognition of any changes occurring amidst them. The phenomena of proper motion, and of the appearance of new stars or the disappearance of old ones all objects of great interest to the science-have derived much illustration from the labours thus directed. These Star-Maps have fulfilled another important purpose in aiding the discovery of planetary bodies belonging to our own system. Of the numerous planetoids now discovered between Mars and Jupiter, the greater number may be considered as due to this method of assisting and correcting observation. We have a more illustrious example to the same effect in the circumstances of the discovery of Neptune; which we believe to have been aided by a particular sheet of the Star-Maps of the Berlin Academy, published only a few days before Galle directed his telescope in search for the predicted planet. This great Prussian work, representing the stars to the 9th magnitude inclusive and many of the 10th, in a cycle of $15^{\circ}$ on each side of the equator, is now approaching its completion. The number of stars included in it will fall little short of 200,000. Bessel, Harding, Argelander, and others have laboured in the same vast field; and the zone to which Argelander has extended his observations has afforded him already a list of more than 100,000 stars. Our own countryman, Mr. F. Baily, devoted the latter years of his valuable life to the British Association Catalogue, founded on those of Lalande and Lacaille; and the Royal Observatory at Greenwich has largely contributed to the same department of astronomy. It is impossible to appreciate too highly the scientific value of these labours. Had we possessed catalogues equally complete of the time of Hipparchus, numerous facts in sidereal astronomy would probably have 
been known to us which it may now take centuries to disclose.

After describing the distribution of the stars and the Milky Way, the great trunk of our sidereal system, M. Humboldt. proceeds to treat of the newly appearing and vanishing stars; and of those which exhibit variations, periodical or otherwise, in brilliancy or colour of light. This again is a part of astronomy fertile for both reason and imagination to work in. It records great mutations in the remote worlds of the universe - the causes known to us by hypothesis only.* The new star seen in the time of Hipparchus led him to begin his catalogue, and suggested to Pliny the question Stelloe an obirent, nascerenturve? - an enquiry still unresolved, and which probably may ever remain so. Appearance or disappearance do not needfully imply creation or extinction. Light is the sole medium through which we have intimation of these events; and we know, by the instances of the planets of our own system, that the celestial masses are not necessarily self-luminous, and may be rendered so by reflection only. But the suddenness of the event in certain of these cases shows undoubtedly some mighty acts of change, which we can contemplate only in their results. The fact long noted, that a large proportion, if not all, of the new stars observed have appeared in or near the Milky Way, has done more to excite than aid conjecture; and we must not stop to relate the speculations which have been hazarded on the subject, as none of them have any higher sanction than that of possibility. They are however in some degree justified by the limited number of contingencies open to us, and

* That excellent observer, Mr. Cooper, working in his private Observatory near Sligo, and preparing a catalogue of 60,000 stars near the ecliptic, found that 77 stars, peculiarly noted, had disappeared, nearly 50 of which had come under his own prior observation. The planetoids between Mars and Jupiter will probably account for some of these disappearances, but certainly not for all. 
by the eventual verification of other conjectures in astronomy, which at first appeared almost as far beyond the scope of human research.*

Though it may seem rash to associate an anomaly in our own planetary system with these changes in remote sidereal space, we cannot forbear noticing again the wonderful group of small planets between Mars and Jupiter ; - the sole instance in our system, with the exception of comets, where it becomes probable that some sudden catastrophe has occurred, changing essentially the condition of a great body revolving round the Sun. We venture to use this word of catastrophe, because we can hardly refuse belief to Olbers's conjecture of the disruption of a planet in this region, - seeing the great number of these small revolving bodies located in the same part of planetary space; their highly inclined, excentric, and intersecting orbits; and other peculiarities, which render them wholly anomalous in the system to which they belong. $\uparrow$ Disruption implies the action of a given force, either from without, or from within the mass disrupted. We have no knowledge of any external agent (for comets would seem out of the question) capable of effecting this wondrous disseverment. If we might risk conjecture on a point thus obscure

* We believe the latest new star observed to be that discovered by Mr. Hind, in April, 1848, at the Observatory in the Regent's Park - a place which this admirable observer has already rendered eminent in the annals of astronomy. When discorered the star was of the 5 th magnitude, but progressively lost its lustre; in 1850 was only of the 11th magnitude; and has now, we believe, disappeared altogether. That extraordinary Chinese document, the Ma-tuan-lin, to which we have recently alluded in an article on Aerolites, contains many valuable notices of these new or temporary stars.

$\uparrow$ The first of these planetoids, Ceres, was discovered in 1801. Before 1807 three more were found; and these four only were known when the first rolume of the Cosmos was published. The fresh race of discorery was begun by Encke in 1845. In his third volume (1851) Humboldt records fourteen then known. In 1854, the number had reached twenty-eight. Since that time more than forty have been added to the number. The systematic search for these singular bodies, begun in 1846 by Mr. Hind; has largely contributed to these discoveries. 
we should incline to the theory of disruption and unequal projection of the fragments into space, from some force within the body, acting when its primitive consolidation was yet incomplete; - such force as, on a smaller scale, we must presume to have been concerned in raising the lofty mountains and forming the enormous craters which characterise the surface of the moon. Or might we enlarge the hypothesis and suppose it possible that the vast neighbouring mass of Jupiter, already consolidated, aided the action of internal forces in bringing about the anomaly in question? * We pause here however; not solely from the uncertain ground on which we are treading, but because this instance was brought forwards merely as a possible illustration of the greater changes in bodies far more remote from us.

Closely related to the new and vanishing stars are those of variable brightness; of which our author treats at considerable length, and with the advantage of a very valuable communication from Argelander on the stars of periodical variation. $\dagger$ With our limited space we can refer to a few only of the facts which have been accumulated on this curious subject. The phenomenon of variability is more frequent in red stars than in white ones. It exists apparently in stars of every magnitude. The total number of variable stars, with determinate periods now known, is about 24; but many others will doubtless be added by future observation. The periods of

* The celebrated Kant, in his 'Natur-Geschichte des Himmels,' adopts the idea that the smallness of Mars depends on the prodigious attraction of the mass of Jupiter, acting when the planets were in course of formation.

+ We learn with great satisfaction that Argelander is composing a treatise on this very interesting branch of astronomy. It cannot possibly be in better hands. As an instance of his zeal in research, we may mention that he has succeeded in tabulating above 100 observations on Algol, including a period of fifty-eight years, during which there must have occurred not fewer than 7600 periods of variation of this remarkable star; each marked by equal times of decreasing and increasing brightness, with a stationary interval of nearly three days between. 
variation differ as much as in the ratio of 1 to 250 . The period of $\beta$ Persei, about 69 hours, is the shortest; - one of 495 days the longest yet ascertained. In some stars the periods of increasing and decreasing brightness are equal; in several the light increases more rapidly than it diminishes. In certain stars (as Algol, Mira Ceti, and $\beta$ Lyræ) the periods themselves undergo a periodical variation; and the lastnamed star is remarkable from having a double maximum and minimum in each of its periods of 13 days. Among the variable stars are some very familiar to us, as the Polar Star and two or three of the Great Bear. One of the most splendid examples yet observed is that of $\eta$ Argus in the southern hemisphere, described by Sir John Herschel in his Observations at the Cape of Good Hope. The peculiarity and grandeur of the changes in this extraordinary star - raising it, though at irregular intervals, from the fourth magnitude to the vivid brightness of Sirius or Canopus - are recorded by a pen well capable of such delineation.

The facts thus briefly cited are prolific of speculations of the same character as those applied to the new stars, and equally incapable of present solution. The general inference of rotation on an axis or revolution about a centre, rendered probable from other sources of evidence, is very directly suggested to the mind, especially in the case of the stars of periodical variation. The phenomena themselves, though far more remarkable in variety and degree, are not wholly without analogy to the conditions of our own central luminary. The solar spots, as more accurately observed by modern astronomers, indicate changes in the state of the Sun - or of the luminous envelope surrounding it - which visibly affect the amount of light emitted, and might alter more or less its brilliancy, if seen from remote distances as a star. But we cannot carry the argument beyond a bare suggestion, for we are hitherto ignorant of the cause of these 
changes in the Sun; and can as little explain that singular phenomenon of periods of temporary darkness or obscuration, independent of solar eclipse, of which we have authentic notices from various ages and parts of the world. Upon the latter fact, however, we do not dwell in the way of analogy, as it may more probably be due to atmospheric or meteorological causes than to actual changes in the Sun itself, or possibly to the passing intervention of clouds of meteoric matter between the Sun and the earth, during some part of the annual revolution of the latter.

We must not omit to notice here the fact, that several of the more conspicuous fixed stars - Sirius notably among the rest - exhibit dark lines in their spectra, permanently the same for each; and strictly analogous to those of the Sun, though differing in distribution. There is a deep interest in this phenomenon. It brings into direct association some of the great physical laws and powers which act in our own system, with those of worlds infinitely remote. We may not venture to infer any such identity of matter, as the spectrum analysis has made known to us in the Sun. But that the material of these worlds is analogous in kind, this principle of research fully justifies us in believing.

Seductive as are these speculations, we must hurry on to other topics not less so. The next in order how.ever - that of the Double and Multiple Stars - is fast passing from the region of speculation into that of exact science; and the great law of gravitation is becoming to our knowledge absolutely coextensive with the existence of matter in motion, at whatsoever distance in space. That mere points of light - many of them only visible by the telescope, or from their vast distance seem as single though really including two or more stars - should be made to yield the same conclusions as the planets and satellites of our own system, is a marvellous instance of human prowess; and scarcely credible to 
those who have not familiarised themselves with the methods, as well as results, of this high attainment. The researches on the Double Stars, begun by Mayer in 1778, and since prosecuted with such admirable zeal and success by the two Herschels, Struve, and other astronomers of our own day, furnish a present record of at least 6,000 multiple stars; of which number about one-third were discovered by Sir J. Herschel in the southern hemisphere. Of these very many are doubtless only optically double - that is, nearly in the same line of visual direction, but at very different distances. and having no actual relation to each other. But about 700 have been shown to undergo such changes of relative position as to prove their physical connection in revolution; and binary star-systems are now recognised, not merely by proximity and by these changes, but also in many cases by actual computation of the elements of the orbits described by one or other of the connected stars. This computation, showing in some instances remarkable excentricities of orbit and long periods of revolution, has now been extended to about sixteen double stars. One of these, $\zeta$ Herculis, has already twice completed its circuit of thirty years under observation, and presented the actual phenomenon of the occultation of one fixed star by another - an eclipse as absolute as any of those of which we keep record in our own system. The calculation of two particular periods of revolution of double stars at more than 500 or 600 years respectively, may give some idea of the scale by which are measured these remote movements in space; and we cannot better illustrate the grandeur and completeness of the research than by stating that Bessel, having determined the distance of 61 Cygni, a double star, was able from this and from the orbital motions already ascertained, to deduce the mass and weight of the two stars thus connected by mutual attraction.*

* Eren since the first edition of this volume was published, a remarkable 
We can but advert, in passing, to the curious observations of Struve, Arago, and Herschel, on the contrasted and complementary colours of many of the double stars, and must follow our author hastily through the further questions of the distance of the fixed stars; - of the proper motion of our own Sun and other stars in space; - and of the existence of a common centre of gravity and revolution for the whole sidereal system, to which our Solar system belongs. To some of these topics we have already had occasion to allude in the way of illustration. The determination of the distance of certain of the fixed stars is one of the achievements of late years. It fulfills a desire of much longer date, which had been rendered unavailing by imperfection of instruments, the difficulty of separating the parallactic and proper motions of stars, and other causes. Abstractedly, the problem of finding the parallax is one of simple trigonometry, and astronomers had already provided the measure of the diameter of the earth's orbit as a base for the operation. Yet even this vast base, of nearly 200 millions of miles, failed to render any assured angle of parallax to the earlier instruments employed in the attempt. And it was not until the workshop of Munich had furnished its admirable refractors, and micrometers been added to them capable of designating the 60,000 th part of an inch, that the great result was unequivocally obtained. After three years of patient observations, begun in 1837, Bessel announced the discovery of the parallax of 61 Cygni; and the wonderful conclusion as to distance founded upon it, to which we have before referred. The certainty of the fact was fully attested by the exact correspondence of the annual changes in the place of the star; the parallactic variaaddition has been made to our catalogue of double stars, in the discovery of a companion to the splendid star of Sirius. This had before been conjectured by Bessel, from his notice of a periodical variation in the right ascension of Sirius; but the discovery was finally made by Mr. Clarke of the Harrard Observatory, Massachusets. 
tion increasing and diminishing precisely as ought to happen in relation to the annual motion of the earth in its orbit. The variation, thus certain in proof, was so small in itself as to be measured by an angle of scarcely more than one-third of a second - a striking example of what is common in every part of astronomy, the attainment of results sublime in their magnitude by methods of the most exquisite minuteness and refinement. Even in this very minuteness of means there is something of grandeur to the thought, seeing what are the objects thus attained. He must be a man of obtuse mind who can regard with indifference the slender lines of spiderweb intersecting the field of the telescope, to mark the exact moment when the star passes its axis; - or listen without emotion, in the otherwise silent Observatory, to the measured beats of the clock which records with unerring truth these moments of transit.*

Other astronomers were at work at the same time, and with the same object as Bessel; and the labours of the last fifteen years, combined and compared with those of Struve of an earlier date, have given the parallax and distance of upwards of thirty Stars; - not with equal certainty in every case, but continually approximating to it. Henderson and Maclear obtained a parallax of nearly one second for a Centauri, the finest double star of the southern hemisphere, thus placing it three times nearer to us than 61 Cygni; while $a$ Lyræ, another bright star, yielded to Struve a parallax of little more than a quarter of a second, indicating thereby a distance of 771,400 times that of the earth from the Sun, and a time of twelve

* We may mention here that Mr. Bond, by a happy adaptation of one science to another, has applied an electrical apparatus of admirable construction to the still more instantaneous and perfect registration of astronomical events.

Another eminent American astronomer, Mr. Mitchel of the Albany Observatory, has given us what he terms a personal equation for astronomical observers, founded on the different capacity of different persons for receiving and recording instantaneous impressions on the eye. 
years for the transmission of light to us. The most diligent and successful observer in this part of astronomy, M. Peters, has carried the determination of parallax in some cases even to the tenth of a second; - thus indicating distances 'which we may well hesitate to translate into numbers either for space or time.* Such research is likely to be carried yet farther, but the main results are probably now ascertained. We may name as one of these results, the proof of the great difference in the magnitude of stars, derived from the want of any proportion between their degree of brightness and their parallax. This difference might have been supposed probable, but it is thus rendered almost certain.

The actual magnitude, however, of any of the Stars is a problem hitherto insuperable; and probably destined ever to remain so, seeing that the best telescopes do not give to them any real disk or angular diameter. The only approach to a solution is by comparative photometrical observations on the light of our own Sun, and of certain conspicuous stars;a method open to various sources of error in its progress, and rendered doubtful in its results by our ignorance of the relative intensity of light emitted from these different bodies. The intrinsic brightness of $\boldsymbol{a}$ Centauri has been estimated at $2 \frac{1}{2}$ times, that of Sirius even at 63 times the light of the Sun; yet we are not entitled to draw thence any direct conclusion as to the comparative magnitude of these great globes. The only inference attainable is that stated above, of great diversity of size among them, corresponding in this respect to the conditions of our own planetary system. We do not

* Mr. Peters's observations are recorded in Strure's 'Etudes d'Astronomie Stellaire'-a work remarkable among all others of our time for its profound views in this department of the science. Had we room for it, we might give, what we do not find in Baron Humboldt's volume, an account of the refined method of investigation by which M. Struve obtains, first, the relative mean distance of the stars classed under different magnitudes; and then, by reference to the distances actually determined, the absolute mean distance of each of these classes of stars. 
find in the volume before us any explicit notice of this question; though much that is valuable in the account of the photometrical researches recently applied to the stars.

The discovery of the translation of our own Solar system in space, and of the absolute motions of the fixed stars (as they have been termed), is another of the achievements of modern astronomy. At this time, indeed, no proof exists that any body in the universe is stationary in the strict sense of the word. All present evidence tends rather to establish the universality of motion, wherever there is matter in state of aggregation; or perhaps we may say, matter in any state. We have elsewhere, for the purpose of illustration, spoken of that great and continuous movement of our own Sun (fully proved by the observations of astronomers), which is carrying it in the direction of a point in the constellation Hercules, at the rate of more than 140 millions of miles every year. The absolute motion ascertained of several other stars (independently of the orbital revolutions of the double stars, and with deduction of all that belongs to the precession of the equinoxes, the nutation of the earth's axis, the aberration of light, and parallax) attests a great fact in the economy of creation, which one can scarcely regard without a certain feeling of awe, associated as it is with evidence thus wonderful of the number, magnitude, and remoteness of these surrounding worlds. The amount of annual motion now measured in different stars varies from 1-20th of a second to nearly 8 seconds; and without any relation between the actual amount and the brightness of the star. In the 2,000 years elapsed since the time of Hipparchus, the proper motion of Arcturus must have altered the position of this star in the heavens $2 \frac{1}{2}$ diameters of the Moon; while one of the stars in the Swan must have moved over a space of 6 diameters in the same period. In this part of astronomy especially, time cannot fail to confirm and extend the facts 
ascertained, and to enlarge the conclusions derived from them.

While treating all these topics with his wonted ability and care, we think that Humboldt somewhat too hastily passes over the question of a common centre of gravity and revolution of the sidereal system; seeing those vast labours of Mädler in the Observatory of Dorpat, which, even if not admitted to substantiate his opinion, do yet form one of the eras in sidereal astronomy, and a basis for all future enquiry. We cannot but admire the skillful approximations by which he narrowed the field of research for this great centre: limiting it first to the constellation Taurus; and finally, by testing the proper motions of each star in this region, locating it in the group of the Pleiades and in the star Alcyone, the centre of this group. Of the fourteen stars which the telescope shows to be clustered round Alcyone, all have their proper motions in the same direction and nearly of the same amount; and extending this remark to upwards of one hundred stars within $15^{\circ}$ of this centre, Mädler found that all which had any certain proper motion moved in such exact conformity with his hypothesis, that he declared he would abandon it if one star could be found within $25^{\circ}$ of the presumed centre, with a well-ascertained motion in an opposite direction. If we durst allow anything of romance to blend itself with pure astronomy, this theory, which places amidst the Pleiades the centre of gravity of the universe of stars composing our system, might well lay hold on the imagination. It awakens the memory of the many passages of poetry of every age by which this beautiful group has been in some sort endeared to us. It recalls to mind the classic story of the lost Pleiad. In the aspect, too, of these stars there is much to engage the fancy. They are seen, in the midst of an almost starless space, a close and brilliant cluster - inviting the eye to number them, yet by their compression 
making it barely possible to do so. Nearly a century ago an old English astronomer, Mitchell, computed the chances as more than half a million to one, that the stars of the Pleiades could not have been thus arranged by accident; and the computation has been sanctioned by later authority. They are a system in themselves, and in their concentration and conceivable magnitude may possibly form a powerful centre of attraction to worlds around them.

While saying thus much of Mädler's theory, from the slight notice of it in the Cosmos, we are bound to add that a serious objection exists in the fact that the Pleiades lie $26^{\circ}$ out of the plane of the Milky Way; and that it is scarcely possible dynamically to suppose any general movement of rotation out of the plane of this great stratum of our stellar system. To settle the arduous question, whether a rotation of the Galaxy in its own plane exists or not, Sir .T. Herschel proposes the assiduous observation in right ascension and polar distance of a certain number of stars in the Milky Way, judiciously selected in both hemispheres, and including all magnitudes down to the lowest distinctly observable; and he asserts his belief that a strict perseverance in such research for thirty or forty years could not fail to settle the question. It is an object well worthy of the labour thus suggested.*

The Chapter on the Nebulæ has all the interest which belongs to a masterly outline of the most wonderful department of human research. All the numerical measures of space and time with which we have hitherto been dealing, dwindle into nothing when compared with those which the nebulæ place before us. Instead of numbering the stars of a system, we are here numbering separate systems of stars. The nebulæ, whose places in the heavens have been exactly determined, now surpass 3,600 ; exceedingly various in outline,

* Outlines of Astronomy, p. 589. 
superficial extent, and intensity of light, but from their distance rendering it uncertain what are their true relations in these respects. This distance is one strictly immeasurable. The calculated distances of certain of the fixed stars of which we have already spoken, enormous though these are, scarcely furnish a unit for the comparison. Approximations, indeed, have been made, but by methods which it would require more space than we can give to render intelligible. Without expatiating then on this point, of which neither language nor figures can convey any true conception, we may state generally that the observation of the nebulæ is every year affording facts and problems of higher interest. Here are separate systems of worlds, numerous as above described, and each comprising probably as many as our own vast system of Suns. We have the common element of light, through which alone, indeed, we know of their existence. Observation has disclosed to us the most singular varieties - not merely in the visible extent of these nebulæ, which simple difference of distance might produce - but also in their configuration and manner of condensation around centres ; implying forces of attraction which, in default of knowledge from observation, we may reasonably from analogy suppose to be the same as those governing our own planetary system. Other direct means of knowledge regarding them we do not yet possess. But time (if it be still sufficiently allotted to the generations of man on the earth) and continuous observation by instruments of large and well-defined power, may give us somewhat nearer access to the physical history of these remote parts of the Universe. Every record of change here - whether it be concentration, diffusion, division, or disappearance - is a fact gained to science.*

* February, 1862. Even at the moment of sending this sheet to the press, I see a very interesting letter from Mr. Hind, relating, on the authority of several eminent observers, the total disappearance of a small nebula, in the constella- 
We have spoken of telescopes of large power, because such are essential to nebular astronomy. The great Reflector, for which science is so deeply indebted to Lord Rosse, has by its assiduous direction to the nebulæ, afforded three results, each showing the value of the vast telescopic power thus obtained. The first of these is the more correct knowledge of the true form and aspects of these wonderful aggregations of stars; a result well attested by the remarkable differences of certain nebulæ as seen through the telescope of six feet aperture, or through one of three feet only. The second discovery due to this high power is the extraordinary tendency to a spiral arrangement in these nebular systems; so frequent and so distinctly developed, that it is impossible to attribute it to accident alone. When the volume of the Cosmos now before us was published, only one or two instances of this phenomenon were recognised. They have since been multiplied in the same ratio with the multiplicity and minuteness of observation; and the results make it needful to suppose a common physical cause for this remarkable effect. The exact and beautiful drawings of these spiral nebulæ, which we owe to Lord Rosse's observatory, scarcely leave a doubt that some general law of aggregation and distribution has more or less governed them all. We are compelled however to rest here; for neither reason nor analogy gives us any knowledge of forces capable of fulfilling these physical conditions. If the attraction of gravitation be still the main element of power, as we have ventured to suppose, it must

tion Taurus, which he himself had discovered in 1852. A minute star, variable from the tenth to twelfth magnitude, almost touched the nebula, and served to authenticate its position and disappearance. Is this disappearance an obscuration from intervening non-luminous matter in motion? Or is it a change in the luminous or other conditions of the nebula itself? These are examples of the class of questions which such phenomena press upon us. Even unsolved, they bear testimony to the sublimity of the science, which can place these problems before the mind. 
act under circumstances or in connection with other forces, which control or otherwise modify its effects. But in pausing of necessity at this point, how sublime is the resting-place attained, and how far above the objects and contemplations which beset us in the ordinary course of human life!

The third great result derived from Lord Rosse's telescope, viz. the resolution into stars of many nebulæ before unresolved, bears closely on the question, so much agitated of late, as to the existence of a self-luminous nebular matter diffused in different parts of space, and forming the material out of which worlds are aggregated and systems of stars brought into being. This theory, sanctioned by eminent names, and plausible at least in its application to our own planetary system, found support in the aspect of such unresolved nebulous lights in the remote heavens. The simple fact that progressive increase of telescopic power has in the same ratio disclosed to us these luminous masses as clusters of innumerable stars, must be considered a cogent, though not decisive, argument agàinst it; the nebulæ still not analysed presenting the same aspect as those which have been recently thus resolved; and, perhaps awaiting only a higher power given to the eye, to afford the same results. Furthermore, it may reasonably be doubted whether mere nebulous matter, yet uncondensed into stars, could from distances such as these radiate light apparently equal in intensity to that of nebulæ known to be entirely composed of stars. The whole question, by the very terms of it, will be felt as one incapable at present of any complete solution. But the negative upon the modern nebular theory has been strengthened; and those bold speculations placed in abeyance which dealt with the consolidation of worlds as if it were matter of familiar observation, and within the compass and calculation of ordinary science. We acknowledge ourselves of the number of those who think this to be a salutary check, 
and in accordance with the true interests and legitimate course of physical enquiry.

Our author discusses these subjects with his wonted ability; and also the collateral questions as to the existence of nonluminous bodies in space (an opinion confidently held by several eminent astronomers); and the possible, or probable, loss of light in a certain ratio to the length of line it traverses through the heavens, as inferred by Struve from some of his recent researches.* These loftier but less certain speculations of the Stellar Astronomy are followed by a series of chapters on our own Solar system; including the Sun, the planets and their satellites, comets, the ring of zodiacal light, and meteoric asteroids. This part of Humboldt's work is admirably executed; lucid in arrangement, ample in details, and suggestive throughout of those great relations and inductions which form the true philosophy of every science. The deficiencies are such as belong chiefly to the date of publication, recent though this is. The number of the small planets recognised between Mars and Jupiter has been nearly quadrupled since Humboldt's first record of them. A third or inner ring of Saturn has lately been discovered; while the conjoint researches of Struve and Bond (the latter an observer of whom America may justly be proud) give some reason to believe that the whole annular system of Saturn has, since the time of Huyghens, been approaching nearer to the body of the planet, and cannot therefore be considered in the state - of stable equilibrium which Laplace had supposed. The two new satellites of Uranus, discovered by Lassell in October, 1851, were unknown when this volume of the Cosmos was printed, but are noticed in an appendix to it. In the Chapter

* Études d'Astronomie Stellaire, 1847 ; - in which work all these profound questions find their place. Struve entertains the belief that some of the anomalies of present calculation could be reconciled by supposing a loss of one per cent. of light, in passing through a distance, equal to that of an average star of the first magnitude; thus modifying in a certain degree the law of diminution in the inverse ratio of distance. 
on Comets there is a full account of the extraordinary phenomenon witnessed in January, 1846; - the separation, or splitting, of Biela's comet into two distinct bodies assuming different lines of movement. Here Humboldt expresses the anxiety, common to all. astronomers, for the evidence derivable from the next return of these twin comets within our sphere of observation. This return took place, as calculated, in the autumn of 1852 ; - the two nuclei were re-discovered, one of them three weeks after the other;-much farther separated in space, and affording a strong presumption that these two bodies are detached from one another for ever. The phenomenon, as regards our knowledge, is unique and not reducible to any ascertained law; though, perhaps, not wholly without relation to some of the aspects and changes noted in certain other comets of our own time.

We scarcely know whether to be satisfied or not with our author's account of Mr. Adams's participation in the discovery of the planet Neptune. The passages alluding to it, both in the text and notes, have obviously been carefully studied in the phrases employed; yet hardly give an adequate explanation of the peculiar circumstances of this great astronomical event. We quote from the text a few lines on the subject.

I think it right to forbear in this work from more than an allusion to the certainly earlier, but unpublished labours - not, therefore, crowned by recognised success_ of the highly distinguished and acute English geometrician, Adams, of St. John's College, Cambridge. The historical facts relating to these labours, and to Leverrier's and Galle's happy discovery of the new planet, are related circumstantially, impartially, and from well-assured sources of authority, in two Memoirs, by the Astronomer-Royal, Airy, and by Bernhard von Lindenau. Intellectual labours, directed almost at the same time to the same great object, offer, besides the spectacle of a competition honourable to both competitors, an interest the more vivid because the selection of the processes employed testifies the brilliant state of the higher mathematical knowledge at the present epoch. 
We ourselves admit fully the difficulty of the case; but we are very solicitous that Mr. Adams's merits in the discovery should not, from any accidents as to time or public communication, be underrated either by the present generation or by posterity; recollecting especially the circumstance, unnoticed by Baron Humboldt, that the planet was first seen (though not at the time recognised as such) through a telescope directed by Mr. Adams's suggestion to that point in the heavens, which his calculations indicated as the place of the disturbing body.*

We do not find in Humboldt's account of this wonderful discovery any notice of the singular differences between the assumed elements of the orbit of Neptune, on which Leverrier and Adams founded their successful calculations as to its place; and the actual elements as derived from present observation, and from comparison with its former position, when seen, without recognition of its planetary character, by Lalande fifty-eight years ago. The detection of these discordances is mainly due to the American astronomers, Walker and Pierce; and they have led the latter to affirm that the planet Neptune cannot really be that indicated by the calculations of Leverrier and Adams! - a conclusion much too strange and startling to admit of easy acquiescence. Sir J. Herschel, in his 'Outlines of Astronomy,' has happily elucidated the difficulty, and explained the error of this conclusion, by showing that the exact accuracy of the assumed or predicted elements was by no means necessary to the successful calculation of the place of the planet. Some points still remain open for solution; but they are such as future observations cannot fail to determine; and meanwhile all that is

* Without wishing to raise any question of relative merits, M. Leverrier's high reputation will admit of our stating, that the value which Mr. Adams affixed to the limits of the inferior axis of the presumed planet was considerably nearer the reality than that assigned by his competitor in this remarkable discovery. 
most essential in the question may be regarded as finally settled. The whole history of this discovery forms, beyond doubt, one of the most remarkable passages in the records of astronomy.*

We must advert here - though slightly, as befits what is not yet proved - to those refined observations of Leverrier on certain irregularities in the motion of Mercury, which led him to suppose another planet, still nearer to the Sun's body. To this he has already given a name, - an anticipation hardly sanctioned by the rough notices of Lescarbault, who is hitherto the only authority for its transits over the Sun.

In closing this article, which we have sought to render a just and impartial review of the volumes before us, we may add that there is reason to expect the publication of the last portion of the Cosmos in the course of the next few months. We would express our hope that it may be presented to the English reader under the same auspices as the volumes already published; where all that is more purely scientific bears evidences of that clearness and accuracy which General Sabine's superintendence was sure to afford; while the translator has done ample justice to the peculiar and striking phraseology of the original.

* I may, perhaps, be excused for mentioning here that, by a happy accident, I was with Encke and Galle in the Observatory at Berlin on the night of October 2nd, 1846, awaiting the dispersion of clouds for a sight of the new planet, when a letter was brought in from Leverrier, to whom the question of its name had been referred. Encke himself had selected the name of Janus; but the moment that Leverrier's letter put before him that of Neptune, he cried out, So lass den Namen Neptune sein! In the darkness of the Observatory a single lamp gave reading to the letter, which thus conveyed a name to all posterity! The spot and scene I cannot easily forget. 


\section{AUSTRALIA - CORAL REEFS.*}

[Quarterly Review, June 1847.]

TWHE volumes we have placed at the head of this article 1 narrate, or are connected with, those expeditions of maritime survey in distant regions of the globe, by which the credit and interests of England, as the great maritime and colonial power of the world, are maintained and enlarged. What we have hitherto accomplished of such research, though perhaps adequate to, does not exceed the demand that may fairly be made from a nation so circumstanced as to territory, commerce, and the arts and improvements of social life. In this matter there is an obligation due to ourselves, to other nations, and to posterity; and while deprecating, as we do, all narrow and parsimonious views in dealing with an obligation thus incurred, we may add our belief that no public expenditure can be more profitably made - no public services more

* 1. Narrative of the Surveying Voyage of H.M.S. ' Fly', commanded by Captain F. P. Blackwood, R.N., in Torres Strait, New Guinea, and other Islands of the Eastern Archipelago, during the Years 1842-1846, together with an Excursion into the Interior of the Eastern part of Java. By J. Beeta Jukes, M.A., F.G.S., Naturalist to the Expedition. 2 vols. 8vo. 1847.

2. Structure and Distribution of Coral Reefs, being the first part of the Geology of the Voyage of the 'Beagle,' under the command of Captain Fitzroy, during the Years 1832-1836. By Charles Darwin, M.A., F.R.S., F.G.S. London, 1842.

In this article there occurs some commingling of dates, such as I have alluded to in the preface. That part of it which relates more especially to Australia has been added to and corrected, from a later article in the 'Edinburgh Review, upon the Geographical Researches of our own time. 
beneficially applied - than in forwarding those large researches and surveys by which, while nurturing officers and seamen of the highest class, we open new channels and give fresh vigour and greater security to the undertakings of commerce over the globe.

In this age, indeed, we can no longer send adventurers forth to achieve the discovery of new lands, or shores vaguely shadowed out by the imagination of antiquity. With the exception of the ice-bound tracts which circumscribe the poles, and into which the disciplined boldness of our navigators has of late deeply penetrated - and with the further exception of those large islands which form the south-eastern boundary of the Indian Archipelago - all the great outlines of the globe may be said to have been drawn and defined. No Atlantis now remains to be sought for in the Western Ocean; nor is there space or spot anywhere left for those romantic wonders of the traveller, so pleasantly pictured by Ariosto:-

Che narrandole poi non se gli crede, $\mathrm{E}$ stimato bugiardo ne rimane.

The human tails of Lord Monboddo's theory are no longer considered hopeful subjects for research; and even if the modern story of a tribe of pigmies to the south of Abyssinia were better accredited than it is likely to be, yet would this afford poor compensation for the loss of the gigantic Patagonians, whom recent voyagers have reduced to little more than the ordinary level of the species. The new animals and plants fetched from remote lands have severally their analogues, already named and registered, in our cabinets and museums. While the huge bones and vestiges of extinct life, which in all parts of the world have perplexed curiosity and startled ignorance, are now submitted to technical description, and brought under the same strict laws of classification as the living forms that surround us. 
The romance of voyage and travel is therefore well-nigh at an end, nor is it likely anything should hereafter occur to revive it. Utility, in all public undertakings of this kind, is now mainly sought after; - what can be gained to physical science, to colonisation, or to commerce and the conveniences of life. The construction of more accurate charts; - the correct fixing of latitudes and longitudes; - the discovery of new harbours and rivers fitted for navigation;-the sounding of seas, from depths which barely float a ship to the profound abysses of ocean where fathom-line of five miles will hardly touch the ground; - the determination of tides and currents; - observations on winds and storms; - tables of magnetic variation, now so important to the exact science of navigation; - these, and other matters more purely scientific, we find appended in one shape or other to all relations of modern voyages, as the documents of highest interest and value. And rightly indeed so esteemed, looking to the actual condition and future prospects of the world; in which certain eminent and favoured races, foremost in civilisation, are rapidly diffusing themselves with ever growing numbers over regions tenanted before by savage or half-civilised tribes, the fractional remnants of an earlier peopling of the glcbe.

The shores of the great southern continent of Australia have recently given ample scope and object to these expeditions of maritime survey. Our national interests are now indeed so deeply concerned in this vast and most singular country, and in the rapid progress our colonies there are making in population, agriculture, mining, commerce, and all that constitutes the germ of great communities, that there could be no excuse for indifference or inertness as to researches thus directed. Australia may well be described as a land of problems and paradoxes in every part of its physical history. In nothing, however, is it more 
strange than in its human relation to our own small island, on the very opposite point of the globe's circumference. The first English settlers, chiefly convicts, landed at Sydney seventy-four years ago. A population of more than a million now dwells in these colonies; intelligent, energetic, and wealthy; possessing the political institutions and freedom of the mother country; and maintaining all the usages of English social life, even to the very pastimes which amuse and give vigour to our youth.* A file of Sydney or Melbourne newspapers of this day may fairly be counted as one of the most curious documents in the history of our race. They tell us not only of a commerce of unbounded activity, but of literary, scientific, and charitable societies; of parliamentary debates; public meetings and dinners; periodicals, club-houses, theatres, concerts, and races;-in short, a sudden and complete translation of the social usages of England to the opposite extremity of the globe.

We have no room, however, to dilate on these various points, or on those strange peculiarities in its native Fauna and Flora, which distinguish Australia from all other regions of the earth, and well justify Cuvier's expression in reference to them:- 'Ils sont venus étonner les naturalistes par des conformations étranges, qui rompent toutes les règles et échappent à tous les systèmes.' Many of these anomalies are becoming obliterated by the ingress of European life in its different forms. Here, as elsewhere, the white man is gradually displacing the coloured races; bringing with him the animals and plants of another hemisphere to minister

* No more striking illustration of this can be given than the fact that, at this moment (February, 1862), cricket matches are going on in Australia, in which the 'All England Eleven,' already famous from their American triumphs, are contending against the native cricketers of our great Southern Colony. It may be added, as a further trait, that the English Eleven are transported from their home ground at Lord's to the antipodes, at the exclusive expense of the colonial population, 
to the wants or luxuries of his new life. It is not, however, colonisation alone which has peopled and given fortune to this new continent. The rush to the Californian gold region was still going on, when the discovery of still more prolific gold-fields on the flanks of the eastern mountain-chain of Australia - a discovery sagaciously anticipated by Sir R. Murchison, but actually made by Count Strzelecki - directed a sudden stream of migration towards these distant lands, which three months of ocean voyage could not check, and which is still going on to enlarge their population and wealth. This topic however, wonderful though it be in every way, is now too familiar to need our dwelling upon it here. Description has almost exhausted itself in the narrative of Australian progress; and there are parts even of our own island less known to us than the country around Sydney and Melbourne, or the gold-fields of Ballarat and Bendigo.

Still what we thus familiarly know cannot be called more than the margin of the Antarctic continent. From its eastern coast discovery has been extended to distances of 700 or 800 miles from the sea; but this in scanty lines and at the cost of much suffering to the adventurers. The larger portion of the coasts is known only by maritime survey, and the vast interior is still in great part a void in our geography. In several respects, either proved or presumed, Australia has much resemblance to Africa in its physical features ;--a circuit of coast, with very few inlets or gulfs ; the highest mountain ranges on its eastern side; an arid saline desert within, touching in parts on the sea, particularly on that long and dreary line of southern coast, which Mr. Eyre with singular powers of endurance successfully explored. The existence of some great central desert (resembling those which Humboldt has so well classed and described in his 'Essay on the Steppes and Deserts of the Globe'), though not proved by actual passage across it, is attested to us in every way short 
of such proof. Without reciting the names of the many eminent travellers who have been engaged during the last thirty years, officially or otherwise, in their arduous efforts to reach the interior, we may state generally that, from whatsoever point of direction ingress has been attempted, a waterless and barren desert has been eventually reached, frustrating all further advance. In this desert, entering it from the east, we have cause to presume that Leichardt and his companions perished. Of its boundary on this side we now know more from the recent and remarkable journey of $\mathrm{Mr}$. Gregory; who, going in search of Leichardt, traversed the continent by the long inland line from Moreton Bay (now the new colony of Queen's Land) to Adelaide, in South Australia. This traveller had already gained reputation and the gold medal of the Geographical Society for his eminent services in the North Australian expedition of 1855, which, entering the continent at the mouth of the Victoria River, proceeded to the S. and SW., until arrested, about 300 miles from the coast, by the same wilderness which had already so often baffled the enterprise of preceding travellers.

Other physical proofs of the generally desert character of this central region are afforded by the paucity and scanty size of the Australian rivers, of which the Murray alone seems fitted for continuous steam navigation; and by those dry, scorching and sandy winds, blowing from the interior, which are so forcibly described by all recent colonists. The suspicion of a great central lake, though it cannot actually be disproved upon our present knowledge, yet is now entertained by few. Many wide tracts of fertile country, profitable for settlement, will doubtless yet be found between the desert and the sea; of which the recent discoveries of $\mathrm{Mr}$. Stuart to the north-west of Lake Torrens furrish a fortunate

- example. But taking its physical characters in their totality, Australia seems destined to become a Coast Empire only; 
though vast enough, as such, to satisfy all the imaginings of the future.*

It is impossible, indeed, not to feel pride in the relation of England to this southern world. Whether it remain ours in colonial union, or be peaceably dissevered by the events of the future (peaceably we say, because such a war as that of the American revolution can never recur), Australia will still be English in its language, literature, and political life. We shall not only have created a nation out of a wilderness, but shall have ennobled the people so created, by the gift of institutions which the struggles and experience of centuries have happily won for ourselves. It is a gift not without its perils to an infant community; but which, well and wisely watched over in this early time, offers the best pledge for future greatness.

Though the sovereignty of Australia is virtually conceded to England, and the most profitable parts of its territory already occupied by our colonies, we are led with Sir R. Murchison to regret that no settlement has been made on the northern coast. The proximity to the equator is a drawback; and we have reason to suppose the margin of fertile lands narrower and less productive. Still we mist believe that on the shores of the Gulf of Carpentaria, or elsewhere on this coast, there are spots profitable for colonisation, for commercial intercourse with the Indian Archipelago, and for

* 1862. Another great step has recently been made in Australian discovery by the accomplished passage across that continent; - if not actually from sea to sea, yet within sight of the tidal waters of the northern coast. The successive expeditions of Mr. Stuart and Mr. Burke (the former successful to its close, the latter painfully disastrous in its end) have indicated a line of route in which, though desert tracts have to be crossed, water and vegetation are never long wanting to the traveller. Inspection of the map of Australia, however, will show how vast a void is still left in its geography, to the west of this central line of transit; and speculation, though somewhat narrowed in its scope, may yet be kept up as to the interior character of a region scarcely less than European Russia in superficial extent. 
future overland communication with the northern settlements of the eastern coast. The energy, which has done so much for us in this region of the globe, will doubtless in the end accomplish the object to which we allude.

The voyage of Captain Blackwood, narrated in the volumes before us, was undertaken by direction of the Board of Admiralty, and extended over a period of more than four years; - great part of this time occupied in a laborious, and oftentimes dangerous survey of one of the most singular channels of navigation in the world. The quarter to which his operations were directed is the north-east coast of New Holland, of which the line of travel previously pursued by Leichardt may be said to form the interior chord. These two expeditions therefore have been in some sort supplemental to each other, and to the survey of the Gulf of Carpentaria by Captain Stokes in the years immediately preceding. But the definite object assigned to Captain Blackwood was the complete survey of the channel or channels just mentioned, through which a hardy and prosperous traffic is already beginning to flow, and which are likely hereafter to become one of the great passages for the commerce of the Indian Archipelago and Southern hemisphere. We infer from these volumes, as well as from other information which has reached us, that this officer fulfilled well the arduous duties intrusted to him; proving himself a worthy successor of Flinders, Bligh, King, Stokes, and other navigators who have laboured in the work of discovery on the same shores.

It will be seen from the title of the volumes that Captain Blackwood is not the historian of his own voyages. He waived his right of publication in favour of Mr. Jukes, naturalist to the expedition, who in a modest prefatory letter acknowledges this kindness and apologises for the deficiencies of his work. In an Appendix to the second volume we find 
a copy of the Admiralty orders under which Captain Blackwood sailed; a perspicuous document, and excellent not only in the explicit nature of the naval instructions, but also in its humane and judicious inculcation of rules for intercourse with the natives. The following are stated in this document as the main objects of the expedition :-

The survey of the exterior or eastern edge of that vast chain of reefs, which extend almost continuously from Breaksea Spit to the shore of New Guinea :

The thorough examination of all the channels through the barrier chain, with detailed plans of those which offer a secure passage, and the device of some practical means of marking them by beacons of wood, stone, or iron:

And the ascertainment of the safest channels by which vessels coming from the eastward may pass through the intricate reefs and islands occupying the mouth of Torres Strait; and, in particular, a complete survey of the passage cailed Endeavour Strait.

These orders are closed by the following passage, which well deserves every attention:-

But, wherever you go, we expect you to produce full and faithful surveys of the places you visit. And we especially desire you not to waste your time and means in what are called running surveys, in which much work is apparently executed, but no accurate knowledge obtained, useful either to the mariner or geographer. Whatever you do is to be done effectually.

This is sound and vigorous instruction; and applicable not solely to voyages of discovery, but much more largely to all great enterprises and objects of human pursuit.

We must carry our readers somewhat farther into the description of this Great Barrier Reef, not merely as forming the main object of Captain Blackwood's expedition, but from its being marked as the most singular and gigantic example of its kind on the surface of the globe. Among the various 
phenomena of physical geography, few in truth are more extraordinary than those great coral formations, which, under different shapes and designations, meet the navigator in his passage through the tropical seas; - rarely passing far beyond these limits of latitude, but, within the wide belt of ocean thus included, rising up from unknown depths in stranger forms than imagination could devise, and alike perplexing to the naturalist from their multitudinous occurrence in some tracts of sea, and their absence in others. Here we find the circular lagoon islets (or atolls, as they are now termed, by adoption of a native word), circles of coral rock, often barely emerging from the wilderness of waters around; yet resisting the heaviest storms, and sheltering small central lakes, the placid surface of whose blue water strangely contrasts with the tumult of waves without. Elsewhere, as in various parts of the Indian, Pacific, and Atlantic oceans, we see these coral islands occurring in closer groups, with innumerable channels between; covering often a wide area of sea, and so numerous as almost to defy all reckoning and survey. England, which plants its flag on every various surface of the earth, possesses in the Bermudas one of these coral clusters, further remarkable as the most distant point from the Equator at which coral rocks are known to occur. Elsewhere again, we find these extraordinary creations of the deep forming barrier reefs to islands or portions of continent; encircling some, bordering or fringing others, through lines of enormous extent. In certain places, as between the north-eastern coast of Australia and New Caledonia, they are so largely developed in the form of detached reefs as to have obtained from Flinders the name of the Coral Sea.

To almost all our readers it must be known that these vast works are due to the labours of certain species of Zoophytes; ranking anong the most minute and slightly organised forms of animal life, yet having a common instinct of existence 
which renders them the artificers of mineral masses and new lands amidst the ocean, fitted eventually to become the abode of man. The soft pulp of the coral animal secretes, or otherwise elaborates, a stony nucleus; the aggregation of which matter, by the conjoint working of myriads of these little creatures, and the accumulated and superimposed labours of different species and successive generations, produces these wonderful results : - 'admiranda levium spectacula rerum,' as they may well be termed, looking at the relation between the agent and the magnitude of the work accomplished.

In a later part of this article we shall have to refer again to this topic, as connected with the theory of coral formations and their relation to other great physical phenomena of the globe. Meanwhile we will merely remark, that the whole course of modern science tends to disclose facts analogous to those just mentioned. We everywhere see the wonderful agency of the organic life of former ages, in forming the material and determining the structure of the great masses which compose the crust of the earth; as well as in producing other phenomena, apparently the most alien from such origin. Where formerly brute matter alone was seen or suspected, the eye of the microscope now shows the innumerable relics of living beings, the artificers of the very mass which thus entombs them. The flint nodules of chalk rocks, the hard Tripoli slate, even certain varieties of the noble opal, are composed, in part or wholly, of the siliceous cases of fossil infusoria. The sand which sometimes falls on ships far distant from the coast - the mud which lies in the estuaries of rivers - even the layers of ashes and pumice which cover the edifices of Pompeii - give the same remarkable result. We look backwards through these ages of organic life on the surface of the earth; and in the very minuteness of forms and species we find reason why they should have been thus readily aggregated into dense masses, concealing to common 
observation the vitality which once pervaded the whole. Looking forwards, we see the earth and seas still teeming with the same profusion of life in its simpler forms, and cannot but infer that these may hereafter undergo the same changes and minister to the same great results.* Science stands here, as in so many other instances, between the past and future time; casting upon the latter the light, more or less distinct, which it derives from reflection of the former. It may be that the period of all greater terrestrial revolutions has come to a close; and that man is destined henceforward to live in comparative tranquillity on the globe. But in the phenomena of earthquakes and volcanoes - in the gradual upheaving or subsidence of lands - and in the heat of deep mines and Artesian wells, we have evidences of subterranean actions still going on which cannot be wholly inoperative on the surface. The bed of the Ocean, moreover - that great recipient of all deposits organic and inorganic, the débris of the earth - may be exposed to physical actions from underneath, of which we have no certain knowledge, but which time, calculated by ages, may render of great account in the futurity of our globe.

Recurring to the subject more immediately before us, we would beg the reader to take up the map of New Holland, and to fix his eye on Sandy Cape, in S. lat. $24^{\circ} 30^{\prime}$, about 600 miles north of Sydney, and the most salient point on the

* We may mention, as it is not generally known, that Ehrenberg has actually succeeded in producing Tripoli and polishing slate from living infusoria. We may further add that he found in a peaty argillaceous deposit, twenty feet kelow the pavement of Berlin, masses of infusoria still living, and in some places deposits of ova reaching to much greater depth. In the public gardens at Berlin workmen were occupied many days in removing masses wholly composed of fossil infusoria. In the moors of Leinburg there occur similar accumulations twenty-eight feet in thickness. Observation probably is alone wanting to multiply indefinitely facts of similar kind; but the inferences which these, and other wonders of the fossil world, have already furnished, may well be counted among the triumphs of modern science. 
eastern coast of the Australian continent. Some miles northwards from this Cape begins the Great Barrier Reef; the gigantic dimensions of which will be understood by carrying the eye along the Australian coast to Torres Strait and the shores of New Guinea, and learning that this coral reef forms a continuous barrier, separating an inner and shallow coast channel from the deep sea without, and stretching throughout the whole length of the line just described. A mere inspection of degrees of latitude will show that this length exceeds 1,200 miles; and the term continuous is justified by the fact that, except towards the southern extremity of the line, it is broken only by narrow channels or gaps. Still, in strictness, the chain must be considered as a series of individual coral banks of greater or less extent, assuming this definite rectilinear direction parallel to the line of coast. The channel between the barrier thus formed and the mainland contains some scattered reefs; the outer, or ocean side, dips down precipitously to depths yet unfathomed, leaving a clear sea to the east from 60 to 100 miles in width. Beyond this, in the direction of New Caledonia, coral islands or reefs again appear, in unknown number and variety of form, scattered over what we have already noticed under the name of the Coral Sea.

Our author, in the chapter of his volume before alluded to, well describes the general aspect and character of this great boundary-reef or submarine buttress; the width of which, near the surface, appears to vary from 280 yards to more than a mile.

If it were to be laid dry, this great barrier would be found to have a considerable resemblance to a gigantic and irregular fortification - a steep glacis crowned with a broken parapet-wall, and carried from one rising ground to another. The tower-like bastions of projecting and detached reefs would increase this resemblance. From examination of our charts it would appear that the normal condition of this long mass of reefs is that the outer barrier should 
be narrow, rising precipitously from a great depth, and running more or less nearly in a straight line; and that inside this outer barrier there should be a clear space about twenty fathoms deep and several miles wide, between which space and the land should be another body of reefs. - Vol. i. p. 332.

Thus there may be said to be two channels connected with the Great Barrier Reef; - the Inner one having an average width of about thirty miles, though narrowed by subordinate reefs on the land side; the navigable part of it varying in depth from ten to thirty fathoms, safe in being thoroughly sheltered from the storm of the ocean without, and affording good anchorage wherever the channel is narrow or intricate. The Outer passage, on the other hand, though giving a wider route for navigation, has a profound depth of sea without the possibility of anchorage; so that under no circumstances in which a vessel is placed can refuge be had, unless she is able to make her way through one of the openings in the Great Barrier, and thus to find access to the sheltered sea within. In this general description will be seen the objects and value of the survey now completed; which, by ascertaining the openings of easiest access, and of width and depth best suited to navigation, and by fixing beacons to mark and distinguish them, gives great increase of safety and facility to ships traversing these seas. The probable number of these openings or transverse channels we do not find anywhere denoted. In truth, it would be difficult to enumerate them, seeing that they vary from mere fissures in the reef to passages several miles in width. The good ship-channels alone have value, and these seem to be comparatively rare.

But the objects of this survey would not have been fully attained, without a thorough examination also of that northern portion of the Great Barrier reef which stretches well-nigh across Torres Strait; and intercepts, though in a 
more complex and irregular manner, the free passage from the Southern Pacific to the seas of the Indian Archipelago. A moment's inspection of the map will show the singular importance of this Strait to the direct intercourse between our great Australian colonies and India, China, and Europe; and the value of an accurate survey of its complicated and difficult channels, so strongly enjoined in the Admiralty instructions for Captain Blackwood. It is, indeed, a strange and uncouth passage - a labyrinth of coral reefs, volcanic rocks, islets, and shoals - yet destined nevertheless to yield that free channel which man requires for his commerce, and which the zeal and boldness of those seeking for it are sure eventually to obtain.

Having dwelt thus fully on the local circumstances of reef, channels, and sea, which formed the object and guided the direction of Captain Blackwood's survey, we need not pursue the track of his voyages in any minute detail. The actual survey was begun at the end of the year 1842, eight months after his departure from England, and his labours were continued until June, 1845; - with intervening periods of repair and repose at different parts of Australia and the Indian Archipelago. These labours, combined with those of Flinders at a prior time, complete the survey of the Great Barrier Reef for a space of nearly a thousand miles, leaving only one short hiatus to be filled up. The examination of the eastern part of Torres Strait, and the channels amidst its reefs, is perhaps the most valuable part of the work accomplished; and the charts reduced from the survey will remain as lasting records of it, unless some of those gradual changes on the crust of the globe which geology records or other more violent and sudden convulsions, such as have been frequent among the Indian islands - should disturb the coral flooring of these seas, and alter the soundings and channels that have now been explored. 
In a part of this great barrier, between lat. $11^{\circ} 20^{\prime}$ and $12^{\circ} 20^{\prime}$, the line of reefs, instead of being straight or gently curving, is sharply deflected into convolutions, forming deep bays with detached reefs outside. In one of these bights, having an entrance six miles wide and stretching eight miles inwards, such is the depth that no bottom was reached except close to the reefs, though a line of nearly 300 fathoms was thrown out. This extraordinary spot was called Wreck Bay; and with melancholy fitness of name, as Captain Blackwood found lying on the reef near it the wrecks of two large vessels (the Ferguson and Martha Ridgway), lost here in 1840 and 1841 ; the former having part of the 50th regiment on board. Happily the presence of another vessel in company prevented in this case any loss of life. In singular contrast to this disastrous history of this place, our author gives us the following vivid description of one of the coral reefs in its close vicinity :-

In a small bight of the inner edge of this reef was a sheltered nook, where the extreme slope was, well exposed, and where every coral was in full life and luxuriance. Smooth round masses of mæandrina and astræa were contrasted with delicate leaf-like and cup-shaped expansions of explanaria, and with an infinite variety of branching madreporæ and seriatoporæ; some with mere fingershaped projections, others with large branching stems, and others again exhibiting an elegant assemblage of interlacing twigs, of the most delicate and exquisite workmanship. Their colours were unrivalled - vivid greens contrasted with more sober browns and yellows, mingled with rich shades of purple, from pale pink to deep blue; bright red, yellow, and peach-coloured nulliporæ clothed the masses that were dead, mingled with pearly flakes of eschara and retepora, the latter looking like lace-work in ivory. In among the branches of the corals, like birds among trees, floated beautiful fish, radiant with metallic greens or crimsons, or fantastically banded with black and jellow stripes.

Fortunately for a spot which has acquired a melancholy notoriety by these and other wrecks, the discovery was made 
within a very short distance of an excellent channel traversing this outer line of reefs; and the erection of a lofty beacon on Raine's Islet, at the edge of this passage, may be cited as one of the main objects fulfilled by the expedition. The work was begun in June, 1844, and completed in less than four months. Twenty convict masons and quarrymen were brought from Sydney; a quarry was opened in the coral rock; lime was got by burning sea-shells; wood for burning was brought from islands near the mainland; water procured from other islands, 25 miles distant; and timber for the building obtained from the wreck of the Martha Ridgway. Having no anchorage nearer, the 'Fly' had to lie 12 miles off, within the reefs of the barrier, the smaller vessels and boats running to and fro with the various provisions and materials needful for the workmen. Under all these difficulties a strong circular stone tower was erected, 40 feet high and 30 feet in diameter at its base, raised 30 feet higher by a framework of wood covered at the top with painted canvas. Mr. Jukes describes the little islet which gave foundation to the work - a spot not two miles in circumference and scarcely 20 feet above high-water mark. Seeing the strangeness of the situation and the peculiarity of the work, we cannot but believe that it might have furnished some amusing incidents to a narrative which is very deficient in these points. The truth seems to be that the time was one of dull inaction to those not engaged in the erection, and that they looked upon it with weariness and distaste.

Accordingly we find our author, when the beacon was half completed, starting in a vessel for Cape York, - the great promontory which abuts on Torres Strait, forming the N.E. point of the Australian continent. We have already mentioned that surveys were effected of this Strait and the channels traversing its isles and reefs, the complexity of which renders it, as we have seen, one of the most dangerous, as it 
is one of the most important passages on the globe. The examination of Endeavour Strait, that channel which winds immediately round Cape York, was made with all the exactness enjoined by the Admiralty, and justified by its peculiar importance. The line of steam navigation long and loudly invoked for the Australian seas, in connection with those great lines which already unite India, China, and Europe, has hitherto been directed to the southern coasts - a natural effect of the rapid growth and high commercial activity of the settlements in the south-eastern part of Australia. The channel of Endeavour Strait will doubtless, however, be resorted to hereafter in connection with the ever-growing traffic of the Indian Ocean, and the rising demands of our colonies continually spreading northwards in this direction. Each separate sounding, or observation of wind, tide, or current, now made, must bear upon the security and welfare of this future navigation.

These circumstances and a regard to the remarkable position of Cape York - commanding, it may fairly be said, this great highway between the Pacific and Indian Oceans - lead our author to discuss a question, which has for some time been before both home and colonial authorities, viz. the relative value of Cape York and Port Essington, as a post and settlement for aid to the navigation of these seas. The latter place (600 miles to the west of Cape York, and on the other side of the great Gulf of Carpentaria) was for several years occupied by an officer and a few marines; but without any attempt at colonisation, and with little encouragement to it, either from fertility of the soil, healthiness of the spot, or the character of the natives in its vicinity. Repeated visits to both places have given Mr. Jukes a very decided preference for Cape York; and in his reasons for this preference we fully concur. Its position is a main point in its favour. It is in the vicinity of danger, and affords a 
place of easy refuge. In war it would command security to a passage where a single enemy's ship might inflict much mischief. Its distance from Sydney, 1,700 miles, would make it an admirable depôt for coal in the steam navigation towards India. Though without a harbour this is little needed, since the reefs. themselves, which create the danger of the Strait, protect the sea from all heavy swells and render anchorage secure. At Evans's Bay, which is suggested as a site for the settlement, there are dry rocky places for building, pools of fresh water, and a considerable surface of fertile land; frequent rains, fresh breezes, and a healthy vegetation. All these advantages are absent at Port Essington; and putting the matter on a personal footing, Mr. Jukes declares that if condemned to either; he would rather live at Cape York for five years than at Port Essington for two; an odd numerical formula of preference, but sufficiently intelligible to us.

The right manner of solving the question we believe to be, that Cape York should be adopted as a new settlement, and Port Essington not abandoned. Mistress of Gibraltar, Aden, and Singapore, and fully cognisant of the value of such positions for commerce and power, England can hardly choose but plant her flag on a promontory which commands the passage between two oceans. But the whole shores of the Continent of which it is one extremity, are becoming hers by discovery and colonisation; and in relation to the future commerce of the Indian Archipelago, some more western points on this northern coast require to be occupied, even though offering little present benefit or promise. The active spirit which at this time pervades the world, working with new means and appliances of every kind, will vivify in the end what is barren now ; and no expenditure or labour can be better bestowed than in aiding by anticipation the progress which commerce and colonisation are making on these distant shores. 
While upon this subject, we must indulge ourselves with a short parenthesis as to that extraordinary line of steam communication between England and her Eastern possessions (somewhat strangely called the Overland Journey), of which Australia and New Zealand will in future form the extreme branches. The creation of the last twenty years, this communication has already acquired a maturity of speed and exactness, notwithstanding the enormous distances traversed, and the changes necessary in transit from sea to sea. The Anglo-Indian mail, in its two sections, and including passengers and correspondence, possesses a sort of individuality as the greatest and most singular line of intercourse on the globe. Two of the first nations of Europe, France and Austria, struggled for a time for the privilege of carrying this mail across their territories. Traversing the length of the Mediterranean, it is received on the waters of the ancient Nile;-Cairo and the Pyramids are passed in its anward course; - the Desert is traversed with a speed which mocks the old cavalcades of eamels and loitering Arabs; it is re-embarked on the Red Sea near a spot sacred in Scriptural history; - the promontory projecting from the heights of Mount Sinai, the shores of Mecca and Medina are passed in its rapid course down this great gulf; - it emerges through the Straits of Babelmandel into the Indian seas, to be distributed thence by different lines to all the great centres of Indian government and commerce, as well as to our more remote dependencies in the Straits of Malacca and the Chinese seas. There is a certain majesty in the simple outline of a route like this, traversing the most ancient seats of empire, and what we are taught to regard as among the earliest abodes of man, while now ministering to the connection of England with that great sovereignty she has conquered, or created, in the East; - an empire more wonderful, with one exception, than any of those of antiquity; 
and possibly not less important to the future destinies of mankind.

During Captain Blackwood's survey of the Great Reef Channel and the rivers entering it, there was frequent communication with native tribes, which Mr. Jukes relates in some detail. Though certain peculiarities of usage are noticed, we find nothing in these relations which differs materially from the description familiar to us in the narratives of former travellers, regarding their intercourse with this people. They seem, as here described, to be a tall and athletic race, active and bold in their demeanour and habits; with an occasional fierceness of temper, of which a melancholy proof occurred in the death of one of the seamen of the expedition, struck by a native spear. In describing the feelings excited by this event among the others of the party (making them reluctant to leave the coast without some opportunity of revenging their comrade's death) our author explains the source of many of those unhappy atrocities which still occasionally deface our intercourse with the native Australians, despite the higher and better views now governing our colonial system. On the outskirts of the settlements such occurrences have always been more frequent, from obvious causes in the character and habits both of the white and native border population. And we cannot but fear that the spread of colonisation in the N.E. portion of Australia certain eventually to occur - may involve a repetition of such calamities, seeing the masculine character of many of the native tribes on this part of the coast. Time will in the end put a stop to all these things; but it can happen only through that extinction of the native population, which, by a strange and sad destiny - the ineluctabile fatum of what we call savage life - seems always to occur sooner or later where Europeans have trodden upon new lands thus primitively peopled. 
In the latter part of Mr. Jukes's first volume we, find narrated the proceedings of the expedition on the southern coast of New Guinea; which coast, with its widely spread banks of shoal-soundings, was surveyed for a length of 140 miles. It is a small section indeed of the shores of this vast island, but important as the northern boundary of Torres Strait; and further interesting as some slight index to a country less known perhaps than any other of equal size in the habitable world. Notwithstanding its great extent, equal to that of Great Britain and France conjointly - its remarkable position in the Eastern Archipelago - its proximity to islands long visited or colonised, and to channels of great and increasing commerce - the outline of Papua, or New Guinea, still remains to be completed in our maps, and we have no certain assurance whether it be a single island or several. Of its interior we are yet almost wholly ignorant; discovery having never advanced more than a few miles from the coast, and this more as a passing adventure than on any deliberate plan of survey. Bát enough has been seen, or learnt through indirect channels, to indicate a region of luxuriant tropical vegetation; - profuse in its various forms of animal life; - abounding in water, large rivers, and mountain-chains; - a striking contrast, in short, in all physical conditions to the adjacent continent of Australia. Such features as these, occurring close upon the Equator, and in a country 1,200 miles in length, give promise of an exuberant harvest to the naturalists who may hereafter find access to the Fauna and Flora of this unknown land. No long time can now elapse before this access is found.

The channel of Torres Strait, remarkable in so many ways, is not least so in the sudden line of demarcation it thus draws between two kinds of vegetation, two groups of lower animals, and two varieties of the human race. Those strange anomalies which designate all that belongs to Australian landscape 
and life, extend even to the isles bordering this continent within the Strait. But in the isles and land of New Guinea, scarcely 50 miles distant, the wonderful change we have just denoted comes suddenly over the scene, both as respects vegetable and animal life; extending even to the shells and echinodermata which lie upon the shores.

The geology also of the coast and islands of Torres Strait possesses much interest. These islands are evidently points in the submerged prolongation of that great mountain-chain which, rising from the Southern Ocean in Van Diemen's Land, sinks again under the sea in Bass's Strait, with the exception of a few island points left above the waters; - then emerges in lofty masses at the S.E. extremity of New Holland, forming the mountain group which Count Strzelecki was the first to survey; - stretches along the whole eastern side of this continent, a range of more than 1,600 miles; at Cape York again sinks underneath the sea of Torres Strait, reappearing at Mount Cornwallis on the New Guinea coast, beyond which it is lost to our present knowledge,* The axis of this great chain is composed of primitive rocks, chiefly unstratified, flanked by Palæozoic strata incumbent on them, and mixed with rocks of eruptive character; - and succeeding to these in order of time, certain detached coal formations, and superficial beds representing the tertiary formations of Europe. We infer from observations in the work before us, that the same general character extends to

* There would be wrong done in naming Count Strzelecki, without adverting to the work on Australia by this very eminent traveller and naturalist. The five years he passed in the country, during which time he travelled over 7,000 miles, almost entirely on foot, were sedulously and successfully employed in studying in all its parts the natural history of this singular region. The results, many of them of high value, are embodied in the volume he published in 1845. They include all that belongs to Physical Geography and Natural History in the largest sense of the terms; and much that is important also in regard to the statistics of the Colonies at that time, and the condition of the Aborigines of the country. 
the northern extremity of the chain. Cape York and the adjacent isles are porphyritic, and the islands which traverse the strait in the same line appear to be all composed of granite, sienite, or old metamorphic rocks. A circumstance well worthy of remark is, that to the eastward of this line none of these primitive rocks appear, but low coral isles or coral reefs occupy solely a belt of sea, sixty miles wide, across the mouth of the Strait. To the east of this again all the islands are volcanic, and chiefly composed of lavas. The distinct division by these three belts adds another to the many singularities of this great ocean channel.

In the foregoing part of this article we have drawn somewhat largely upon our readers' attention (perchance also a little on their patience) by the various facts connected with the coral ridges and reefs forming the vast and prolonged line of barrier on the Australian coast. We recur for a short time to the subject; not, however, in relation to particular localities, but to the general history and theory of these coral formations as one of the great physical phenomena of the earth's surface:- impressive, moreover, not solely from the enormous magnitude of these animal creations of the Ocean, but also from the index and evidence they afford of past and progressive changes in the level of the solid crust of the globe. In a former article of this Review (on the Voyages of the Adventure and the Beagle), we noticed the striking views as to these coral formations contained in Mr. Darwin's journal of the latter voyage. This eminent naturalist has since published a separate volume, 'On the Structure and Distribution of Coral Reefs,' which we have taken as one of the heads of the present article; wishing to complete the view of the subject, and seeing that to Mr. Darwin we owe not only the most extensive and exact observations upon it, but also certain general conclusions which are now in progress of adoption by men of science in every country. From 
this volume, which possesses the charm of a simple and perspicuous style, conjoined with great reasoning powers, we shall briefly extract some of these conclusions, as well as the more important facts from which they are derived.

We have already noticed generally the three classes of atoll, barrier, and fringing reefs, including all the most characteristic varieties of coral formation on the globe. These varieties, however, owing to local or other conditions, are so multiplied in detail, that it would be almost as difficult to give a clear description of them, as to explain the circumstances in which they respectively originate. Without attempting to follow Mr. Darwin in his more ample survey, we may state that he selects as instances of the lagoon islands or atolls, Keeling Island in the Indian Ocean, the vast group of the Maldives, and the extraordinary submerged atoll called the Great Chagos Bank. The first is a single but characteristic specimen of its class. The Maldive Islands form an archipelago of coral atolls, 470 miles in length and about 50 miles in breadth; the atolls ranged in a double line, and some of them of great size - as that of Suadiva, 44 miles by 34 , with an included expanse of water nearly 300 feet deep, and not fewer than 42 channels through which a ship may enter this central lagoon. The Chagos Bank, in the centre of the Indian Ocean, rising abruptly from unfathomable depths to a level near the surface (its longest axis of 90 miles, its breadth from 50 to 70), is well described by Captain Moresby as 'a half-drowned atoll;' a view confirmed both by soundings, and by the many similar reefs and atolls rising to the surface around it. To this officer we owe admirable surveys of the Maldive and Chagos groups, which have done much to illustrate the subject.

Of the 'Barrier-reef' the most conspicuous instances selected are that great reef, fronting the eastern coast of Australia, with which our readers are now acquainted; and a. 
similar but shorter one on the western coast of New Caledonia. The term, however, has been fitly extended by Mr. Darwin to those reefs encircling smaller islands, which are so numerous in the Pacific, and familiar to us in the narratives of voyages in this ocean; - coral walls in fact, with a deep moat within, circling round islands of every various dimension and height; - some little raised above the sea - others like Tahiti, having an elevation of many thousand feet.

'Fringing or Shore reefs,' whether encircling islands or portions of continents, differ from those just described in being less massive, in having no interior deep-water channel, and in sloping downwards into the sea upon the natural declivity of the shores. The reefs of the Mauritius furnish a well-marked insular example of them. The coasts of Brazil and Arabia afford instances, among many that might be quoted, of such coral fringes to continental lands.

Mr. Darwin has done much to simplify the view of the several coral formations just noted, by showing that they graduate into one another; and that the atolls, barriers, and encircling reefs are but modifications, deviating much in the extreme cases, of a common principle and manner of operation. A perfect series, in fact, can be traced from the simple linear or normal state of the reef, to the long linear lagoon, and thence to the oval or circular form of the encircling reef or the atoll. Again, if from the barrier reefs encircling small islands we abstract the land within (a legitimate speculation, as will hereafter be seen), we bring them to an almost complete identity with the simple atoll or lagoon island, in form, dimensions, and grouping. The value of such generalisations as these to a just theory on the subject will be well understood, even without a full comprehension of the details on which they are founded.

The fourth chapter of Mr. Darwin's volume relates to the distribution of coral formations, and their rate and manner 
of growth : including such knowledge as we possess regarding the species and habits of the polypifers, or coral animals, which have produced them. On the subject of these wonderful zoophytes, the details given are not perhaps as distinct as might have been desired for general information. It is Mr. Darwin's main object, in reference to his theory, to determine the rate as to time, and the depth below the surface of the sea, at which the workings of living coral can go on; and we do not find in his volume any consecutive description of the aspect, species, and habitudes of these active tenants of the deep. It must be admitted, indeed, that our knowledge on these points is very deficient. Exact observations are not easy where the animal works either below the surface of the water, or amidst the heaviest surf and breakers on the edge of the reef. Here, where all besides perishes, the zone of coralline life exists in its greatest activity; but hardly more accessible to observation than are the dead corals brought up by dredging; - in such variety of species as to leave it uncertain which are the true artificers of the reef, and which contribute to it solely by being agglutinated, with broken coral, sand, shells and other casual materials, into the common mass. Ehrenberg has described more than a hundred species of coral which he found in the reefs of the Red Sea. Some observers have believed that the genus Astræa is that most' efficient in the formation of coral rocks. But the Madrepore, Millepore, Porites, Meandrina, Caryophyllia, and various other genera contribute largely to these great and mysterious works; - in what proportion, and under what laws or instincts of combination, we shall probably never be able fully to comprehend.

Other problems equally difficult embarrass this subject; and notably in the first place the question, why coral reefs should be so vast and numerous in certain tracts of ocean, while others are wholly destitute of them? The limitation 
to tropical latitudes is intelligible; but why, with the exception of the Bermudas, there should not be a single coral isle or reef in the great expanse of the Atlantic, it is hard to explain. Had the Bermudas themselves been absent, a certain general conjecture might have been hazarded, which their actual position and coralline structure render inadmissible. Volcanic action, ancient or recent, affords no explanation of this partial distribution; nor do any ascertained differences in the depth or bottom of the sea, or in the mineral contents of different seas, come in aid of the solution. A.supposition is still open, vague perhaps in its application to the present case, yet supported by many analogies drawn from other parts of the animal world. The corals, in their pulpy portion, afford food to several species of fish and to the whole tribe of Holothuriæ; while they themselves, simple and minute though they be, must feed on some other kinds of organic life. There may be in certain seas a predominance of the animals destroying them, or a deficiency in those affording them nutriment. In either of these conceivable cases we bring the question among those curious instances (now almost forming an especial branch of natural history) in which the existence, range, multiplication, or extinction of species, are determined, not merely by inanimate agents around, but by the presence or absence, abundance or scarcity, of other species in the same regions and at the same periods of time. We might say much as to this interesting and prolific course of enquiry, did it come within our present subject.

The manner and rate of growth of corals offer other curious questions to the naturalist. The evidence as to the latter point is various, and on first view somewhat contradictory. With some well-attested examples of rapid growth (the filling up of channels and lagoons, and enlargement of islands within human record) we have other instances where 
the same surface and elevation of living coral appear to have existed for ages unchanged. Ehrenberg found in the Red Sea vast globular masses of Meandrina, which, he says, are of such antiquity that 'Pharaoh himself may have beheld them;' and he gives evidence to show that various coral formations of this sea have undergone little or no change within the last two centuries. Captain Beechey furnishes similar proofs from different parts of the Pacific. Resting upon these and other instances, some naturalists have been led to view the growth of corals as the slow work of ages rather than of years, and to doubt the possibility of islands having been thus formed in the midst of the ocean.

Such difficulties may unquestionably be lessened, if not removed, by a regard to the various conditions under which coral masses are formed; - by the difference of the coral animals themselves in species, size, and habitudes of existence; - by the succession of several species in the same mass; - by the important fact (ascertained as far as negative proofs will carry us) that new coral does not form on the surface of that which is still living; - by the various foundations on which the corals build their superstructure; - and by the changes of level, sudden or slow, occurring in these foundations. We incline, therefore, to Mr. Darwin's belief that the formation of coral is still actively proceeding in numerous places over the globe. We find no reason to doubt that the atolls and reefs rising precipitously from the deep ocean around, whatever of increment they may receive to their growth from other sources, are mainly, as we see them, the creation of successive generations and species of these zoophytes. In the whole range of physical causes we find, in truth, none but this strange and instinctive workmanship - this antagonism and superiority of organic vital forces to the inanimate powers of nature - which can explain such phenomena as those of the Maldive group; or the simple 
fact of the small circular coral islet rising up to the surface of the Pacific from unfathomed depths around it; or other singularities of these constructions which we have no room to detail, but which are fully described by naturalists.

These considerations bring us nearer to the actual theory of coral rocks, but with the intervention still of another question (of great moment, as we shall see, to any general conclusions), viz. the depth of ocean at which the reefbuilding corals can live and work? This, it will be obvious, is a question distinct from that of the gross thickness or depth of coralline masses, and somewhat easier of solution, yet not without its appropriate difficulties. From a large collation of facts Mr. Darwin is led to conclude that, in ordinary cases, the corals which build reefs do not flourish at greater depths than from 20 to 30 fathoms, and that the greatest activity of their existence is on the surface and outer edges of reefs. Other observers have limited their range of operation still more closely to the surface; but take what estimate we may, it seerns certain that no increment can take place to coral growth below a comparatively small depth; - none whatsoever above the surface washed by the spray of the sea. Whence, then, the rast masses and lofty coral pinnacles which the sounding-line follows downwards to the depth of several hundred fathoms, with evidences of the same structure and origin, and leaving it uncertain whether they may not descend deeper still? If these zoophytes work only thus near to the surface, how are we to explain the origin and actual position of all that lies beneath? This is the problem most interesting in the theory of coral formations; and the solution of which, whatever it be, associates them most closely with the great geological phenomena of the globe. In the article before alluded to we have given an outline of the question and of Mr. Darwin's views upon it. In the short space now remaining to us we shall put 
before our readers a summary of the discussion as it at present stands, with such few remarks as we think more especially conclusive on the argument.

The hypotheses by which alone we can seek to solve the problem just stated are few in number. Either the.corals constructing the lower parts of the reefs must be wholly distinct in species and habits from those which work near the surface; - or the reefs, atolls, and islets we see must be mere superficial coverings or cappings of points and ridges of land underneath; - or, thirdly, there must have occurred such subsidence downwards of the land encircled by or supporting coral formations, as to leave the coral summits solely on the surface of the waters; with means of increment, where the subsidence further continues, by the superinduction of fresh layers, under the conditions of depth favourable to the living actions producing them. We are unable to find any other suppositions than these which will apply to the solution of the problem before us.

The first of them is negatived in great part by the improbability that there should be species of corals differing so widely as to one of the most important conditions and necessities of their existence; and further, by the negative fact that no examination of the dead coral taken up from great depths has disclosed such varieties.

The second hypothesis is of more plausible kind, and was at one time adopted by Sir C. Lyell, in common with many other naturalists; but subsequently relinquished by this eminent observer in favour of the last of the opinions just stated. It was, in truth, a natural and easy conception that the coral formations incrusting the upper surface might follow and depicture the outline of the submarine bottom, and the peaks and ridges rising from it. And this argument became more specious when considering the coral islets, with their circular and often deep lagoons within, as representing the cones and 
craters of old submarine volcanoes; their crests overgrown by the work of these zoophytes, which retained the form while altering the material of the surface exposed. On the other hand, the great superficial extent of some of the atolls and of their contained lagoons, remove from them all character of volcanic cones; and their close and peculiar arrangement in groups, like that of the Maldives, still further contradict the resemblance. The difficulty of explaining why such numerous detached summits should rise so closely to the same level near the surface, formed another obvious objection to the theory; and a more cogent one arose when it was discovered that the reef-building corals worked only to a limited depth, and could not, below this level, construct the coralline covering to the submarine peaks which the theory supposed.

Under the failure of these hypotheses, and pressed by other considerations, Mr. Darwin adopted what we have adverted to as the third solution of the question; viz. that the areas, greater or smaller, on which the coral reefs rest as a foundation - the flooring, in fact, of the seas - must have undergone progressive subsidence, so as utterly to withdraw islands or large tracts of land below the surface of the ocean; leaving the coral reefs which encircled or fringed their shores still on the water's level, and therefore under circumstances fitted to sustain their growth and position, even were the sinking of the foundation beneath them further continued. We must admit this hypothesis to be a bold and startling one, and such it appeared in the outset even to the most hardy of our geologists. Yet it has rapidly gained ground, not merely as the only one fulfilling the conditions required, but further because it well illustrates the different modifications and peculiarities of the coral reefs, and accords at the same time with certain remarkable discoveries which have distinguished the progress of modern geology. Mr. Darwin's 
application of his views in detail is characterised by great ability; but we can afford room only for a summary of the few leading points.

Look at the theory first in its application to the atoll, or simple coral islet with its interior lagoon. Instead of making this lagoon to represent a pre-existing cavity of the same form, as in the volcanic hypothesis, Mr. Darwin considers that it designates the place once occupied by a point of land more or less elevated, which has subsided downwards into the sea, leaving the coral reef circling round the centre, growing over its surface, and rising upwards by new constructions, where the subsidence has still continued. Let a mountain peak like Tahiti, girt round with a coral reef, sink downwarọ̉s from subsidence of the submarine area or other cause, and we should have the conditions just described taking place; and representing, by the various aspects of these islands, the stages of change from a lofty mountain to a few low points of land in the lagoon, and then to the simple coral islet barely rising out of the sea. Let the subsidence elsewhere be sudden instead of gradual - and we should find submerged atolls like the Chagos Bank; the power of replacement upwards being lost by the depth to which the surface has sunk below the sea.

Next, as to the linear reefs, such as the great barrier fronting the N. E. Australian continent. We have already spoken of the intimate relation of these to the reefs encircling islands; and we shall find the conditions of the theory applying to both in the same manner, and with equal probability. Suppose a prolonged line of reef to be built up on the shelf of the coast as it declines into the sea, leaving a narrow channel of water between. If the continental land gradually sinks, the line of coast will recede inwards, leaving a wider channel between it and the reef - the latter retaining its position, and being continually replaced upwards by fresh

$$
\text { c C }
$$


coral, as the lower portions of the mass subside. Sectional sketches would better illustrate these points ; but even without such aid, we think there will be no difficulty in conceiving them. The same views may further be applied to the other peculiarities of these formations, - the precipitous descent to vast depths on the outer side of the reef - the isles within the channel, forming residual points of the old continent - the fringing reefs - the openings into the channels or lagoons, \&c.

We have spoken of this as a bold hypothesis; and it will not appear less so when we look at the magnitudes, both of space and time, which are involved in such interpretation of the facts. The extent it is needful to assign to some of the areas of subsidence may well alarm an imagination not accustomed to deal with these subjects. Spaces of many hundred thousand square miles exist in the ocean, occupied by coral isles of such description as to admit of no other valid explanation than the sinking of the bottom of the sea over this extent. The length of the Australian barrier reef, 1,200 miles, proves that at least an equivalent line of coast has been subsiding since its formation began. The same inference extends to the great island of New Caledonia, in relation to the reef half encircling it. Mr. Darwin has delineated these several areas, as far as at present known, in a map prefixed to his work; adding to its value by designating also the areas of upheaval in the same oceans, and the sites of active volcanoes, interpreting by their proximity some of the remarkable phenomena in question. For both these great events - the subsidence and upheaval of the solid crust of the globe-are familiar to the speculations of modern geology, and variously attested in different parts of the globe by facts which, though recent in discovery, are unequivocal in the inferences they afford. The magnitude of these movements and changes may seem inconsistent with our ephemeral 
experience; but here, as in so many other cases, we are compelled to adopt new measures of time and space, when dealing with the physical conditions of the globe before man became a tenant of its surface.

If there be areas of upheaval as well as of subsidence in these coral seas, we may expect to find coral islands raised in places above the level of which these zoophytes effect their works. Accordingly, we have instances furnished by Captain Beechey, Mr. Jukes and others, of coral masses some hundred feet above the sea; with the same assurance of their having been raised from below, that we possess in the case of any tertiary stratum containing sea-shells. In connection with this topic, however, we must notice one objection to $\mathrm{Mr}$. Darwin's views which may seem to have some force, viz., that if masses of coral of such enormous thickness exist under the sea, we might fully expect to discover them in some situation or other among the great strata of the globe; knowing, as we do, how large a portion of these have been submarine in origin and raised afterwards into their present position. - Admitting the weight of the objection, that no such coral masses are found on our continents, we may qualify it by remarking, first, that we are not assured as to the relative period in the records of creation when the reef-building corals began their work in the seas; - secondly, that it is not impossible that some of the great oolitic, cretaceous, or other calcareous formations may actually represent coral deposits, formed as these are by the agglutination of various materials, and exposed for ages to physical conditions of which we can scarcely appreciate all the effect; - and thirdly, that the geological character of the lands in the coral oceans is still very imperfectly known, and we may yet discover such masses at greater elevation than any yet found, and exhibiting possibly gradations yet unsuspected into the character of the older calcareous rocks. 
Here therefore, as in all other branches of natural science, much has been added to our knowledge; while yet there remain many facts to be discovered and questions to be solved. In this the strength of science is shown, and not its weakness. It has futurity to work in, zeal for the work, and methods of research ever becoming more ample and more exact. Whether on land or sea, whether among the higher forms of life or the lowest, it is ever pursuing its straight and assured path towards a more perfect knowledge of the natural world. 


\section{LIFE OF DALTON - ATOMIC THEORY**}

[QUARTERLY REVIEW, No. 291.]

$W^{E}$ place these volumes in conjunction - the first a biography, the second an essay on one of the highest topics of natural science-because the fame of Dalton mainly rests on the discoveries by which he defined and illustrated that theory which forms the subject of Dr. Daubeny's work. A dedication of this second edition to the memory of Dalton - then recently deceased - justly and eloquently describes those researches in atomic chemistry which, while wonderfully enlarging the domains of the science, and giving exactitude to all its conclusions, have led to more profound views of the great laws by which matter is governed in the mutual actions and combinations of its ultimate component parts. Here, on this wide field of atomic theory, the bold

* 1. Memoirs of the Life and Scientific Researches of John Dalton, Hon. D.C.L. Oxford, LL.D. Edinburgh, F.R.S., Foreign Associate of the Academy of Sciences, Paris, \&c. By William Charles Henry, M.D., F.R.S. 8vo. London, 1854.

2. An Introduction to the Atomic Theory. By Charles Daubeny, M.I., F.R.S., \&c., Professor of Chemistry and of Botany in the University of Oxford. 2nd Edition. Oxford, 1851.

In the first Article of this volume I have spoken of the Atomic Theory and doctrine of definite proportions in some detail; but as one illustration only of the progress and spirit of physical science at large. The interest of the subject, however, is such that I trust to be excused for reprinting the present article; narrating the progress of the discovery, and the life of the man to whom it is chiefly due. It forms also an introduction to the succeeding article, on Modern Chemistry, to every part of which this discovery is so closely related. 
speculations of ancient philosophy had anticipated, as we shall presently see, some of the results, now better fixed by actual experiment and the singular refinements of modern analysis. Dalton had no knowledge of these elder hypotheses, nor even a full anticipation of all that his doctrine was to bring forth in the future. But it was he who in effect sowed the seeds for this great harvest; and though others had reached the very brink of the discovery, it was he who first fully indicated the principle and method of research, and the true import and value of the facts derived from it.

The name of Dalton must therefore enter into every history of the atomic theory; and we may be excused for dwelling upon some particulars of the life of this remarkable man, as afforded us in the volume of Dr. Henry, aided by our own personal recollections of him during a long: period of years. Apart indeed from his scientific career, it would be difficult to conceive an existence more calm and uneventful than that of Dalton. What Cuvier said of Cavendish is equally true applied to him - ' Il n'y a dans son histoire d'autres incidens que des découvertes.' Born in a humble position from which he only slowly emerged; - living successively in two provincial towns, where few at that time could understand or appreciate his labours ; - working always alone, with no other excitement than the love of physical truth; - wanting little, and undisturbed by the passions or even by the more common emotions of social existence-- his course was one of patient study, unbroken by any of the sterner incidents of life. He was a Quaker by birth, and maintained to the end the dress and many of the usages of the sect. But his character and habits depended much less on this than on his individual temperament, and those intellectual peculiarities of which we shall afterwards speak.

John Dalton was born at Eaglesfield, a village near Cockermouth, in 1766. The Daltons were of the class of 
small, proprietors, formerly called statesmen - a name that still lingers, we believe, in the northern parts of Engiand. His father appears to have been a weaver as well as yeoman; but of slender means in both capacities. He had two sons and a daughter. John, the second one, was placed at the village schools at Eaglesfield and in the neighbourhood; but derived more aid from the talents with which he was born than from any help which such schools could give. He speedily nurtured his own faculties into activity, and the slight memorials of his youth are the miniature of the man in later life. This miniature becomes more exact as we follow him forward to his early positions in the world; first, as a schoolmaster himself at Eaglesfield, when only twelve years old; - next, as assistant and principal successively, at a boarding school at Kendal. Simple as were, and still are, the functions of a village schoolmaster, it is extraordinary that a boy of twelve years should be able to fulfill them; and that, after a year of intermediate labour in husbandry, he should have been called, when yet but fifteen, to the larger duties of the Kendal school. That inborn faculty of silent self-labour and patient study, which remained with him through life, can alone explain this.

Some of the moral peculiarities of Dalton's character come out curiously in this part of his career, as derived from the recollections of one or two persons yet living, who were his scholars at Kendal. Apart indeed from such information, we could readily have conjectured that he must have made a very indifferent schoolmaster. His own early self-acquired knowledge did not give him the power of instilling the same into others of his age. At no period had he any command of language or facility of explanation. Equally was he unfitted to comprehend those mental diversities of temper as well as intellect, which show themselves in the very dawn of life. Whether that uniformity of plan, which 
is in some sort inevitable in the gathering together of youth in schools and colleges, be not on the whole better in result than the teaching upon vague views of individual character, is a question we cannot here discuss. It is enough to say that Dalton, as a schoolmaster, could have had but one method, and that founded on his own peculiar temperament and habits.

At the Kendal school, where there were some sixty boys and girls educated at from ten to fifteen shillings a quarter, he was associated, while master, with his brother Jonathan, a hard and severe man by nature. The surviving pupils describe John as of gentler temper; but nevertheless cold, abstracted, and uncouth in his ways. The school, at best, seems never to have been very popular under the management of these two young brothers.

While residing at Kendal John Dalton engaged himself in frequent contributions to the 'Gentlemen's and Ladies' Diaries ;' two periodical works which, at that time of scanty literature in the country parts of England, earned repute and circulation by their prize questions in mathematics and general philosophy. When Westmoreland was some days' journey from London, instead of the eight hours of present travel, such periodicals, with a weekly newspaper circulated among neighbours, were probably treasured more than the superfluity of publications now spread throughout every corner of the kingdom. In 1787 we find that Dalton, being then twenty-one, correctly solved thirteen out of fifteen mathematical questions in these diaries; and in 1790 gained the highest prize for his 'masterly solution of the prize question.' He meddled a little also with the moral queries propounded in these works; and his answers, though somewhat formal and vapid, are at least as good as the questions deserve.

Dalton began his career of physical research while at 
Kendal, directing it chiefly to Meteorology - a subject which engaged much of his attention through life. The first entry in his Meteorological Journal is of March 24, 1787, and records a remarkable Aurora Borealis on the evening of that day. Perchance from this very cause the phenomenon of the aurora (even now imperfectly explained) continued ever after to be a favourite topic with him. He made in the beginning his own barometer and thermometer; and used as an hygrometer some six yards of whipcord, suspended from a nail and stretched by a weight, with a scale attached to it. This rudeness of his instruments was not limited to early life. Even in the experiments which led to his great discoveries, his apparatus was grievously deficient in those refinements which Chemistry now requires and has fully obtained. His laboratory, which we once visited, might well in its slovenly arrangements provoke a smile from the modern adept in analysis. There was a sort of obstinacy in Dalton's mind on this subject; derived in part from the independence of his own early labours - in part also from an original pertinacity of his nature. But some compensation was found for this defect in his clear perception of the objects sought for, and in that patient repetition of experiments and observations which reconciles discordant results, and gives certainty to the conclusions obtained. The Method of Averages, even where not recognised as such, involves a principle prolific of truth; and Dalton largely availed himself of it in his labours.

In May 1792, he first visited London, of which he says in a letter to his brother, 'It is a most surprising place, and well worth one's while to see once; but the most disagreeable place on earth for one of a contemplative turn to reside in constantly.' A longer knowledge might, perhaps, have told him that a man may be alone in a multitude; and that the greatest works of contemplation as well as of practical activity have emerged from amidst the din and bustle of this 
great metropolis. It is a characteristic trait of him, that he occupied himself while going to the Friends' Meeting-House in counting the number of carriages he met on the road. 'This,' he says, 'I executed with tolerable precision, and the number was 104.' Dalton lived, in truth, in an atmosphere of numbers; and all his thoughts and observations took their colouring from this strong propensity of his nature.

In 1793 he first published his Meteorological Observations and Essays, in which he records his obligations to Mr. John Gough of Kendal; that singular man, who, becoming totally blind from small-pox when two years old, furnished a memorable instance of what the intellect can attain, unaided by this one great sense. Profoundly versed in mathematics, he became familiar also with every branch of natural philosophy; and had so cultivated his remaining senses that he could tell by touch, smell, or taste, almost every plant within twenty miles of his native place. Dalton's friendship for him continued throughout the whole of Mr. Gough's long life.

It was in the same year, 1793, that Dalton made his second and final change of residence, by accepting the place of mathematical tutor at a College of Protestant Dissenters lately established in Manchester. Though his connections with the College ceased after six years, he remained at Manchester during the rest of his life, and in the same house for the last thirty years of that time; making an income which sufficed for his few and simple wants by giving lessons to pupils or by occasional lectures; both at a very low rate of remuneration.

We suppose that few men of tolerable education have passed through life without putting together some lines, which either were poetry, or were believed by themselves to be such. Among the exceptions to the rule we should fully have expected Dalton to be one. But it was otherwise. His biographer gives us, as the best among other specimens, ten 
or twelve stanzas, addressed to an Folian lyre; and written in 1796, at a time when his feelings seem to have been somewhat excited by the beauty and talents of a young Quaker lady, whose family he occasionally visited at Lancaster. In letters to his brother he describes these qualities with more warmth and in greater detail than we should have expected; yet still with a certain philosophical method and a strong leaning to the 'tabular form,' which delineate the man almost as well as the lady whom he admires. With regard to the verses, they surprise us from being very much in the Della Crusca style; and as poetry we can hardly commend them. Yet we give a stanza below, which will not be thought deficient either in harmony or feeling. In reading it we have a difficulty in recognising either the Quaker or the hard dry mathematician of the Kendal school.*

Whatever might be the state of Dalton s feelings at this time, result there was none. The same condition of life continued; one which probably made marriage impossible, even had he not been already wedded to those very different pursuits which gave happiness as well as honour to his life. It was about this year, 1796, that Chemistry first engaged his attention; and as a Member of the Manchester Literary and Philosophical Institution (of which he afterwards became President) he placed before them in successive years a series of papers of great value in connection with this and other branches of Natural Philosophy; evin'ing both the extent of his objects and the energetic and successful labour he bestowed upon them. Without enumerating these different Memoirs, we may say generally that the most important of them relate to the weight, temperature, and moisture of the atmosphere;

* Again the slowly rising notes assail -

As if some tender maid, unseen, unknown,

Sigh'd for neglect - yet tuneful swell'd the gale,

To melt th' unfeeling heart with sorrow's plaintive moan. 
- to the various phenomena of heat and cold, as produced by or determining the state of other bodies; - and to the constitution of mixed gases, and especially of those forming our atmosphere. Later observations and experiments, less rude than those which he employed, have tended to invalidate some of his conclusions. But many yet remain untouched; - all have contributed to the advancement of science; and all give eminent proof of his sagacity and boldness of research.

We do not here follow the labours or writings of Dalton into the great discovery of his life, as we shall speak of this hereafter. Before it was sufficiently matured for publication, he was invited early in 1804 to give a course of Lectures on Natural Philosophy at the Royal Institution in London. With some kind and valuable aid from Davy, he appears to have gone through the course satisfactorily; and, from his own account, with a good deal of applause. We cannot but think, however, that he unconsciously overrated his success; and that what he considered such was but a courteous regard of his hearers to the simplicity and peculiarities of the man. A few years later, when his discoveries were still only partially. known, we heard him deliver a short course on this subject at Edinburgh to a scanty audience. It would be hard to conceive anything more uncouth or ineffective than his manner of dealing with the great physical truths that lay before him. His experiments, as made in public, frequently failed. His voice was harsh, indistinct, and unemphatical; and he was singularly wanting in the language and power of illustration needful to a lecturer on these high matters of philosophy, and by which Davy and Faraday have given such lustre to their great discoveries. Among other instances of his odd appropriation of epithets, we recollect that in treating of oxygen, hydrogen, nitrogen, \&c. - those great elements which pervade all 
nature - he generally spoke of them as 'these articles;' describing their qualities with far less earnestness than a London linendraper would show in commending the very different articles which lie on his shelves.

Dalton's doctrines therefore needed other advocacy than his own to bring them fairly before the world. Nor was this aid wanting. We shall have to mention soon the names of those eminent Chemists who speedily recognised the value of the discovery, and sought by their labours to verify and extend it. His own life proceeded meanwhile in the same course of tranquil labour. He was now, however, less occupied with new objects than with the completion of his previous researches, and the removal of objections which had been raised to certain parts of them. With all his love of truth, in science as in other things, Dalton was strongly tenacious of conclusions once formed; and there were many opinions to which he clung, long after more exact experiments than his own had shown them to be doubtful or inadmissible. We may name as instances, his obstinate adherence to the atomic weights he first assigned, though proved to be incorrect; - his reluctance to adopt the doctrine of volumes, received by all other chemists; - and his long struggle against the recognition of chlorine as a chemical element.

Though always recluse in his habits, his reputation, now established both at home and abroad, inevitably enlarged his intercourse with the world. Manchester too (at least those in it who found leisure for anything beyond the labours and profits of the loom) began to feel pride in the fame of their fellow-townsman. But his private life was little changed in its simplicity - his character not at all. While streets, factories, and steam-engines were growing up multitudinously around, he continued to reside in the same quiet house and family in which he finally closed his career. He seldom went into what is called society. His associates were chiefly 
those who had pursuits akin to his own; and a few intimate friends and pupils, with whom he sometimes unbent himself after the labours of the day. One of these friends describes him as exceedingly cheerful and facetious on such occasions. We can readily believe in his cheerfulness, but find it difficult to imagine in Dalton anything approaching to wit. Even in the fashion of his amusements there was the same order and method as in other things. Every Thursday afternoon he indulged in his favourite diversion of bowling; watching with a sort of scientific eagerness the motions of the bowls, and noting down minutely in a book all the losses or gains of the few pence for which the game was played. In every other habit of his life, as to hours, labour, and food, there was the same simplicity, order, and temperance.

Occasionally he visited London, or made excursions into the Lake scenery of his native county. A notable incident in a life thus tranquil was a visit to Paris in 1822 , which we know to have afforded him singular pleasure. Six years before he had been elected a Corresponding Member of the Academy of Sciences - a proof of the reputation he had already earned abroad. Mr. Dockray, his companion on this journey, graphically describes a dinner given to Dalton by Laplace at his country-seat at Arcueil; the beautiful grounds of which derive no less fame from having been the residence of this philosopher, than from the earlier destiny of the spot as the abode of the Emperor Julian, when Governor of Gaul. At this dinner Berthollet, Arago, Cuvier, Biot, and Fourier were among the guests - names all illustrious in the annals of science. Mr. Dockray pictures to us Dalton walking in the evening through the ruins which yet remain of Julian's residence, between Laplace and Berthollet - a remarkable group, and almost as much so in the diversity of the men as in the community of their fame. From our own recollection of Laplace we feel how great 
must have been the contrast between him and Dalton; - the latter what we have already described him - the former equally placid in demeanour, but with the air, habits, and courtesies of an old French nobleman. In estimating their relative genius we must needs rank Laplace far above the level of Dalton. Both of them mathematicians, they yet trod in mathematical paths so remote from each other as almost to efface every vestige of this connection. The very diversity here is the exponent of the scientific character of the two men. Dalton may be said to have worked in straight lines, both in mathematics and general physics; with definite objects placed clearly before his conceptions, which he pursued steadily by the simplest or even rudest methods, to the attainment of the results desired. The genius of Laplace took for its sphere of action the wide domain of the universe : and while by the mighty power of his analysis he was removing anomalies, and reconciling even the secular perturbations of the planets to the one great law of gravitation, he applied the same power and the same methods of evidence to almost every part of human knowledge. $\mathrm{He}$ worked not in straight lines but sweeping over a vast circle, and bringing each part into relation with the whole.

It is difficult to give any single definition of genius, having due regard to the endless varieties and anomalies which present themselves in the human intellect. In the higher acceptation of the term, Dalton could hardly be called a man of genius. He had not those wings with which some men soar over the ocean of undiscovered truth, discovering regions to be submitted hereafter to human intelligence and power. But he brought to his researches, as we have seen, the habits of a sagacious and intrepid thinker; swayed by no authority but that of facts, and sedulous in seeking for these by his own labours and methods. We believe this description to include all that is most peculiar in his character as a philosopher. 
Dalton's connection with the French Academy was not limited to his first honour in it. In 1830, on the death of Sir H. Davy, he was elected to fill his place as one of the eight Foreign Associates of the Academy; a distinction, from its rarity, fitly regarded as among the highest which science can bestow. It is remarkable that until 1822 he was not elected a Fellow of the Royal Society. The absence of all ambition or effort on his part must be received as the cause of this delay. In 1826 his high merits as a discoverer were fully recognised by the Society in the award to him of the first of the royal prizes given after their institution.

Honours indeed now began to fall more thickly upon him. The establishment of the British Association in 1831, the annual meetings of which Dalton repeatedly attended, was in some part concerned in this effect. Though his discoveries were at this time fully established and familiar to the scientific world, the seclusion of his life had permitted few to know him personally; and the Quaker philosopher, now well advanced in years, stood among his brethren in science at these meetings, a new object of attention and interest. The simplicity of his demeanour, from which age had taken off its earlier uncouthness, won upon the feelings of all; and even at later meetings of the Association, when the novelty had worn off, Dalton was ever regarded with affectionate veneration.

In 1832, at the meeting of the Association at Oxford, the honorary degree of D.C.L. was conferred upon him, in conjunction with Faraday, Brewster, and Robert Brown. The same honour he received at Edinburgh two years later. In 1833 under Lord Grey's government, a pension of $150 l$. a year was settled upon him, providing a happy release from the burden of elementary teaching. About the same time the people of Manchester subscribed 2,000l. for the statue of a man who thus honoured their town; and its execution was 
fitly committed to Chantrey, whose genius rendered it a wonderful work. While Dalton was in London sitting to this great sculptor, it was suggested by his friend, Mr. Babbage, that he should be presented at the King's levee. His own acquiescence being obtained, the preliminaries of his dress as an Oxford Doctor of Laws settled, and preparatory instructions given by enacting the levee in a private room, he was presented to William IV., who seems to have questioned him with the kind familiarity which belonged to that sovereign's nature. Mr. Babbage, present with him on this occasion, heard one court officer say to another, 'Who the $d-1$ is the fellow whom the King keeps talking to so long?' This gentleman would have been still more surprised had he seen the Quaker garb concealed under the scarlet robe of the University of Oxford.

Dalton's life was continued ten years beyond this time, to the age of 78 ; but we have little more to record. The last few years formed a period of gradual but sensible decay in his faculties both of mind and body, consequent upon a paralytic seizure in 1837 , followed in 1838 by a slighter attack of the same nature. He did not die until 1844; but the antecedents of the final change were on him before, and, for some time at least, consciously so to himself. In 1840 he presented a paper to the Royal Society on the phosphates and arseniates; so obscure throughout, and the conclusions so erroneous, that the Council declined its publication in the Transactions. Dalton, much mortified, printed it separately with the indignant comment annexed to it - 'Cavendish, Davy, Wollaston, and Gilbert are no more.' Even after this, however, he published four short chemical essays; but these were probably the result of prior labours, since the last two contain the elements of a discovery of so much interest, that he himself says, "It is the greatest discovery that I know of, next to the atomic theory.' We may briefly denote the 
subject, by stating that it was that curious research into the relative conditions of the water and solid parts of hydrated salts, which has since been so admirably prosecuted by Playfair and Joule; verifying in the main, while modifying in parts, the remarkable results obtained by Dalton's sagacity. We do not find anything to tell us at what period the latter was engaged in this enquiry; but doubtless some time before that impairment of his mental powers to which we have just alluded.

At Dalton's age a change of this nature, and evidently connected with cerebral disease, is insuperable by remedies and sure to be augmented by time. Though appointed a vice-president at the meeting of the British Association at Manchester, 1842, it was merely as a tribute to his name. In May, 1844, another fit occurred; and on the 27th of July he fell out of bed and was found lifeless on the floor. A post-mortem examination showed no recent rupture of any blood-vessel, but great venous congestion; and in one part of the brain, distinct traces of the extravasation which had probably produced the first paralytic attack. There was too much of ostentation in all that followed the event. His coffin, placed in public in the Town Hall, was visited by more than 40,000 spectators, and a procession of nearly 100 carriages followed it to the grave. This was scarcely in accordance with the life and character of the man.

The portrait of Dalton, prefixed to Dr. Henry's volume and taken from Chantrey's bust, faithfully represents his countenance, and especially what we would call the austere gentleness of his expression. His was a face which told the whole character at once; - its earnestness, simplicity, and truth; and even that power of patient methodical labour, which marked and determined every step in his career.

We have very little clue to Dalton's religious opinions. $\mathrm{He}$ was scarcely ever heard to allude to the subject; and 
though he attended twice every Sunday at the Friends' Meeting-house, he never took other than a silent part in their devotional exercises. When such was his reserve upon this point, even to those most intimate with him in life, we have no right to hazard suppositions of our own which can never be verified, and which might perchance be widely removed from the truth.

After this short sketch of Dalton's life and personal character, we have still to speak of the discovery which gives the greatest lustre to his name; of its connection with prior systems or theories; and of the influence it has had on the subsequent progress and direction of physical enquiry. On several of these points, however, we have dwelt so far in preceding articles, that we shall now limit ourselves chiefly to what may be called the history of the Atomic doctrine, as it comes down from antiquity to our own time. This, too, must be a mere sketch; but it has interest, not solely as a part of science, but as a picture of the human intellect in its progress towards truth.

Such history, however, is not without its difficulties. In discussing the atomic theory, we often touch on that debateable ground between mathematics and metaphysics, which D'Alembert has well named ' l'abîme des incertitudes;' there being scarcely a step in the argument which does not approach in some point or other to this boundary of human intelligence. While modern science is defining by strict numerical formulæ the proportions in which the molecular combinations of bodies take place - and often with such certainty that the chemist can foretell the results of an analysis before the labours of the laboratory have begun the demonstration of facts thus obtained is at every step urging the mind towards those unseen properties and profound laws of the material world, where thought is forced to 
pause and further demonstration is lost in darkness. There may be rashness indeed in marking too absolutely any line of demarcation, for the science of our own day has often swept over what seemed to be the final limits of our knowledge, and carried its adventure into open fields beyond. The subject before us, moreover, is one where physical evidences still crowd in from so many separate and unexpected sources, that it is hard to curb the efforts of the mind to theorise upon the results already obtained.

This very multiplication of proofs is in itself a hindrance to a concise and popular view of the modern atomic doctrine. The physical evidence is not merely various in its sources, but presents peculiar modifications, adding much to its complexity. The recent discoveries in Isomorphism, Isomerism, and Allotropy (names in themselves formidable to the ear), indicating some of the various modes in which the molecules of matter, simple or compound, are related to or act upon one another, - all bear essentially on the question of the atomical constitution of bodies; and require an interpretation accordant at least, if not common to all. An elementary view of the subject is further embarrassed by the anomalous or doubtful points which still remain for solution; - such as the case of a numerical series, perfect in its other parts, being stopped by an anomalous fiaction, only to be removed by such better analysis as may bring the refractory element into the scale from which it seemingly departs, or by altering the first term so as to convert the fraction into a simple number in the series.

The statement of these difficulties is not altogether without its use. It suggests, first, the demarcation, as far as this can be drawn, between the metaphysical and physical parts of the atomic philosophy;- between that which is purely or chiefly speculative, and that which depends on experiment and is expressed by formulæ of numbers. And secondly, in 
regard to the latter class of researches, it indicates the purport and scope of the enquiry, as embracing all those relations of bodies which depend on the numerical proportions, arrangement, or mutual substitution of the atoms severally composing them.

Following then this suggestion as to order, the speculative part of the atomic philosophy first comes before us. In every age and community there are certain minds prone, from their very constitution, to an earnest scrutiny of the world around them. It is an instinctive and almost compulsory use of one of those powers committed to man by his Maker; the variety of which powers in different men seems as much the object of a wise design as their original bestowal. The mere existence of Matter in its relation to Spirit, and the endless forms and changes which material bodies assume, whether subordinate to human will or not, irresistibly excite such minds to some form of speculation, however vague in method or conclusion. We may omit here all notice of those crude notions of earlier ages and ruder races on this subject, which have partially come down to us; but we must pause awhile amidst the riper speculations on the elementary qualities of matter, which the philosophers and poets of Greece and Rome have transmitted to later ages. To the Greeks more especially we owe those theories, at once subtle and bold, which, while they seize a portion of truth by a sort of intuitive perception, do yet neglect to fortify this by experiment or exact observation. It is hard to explain how a people, not merely subtle and inventive, but capable of high thoughts and philosophy, and keenly inquisitive into the secrets of nature, should never have been fairly imbued with the principle and methods of experimental research. The exceptional cases are rare, and rather augment than lessen our wonder at the fact. It is surprising that the success of particular experiments and inductions should not have led 
the way to other like researches, even had the principle of enquiry not been suggested by the phenomena of the world around them.

However this may be, the absence of anything like analysis limited the Greek philosophy to purely speculative doctrines regarding matter, and the various concourse and combinations of atoms to which its forms and qualities are due. Of these theories the volume of Professor Daubeny now before us (unpretending in form, but of great merit in execution) - gives a clear and sufficient account. Dr. Daubeny brings indeed high qualifications to his work; a philosophical spirit, classical knowledge, and an intimate acquaintance with the doctrines and discoveries of modern Chemistry. All are required for the complete view of a subject of such wide compass and singular complexity.

The first great problem belonging to it - one which has engaged the attention of thinking men in all ages - is the origin and nature of Matter, as distinguished from mind or Spirit, and also from that notion of the void in space which has ever entered into the enquiry. The genius of the Greek philosophy dealt with this question in its most abstruse forms; but not before such speculations had already found place in the philosophical, religious, or popular dogmas of yet earlier and more remote Oriental races, to whose mental temperament they seem to have been singularly congenial. Stripping off the various dresis and disguises of language, they are in fact the self-same questions which have descended to our own time; and which we are destined to transmit (still unresolved, though better defined) to our own philosophical posterity. Unaided human reason, indeed, under whatever form of words or logic it may shelter its weakness, must ever be baffled by such questions as, - Whether matter has any existence apart from the perceptions of the intellectual being? - Whether it is eternal in itself, and moulded only by 
the acts of creation, or actually brought into existence by the power which has thus moulded it? - Whether, if eternal, its nature be not such as to limit and constrain this power, which has framed from it the order of things we see around us ?* Wonderful we may well deem it that man should be gifted with a spirit able to propound these and other like subtleties to itself. But true philosophy consists in setting a boundary between these vague impracticabilities, and that great field of observation and experiment which the Creator has privileged man to work in, by giving him faculties fitted for this wiser and better labour.

All that can rightly be called atomic philosophy - the investigation of matter in its molecular parts, and under the different combinations and mutual actions of these - comes distinctly within this field of legitimate enquiry. Yet here, too, rash speculation had a long period of supremacy. We have already alluded to those hypotheses of the Greek philosophers, through which, unaided by experiment, they sought to explain the multiform shapes, combinations, and changes which matter assumes or undergoes. They saw, as it was easy to see, that for such an explanation it must be supposed divisible into parts of exquisite minuteness: since under no other conception than this are the phenomena of possible fulfillment. It was further seen (and almost by the same necessity) that these minute parts, molecules, or atoms, must have definite relations, whether of attraction or repulsion, to one another. All nature, animate or inanimate, teems with evidence to this effect, and no experiment was needed to attest it. The conception of definite proportions in their molecular relations - now ripened into a great

* Other speculators again, more purely Oriental in character, assign to matter an actual inherent malignity of nature, opposing itself to what is good in creation, and thence bringing evil into the world. The doctrines of the Chaldeans, of Zoroaster, and of the Indian mythologies, are all based more or less on this conception. 
physical law - can hardly be said to occur in the ancient philosophy though some few passages may vaguely express the idea. But another question, yet current in our own time, and which lies at the bottom of every atomic theory, engaged more distinctly the notice of these schools of antiquity - a question which neither reason nor experiment can ever do more than settle presumptively - viz. whether there are truly ultimate molecules or atoms of matter insusceptible of further division? or whether we must here, as elsewhere in nature, veil our reason before that metaphysical infinite which baffles alike definition and conception, and consider matter as divisible without limit or end?

The most rational of the Greek philosophers settled this abstruse question in the same way in which it has been conditionally determined by the sounder methods of modern

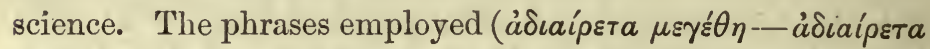

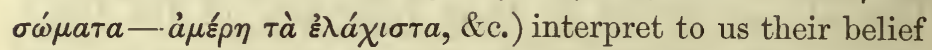
that there are portions of matter, inconceivably minute, which are absolute units in themselves, and admit of no division beyond. This doctrine is wholly distinct from the ancient theory of the four elements (the 'four champions fierce' of Milton), which served as a coarse formula for reason to work upon, before it had been shown by experiment how completely these elements are mutually convertible throughout every part of the material world.

Another point in the ancient atomic philosophy, anticipating modern enquiry though running beyond it, is the question as to the form or configuration of those indivisible atoms, which may be regarded as the true units of the material world. Here, again, all was mere speculation; and the geometrical forms assigned to different conditions of matter were generally based on the feeblest and most frivolous analogies. Whether modern science, with its more severe and scrupulous enquiry, can ever attain to more perfect proof 
is yet hidden in the future. Certain paths, which we shall hereafter notice, seem opening in this direction and have been zealously pursued. We live in an age of new methods, as well as new facts; and where the object is not in its nature insuperable by human reason, we may venture to aspire, if not to certain truth, at least to that degree of presumptive proof which is proximate to it.

In the foregoing outline of the earlier questions and opinions as to the atomic constitution of matter, we have refrained from naming those of the ancient philosophers to whom we chiefly owe them, believing that we might thereby convey a clearer view of their purport and success. It will occur to others as to ourselves, that the history of human knowledge is often more clouded than illustrated by the long array of names and shades of opinion, which are made to enter into it. In the personal narrative of these victories or vagaries of human thought, the reader loses sight of the questions which lie at the bottom of the whole, and fails of discerning what has really been done or is still left undone. We cannot, however, omit to name some of those who early laboured on this subject, seeing the eminent place they hold in the history of mankind.

It was impossible, indeed, that such men as Pythagoras, Plato, Aristotle, Democritus, Empedocles, and Epicurus for these names stand on the list-should live in a world full of such strange problems, without being attracted forcibly to them. The principle of numbers as propounded by Pythagoras, though extravagant in its mystical applications, yet is in some sort an anticipation of what now rests on a close induction of facts. Leucippus has been vaguely recorded as the first who treated of the atomical composition of matter. Democritus and Epicurus are with more certainty reputed as the philosophers who gave higher form and consistency to the theory; including under 
it the various questions to which we have already adverted. We are far from adopting Bolingbroke's phrase of the 'lofty madness' of Plato, but must nevertheless admit that his doctrines respecting matter are metaphysical without any distinct meaning. Aristotle brought to the subject a more practical understanding and a larger observation of nature, yet added little to our real knowledge. His $\pi \rho \omega ́ \tau \eta ~ \tilde{~} \lambda \eta$, or primitive matter, is contrasted with the vov̂s, or intelligence, which brings it into form and action. He seeks, through the doctrine of the four elementary properties, to explain the various conditions which matter assumes; and, misguided by that notion - to which the Greek philosophy ever leant - of opposite and neutralising qualities, he failed to perceive the simpler and truer induction, that heat and cold, dryness and moisture, are not conflicting elements, but different degrees of the same quality or state. He compromises the question as to the infinite divisibility of matter, by admitting that atoms may be actually indivisible, though not potentially so; - a distinction of a scholastic kind, and aiding little towards the solution of the question.*

We pass over a long period to come at once to Lucretius, as the expounder in verse of the doctrine by which Epicurus gave more perfect system to the atomic theory; extending and modifying the views of Democritus and others who had gone before. A few lines, however, must suffice us for the record of this system, which many of our readers may already know in part through the great work of the Roman poet. It represents the space or void of the universe as penetrated and traversed in every direction by tides or currents of elementary indivisible atoms - infinitely minute, but possessing gravity - of various figure or shape, but these unchangeable for each - having an intrinsic power of motion, and unceas-

* We quote the words of Aristotle, as characteristic of the style of this great

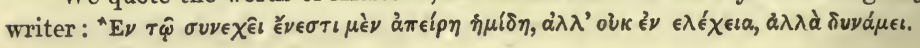


ingly permeating every part of space with inconceivable swiftness. From the fortuitous concourse, collision, and adaptation of these atoms, thus eternally in motion, the material world is formed, and the various compound bodies upon it are successively generated, changed, or renewed. The seeming attribution of creative power to the self-organising nature of the atoms themselves, and the negative condition assigned to the gods by Lucretius, have drawn down the reproach of atheism on this system. It may more justly perhaps be called an abandonment of the popular mythology of the age in which the poet lived.

This is the outline of the doctrine. The details, whether furnished by philosophy or poetry, we have little room to dwell upon. It has not been the fortune of any other philosophical hypothesis (unless we suppose an exception in the lost writings of Empedocles) to be thus 'married to immortal verse.' It may be alleged that the greatness of the poem of Lucretius was not so estimated by his contemporaries, and we admit the fact; but attribute it chiefly to the nature of his subject, less congenial to the mind of Rome than to that of Greece, and which even Cicero scantily admits within the pale of his philosophy. Still we confess our surprise to find in Ovid the only adequate acknowledgement of the grandeur of Lucretius as a poet; and that Quintilian, a consummate critic, should notice him in terms of such bald and languid commendation.

Quitting this sketch of the ancient atomic doctrines, and passing over, as almost null, all that the mediæval philosophy and even the Arabian chemistry produced, we proceed to the later opinions sanctioned by the great names of Descartes, Newton, Leibnitz, and Boscovich. Newton, attributing to God the first creation of primitive units or particles of matter, describes their endowments in terms not unlike those which Lucretius applies to his self-acting atoms. Leibnitz, resting 
somewhat dogmatically upon his two laws of Continuity and Sufficient Reason, rejected this notion of solid primitive atoms; and arguing, as Descartes had done, that no body can be admitted as indivisible, sought to supply their place by an hypothesis of monads, or points without extension. The more celebrated dynamic theory of Boscovich is a modification of this view; in which, for the action of material particles, is substituted the idea of simple centres of force, that is, points of attraction and repulsion. Though this view has gained some favour of late, we cannot find in it more than a new mode of expressing the limit of our knowledge. And the expression is faulty in itself, inasmuch as the term force can only be intelligible where there is something which is the subject of it; and attraction and repulsion are without meaning, unless there is something more than unextended points to be attracted or repelled. Boscovich vindicated his doctrine with ingenuity; but we doubt whether it has rendered, or can ever render, any real aid towards the solution of this great physical problem.

We now come with satisfaction to those more recent researches, which, based on experiment, have given to this subject all the higher characters of an exact science. We have seen that the most complete of earlier systems scarcely went beyond the fortuitous concourse of atoms as the cause of all existing things. It is the pride of our time to have changed chance into certainty; - to have submitted to numerical formulæ the various relations of material bodies; - and to have framed a system of definite proportions, perfect enough to allow the prediction of the unknown from that which has been already discovered. In fine, it has belonged to the progress of this part of science (as, in truth, of every other) to put aside all accident from the creation of what we see around us; and to give proof and certainty to those great 
laws designed by a Creator whose wisdom and power we recognise in all their wonderful results.

Experimental Chemistry is the portal through which access has been found to this new region of enquiry. A few considerations will make this clear to our readers; and it is important to the understanding of the subject that it should be so. Whatever reasons might be afforded by the obvious changes of material bodies for the atomic theory of their constitution, no absolute proof could be derived from such phenomena. It was a strong presumption of the existence of minute, perhaps indivisible, molecules; but did not go beyond this. The discovery of fixed multiple relations of weight or volume in all chemical combinations, was the fact which at once substituted a science for a mere probability; and converted an hypothesis, barren of results, into a system and law of nature, fertile beyond any other in its consequences and conclusions.

We would especially dwell on the effects produced by the admission of weight, as a principle and instrument in this research. The fate of Chemistry, as the science mainly concerned, may be said to have hung upon the balance. We are now so accustomed to the method of numbers and weights, that it is not easy to recognise this science as ever existing without it. Yet such in great measure was the fact. To Lavoisier, almost a contemporary, we mainly owe the introduction of the principle and its right application; - at once subverting theories like that of Phlogiston, which had grown up without this correction, and laying the foundations of new and more certain knowledge. It is easy to discover in this change the basis of the doctrine of definite proportions. If in the relations of bodies to one another we find the weight of those entering into combination, or separating from it, to be absolutely and invariably the same for each, the principle is already obtained, and prepared for extension to 
the innumerable cases which Chemistry puts before us. We are the more earnest in pressing these general views, inasmuch as they help us to comprehend the whole history of this part of science. It is obvious that the processes of an imperfect analysis could never substantiate doctrines which have the numerical proportions of weight for their foundation. It is the increasing exactness of analytical chemistry which has furnished a basis for that new theory which we now have under review.

Certain questions as to priority of discovery meet us here, even in the outset. This, it is well known, has happened in almost every similar case; - the result sometimes of accident, sometimes the effect of a simultaneous direction of the labour and genius of many to objects already indicated for research. In the history of the greatest discoveries - even that of universal gravitation - we find the record of men who have seen the light before them, have approached near to it, but have missed the sole path by which the lamp could be seized. But the example which most bears on our present subject is the controversy, actively revived of late, as to the discovery of the composition of water - the greatest single step ever made in chemical science. We do not enter on the question here; but merely cite it as a striking instance of that concatenation by which the labours of men of genius are blended together for the advancement of knowledge and the good of mankind.

The closest anticipations of Dalton's discovery are doubtless to be found in the researches of Wenzel and Richter, two German chemists, and of Mr. Higgins of Dublin. Wenzel led the way, now nearly eighty years ago, by the execution of very exact analyses of neutral salts, which gave proof that when two such salts decompose one another, the compounds thence resulting are precisely neutral also. The two bases and two acids are exchanged in proportions exactly 
equivalent; and two new salts are formed, without either loss or addition in the act of change. A little consideration will show that this single fact, well attested, discloses the whole principle of definite proportions; and the possibility by a simple calculation of making a few analyses of such neutral salts the key to others yet unmade. This principle was taken up fifteen years later by Richter; who verified the facts, and gave a tabular form to the reciprocal proportions or equivalents thus obtained.

The researches of Mr. Higgins, first published in 1791, make a still closer approximation to those of Dalton, and may seem to justify the claim of priority; - put forward by himself when the subject was yet fresh before the world, and since urged by others on his behalf. There can be no doubt that Mr. Higgins announced thus early, as the result of his enquiries, certain conclusions which tally closely with those obtained by Dalton;-as, for instance, that a molecule of water is composed of one particle of oxygen united to one of hydrogen; - that the five chemical compounds of oxygen and nitrogen vary in the multiple proportions of oxygen added to the single particle of nitrogen; - that sulphurous acid contains one particle of sulphur with one of oxygen, sulphuric acid two particles of the latter element. These conclusions, since verified, are doubtless very remarkable; the more so as they seem to have been derived from imperfect data, and without any clear conception of the great consequences they involve. But whatever rank Mr. Higgins may finally take as a discoverer, his researches fell at the time upon a heedless world. We have before us conclusive evidence that Dalton was ignorant of them until long after he had matured his own doctrines. He read little; - thought and worked alone in his laboratory; - and was too upright not to avow what he got from another. His sery nature made it impossible for him to be a plagiarist. 
We may further remark that even had the labours of Mr. Higgins been known to him, he still must be accounted the discoverer of the general law of multiple proportions, of their connection with the relative weights of combining atoms, and of many of the methods by which this great principle is verified and made the foundation of practical chemistry. These are the points upon which Dalton's fame will indisputably rest with posterity.

He has himself furnished few materials for marking the successive steps of his research. We are told of Kepler that he tried eighteen hypotheses as to the orbit of Mars, before reaching the discovery of the true one. Dalton was led by the course and results of his earlier labours, to conceive the power of subjecting to numerical forms the endless and seemingly capricious varieties of chemical phenomena. We know not precisely where the light first broke in upon him; - where, perchance, it was again obscured for a time by the imperfection of the analyses upon which his doctrine depended; - or where, finally, he saw before him the full day of the discovery. The main facts appear to be, that he derived his earliest conception of the law of multiple proportions from his experiments on gases, or matter in the aeriform state; - that in a paper read to the Manchester Society, Nov. 12, 1802, he announced the first example of this law in the combinations of oxygen and nitrous gas; - that the atomic view of chemical combinations occurred to him in direct sequel to, if not simultaneously with, the establishment of the law; that in October, 1803, he read another paper to the Society, in which, after stating that he believes the enquiry to be entirely new, he assigns the relative atomic weight of twentyone bodies, gaseous, fluid, and solid; - and that in May, 1808, he published his 'New System of Chemical Philosophy,' embodying these various results of the continuous labour of years. In a letter to his brother Jonathan (March, 1803) 
he describes himself as having ' got into a track that has not been much trod in before.' This was the track, and these the discoveries to which it led him.

Pursuing farther onwards the history of their progress, it must be stated that Dalton's views, even when already matured by proof, did not find instant adoption in the scientific world. We have already alluded to his uncouth and ineffective manner of propounding them, whether by lectures or writing. The very phraseology of atoms and atomic weights frightened timid reasoners away from the subject; and the boldness of the diagrams by which he depictured his groups of spherical atoms strengthened the belief that it was a rash recurrence to the tenets of an exploded philosophy.* But the husk, however thick it be, is always pierced through when truth lies within the kernel. A few eminent chemists, among whom Thomson, Wollaston, Gay-Lussac, and Berzelius may especially be named, speedily saw the value of the discovery, and applied their own labours to verify and extend it. Dr. Thomson's habitual zeal was quickened by personal communication and friendship with Dalton. Of Dr. Wollaston it was said that he would soon have made the discovery himself if Dalton had not done so; and the mathematical acuteness of his understanding, as well as the direction and method of his chemical researches, might justify this belief, were it not that a certain scepticism of mind perpetually checked and impeded the efforts of which his intellect was capable. His paper on super-acid and sub-acid salts furnished much collateral testimony to the truth of the doctrine; while

* It was Dalton's opinion that no conception of this kind could be clearly grasped by the understanding, without some embodiment to the sight. The best sanction to the justice of his views is the adoption of this manner of illustration by one of the most eminent chemists of our day, Dr. Hofmann; who, in his admirable lectures on Organic Chemistry at the Royal Institution, denoted the most complex organic compounds by coloured atomic symbols; so disposed on sliding frames that every change, whether by addition or subtraction of atoms, could be shown with the utmost ficility.

E E 
his sliding-scale of chemical equivalents offered a very ingenious instrument for its application to practical chemistry. In determining by beautiful experiments the law of combination of volumes in equal or multiple proportions, Gay-Lussac added fresh evidence, better appreciated by others than by Dalton himself. Meanwhile Berzelius, in his Northern laboratory, executed those numerous and admirable analyses, which, fulfilling in their results every condition of the atomic theory, obtained for it the general acquiescence of the scientific world. The tables he constructed of atomic weights have required little change but that of enlargement. The ingenious system of chemical formulæ which he devised, denoting these atomic relations, was speedily accepted by chemists and has since been very generally employed.

The remaining history of the progress of this discovery as it comes down to our own time, and of its influence in every branch of physical research, cannot so easily be made clear to our general readers. The chemists of the present day more especially, deriving impulse from the atomic doctrines, have carried these as instruments into parts of science hitherto inaccessible to research; fully attesting by their success the validity of the means so employed. The whole domain of Organic Chemistry may be said to be a recent conquest thus obtained. The laws of isomorphism, of isomerism, of atomic substitution, of compound radicals, have all been determined during the same period by the genius and labours of Mitscherlich, Liebig, Hofmann, Dumas, and other foreign and British chemists. It would be beside our present object, and indeed impossible here, to give any adequate idea of these abstruse and difficult researches. We allude to them only to mention how completely they are interwoven with the atomic principle, and how thoroughly they illustrate its various workings in the natural world.

There are one or two points, however, of which we may 
speak somewhat more in detail, as best illustrating the nature and value of the objects attained, and the method of their attainments. Two great steps of progress are especially to be noted in the discovery. The first in order of time as well as in order of thought and deduction, is the Law of multiple proportions; originally derived, as we have seen, from a certain class of compounds - the neutral salts - and afterwards extended so largely to others as to assume the character of a general law of chemical combination. The principle briefly stated is, that when bodies, whatever their form, enter into. such combination or separate from it, they do so in the same fixed proportions; - that these proportions are either equal or multiples of one another; - and that intermediate quantities or proportions are never found to exist. The earlier exceptions to this law have been gradually removed as chemical analysis acquired greater exactness; a striking evidence in itself of the truth and completeness of the discovery. Cogent, however, as were the facts upon which it rested, there was at first some hesitation among chemists in adopting it. The eminent name of Berthollet had just given currency to the doctrine that the mere quantity of a combining ingredient, irrespectively of other conditions, was largely concerued in all chemical affinities and combinations; and this opinion was ably defended by other chemical writers. The controversy, however, could not long be maintained in the face of evidence almost mathematical in kind, and all other views speedily merged in the one doctrine we have just stated.

Here, then, was a mighty gain effected not merely to Chemistry, but to physical science in its every part. By introducing the function of numbers in scrutinising the wonderful changes which take place, invisibly to us, among the molecules of bodies in chemical action, we may be said to have gained nearer access to one of the great facts of creative intelligence. For if these elementary molecules are 
thus governed by numerical relations in every act of union or separation, it is obvious there must have been some primary conformation or adaptation which alone could fit them to fulfill this condition. And this conclusion is alike valid, whether we adopt the number of bodies which are regarded as elementary under our present knowledge, or admit the more probable belief that this number will be greatly reduced hereafter by new methods of analysis.

We have mentioned the neutral salts as first yielding the principle of multiple combining proportions. The combination and separation of gases by volumes afforded the same result, and by an inference still more simple and direct. Two gases, chemically uniting to form a compound, invariably combine in the same measured volumes of each, or in multiples of one or the other, if there be more than one product of this union. The same precise proportions are found in the separation of the compounds thus formed. There is a peculiar grandeur to those who look on nature aright, in the simplest forms of demonstration of a great natural law. We feel this when having before us the two glass tubes, receiving severally the streams of oxygen and hydrogen which arise from the decomposition of water - one volume by measure of the former to two of the latter - and these proportions so absolutely exact, that the nicest graduation of the tubes can detect no deviation in the results. These results represent to us not merely the contents of two small tubes, but the relative volumes of the same elements contained in, and forming by their union, the total mass of waters on the globe.

Out of this great law of multiple proportions in all chemical compounds arose the further discovery, more especially due to Dalton, of the relative weight of the combining molecules or atoms - another vast step in the progress of natural science. The method of the discovery is as striking from its simplicity as the result from its grandeur. The reasoning of 
Dalton ran thus. If in the chemical union of any two simple bodies we suppose a single atom of each to form the combination, then if by analysis we obtain the precise relative weights of the two in the compound, we gain in effect the relative weight of the atoms thus combined. Take the case of water in illustration. The uniform and peculiar characters of this wonderful fluid suggested the inference which Dalton adopted, that it is formed by the union of a single particle of hydrogen to one of oxygen. Analysis proving the relative weight of the two elements in water to be as 1 to 8 , the conclusion was immediate, that the same ratio represented the weight of the atoms of hydrogen and oxygen respectively in this fluid.

But more was needed yet to justify this conclusion, and some third body required to be brought into the chain of evidence. Taking carbon as the instance, and examining carbonic oxide gas, - the simplest form of its combination with oxygen, - we find the relative weights of the two elements in this compound to be 6 of carbon to 8 of oxygen; whence it is inferred that an atom of each enters into the compound, and that the relative weights of these atoms are as the numbers given. Thus, then, we have obtained three proportions of weight: 1 for hydrogen, 6 for carbon, and 8 for oxygen. Now mark the beauty of the evidence. If these three numbers be correct, we ought to find them recurring, either in simple or multiple form, in all the compounds of carbon and hydrogen, thus completing the circle of proof. And such is actually the case. The details of this demonstration belong to Chemistry; but the more they are extended to different substances, simple or compound, the more complete and striking does the evidence become.

Stopping a moment here, let us see precisely how we are to define the knowledge thus far gained. One essential point will already be understood, viz. that in speaking of atomic 
weights, it is only the relative weight of the atoms of different substances, which enters into the question. The term Atom denotes the simplest and smallest combining proportion of any body. Of their positive weight in relation to any known quantities we are absolutely ignorant; nor has any path been disclosed through which such knowledge may hereafter be reached. We can hardly say that the value of the discovery is less from this abatement of its extent, since for all purposes of science the relative weights are always the object sought for, and carried into use.-Again, these discoveries teach nothing certain as to the figure or absolute magnitude of such primitive atoms. Dalton, indeed, was led by various considerations to regard them as spherical, and to delineate them as such. But this, however probable, is still speculative to our knowledge; and certain isomorphic phenomena might rather suggest several primitive forms than that of the spherical atom alone. Such inference is especially justified as regards the combining atoms of compound bodies. Here theory, rational in itself, somewhat outruns actual knowledge; but is continually and closely followed by it.

As respects the question of magnitude, all that can be sought for with any hope is the relative magnitude of these elementary parts. This enquiry, though far less simple and certain than that of relative weights, has been pursued with much zeal and some success both by English and German chemists, though under the form of comparison of atomic volumes rather than atomic diameters. The distinction here involved, and the general relation of volumes to other conditions of matter under the atomic theory, would have demanded further notice had the limits and design of this article made it possible. We may briefly say, that the estimate by volumes, though never willingly acquiesced in by Dalton himself, fully confirms in every part the principles of the atomic theory; and as respects its particular application to the discovery of 
relative magnitudes, though the results are still neither certain nor complete, yet the numerical relations and analogies they already afford are very remarkable, and will probably conduct to new and higher proofs of the doctrine before us. In ascertaining the nearly identical atomic volume of certain groups of elementary bodies, closely allied in chemical properties and found generally in close association in nature, MM. Kopp and Schröder, to whom chiefly we owe these researches, are evidently on a path which promises access to yet untrodden fields of science.

We shall advert but to two or three of the many general relations which subsist between the Atomic theory and the various branches of physical science. The first of them is expressed in the phenomena of Crystallisation - those mutual actions of the molecules of matter, whether simple or compound, denoted by the assumption of regular or geometric forms. Crystallisation may almost be defined as a conversion of atomic numbers into a material configuration. In all the vast variety and beauty of crystals, giving splendour to our mineral cabinets, and illustrated by the labours of Hauy, Mohr and Weiss, Wollaston, Brewster, Beudant, \&c., we find proof of the primitive tendency of atoms to group themiselves in certain definite figures; - to build up, as it were, the crystalline fabric in mathematical lines and proportions. When to this we add the fact, that changes may take place in the solid interior of a crystal without affecting its outer form, we gain some idea of those wonderful workings of molecules or atoms, which in their totality make up all the phenomena of the material world.

Connected with this topic, and perhaps but a more general expression of the same physical law, comes in that great principle of Polarity; - hard to define in its most extensive sense, and yet so largely seen and proved in every part of nature, that no science can be successfully pursued without 
a constant regard to it. To the Atomic doctrine especially it is essential, almost as an integral part of the theory and as the sole exponent of the phenomena. We apply the term polarity to various actions of light, magnetism, and electricity, but in reality we know such actions only by the polarities given to the particles of matter upon which these forces act. The whole subject is one of deep interest to the futurity of physical science, and its connection with mathematical as well as experimental enquiry is the best security for the truth of every result obtained.

It is strange, yet nevertheless in accordance with the light and shade of all human things, that while true science is thus embracing in its progress every part of the material world - establishing facts, disclosing laws, and scrutinising by assured methods even those actions and relations of matter which are inapproachable by the senses - there should exist simultaneously a series of delusions, in which credulity and imposture are closely interwaven; yet usurping the phraseology of real science, and thus misleading many whose intellect in other matters is acute and discriminative. We would fain indulge the hope, recently expressed by one of our most eminent philosophers, that education may in the end provide a remedy against these aberrations; but we still fear that as long as credulity remains a part of the human temperament, there will never be wanting the agents and materials to work upon it. Our best guarantee lies in the knowledge that follies and impostures are in their nature ephemeral; while no fact of genuine science can ever disappear, except by merging in some higher and more general truth. 


\section{MODERN CHEMISTRY.*}

[Quarterly Review, No. 165.]

T $\mathrm{N}$ giving the titles of these systematic works on Chemistry, 1 to which many others might be added, we must not be understood to intend an analysis of their contents, or even a critical comparison of their merits. Chemical science has become far too vast and complex a subject to be dealt with by any summary in the pages of a Review. It stands apart from and beyond the margin of critical literature. Yet, as we have been accustomed, from time to time, to place before our readers those works which more strikingly illustrate the progress and revolutions of physical knowledge, we would now use the volumes before us as the foundation of a brief sketch of some of the great changes which Chemistry has undergone within the last fifty or sixty years, and notably within the latter half of this period. A general statement of the principles and present methods of the science, and of the more remarkable discoveries to which they have led, is all we can undertake to give; with no other details than such as are needful for illustration.

Even this limited outline is not without its difficulties, seeing the magnitude and variety of the changes that have

* 1. Elements of Chemistry. By the late Edward Turner, M.D., F.R.S. Eighth Edition. Edited by Baron Liebig and Professor Gregory. London, 1847.

2. Elements of Chemistry. By Thomas Graham, F.R.S.L. and E. Second Edition. Part I. London, 1847.

3. Elenents of Chemistry, theoretical and practical. By William Allen Miller, M.D., V.P.R.S., \&c. Second Edition. 
occurred; and their intimate relation to the state and progress collaterally of the other physical sciences. They are revolutions depending not solely on the accession of new facts, but involving also new principles and methods of research ; - a larger scope and more profound objects of enquiry, and modes of experiment infinitely more subtle and exact wherewith to attain them; - and with all this, an altered nomenclature and new symbolical language, needful to meet the exigencies thus created. A chemist of forty years ago, well versed in the subject as it then stood, would be utterly lost in the labyrinth of new names, new facts, and new combinations which appear in the works before us. This is true, even as to the elementary parts of the subject, and what is called Inorganic Chemistry:- yet more true as regards the wide domain of Organic Chemistry, a land newly opened, rich in products, and cultivated with such zeal and-success, that any one stationary in knowledge, even for half the time we have named, would enter it as a stranger to all he saw around. We might give pâssages without number, taken almost at random from the descriptive parts of organic chemistry, which would come upon the eye of a reader of the old chemical school with the same obscurity as a page of 'Saunders on Uses' or 'Sugden on Powers' on the mind of the youthful student of law first opening these mystical volumes.*

In attestation of the same fact we find that the chemical writings of greatest reputation thirty years ago - the original works of Fourcroy, Berthollet, Thomson, Murray, Henry, \&c. - are now utterly out of date and useless. Even those which replace them to the modern student have their value

* Pentethyline-tetrathylated-tetrammonium;-Dibromide of Ethylene-diethyldiammonium, are names before me at this moment in a recent paper by Dr. Hofmann presented to the Royal Society.

It will be seen that each of these names is in effect an analytical description of the compound body under observation. 
mainly determined by the lateness of the edition; and follow with difficulty the rapid and incessant progress of research, and the changes of doctrine, as well as facts, which they have to record.

The present condition of Chemistry wears a still more marvellous aspect, if we regard it in relation to all ancient knowledge on the subject. The physical philosophers of antiquity hardly reached its borders, and never fairly crossed the threshold of the science, or recognised the great principle of enquiry which it.involves. Experiment in their hands was accidental and insulated, seldom adopted as a deliberate means of extending knowledge or attaining truth. However it be explained, the methods of the Greek philosophers, and the mental temperament of this people, would seem to have withdrawn them from this only path to truth. And if they failed in apprehending the principle of experiment, as applied to the objects which form the science of Chemistry, we have little reason to look for such discovery among the Romans, or during the ages following the disruption of their empire. We cannot attach the value some have done to the studies of the Arabian chemists, or the partial and ill-directed pursuits of the alchemists; who, though bequeathing a certain number of terms to us, can scarcely be said to have used experiment as a deliberate principle of research, and left little that has been finally incorporated into the chemistry of the present age. Had our countryman Roger Bacon lived at a more propitious period, seeing his spirit and methods of enquiry, we may believe that he would have held high rank among the discoverers in the science. It would be idle to repeat what has been so often said of his great successor in the lapse of time, Lord Bacon - the first who fully indicated experiment and exact observation as the only road to physical truth, and gave a definite classification of its objects, tending to the right order and direction of pursuit. It is an error, 
however, to imagine that the path disclosed by this extraordinary man was at once recognised and followed by others. As respects Chemistry in particular, we find that it assumed its true and complete character as an experimental science, at a later period, and from causes which would have existed had Bacon's writings never appeared. We refer to them rather as a striking anticipation of the methods of experimental enquiry, than as having given origin to those results of modern discovery which are now daily multiplying around us.

This slight allusion to what has been called the early history of Chemistry will show that we do not attach other value to it than as a record of the insulated progress of man in various arts, useful or useless, which have chemical processes for their basis. The workers in metals from TubalCain downwards, and those engaged in the cookery of human food in every age and country, have dealt with chemical powers and instruments, though not recognised as such. But it would be as reasonable to call the Bee a philosophical chemist, as so to designate those who have simply invented means to satisfy the needs, or minister to the luxury of man. Chemistry illustrates these familiar phenomena, but does not depend on them. Nor can it be affirmed that any of the greater discoveries which mark its progress have had their origin in the wants of human society, though often directed and pressed forwards by this powerful incentive.

Without affecting exactitude, there can be little error in stating that since a century only Chemistry has assumed its true character as a science, and that, with few exceptions, all the important parts of its early history are included within this period. The eminent names of Boyle, Hooke, Mayow, Stahl, and Hales do indeed precede it; but not until the period between 1750 and 1790 (a time illustrated by the greater names of Black, Cavendish, Watt, Priestley, Bergman, Scheele, and Lavoisier; and by the discoveries of oxygen, 
hydrogen, nitrogen, and carbonic-acid gas; of latent heat, elective affinity, the composition of water and atmospheric air, and the true nature of oxides and acids) can we consider Chemistry to have acquired the foundation upon which it now rests. Allied to the other experimental sciences by similar methods of enquiry, yet vaster and more various in its objects, it has undergone greater changes and expansion than any besides; absorbing into itself some of these collateral branches, and assuming such relation to others as to indicate a future time when they may all be comprehended under some more general system of physical truths.

We have already named it as our object to present a short outline of the more important changes and steps of progress which mark the recent history of Chemistry; not limiting ourselves to chronological order, but seeking what may best illustrate the principles and present doctrines of the science, and those methods of research by which it has attained its actual condition. In doing this, we shall avoid as far as possible all technicalities of language, and take such illustrations only as may be easily understood. A few general remarks, however, are necessary in preface to those more particular points on which we shall afterwards speak.

In a recent article of this Review, we mentioned what we consider the two most striking characteristics of modern physical science, viz. the more profound nature of the objects and relations with which it now familiarly deals, and the wider generalisations thence obtained; and 2 ndly, the greater refinement and exactness, both of observation and experiment, with which these objects are practically pursued. Chemistry affords some of the most remarkable instances we could select; and the more closely we examine its present state, and growing connection with other branches of physical science, the more striking will these illustrations appear. 
In truth, it can hardly be defined or described so as not to include its great purposes and powers; and, together with these, that exactness of methods upon which its progress mainly depends.

All great truths, whether of morals or physics, are marked by their simplicity. Although not an absolute test, since false principles or paradox often seek shelter under the same forms, yet we may affirm that in proportion as truths become more complete and comprehensive, so are the expressions appropriate to them more simple and determinate. And this is especially the case in regard to physical knowledge. Though facts have multiplied, so as to encumber the mind of the student, and seemingly to dissever the material world into endless fractional parts, yet has the discovery of new relations and connections tended unceasingly to reduce these facts under more general laws, and to give to science a simplicity of higher kind at every great step in its progress. To what future point this process of integration may proceed, we hardly venture to surmise. We may, however, express our belief that we stand but on the margin of what will be hereafter attained.

We have already said that Chemistry, from its nature and objects, forms of necessity a principal basis of such progress. And this brings us at once to the definition of these objects - a definition which, simple as we may seek to render it, must yet be made to include actions infinite in number and variety - different throughout all forms of matter - ministering directly or indirectly to all the phenomena of the natural world - and essential to the being and maintenance of every form of animal and vegetable life. Chemistry can only be described by a generalisation which will embrace all these conditions within itself.

Matter is presented to us in the universe at large, as masses acting upon each other in obedience to the law of 
gravitation, while on the globe we inhabit it appears under innumerable forms - simple or compound - solid, liquid, or gaseous - all subject to the same great law, and also to the various physical conditions of cohesion and repulsion, acting on like particles, and producing many of what are called its secondary properties. But beyond these conditions, and distinct from them to our present knowledge, comes in that mighty force which we term Chemical Affinity - the power of attraction at insensible distances, uniting dissimilar particles of matter, and by its various intensity and manner of action, producing the vast assemblage of material phenomena which we now class under a common name. Chemistry is the science which investigates this affinity, positive or relative, of the molecules of material substances - the laws which regulate their combination and separation - and the results of the actions and changes thus produced. It takes cognizance of the great elementary forces of heat, light, and electricity, in their ever constant influence on all such actions and affinities; and it may hereafter, as we have said, lead us to physical relations and laws of a higher class, in which these elements and the integral properties of matter are still more closely blended. The science, however, is essentially one of experiment; and its eminent progress of late years is mainly due to the clearer definition of its objects, and to the enlarged methods and improved instruments with which these are pursued.

In describing it as the office of Chemistry to determine the mutual affinities of the atoms of bodies, and the results thence derived, we use the term in a sense justified by our actual knowledge. The history of the Atomic theory, as originally derived from the barren speculations of ancient philosophy, and in our own time appropriated to the highest purposes of true science, has been fully given in a preceding article. Our business will be now to indicate the effect which this doctrine, so applied, has had upon the whole school of 
chemical philosophy, and especially on Organic Chemistry as one of its great departments.

The procedure of Chemistry, as an experimental science, may be resolved exclusively into the two great methods of Analysis and Synthesis; - the separation of parts before united, or the union of parts before separate. No chemical operation can occur in which one or other of these changes is not concerned; and the attainments of the science are best estimated by the facility and exactness with which such changes are effected. Of these two methods, both depending on the relative affinities of different kinds of matter, analysis has a natural precedence. Even in the processes of nature the separation of compounds is more obvious than the reunion of parts. The changes and combinations upon which organic existence depends - forming the chemistry of animal and vegetable life - are slow and occult processes compared with those which dissever such combinations, and restore the parts to more elementary state. And when the subject assumed the character of an experimental science, the chemist found himself surrounded by innumerable compound bodies, readily decomposed, and suggesting that more formal analysis which might collect the parts, determine their nature, and fix the proportions in which they severally exist.

The method of synthesis comes in natural sequence to this; affording a test of the truth of analytical results, and satisfying a rational curiosity as to the effects of new combinations among the innumerable forms of matter around us. In both these operations however, and as a first principle of all chemistry, it is to be kept in mind that no matter is either created or lost, whatever the changes or combinations taking place.* In clearly fixing this principle, which was

* Plutarch ascribes to Empedocles a passage which is well descriptire of this great principle of Chemistry:-

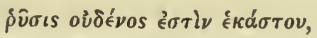

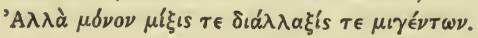


imperfectly apprehended before, Lavoisier rendered a service to science, better deserving of record than many discoveries which have higher fame with the world. It is an axiom of which every single chemical phenomenon testifies the truth.

It may seem that we are dwelling too long on these elementary points; but in seeking to give a summary view of the changes in modern chemistry, such preliminaries are essential to a comprehension of the subject. The changes in question include, not discoveries of fact only, but alterations and extensions of the methods of enquiry; sufficient to give a new aspect to the science, even apart from the great results to which they have led. This invention of new means and instruments of research is, in truth, a topic of the deepest interest. The sudden enlargement of power thus obtained, and the faculty of penetrating into parts of nature before hidden or obscure, place such discoveries among the highest class of human attainments, and render them epochs in the history of human knowledge. In Chemistry especially, the reciprocal dependence and felicitous union of new facts and new methods may be said to give a geometrical power to the science; as in that eminent discovery which taught the universality of definite proportions in chemical combination; and by establishing this fact, placed instruments in our hands, wholly new in their manner of operation, and far more powerful than any before possessed. Reverting to this afterwards, we may notice meanwhile some of these methods, which though not actually new, yet so greatly transeend all anterior use, that they virtually become so, and may rank among the recent revolutions of the science.

The first, already alluded to, is the wonderful increase of exactness in every part of chemical enquiry. Those only who are conversant with its history can form an adequate idea of the amount of this change, or of the influence it has had 
on the progress of the science. Pervading every part of the subject, from the simple observation of external physical appearances, to the most complex and subtle forms of experiment in the analysis and synthesis of organic bodies, its value is more especially felt in these later and higher operations. The perfection of analysis, in its conjoint relation to qualities and quantities, is in truth the cardinal point of all chemistry. We might give curious contrasted examples of the grossness of this operation fifty years ago, and the exactness it has attained at the present day. Whether it were the examination of a mineral water, or a metallic ore, or an animal or vegetable product, the older analysis seldom yielded half the ingredients which are now derived by the chemist from the same material of experiment. The greater number lay hidden under the imperfection of the means used to separate them. It is true that those indicated were generally the most important, and present in largest quantity. But it often happened that the ingredients, thus latent under ruder forms of analysis, were really essential elements in the compound; modifying its physical qualities, determining its relation to other chemical agents, or providing for its uses in the economy of nature. Thus what was recorded as loss or undefined result - the mere residual dross of ancient analysis - has become richly prolific to modern research; affording those rarer products, which, while they seem to encumber our chemical tables by their number and diversity, do in effect present so many fresh objects of enquiry, and give promise of the disclosure of relations hitherto unknown.

To refer to particular instances of this great change might detract from what we wish to convey of its universality. A single illustration may be taken from the history of Iodine and Bromine - substances discovered some forty years ago in the waters of the sea, and in certain sea plants - remarkable as new and elementary forms of matter to our present know- 
ledge - and possessing properties so peculiar, as to make it certain that they fulfill definite, though unknown, purposes in the economy of the globe. The exceedingly minute proportion in which they are found in nowise disproves this. Chemical energy is only partially dependent on quantity; but were the latter alone concerned, it would be enough to estimate the enormous mass of ocean waters of which they form a part, to convince us that they cannot be inert or indeterminate in the objects of creation.* To the new substances, so discovered, the refinements of modern chemistry have been incessantly directed. By delicate and beautiful experiments they have been detected in numerous mineral waters and brine springs, and even in certain metallic ores. They have been brought into close relation of analogy with other great chemical agents, as oxygen and chlorine, and into artificial combinations of endless variety; - and these combinations have already yielded new remedies to the physician, new agents in experimental research, and the most refined methods wherewith to determine the chemical actions of light, and to give them their happiest application in photography. In the progress of these researches tests have been attained so delicate, that iodine may be detected in a liquid containing much less than its millionth part by weight; the familiar substance of starch affording this subtle test, by its chemical relation to the element in question.

Without protracting this illustration by further details, we may briefly state that the same exactness and completeness have been carried into every part of Chemistry. Chance, vague hypothesis, and crude results, are altogether excluded from the science. Weight and proportions, numerically

* In a former article mention has been made of the discovery of Lithium in sea-water; but in quantity still more minute than that of the two elements spoken of above, and discoverable indeed only through the refinements of the spectrum analysis. Yet, if found in all sea-water, and in the same proportion, its presence cannot be a casualty or without some purpose fulfilled. 
expressed, form the basis and test of experiment; and exact cognizance must be had of every quantity gained or lost by the substance operated upon. No conclusions are deemed perfectly valid unless so substantiated. This higher principle of research - mainly due in its origin to Lavoisier - has given such perfection to chemical theory as applied to analysis, that the chemist can often foretell results before entering his laboratory; and experiment comes rather as fixing and completing the deductions from general laws than as disclosing facts previously unknown. In our own country Dr. Wollaston contributed perhaps more than any other to the exactness of experimental enquiry. If we name Berzelius, Mitscherlich, Liebig, and Dumas at the same time, we do so with hesitation, lest we should seem to disparage other great Continental chemists whose labours have tended to this perfection of the science they profess.

While referring to the increased exactness of all chemical knowledge, we must give the-statement a more particular application to whatever concerns the influence of small quantities in composition. We have already adverted to the frequent case of an ingredient existing in very minute proportion, yet conveying important or even essential chemical properties to the compound of which it forms a part; - or what is an analogous case, to the effect of a slight change in the proportions of one ingredient in altering the qualities of the whole. Modern chemistry is replete with instances of such facts; the proper estimate of which is indispensable to the understanding of the science. It has belonged to the refinements of recent analysis to detect, and assign their proper value to, these more minute ingredients; - while at the same time discovering many new and rare elements, and indicating purposes which they fulfill in the economy of nature, even by virtue of their diffusion and minuteness. In organic chemistry, whether of animal or vegetable life, we find this admirably 
exemplified; and to the consummate skill with which such small quantities have been estimated, we owe some of the most striking discoveries in physiology, agriculture, and the arts of life.

We have cause indeed to presume, that whenever a particular element is generally present in a compound, and in definite proportion to the other ingredients, such element is essential to its nature, however small the proportion may be. This principle has been constantly confirmed as chemical knowledge advanced; and it becomes now the expression of phenomena, which may well astonish those not familiar with the subject. It is exemplified by the carbonic acid present in the atmosphere, in a proportion not exceeding $\frac{1}{1000}$ th part of its weight; - and presumably also by the iodine, bromine, and lithium, in the waters of the sea, though here, as we have seen, the proportion is yet infinitely smaller. The iron existing in a portion of the blood - the phosphorus found in the medullary substance of the brain and nerves - the fluoric acid in bones - the sulphur in albumen, fibrin, and certain other animal matters - and the silica, sulphur, phosphorus, and various metallic oxides or alkalies, found in different vegetable substances - are a few among the many examples which organic chemistry furnishes of the influence of minute quantities in composition. They are relations of deep interest to us, as wonderful and exquisite provisions of Providence for the purposes of life, and for the mutual dependence of the several parts of creation. What they present in natural combinations has its counterpart in the artificial chemical union of different substances, where we still find under various forms this marvellous influence of small quantities, pervading and changing the sensible properties of large masses or volumes of matter. We can destroy the ductility of gold by exposing it, when melted, to the mere fumes of antimony. We can variously change the physical properties of other metals by 
an amount of alloy much less than a thousandth part their own weight. And there are cases where a proportion of calcareous matter, much smaller still, suffices to alter the sensible properties of the substances through which it is diffused.

So numerous, however, are the instances of this nature in Chemistry, that the citation of a few rather impairs than enlarges the conception of the great principle they involve. They form, in truth, part of that great attainment of modern science, the discovery of conditions and laws of molecular change in the interior of bodies - of those subtle interpenetrations of matter under the influence of light, heat, electricity, or chemical force, and often independently of the outer forms or densities of the bodies acted upon - the study of which brings us among the more occult relations of the material world, and promises access to physical truths yet higher than any hitherto attained.

In estimating the influence of these small quantities in composition, some chemists, and particularly Liebig and Dumas, have pursued a method, partially adopted before but in a manner far below the perfection of present use. This consists in calculating, through the proportion of parts, the absolute or approximate quantities of matters thus minutely diffused;- expressing them in weights or volumes; - and thence deriving results inaccessible by other modes of research. The positive amount of carbon present in the atmosphere, for example, is a question of much interest to the theory of vegetation, and to other phenomena of the earth's surface. This question is solved, first, by estimating - (which can be done exactly) - the total weight of the atmosphere surrounding the globe; - next, by taking the fractional proportion which carbonic acid forms of this amount; - and, finally, by deducting the further proportion which oxygen bears in the composition of the carbonic acid; giving as a gross result 3,085 billions of pounds of the element of carbon existing in 
the atmosphere; - a quantity which Liebig states, but on less assured grounds, to exceed the weight of all the plants and strata of coal existing on the earth. The same method has been largely and curiously applied to the ingredients of animal and vegetable bodies, and to the parts of inorganic nature on which they respectively depend; with results very interesting in the natural relations thus disclosed, and of great practical utility to agriculture and other arts of life.

A further circumstance, characteristic of modern Chemistry, is the great extension of what may fitly be called the creative part of the science, forming one of the most eminent attainments of physical enquiry. The refinements of analysis, already noticed, are even less remarkable as proofs of advancing knowledge, than are the multitudinous combinations which the chemist obtains from the materials submitted to his hands. Creations, in one sense, we may venture to call them; since a large proportion of the compounds thus artificially formed have no actual prototypes, as far as we know, in the world that surrounds us. They do not exist elsewhere than in the laboratory or manufacture, where a happy accident or happier skill has produced them. That supreme dispensation of the Almighty, of which the term Nature serves but as an humble exponent, has placed us amidst matter in different forms - lifeless and inorganic, or organised into life,- but equally committed to us to mould into new combinations, serving to our uses or satisfying our curiosity. Human invention, accident, or necessity have from the earliest time created these combinations. They become of far greater complexity and more refined use, as science and civilisation proceed in their course. The chemist of our own day, though not without practical motives at a time when all worldly interests are in a state of such intense activity, has carried the labours and results of pure science 
far beyond any calculation of this nature. Commanding new resources of experiment, and possessed of the true laws of chemical combination, he pursues the various forms of matter, whether simple or compound, throughout all their relations and affinities; - obtaining in his progress, and as a result of these affinities, numerous substances wholly unknown before, yet possessing qualities as singular and strongly marked as those which Nature herself proffers to our enquiry.

Examples of these remarkable products of synthetical chemistry might be endlessly multiplied. In mentioning iodine and bromine we noticed the numerous and complex combinations they have been made to assume; - all of great interest from the relation of these two bodies to other undecomposed elements around us. When speaking of Organic Chemistry, we shall have to notice the production artificially of certain organic compounds, not to be distinguished from their prototypes in nature, and forming in this respect a discovery which may well rank among the most eminent in physical science. Of other instances we shall take only a few, for mere illustration. Every new metal discovered(and the activity of modern research has more than trebled the number known to antiquity) - has been followed through a long series of combinations with other chemical elements, all determined by the law of definite proportions; yet, while obedient to this great common law, yielding numerous products altogether new to us and to the natural world. Some of these are of eminent utility to man; others possess properties of strange and fearful kind; - such as those explosive metallic and gaseous compounds, of which the parts seem to be compelled into an unstable union, prone at any instant to sudden and violent dissolution. Gunpowderthat extraordinary substitute of chemical force for manual or mechanical means of destruction - cannot historically be called an invention of Chemistry, though expressing curious 
and complex chemical actions. But the explosive-cotton, recently discovered, is peculiarly the product of chemical research; depending on very singular affinities, which have already been variously applied.

Another striking example of this chemical creation is the Protoxide of Nitrogen - the intoxicating gas - a particular combination, in slightly different proportions, of the oxygen and nitrogen composing the air we breathe; but nowhere existing in nature under the form in which science presents it to us. The admission that atmospheric air is a simple intermixture of gases, and not a chemical compound, scarcely abates the wonder that so small a change in the proportions which minister to common life should become the cause of those sudden and strange affections of the brain and nervous system, which alter for a time the whole condition of the being. Chemistry, however, and especially organic chemistry, accustoms us to these wonders. Equally striking in their properties are the two creations of the laboratory, Sulphuric Ether and Chloroform. By working with and among the affinities of certain elements, man has obtained these compounds - (and there are others of kindred quality) - the simple inhalation of which produces insensibility to pain, even under operations the most severe which surgery can inflict. We have spoken much of chemical analysis. This is in effect an analysis of the compound nature of Man; the separation and removal for a time of a part of our sensitive existence; - having close analogy indeed to certain of the conditions of sleep (itself the great mystery of life), but even more striking in some of the inferences it conveys; and, unless it be that bodily suffering is allotted to us for moral uses, a discovery profuse of benefit to the human race.

It would be easy to multiply similar examples of the generation of new compounds, remarkable in physical properties or in their physiological effects. We will give but one 
instance more, and this rather from its whimsical nature, than from any connection the substance is likely to have with the uses or ornaments of life. Discovered a few years ago, as a definite compound of arsenic, carbon, and hydrogen, it has been called Kakodyle from its peculiar smell - an odour so intolerable, that even the chemist, inured as he is to vile and noxious exhalations, seems to have shrunk back for a time from the work of his own hands. Yet such was the interest attached to this substance, as a compound organic radical, fulfilling in composition the part of an elementary body, that despite the quality just named, and the virulently poisonous and inflammatory nature of its compounds, it has been followed through all its combinations with consummate care; and no disgust or danger have checked a single experiment which could illustrate the complex affinities of which it forms a remarkable example.

We have thus far been speaking of the general principles of Chemistry, and of those neî methods which have so largely extended its dominion over the material world. In doing this, we have sought illustration from some of the many discoveries crowding the later periods of the science; to the most important of which we may now more explicitly allude, in completion of the view of the actual state of Chemistry which we are seeking to convey.

We have already noticed that remarkable series of discoveries illustrating the latter half of the 18th century; the separation and definition of the gaseous bodies - the decomposition of water and atmospheric air - the doctrines of latent and specific heat - the determination of the true nature of oxides - the principle of elective affinity, \&c. To these points, now so familiar, we shall not further advert than by repeating that they gave foundation to Chemistry as a science, and furnished instruments and guidance for the 
rapid progress it has since made. The first ten years of the present century were marked by several great discoveries, and by that one, signally pre-eminent above the rest, of which we have largely spoken in a preceding article.* We allude to what we have there called the Atomic theory; but which for our present purpose may better be termed the doctrine of definite and equivalent proportions in chemical combination. Some idea of the importance of the principle may be gained from this mere enunciation; but its real extent and value, and the place it holds among the great laws of the material world, can only be understood by those well instructed in physical knowledge. Of the history of the discovery in its several stages, we have sufficiently spoken in the article referred to. Our object is now to denote some of those remarkable results which have been derived from this discovery in its application to every part of chemical research and philosophy.

Chemistry we have before defined as the science which regards those mutual attractions or affinities of the elementary molecules of matter, upon which all its laws and phenomena depend; - varying for each particular kind of matter, simple or compound; and varying for each in relation to matter of every other kind.

It was speedily seen that the term Atom, in denoting the simplest and smallest combining proportion of any body, became, though not a necessary, yet a justifiable exponent of the great physical principle thus developed; and one in truth which almost ceased to be hypothetical- when experimentally applied. Scientific theories required that our conception' of the divisibility of matter should approach the borders of the infinite, but rest nevertheless within the finite; and all scientific research corroborates this view. The actual

* See the preceding article in this volume, on the 'Life of Dalton, and the Atomic Theory.' 
minuteness of the molecules of matter, as rendered certain by experiment, can be expressed to the understanding by numbers only. We have mentioned some instances already; such as the detection of a millionth part of Iodine in solution. In Faraday's experiments on the optical properties of Gold solutions, he found that a strong ruby tint was given to a fluid by a proportion of gold not exceeding the half-millionth part in weight. Examples of the same kind are endless, and furnished by every part of nature, organic and inorganic. But none approach in wonder those recently derived from the spectrum analysis. By experiments conclusive in their method and results, Bunsen was able to detect $\frac{1}{70,000,000}$ th part of a grain of Lithium in a compound; while of Sodium, the metal of common salt, the delicacy of the indication by the yellow line traversing the spectrum, was such that the $\frac{1}{180,000,000}$ th part of a grain could be made clearly perceptible in its effects.

We must not stop here to relate the ingenious methods through which these conclusions were obtained, or to give other examples to the same effect. They all show that in the primitive constitution and multiform changes of material bodies, nature works with molecules or ultimate parts of indescribable minuteness; which minuteness we may conclude from the phenomena to be absolutely essential to the functions they fulfill. Equally essential does it appear to be, that these molecules should tend to unite under certain definite forms; and, where dissimilar in kind, under certain fixed proportions of number or weight. In this law, if we may so term it, lies the explanation, both of the uniformity and of the diversities pervading every part of the natural world. The whole system of Chemistry rests upon this foundation; and is guided in its researches and inductions, in its nomenclature and symbols, by a reference to this great principle. The doctrine of definite proportions is further the bond of 
connection between chemical actions, commonly so called, and the operation of those great natural forces, of which we have so often spoken as known to us through the material actions they induce. We are thus carried, under guidance of the strict laws of number, into parts of nature to which our senses give us no access, and which might well seem inapproachable by human reason.

In the preceding article, we have briefly noticed the manner in which the Atomic theory has been made to disclose to us these phenomena; to indicate the relative weights of the molecular parts of bodies, and the whole system of definite proportions of these molecules in every act of chemical change and combination. The method of proof is one equally simple and beautiful. It becomes more stringent and complete as we extend the number of bodies brought into evidence; and find the relative proportions so determined for each, strictly maintained in all their forms of combination. The conclusions are as certain as any of pure mathematics; or if there be seeming defect in any part, it is such only as may be due to imperfect analysis, or other causes not infringing on the truth of the general principle.

The corollaries of this great law are numerous, and all deriving certainty from the same source. The most important, perhaps, is the fact that compound bodies, as well as simple, have their fixed combining proportions; - the law here being that the combining number of a compound is exactly the sum of the combining numbers, or atomic weights, of its component parts. Closely akin to this is the further corollary, that compound bodies unite together in multiples of their combining proportions, as well as in single equivalents. And a further circumstance, of yet higher import to chemical theory, is the fact that bodies replace each other in combination in fixed equivalent quantities; so that in the mixture of certain neutral salts, if equivalents of each be brought 
together, the two bases exchange acids by an exact compensation; the original compounds are altogether lost and two new salts evolved, without either loss or addition of any kind in the process. So numerous are the facts and tables illustrating this doctrine of chemical equivalents, and so various the forms under which the subject may be viewed, that we must rest on this exposition of the principle, and proceed to other results from the law which is the basis of the whole.

One of the most curious of these has been derived from the researches of Berzelius into the chemical relations of the molecules of matter. This has received the name of Isomerism, as expressing the fact that in certain cases the same elements may be combined in exactly the same proportions, yet produce compounds having very different chemical properties. Two conditions of Isomerism may be noted; one in which the absolute number of atoms and consequently the atomic weight of the compound is the same:- the other, where, though the relative proportions of the elements are the same, the absolute number of atoms of each is different. But taking the simplest expression of the phenomenon, it necessarily implies a difference in such cases, in the mode of grouping together of atoms absolutely alike in nature, number, and relative proportion - a circumstance conceivable indeed, but never before proved. The whole investigation, still in its infancy, and perplexed by ambiguities, is of singular importance to the theory of compound bodies and to every part of organic chemistry. It promises for the future deeper insight into those primary atomic conditions of matter, which have yet been approached only by the rash speculator or imaginative poet.

The same may be said of another great discovery, analogous in kind, of Mitscherlich of Berlin; and to which the name of Isomorphism was applied, to express the fact it discloses; viz. that the chemical elements of certain bodies 
may be arranged in groups, so related together, that when similar combinations are formed from elements belonging to two, three, or more of them, such combinations will crystallise in the same geometric forms. Tables of these groups have been formed, and many of the results are exceedingly curious ; especially those which prove this peculiar isomorphic relation between various chemical substances, having in themselves other singular resemblances. Physical properties are thus associated by new relations; and methods suggested for hereafter discovering the common radical of substances now known only as distinct elements; an object ever before the mind of the philosophical chemist. Sulphur and selenium arsenic and phosphorus - lime and magnesia-chlorine, iodine, and bromine - are instances in their various forms of this curious connection of Isomorphism with other physical resemblances. The wide scope of the enquiry has led to various modes of arranging and viewing the results -of which the 'law of substitution,' suggested by Dumas, may be considered one of the most important.

Another topic, closely allied to the law of definite proportions, is the theory of that vast class of chemical compounds which we call salts - a vague name for a group of objects still imperfectly defined. The great complexity of this theory-enhanced by successive changes of doctrine and nomenclature - limits us to such mere outline as may suffice to show its nature, and bearing upon other parts of chemical knowledge.

There was a simplicity and seeming completeness in the old notion of oxygen as the acidifying principle - of the alkalies, earths, and metals as elementary bases - and of neutral bodies or salts as produced by their union, which made it difficult for chemists to acquiesce in any change of these views. But this became needful, when it was found that the most essential chemical characters of an acid might 
exist without the presence of oxygen, and that the alkalies and earths, with one exception, are compounds of this very element with metallic bases. When, further, the discovery of the new elements of chlorine, iodine, bromine, and fluorine, multiplied the classes and distinctions of salts, the whole theory became involved in a net-work of new names and divisions, which it was an arduous task to unravel. Without speaking of the oxygen, hydracid, sulphur, haloid, and polybasic salts (names which, as well as the word salts itself, may best be viewed as provisional phrases only), we must simply record our belief that Chemistry is here still far short of the point it is destined to reach. The true road, however (first indicated by Davy in his enquiry into the nature of chlorine), seems now fairly laid open by the researches of Graham, Liebig, Hofmann, Dumas, and other great chemists of our own day. Water, in itself, and in the two elements composing it, forms the foundation of the new doctrine. This wonderful fluid, so widely diffused in nature, comes into direct operation in almost every act of chemical change. It was already known that its presence is essential to the crystallisation of many bodies, and to the developement of certain acids. It was further known that water is always separated in fixed quantity, when an acid combines with alkalies or other bases to form a salt. But these observations long remained barren of result. It is only of late that chemistry, in recognising hydrogen as essential to the constitution of a free acid, has shown why water is necessary as furnishing this element. In proving, further, that in the combination of acids with alkalies or metallic oxides, the hydrogen is displaced by an equivalent of the metallic base, and combines with the oxygen set free from the latter, it shows the origin of the water separated in the formation of neutral salts.

What we thus briefly state, is the foundation of the modern view of the constitution of acids and salts - a 
doctrine still imperfect or faulty in parts, yet so far verified by tests, and simplifying so much the relations of these very numerous and complex bodies, that we cannot but admit a strong presumption in its favour when compared with all prior views on the subject. If the theory be not what is true, it seems at least in close kindred and approach to it. None, however, but a chemist can understand the difficulty and ambiguity of these questions - the douiblesidedness of all the objects and relations involved in them. Schemes and hypotheses have been altered to meet exigencies, under the same compulsion which created the crystalline spheres and epicycles of the ancient astronomy. The history of Chemistry speaks so largely of revolutions of doctrine, even in the vital parts of the science, that it would be hardihood to accept any one as final and complete.

Closely connected with, and dependent upon, the same great law of definite proportions, comes in the subject of Organic Chemistry; presenting in its progress and attainments one of the most signal triumphs of physical science, and promising future results which in endless ways may affect the welfare of mankind. No part of Chemistry so wonderfully expounds the power which has been gained over the elements of matter by the methods of analysis and synthesis, submitted to strict numerical relations even in the most complex compounds which nature or art place before us. A still higher interest belongs to the subject as the Chemistry of Life and of vital products; - of matter, whether animal or vegetable in kind, organised under that mysterious power, which has been termed the vital principle, in default of other understanding of this wonderful operation of Providence in the world. And yet further is it interesting, as extending research to the numerous artificial compounds obtained from the decomposition of these substances; a field of enquiry prolific of curious and profitable results. It may be that no 
absolute line of distinction exists between organic and inorganic chemistry; but, nevertheless, this division seems practically justified by the diversity of the objects they respectively offer to us.

Of more than sixty undecomposed elements now known, and which enter into the combinations of inorganic nature, sixteen may be numbered as more or less essential to the products of organic chemistry. Among these, however, the four elements of carbon, oxygen, hydragen, and nitrogen are so largely predominant in quantity, if not more essential in their presence, that to them the name of Organic Elements may especially be applied. Every organic compound contains three of these elements - a large proportion, especially among animal substances, all the four. The other elements concerned in Organic Chemistry (including sulphur, phosphorus, chlorine, iodine, the metallic bases of the alkalies and earths, iron, \&c.), though comparatively of small amount, yet appear to be necessary to the organised bodies in which they are severally found. Out of these various materials, but mainly from the four first mentioned, are formed the countless combinations which the chemist has subjected to his science; - analysing those which nature has so profusely presented in the animal and vegetable world; - forming new compounds by intermixture and varied proportions of these elements; - and in some cases, by a still higher art, even producing certain of those organic compounds, which were known before only through the occult chemistry of living beings. We should despair of giving those of our readers who are new to the subject, any adequate idea of the vast labours, and not less vast results, which illustrate this department of the science. Scarcely is there a principle or product of organised existence which has not been submitted to rigid examination, and tried in all its relations of affinity with other bodies; and nomenclature has been taxed to its 
utmost power, to record the results derived from this great scheme of systematic enquiry.

While still compelled to limit ourselves to a mere outline of these methods and results, we must briefly notice their connection with those discoveries of Liebig in animal and vegetable chemistry, which have received such important application to physiology, medicine, agriculture, and other arts of life. These applications - (in which, as well as in the labours that led to them, the names of other enquirers are largely associated) - give to the subject of organic chemistry its peculiar colouring and character. It is a science eminently practical in relation to the physical interests and necessities of man. In analysing and otherwise examining the various solids and fluids which enter into the fabric of animal life - and in submitting to similar experiment the ingesta of aliment and air which minister to its growth and preservation, and the egesta which provide for the perpetual and necessary change of parts - this branch of Chemistry becomes a main pillar of physiology, and offers the fairest hope we can entertain of raising medicine to the rank of the more exact sciences.* While by researches of equal exactness, directed to vegetable substances; and to those elements in the atmosphere, in soils, and in manures, which serve to their nutriment and various properties, agriculture is made to assume the character of a science, and Man obtains new and more definite dominion over that earth on which it is his destiny to labour for existence.

All alimentary substances, in fact, whatever their nature,

* We may take as one instance of these refined analyses, that of coffee by Payen, who, in this familiar berry, discovers and denotes ten different ingredients, all existing in determinate proportions. Caffein itself (the ingredient which gives its peculiar property to coffee) is a compound of the four great organic elements in fixed proportions, and its chemical relations to other bodies offer some of those complex names in which Chemistry abounds-Dimethylalloxantin, Caffeo-murexid, \&c. 
originate in vegetable growth. Upon this fact was founded the inference that, by the processes of vegetation, inorganic materials are converted into organic compounds; which, serving again as food to animal life, create a new class of organic products fulfilling higher purposes in the economy of the world. This view, plausible in itself, has merged in the later discoveries of Liebig, Mulder and others, which prove that not merely the saccharine and oleaginous principles of animals and vegetables are almost identical in chemical composition, but that even the three great principles of animal tissues - albumen, fibrin, and casein - have their exact counterparts in certain of the principal products of vegetable life; the proportions of carbon, oxygen, nitrogen, and hydrogen being precisely the same in each. This discovery, startling by its unexpectedness, was followed by attempts at yet larger generalisation, as in Mulder's doctrine of Proteine; but these have not succeeded in attaining the proof which science requires, -and go no further than to show the difficulty of interpreting the realities of nature by our artificial systems.

Connected closely with this topic, and better established as a principle, is the important doctrine of Compound Radicals, already mentioned as one of those great general views which especially mark the present æra in science. Liebig has even described Organic Chemistry as the 'Chemistry of Compound Radicals,' and in this definition there is much of truth. The term expresses a class of compound bodies, possessing a certain unity and stability of composition, through which they fulfill every part of simple bases; - uniting as such, not only with elementary bodies, but with each other; and generating large classes of secondary products, which have all relation to the compound radical thus assumed as a base. Some of the compounds thus characterised have been obtained in a separate state - as Cyanogen, for example, in 
which two atoms of carbon and one of nitrogen combined act as a single atom or combining equivalent; - and the strange substance called Kakodyle, already described, in which carbon, hydrogen, and arsenic combine to form a radical, singularly marked and active in its affinities. Others of these peculiar bases were at first known only conjecturally, but their existence inferred from the analogy of the compounds they form. Ethyl, for example, the radical of the numerous class of ethers, was defined to us through all its various combinations before it had actually been obtained in a separate state. In naming the hydrate of oxide of ethyle, as the equivalent of alcohol in the new chemical phraseology, we at once illustrate the theory of these compound radicals, and the nomenclature which is needed to express their presumed character and relations.

It must be conceded that in this abstruse part of Chemistry various assumptions are made, which may be disproved by future research; and that among the numerous contingencies of combination, furnished by the complex series of combining bodies and proportions, the particular schemes now adopted may not be those which actually exist in nature. This uncertainty is shown by the various ways in which chemists have represented the grouping of parts in the same compound. It belongs, however, to the modes of union only; and in no way impeaches the truth of analysis, or the exactness of those laws of definite proportion which form the foundation of the whole. The doctrine of compound radicals occurs, indeed, as a sort of corollary from these laws. Chemical affinity, acting on the molecules of different bodies, with every degree of force, produces combinations infinitely various in stability. This is especially true as regards organic compounds; the simplest of which in aspect - as sugar, starch, albumen, \&c. - are composed in their smallest atoms, not of single ratios of the organic elements, but of multiple proportions of the 
atomic weights, definite for each particular substance, and giving to each its peculiar properties. It is a natural inference, confirmed by observation, that of these complex molecules thus differing in stability, some should run hastily into dissolution, others hold tenaciously together, so as to be capable of entering as bases into new combinations without losing their identity. From the latter condition we derive the probable theory of compound radicals; thus merging it in those great laws which govern all the chemical affinities that nature or art place before us.

The vastness of this subject of Organic Chemistry precludes those examples which might animate, as well as illustrate, our review of it. We would willingly adduce some instances of those beautiful series in which, whether we ascend the scale from the simplest, or descend from the most complex, we find organic compounds, infinitely various in properties, produced by the simple addition or subtraction of elementary atoms; - each such change in the series defined by strict numerical relations, and capable almost of being expressed by algebraic symbols. A striking example we have in that remarkable series which ascends from olefiant gas-the simplest atomical union of carbon and hydrogen - through various intermediate chemical compounds, to sugar as the summit of the scale. Each successive step here renders the arrangement of atoms numerically more complex; but all are so submitted to definite proportions, that the chemist, in dealing with these substances, can predicate exactly what number of atoms must be added or removed, to effect each successive change from one to another. The series of products obtained from common Coal is another striking example of these relations. To naphtha, as the first product, succeed benzule, nitro-benzule, aniline, and rosaniline; - the latter yielding, by simple addition of water, that Magenta dye which now gives such splendid colouring to the various tissues from our looms. 
And while coal thus ministers even to the most delicate works of art, a fetid oil (Fusel Oil), obtained during the distillation of spirits, is made by the refinements of chemical process to afford all the delicate odours of the most fragrant fruits. These transformations are the achievement of the Organic Chemistry of our day.

In the system of classification by homologous series we owe much to the various labours of Gerhardt, and to those which Hofmann is ever so successfully prosecuting. The question as to the nomenclature best adapted to express these series, is still beset with various difficulties. The present scheme (mainly founded on the views of Berzelius and Gerhardt), though well fulfilling all important practical objects, may probably be considered provisional only. In the case of Chemistry very especially, nomenclature becomes almost a science in itself; such and so complex are the objects it is called upon to denote.

A wonderful part of the phenomena of Organic Chemistry is the diversity of properties produced, even by slight changes in elementary composition and proportions. We have already noted this in certain instances; but the proofs, most singular and impressive, are those connected with the influence of organic agents on animal life. An atom added to or abstracted from a compound, determines whether the product be wholesome or noxious - an aliment or a poison. So closely is the Chemistry of the material world around us associated with that still more refined and mysterious Chemistry, which ministers to the phenomena of life! Every solid tissue, every fluid of the body, has its appropriate chemical composition and relations. Every organic function either depends upon or involves chemical changes in its progress. The air we breathe is no sooner within the lungs than these changes begin; analogous to combustion in their nature, and effecting that transformation from venous to arterial blood, which is 
essential to life in its every part. The food we take hardly enters the stomach before it becomes the subject of chemical actions, which are continued and multiplied, till its final assimilation and admission into the mass of circulating fluids. All the secretions and excretions from the blood, many of them singularly complex in nature, depend on like agency; subordinate, however, as is all besides in the animal frame, to that vital action which we everywhere see in its effects, though unable to separate or define it. Morbid changes and growths may frequently be referred to the same actions, abnormal in kind; and we have cause to believe that, under deficient vitality, either from disease or old age, these purely physical processes do often so usurp upon the fabric and functions of life as to become the immediate causes of death. Equally may we believe, from recent researches in physiology and pathology, that certain diseases have their origin in chemical changes of the blood; either generating morbid agents within itself, or multiplying, by an action analogous to fermentation, poisons ànd morbid matters received into the body. This wonderful fluid, ever in motion and change, and subject at once to chemical laws and to the principle of life, is in itself a mine of future discovery; not to be worked otherwise than by consummate skill and perseverance, but well meriting the application of both.

We have spoken of actions analogous to fermentation; and are thence led to notice shortly another great attainment of Organic Chemistry in regard to the remarkable process so named, and the kindred changes of putrefaction and decay. All these depend on chemical decomposition, as it occurs in organic compounds, and especially in those of which nitrogen is a principal constituent. They make provision for that constant succession in plants and animals, which is the condition of organised existence on the earth. Carbonic acid, ammonia, and water are supplied by the atmosphere as the 
elements of vegetable life, and of animal life as derived from it; - the same three compounds are rendered back as the ultimate products of fermentation and decay. Liebig and Berzelius have deeply studied these processes; and the theory of fermentation proposed by the former has found general adoption. It is based on the curious fact, of which Chemistry is prolific in examples, that a body in a state of intestinal motion or change among its particles may, by mere contact, produce in another body analogous changes, decompositions, and new products - and this, though the exciting body be infinitely small in proportion, and yields none of its own elements to the products evolved. The importance of this doctrine of ferments will be readily understood. It bears upon various familiar phenomena everywhere around us, but still more strikingly on the whole theory of matters which act as poisons on the living frame. We have scarcely got beyond the threshold of this great mystery. It is easy to quote the rapid growth of fungoid life; and not impossible in theory to conceive that a minute particle of morbid matter received into the body may gradually evolve the phenomena of small-pox or other zymotic disease. But beyond this comes the stranger fact of that permanent change induced on the body by certain diseases; a change which, while unseen in any other function of life, is so wonderfully attested by immunity from future action of the same morbid cause. No atomic or other theory can reach this phenomenon, which still lies hidden among the arcana of life itself.

The same cursory view we must take of another signal attainment of Organic Chemistry, viz. the formation artificially of various organic bodies, both of animal and vegetable kind, not merely analogous, but identical with those presented to us in nature. The number of the several substances thus produced now exceeds twenty; but does not yet include any 
organised tissue, or the substances, albumen, fibrine, gelatine, \&c., out of which such tissues are formed. Further, it must be stated that the products thus artificially obtained are not derived directly from the simple inorganic elements, as we know to happen in the original processes of nature, but are formed by change and new combination from other organic compounds. The distinction is obviously an important one, though it may be that the progress of science will hereafter lessen its value, and bring organic and inorganic chemistry into still closer union than they have yet attained. We are here, however, approaching the margin of that gulf which separates physical facts from the proper phenomena of life; and it is possible that the intervention of vitality as an element of action may arrest all ulterior progress in this direction. Though it would be rash to draw the line of demarcation too closely around us, the limit must somewhere exist; and even should synthetical chemistry succeed in producing the materials of organised tissues directly from their elements, we have reason to believe that here its power will stop. The actual formation of these tissues is doubtless due to the intervention of that higher generative process, the operations of which we see and designate by name, but cannot hope to reach or imitate.*

The outline, we have given of Organic Chemistry can afford

* As connected with the subject of this paragraph, we may notice the striking discovery of M. Berthelot, made known after the first edition of this volume was published. A beautiful method of experiment enabled him to obtain one of the hydro-carbonous compounds, by direct union of its two elements, - the electric current between the carbon poles being made to pass through an atmosphere of very pure hydrogen, and the union taking place in the space thus intensely heated. To the chemist this result is one of great interest. Acetylene, the product obtained, consists of four atoms of carbon with two of hydrogen; and is one of the simplest of a series of compounds, on which the processes of Chemistry are continually engaged. Olefiant gas is easily derived from it; and this again yields alcohol to a further operation;-the whole illustrating admirably those subtle researches through which science is now exploring all the phenomena of the material world. 
but a slight idea of the magnitude and variety of these researches. Scarcely have we even named the great class of vegetable alkaloids obtained by modern analysis; many of them most singular in their action, medicinally or as poisons, on the animal economy. Of the acids, at least 300 in number, which are described in this department of chemistry, we have spoken almost as cursorily. Arranged in different series, upon some predominant character of each (the alcoholic, aromatic, fatty series, \&c.), these organic acids strikingly illustrate the doctrine of definite proportions in its every part. In the fatty series for example, including more than twenty members, the oxygen is the same in all; the other constituents, carbon and hydrogen, preserving the same exact ratio of increase throughout the whole of the scale. Gaps, determined by the very exactness of this ratio, have been left here as in the other series. One by one the progress of discovery is filling them up; and under numerical conditions precisely those which the theory requires.

In every part of this picture of modern Chemistry it will be seen how wonderfully the objects cohere together; and amidst the immense multiplication of facts, and even of new classes of phenomena, how strikingly all converge around the law of definite proportions in combination - the keystone of Chemistry, and the index to all the actions involved in it. The name of Liebig, as we have seen, is ever prominent in this narrative. If there were license for comparison in things so different, we should be tempted to call him the Rubens of the chemical school, from the power he possesses of boldly grouping together his results, and enforcing conclusions which have escaped more timid reasoners. Yet while thus successful in reaching great truths - felicissime audax - we are bound to admit a certain rashness which now and then hurries him into inferences not tenable under 
more exact knowledge; - a fact especially to be noted in some of his applications of chemistry to animal physiology. It is the single deduction we have to make from the merits of his various labours.

Had we space for it, many other attainments of modern Chemistry might be named, all hanging upon the same great principle. Such are the relations of combining volumes of gases to their atomic weights; - the peculiar phenomena of catalysis and of nascent gases ; - the observations of Dulong, Petit, Neumann, \&c., on the relation between the specific heat of bodies and their atomic weights; - the facts connected with allotropy and the curious mutual actions of like atoms, well illustrated by Brodie; - and the bold speculation of Dumas, representing what may be termed chemical atoms (those which enter primarily into chemical combination) as themselves made up of groups of molecules, and divisible into sub-multiple parts; - an hypothesis suggested to meet difficulties as to the Hydrogen unit in the scale of Atomic weights.

A more particular mention is due to the remarkable researches of Mr. Graham (Master of the Mint), which may almost be said to offer a new mode of Chemical analysis to our knowledge. Begun twelve years ago by experiments on saline liquids and on gases, these reseaxches have lately been much extended, and their principle developed in a paper presented to the Royal Society (Nov. 1861), "On Liquid Diffusion applied to Analysis." The term of Dialysis might fitly be used here, since the actual agent in separation of the constituents of a chemical solution is simply wellsized letter paper, through which permeation takes place. This paper is not porous enough to act as a filter, or give passage to the mixed fluid as a whole; but it transmits certain molecular parts of these solutions, and thereby effects a real chemical decomposition, by means almost mechanical 
in kind. The simplicity of the method in nowise lessens the value of the results obtained. Mr. Graham has already drawn from this enquiry some important distinctions as to the physical properties of the substances acted upon, and as to the part which the water itself bears in this phenomena. It is a new path to knowledge, but certain hereafter to coalesce with the many others which have the same object before them.

If this were a treatise on Chemistry, instead of a mere outline of the present progress and objects of the science, it could not be concluded without an ample review of those great discoveries of the last fifty years, which depend upon and illustrate the relations of Electricity and Light to Chemical action. The subject, however (already touched upon in some preceding articles), is far too large for our limits here; and we can notice it only so far as to show the important place it holds as a part of physical science.

The decomposition of water by voltaic electricity, with disengagement of oxygen and hydrogen at the opposite poles, was the great initial fact which associated chemical and electrical phenomena. Further experiments extended this law of decomposition to other compound bodies, placed in solution in the electric circuit; - certain of their compound parts always appearing at the positive pole, others at the negative. The admirable researches of Davy, in 1806-7, enlarged the scope of these results, by showing that chemical agents under this condition of electric polarity were seemingly transported freely and unaltered from one pole to the other; though made to pass through intermediate vessels, containing chemical agents with which in ordinary circumstances they would instantly and forcibly combine. In sequel to these experiments, and by the same agency, Davy succeeded in obtaining the metallic bases of the alkalies and 
earths; - a discovery more imposing on first aspect, yet of less real import than that great principle of electro-chemical action of which it formed a particular result.

From the period just named to the present time, the development of this principle and its extension to new objects has been continued with unceasing activity. It is well known how greatly the genius and labours of Faraday have contributed to this success, not solely by the discovery of facts, but by the determination of the laws which govern. them. We take but one example in point. Earlier opinion had supposed a peculiar energy of the poles in an electric circuit, determining the chemical actions which manifest themselves at these points. Faraday regards the poles as simply opening a way or passage to the electric current; and looks to the Electrolyte - that is, the fluid chemical compound interposed between them - as the source and medium of the chemical changes going on. These changes consist (and here lies the important point) not in any actual transference of material particles, but in a series of successive decompositions and recompositions in the line of particles between the poles, evolving the component parts of the electrolyte only where the current ceases to flow through it. This may seem at first sight a strange complexity of action. But it will not so appear to those accustomed to regard the atomic relations of matter, as they must necessarily exist to fulfill the various conditions of chemical change already known to us. And not of chemical change alone, strictly so termed, but probably of all changes taking place in the material world. We reach the greatest phenomena of the universe through the very actions of molecules or atoms, the minuteness of which transcends all human conception.

The true theory of the relation between these two great elements of force or power in the material world-electricity and chemical action - has been, and even yet is, a qucestio 
vexata among philosophers. The solution of the question, when obtained, will doubtless equally apply to and illustrate both. The opposite opinions of Volta and Wollaston, as to the priority of action in these powers, were not reconciled by Davy's researches. But Faraday has given confirmation to Wollaston's view, and a more just expression of the general theory, by proving that no chemical action can ever occur without development of electricity; and, conversely, that the electrical force is never put into activity without some evidence of chemical change. Thus regarded, the whole subject becomes subordinate to that great law of the Co-relation of Forces of which we have so often had occasion to speak.

Still more briefly must we notice the relations between chemical action and light. Their illustration through photography in its various forms has now become familiar to all. If seeking another and less familiar example to denote these subtle relations, we might find it in the curious influence of solutions of sugar on polarised light passing through them, and the variations of this influence by simple changes of temperature. The test thus obtained is delicate enough to show the particular quality, as well as quantity, of sugar in solution, by the amount of deflection or rotation it produces in the polarised ray. An instance of this kind carries its interpretation far beyond the simple limits of the experiment. It is an index of relations which pervade all nature.

This short history of Modern Chemistry we must now bring to a close; though, in so doing, we omit many other discoveries which have given lustre to the period under review. Even in this outline, however, enough has been stated to justify our assertion of the marvellous progress of the science, and our anticipation of future results hardly less remarkable than those already obtained. We have been careful to indicate, as the subjects came before us, the probable paths of 
future discovery; each giving access to new truths, yet all converging towards common principles and powers. If there be less of epic character here than in Astronomy, yet does the science of Chemistry form a noble didactic poem admirable and harmonious in all its parts, and carrying us forwards, through a long series of wonderful phenomena, to those great and eternal laws which express the Providence and the wisdom governing the world. 


\section{NATURAL HISTORY OF MAN.**}

[QUARTERLY REVIEW, No. 171.]

THE subject before us can never be out of season or date 1 as long as Man has his place on the earth. For what enquiry of higher import than that which regards the physical condition of the human species, as first created and appearing on the surface of the globe? What investigation in natural science of deeper interest than that which, from study of the numerous races and physical varieties of Man, and of the equally numerous diversities of human language, draws conclusions as to the sources whence these wonderful results have been derived, and as to the manner and course of their developement? Questions like these, even if already settled to our reason, would yet have a constant hold on the minds of all thinking men, in their simple relation to that greatest of all phenomena - the existence of human life upon the earth. But, in truth, they are far from being thus settled. A spacious field is open to research, in which certain paths are laid down, and certain landmarks fixed in

* 1. Researches into the Physical History of Mankind. By James C. Prichard, M.D., F.R.S., Corresponding Member of the National Institute of France. Third Edition. 5 vols. 8ro. 1836-1847.

2. The Natural History of Man; comprising Inquiries into the Modifying Influences of Physical and Moral Agencies in different Tribes of the Human Family. By the same. 1843.

This article has been much abridged, and the philological part of the argument wholly omitted, except in the denotation of its importance to any complete work on the subject. 
guidance; but where complete knowledge has not yet been reached, and where even the boundary of what may hereafter be effected by human effort is still obscure.

We are justified in presenting this subject to our readers, even in a very abridged form, from the conviction that the great questions it involves are still only partially appreciated by those familiar with other branches of science. The history of Man, as a denizen of the earth, has indeed been conceived and pursued in many different ways, according to the objects, genius, or opportunity of those engaged in this interesting study. But these portraitures which have severally represented him as

The glory, jest, and riddle of the world,

are partial and subordinate, and in nowise fulfill the purport of the larger title before us. The philosopher living in the comparative seclusion of one community, may indeed (as Blumenbach and Prichard have done) construct a science from the labours of those cosmopolite travellers who have studied mankind on a bold and broad scale, under every diversity of region and race. But, generally speaking, the tendency of common life and habitual pursuits in the more civilised communities is to narrow, by division and refinement, all great views of the human race. The social pictures of Man found in poetry, history, essay, and romance, will explain our meaning. They are for the most part individualities of character or custom, which tend rather to curtail than enlarge the enquiry, and have little relation to the Natural History of Man as a part of creation at large. Even moral and religious feelings are concerned in giving their tone and temper to such investigations, differently defining the objects and pursuing them by separate routes. And further, these objects are in themselves so numerous, and their aspects of such endless variety, that we can scarcely wonder at the vague 
understanding of the questions which lie at the bottom of the whole - questions well worthy, nevertheless, of all the learning and ingenuity given to their solution.

Whatever may be the causes, certain it is that the physical history of Man has only recently taken its place as a definite branch of science. The ancient philosophers dealt with it loosely and erroneously. Their limited knowledge of the surface of the earth - their ignorance of whole existing races of mankind - the prejudices of their mythology - and their imperfect understanding of scientific evidence (the preference

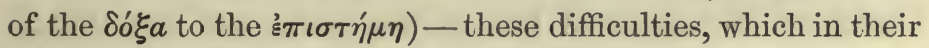
totality even the genius of Aristotle could not surmount, will readily explain the fact we have stated. Passing over the earlier researches of Camper and others, we may affirm that the true foundation of the science was that laid by Blumenbach of Gottingen, whose long life of honourable labour closed not many years ago. His celebrated collection of skulls (which we have ourselves examined under his guidance), obtained by unwearied perseverance from every part of the globe, suggested new relations and more exact enquiries in prosecution of one branch of the subject. The researches and writings of Cuvier, Humboldt, Lawrence, Owen, Tiedemann, Rudolfi, and other physiologists, while differing in certain conclusions, have continually enlarged the scope of the science and concentrated the results obtained by travellers and naturalists; - thus augmenting the means upon which the removal of these differences and the certainty of all conclusions must eventually depend. Philology, meanwhile, has come largely in aid of the enquiry, and the study and classification of languages, indicated more remotely by Scaliger, Bacon, and Leibnitz, has grown into a vast body of authentic knowledge, ministering through new and unexpected relations to the history of the races and communities of mankind. The names of Adelung, Schlegel, Remusat, 
W. Humboldt, Grimm, Bopp, Klaproth, Rask, \&c., indicate the more conspicuous of those who have advanced this science abroad. In our own country we may enumerate Harris, Horne Tooke, Sir W. Jones, Wilkins, Marsden, Young, Prichard, Latham, \&c., as associates in the same learned career. We gladly too annex the eminent name of Max Müller to the latter list.

What, then, are we to understand by the title prefixed to this article? In stating it to be the natural history of Man, as a branch of that larger science which includes the physical history of all organised life on the globe, we give but a meagre conception of the subject. Vegetable life, individually fixed to a single spot-generically distributed into different regions so as to form a science of botanical geography - limited by climate, soil, and other circumstances, though capable of vast changes by culture - all this, while furnishing much curious illustration and analogy, does only slightly represent to us what pertains to the physical history of the human race. When we rise in the scale of creation through the innumerable forms and gradations of animal life, and reach those wonderful instincts and yet higher functions of intelligence and feeling in some animals, which Aristotle calls $\mu \iota \mu \dot{\eta}_{\mu} \alpha \tau a \tau \hat{\eta} s \dot{a} \nu \theta \rho \omega \pi i v \eta s ~ \zeta \omega \hat{\eta} s$ - though finding some of the analogies to approach more closely, still we are far below the level of those great questions which regard the human species, viz. the origin, dispersion, and mutual relation of the various races of mankind. To mere physical evidence are here added other and higher methods of proof, connected with the exercise of those mental faculties which mark Man as the head of the animal creation. The peculiarity, the grandeur, and, we may add, the difficulty of the theme, depend mainly on his condition as an intellectual being, whereby his whole existence on earth is defined, and his relation to all other parts of created life. 
The question as to the singleness of origin of the human race may be said to govern the whole subject; since if this fact be admitted or proved, many subordinate questions. are settled at once. If, for instance, it should be rendered certain to our belief that all mankind - throughout all ages of human existence on the globe, and in all their innumerable varieties of form, colour, customs, and language - had been derived from one single pair, nothing remains but to investigate the causes, physical and moral, which have produced from this unity of origin the wonderful diversities everywhere visible. A subject wide enough in truth to satisfy the most eager speculator! yet well defined in its limits, and even in many of the paths through which research must be pursued. But this simpler form of the question is not permitted to us. The point is one upon which naturalists of eminence have held very different opinions. It has been contended not only that there is no proof of the derivation of mankind from a single pair, but that the probability is against it. Some have ventured to suppose an original and absolute difference of species. Many have adopted the idea of detached acts of creation, through which certain of the more prominent races had their origin in different localities; - interblending afterwards, so as to give rise to those subordinate varieties seen so numerously around us. Others again, putting aside the notion of the immutability of species, have boldly hazarded the belief that inferior animal organisations, either fortuitously, or by necessities or latent laws of nature, may have risen into the human form: and this under conditions so far unlike, as to give origin to those varieties which have perplexed our ideas of unity, and puzzled both philosopher and physiologist to explain.

Before going farther, we may briefly advert to a point which must already have occurred to every reader. Has not this question been long ago settled on the authority of 
Scripture, so as to preclude all further discussion upon it? Are we entitled to go beyond, and to risk any portion of our faith upon statements or inductions derived from other sources, if contradicting the interpretations commonly given to this higher authority?

The question is one not new to modern science. In reply to it, and to vindicate that right of reason and enquiry which Man has received as one of the greatest gifts from his Creator, it might be enough for us to cite passages from the writings of several distinguished geologists, who have weighed this point with all the seriousness and candour befitting their reputation as men of piety and truth. The difference of the subject here does in nowise affect the argument, which applies alike and with equal force to both cases. Take indeed what course we may, these questions, from their very nature, must needs infix themselves deeply in the minds of thinking men, and become in one form or other the matter of earnest enquiry. That the cause of truth will assuredly gain in the end, we can affirm with the greater satisfaction in this case, because it is our conviction that the conclusions of reason and science, unaided by Scripture, concur mainly with that derived from the latter source. While admitting the difficulties inherent in the subject, we think there are sufficient grounds for believing that all races and diversities of mankind are really derived from a single pair; placed on the earth for the peopling of its surface in the times before us, and during the ages which the Creator may yet assign to the present order of existence here. The arguments for such belief we shall now state; endeavouring to place the evidence in as clear a form as possible, and to aid those unacquainted with the subject in comprehending the relative value and effect of the proofs so given.

What then are the sources of knowledge, what the methods of research, through which to attempt the solution of this ? 
question? They may best, we believe, be classed under three heads : - First, the Physiological; including all that regards the physical conformation of Man - his mental endowments - the question of the unity or plurality of species - and the laws which license or limit the deviations from a common standard. Secondly, the Philological; including all that relates to human languages - their connections, diversities, the theory of the changes they undergo, and the history of such actual changes, as far as we can follow it. Thirdly, the Historical; including all written history, inscriptions, traditions, mythology, and even the more common usages which designate and distinguish the different communities of mankind.

This too seems the natural course and order of the enquiry. Man is first to be considered as a part of the animal creation at large, and under the many points of close and unalterable likeness to other forms of animal life, in all that relates to his procreation, nutrition, growth, decay, and death; - as well as in regard to the modifications of which the species is susceptible, and the diversities it actually exhibits. Various instincts - belonging especially to the early stage of life before the higher faculties have risen into action - further attest this great natural relation, which human pride can neither deny nor discard. But beyond and above this comes in the peculiar condition of Man as an intellectual being, richly provided by his Maker with those endowments which, in their highest elevation from nature or culture, have bequeathed to the admiration of all ages names made immortal by their genius and attainments - (Homer, Aristotle, Dante, Bacon, Shakspeare, Milton, Newton, Leibnitz, Pascal, Laplace, and others which crowd on the memory) - and gifted yet further with that moral sense, those faculties and sensibilities of feeling and passion, to which, duly guarded and governed, we owe our understanding of virtue and 
conscience, and of all that is beautiful and sublime in the world around;-forming what Milton has well called 'a piece of divinity within us; something that was before the elements, and owing no homage to the sun.'

The consideration of these higher attributes of Man, and of that faculty of speech, which more than any other marks his place and destiny in creation, carries us naturally to the second, or philological part of the enquiry. Human language is becoming one great index to the history of mankind. Its numerous forms, as we find them in existence and maturity among different communities of men - forms, in many cases so remote in the roots of words, in grammatical structure and idiom, that the doubt might well arise whether they can have any common origin - these very diversities, as well as the connections of languages, are all subservient to the enquiry before us. We have already spoken of the many eminent men who have devoted themselves to this part of the subject; collating on philosophical principles the numerous languages which crowd the globe; and giving to the history of races and nations, irrespectively of all other tradition, a new and wider basis than heretofore. The progress of such researches of late years is the best exponent of what may be attained by their future prosecution.

To the physiological and philological succeeds the historical part of the argument. It might seem on superficial view that this would be the most copious source of knowledge as to the physical history of Man, and his original dispersion over the earth. We might expect here to verify and extend the conclusions derived from the former methods of enquiry, and to give to the whole more certainty and completeness. And so it is, whenever we can obtain concurrence, or even approximation of results, from these different sources. But, pursuing the investigation on this ground alone, we shall find ourselves speedily and continually at fault. History, as we 
have it in our hands, is rarely capable of conducting us to the solution of this great question, seen dimly through the mists of time, and often rather obscured than enlightened by human tradition. The line, variously broken and interrupted before, stops where the more arduous part of the enquiry begins, and gives us no guidance into the ages beyond.

We might much enlarge, were it needful, on this incapacity of History to satisfy our just curiosity as to the primitive condition of the human race on earth. But we shall confine ourselves to a few general remarks, such as may obviate misconception as to the value of this part of the evidence. In placing them here, we deviate from the order just laid down; but we do this purposely, that attention may be better concentrated afterwards on the other topics, on which the solution of the enquiry chiefly depends.

We have already spoken generally of the bearing of sacred history on this subject. In the Old Testament we have a record of the creation of Man upon the earth, and of a line of successive generations down to the period of the Deluge; from which we are taught to date a second growth and dispersion of mankind. But it would wrong the proper objects and influence of Scripture to regard it as a physical history of Man, or to seek in its pages for the facts with which this science has especial concern. A few passages only can be brought to bear directly on the conclusions we seek to obtain; and there is constant danger, as well as difficulty, in tampering with words and phrases so alien in their objects and manner of use. The Mosaic writings are the record of the origin and progress of one people, wonderful in every age of its history, and by the dispensation of Providence signal in its influence on the human race. All that is given to us, apart from this main object, is incidental, brief, and obscure. The chronology of the Jewish people itself is rendered ambiguous by.the recognised differences of 
time as recorded in the Hebrew, Samaritan, and Septuagint texts; amounting altogether to a period as long as that which has elapsed from the beginning of the Anglo-Saxon monarchy to the present day. Even in an early part of these books we find allusion to nations which had grown into existence and power; but without any sign to mark their origin, beyond some single name, or the general statement of the multiplication of Man on the earth. It is, however, the affirmation of the origin of mankind from a single pair of created beings which forms the link between the Scripture narrative and the subject before us. We must not look to it for description of the primitive physical characters of the human species, or for details as to the origin of human languages. But it is much to arrive at the same point through paths thus diverse; and we shall do well for the cause of truth to hold the sacred volume ever in our hands, seeing where it fairly comes into contact with other knowledge, but never forcing its peculiar objects and phraseology into conclusions with which it has no concern.

Passing from the Scriptural to other history, - whether of writings, tradition, or mythology, - we lose this distinct affirmation of the unity of mankind, without gaining any more certain record of the primitive state of the species. The notices indeed multiply as to the growth and spread of particular tribes; but even if possessing more authority than belongs to them, they go short way towards giving us knowledge of that mysterious period, which intervenes between the creation of Man and the formation of nations and empires. We lose ourselves in utter darkness when we seek to go beyond certain epochs, remarkable in the ancient world as the period of great movements and migrations among the people best known to us. One of these may especially be denoted, as comprising within a very short time 
the record of six migrations and settlements, each containing some germ of future history.* Yet even this period, in which were sown the seeds that ripened into Grecian genius and civilisation, how vaguely and scantily is it known to us! How much more obscurely still those vast Celtic, Teutonic, and Sclavonic migrations which have given cast and colour to all the succeeding destinies of Europe! Here we have hardly the ground of tradition to stand upon: all measure of time is lost; and we are obliged to come at once to the relations of language, as the only index we possess to these mysteries of the ancient world.

Of the grandeur of Egypt at a remote period we have numerous proofs; and the genius and industry of the present age have derived from its sublime monuments, its hieroglyphics and paintings, the evidences of vast extent of power, of various refinements of policy and civilisation. But in this very point is seen the deficiency of history. Whence, and how, this growth of grandeur, unrevealed in its origin, and so faintly traced in its earlier progress? Long series of sovereigns have been determined through hieroglyphic inscriptions, compared with the fragments of history; - the founders and dates of many of the great monuments similarly fixed; - certain astronomical periods ascertained; and a chronology of some exactness carried back to a remote antiquity. But antiquity is only a relative term; and the researches of Bunsen and Lepsius, the latest labourers in this field, though stretching backwards nearly 5,000 years, are arrested at a period far short of the origin of the remarkable nation on whose history they have bestowed so much learning and toil.

The history of the Assyrian Empire, contemporary with

* Cuvier has particularly marked this period, extending from about 1550 A.C. to 1450 A.C., and including, besides the departure of the Israelites from Egypt, some of the most noted epochs of settlement in Greece. 
that of Egypt, has been more deeply sunk in obscurity. Fragmentary notices in Scripture and in Greek authors have told us of its greatness and conquests, the magnitude and decorations of its capital. But we have only just begun to disentomb these great cities, and can but partially decipher the cuneiform characters which designate and give date to their wonderful works of art. The vast empires of China and India offer the same striking examples of this imperfection of history, as bearing on the early condition and diffusion of the human race. Native records, aided here also by astronomy, carry us obscurely back to dates as remote as those of Egypt and Assyria; but beyond this all is lost in the depths of time, or in the still darker depths of mythology. And to take another instance, from a different source but not less cogent for our object, Where do we find the faintest authentic trace of those maritime migrations, seemingly not single but successive, which peopled the great American continent; giving birth to numerous nations and languages, and to various monuments of power and partial civilisation? Here only one or two vague traditions float before us, which poetry may adopt, but which history refuses for its more definite and graver purposes.

These few examples will show how scantily we can draw from ancient history the information required. We nowhere get high enough. The regions of tradition or mythology are reached; but it is still the selva oscura, the basso loco of the poet, and we do not obtain access beyond. It may even be affirmed that less certain knowledge of the early races of mankind is derived from direct history than from those resemblances of custom which often remain infixed for ages, when all other connections are lost. Such are the usages pertaining to birth and death - the methods of warfare the regulations of property - the punishment of offences the manner of habitation - and yet more remarkably the 
bodily mutilations which are found so strangely to exist in common among races widely separate on the earth. Much caution indeed is needful in dealing with these indications. There is the same liability to deception here as in the case of etymology, where ingenuity so often deceives itself by a shadow of resemblance. But pursued with discretion and the multiplication of authentic facts, this method of research becomes fruitful of curious results; and, like those branches of the enquiry to which we are now hastening, gives yet more abundant promise for the future, aided as it is by numerous facilities, unknown and unsurmised heretofore.

We have dwelt thus long on preliminary parts of the subject, under the conviction that many, even of those conversant with other sciences, scarcely appreciate the entire scope of that under our review. We come now to the two main sources of knowledge regarding the natural history of Man, viz. human physiology and human language; lines of argument distinct in themselves, yet parallel in direction, and mutually giving force to every conclusion in which they occur. Through these channels alone can we proceed upwards when history and tradition alike desert us. Even admitting that certainty is unattainable, we may yet reach a degree of probability well warranting the attempt, a timid abandonment of which would be treason against all true philosophy.

Human physiology ranks as the highest department of that great science of organic life which has made such astonishing progress of late years, compassing conclusions and results which would once have been deemed impossible to human research. The closer study of comparative anatomy - the improved use of the microscope - the increased resources of chemical analysis - the wider sphere of actual observation and greater exactness in the collection and comparison of facts 
- all have concurred to this end. Other sciences, moreover, and especially geology, have lately furnished new aids to this branch of knowledge. What space is to the astronomer, time is to the geologist - vast beyond human comprehension, yet seen and comprised by the conclusions of the science. The astronomer indeed throws his line of numbers more boldly and securely into the depths of the infinite before him. The geologist can rarely give this mathematical certainty to his subject, or express the vastness of time more definitely than by the relation and succession of periods. But this result, and the methods by which it is attained, are such as well attest the grandeur of the science. The study of fossil remains, in representing successive epochs of change, and renewed creations of organic life on the surface of the globe, becomes the interpreter of facts of transcendent interest. What more wonderful than to extricate from the depths of the earth those mute yet expressive evidences of time far anterior to the creation of Man! - of ages to which no human estimate can ascend, save as respects the mere order of succession in the series! What nearer material approach can man find to his Maker, than in deciphering these successive modifications of animal life, which, while still including its simpler forms, gradually acquire higher types of organisation, and express a scheme of constant progress, however imperfect our view of the steps through which it is made? Dividing these periods by the geological characters which so clearly denote their relative age and succession, and the altered conditions of the earth in each, we may affirm that each period, amidst a general change of species, contains some element of higher life and more consummate organisation. We have not room to dwell on this topic, or to detail the different expressions which naturalists have given to the general fact; but its bearing upon our subject - the natural history of Man - will be obvious at first sight, and rises in importance as we pursue and enlarge the enquiry. 
For what is the position of Man in the scheme and series thus described? The answer is written in the same great volume of nature; - the evidence negative indeed in part, but not on that account less certain. While all anterior conditions of animal life, as they have successively occurred, are represented to us by innumerable vestiges and fossil remains, no trace whatsoever is found of the human being until we approach elosely the epoch of his present existence. Bones, shells, impressions of the most delicate structure, even the passing footsteps of animals over a moist surface, all these things have been wonderfully preserved to the inspection of our own age. The most minute as well as the most gigantic forms of the ancient animal world in its several periods are familiar to our present knowledge. If in one spot the remains have been too imperfect to allow the naturalist to complete his delineation, such is the rich exuberance of this fossil world that he rarely fails to obtain what is wanting from some contemporaneous or similar strata elsewhere on the globe. Even the lacunce which still exist in the series of zoological types are in progress of being filled up from the same fertile source. - Yet of Man, not one vestige is to be found among any of these earlier creations of life on the earth. A single bone, distinctly discovered in a well-marked geological site, and attested as human by Cuvier or Owen, would have decided the question. But none such of this date have hitherto come to our hands. And the creation of Man must be referred, if not to a period coincident with the last changes in the surface of the globe, yet certainly, upon present proof, to a time far later than those great revolutions which have consolidated, raised, dislocated, or otherwise altered the stratified rocks, at the successive periods of which geology furnishes the record and the proof.*

* The opinion here stated can hardly be thought discordant with the recent discovery of flint implements (evidently the work of Man) in the Chalk gravel 
Though less certain in evidence, it is reasonable to add in confirmation of this view, what we have just stated as to the introduction of certain higher organisations at each of the periods in question. The step from the most advanced genera of the Mammalia to Man may be greater than any antecedent one; but still we are not entitled to disregard this latter relation, as forming part of the great scheme which we venture to contemplate with the faculties permitted us so far to use. The mere fact that human reason is rendered capable of addressing itself to such objects, attests more strongly than any other the actual pre-eminence of Man over all besides of the existing creation.

This point then settled, as far as our present knowledge permits, we come to the particular questions regarding the first condition of Man on the earth, which we have indicated as lying at the root of the whole enquiry. Is the human being a sole species of what naturalists call the genus Homo? or do the diversities of physical character which we see in different races compel the admission that there were more species than one in the original act of creation? Again, if the unity of the species be proved, are we to look for the origin of this species in a single pair placed in some one locality of the globe, and thence diffusing the human race over its surface? or do the facts observed make it probable that there were more than one - possibly several original pairs - representing the more marked diversities of the species, and located in different points, so as to become centres of diffusion and admixture of these varieties?

or drift of the valley of the Somme and in various other localities of France and England. These indicate a period coeval with that of the Mammoths, and of several other species of Mammalia, either extinct or unknown in this climate. The same may be said of the discovery of crania and other human bones, similarly associated, in caverns in different parts of France, Belgium, Germany, \&c. These facts, duly authenticated, must necessarily modify our views as to the period of the human creation, still leaving its date as one of the latest events in the history of life on the globe. 
The questions thus propounded may be said to include all others appertaining to the subject; save one perhaps, already adverted to, but which we must here notice again, inasmuch as it involves the very definition of a species, and suggests contingencies which, if admitted, change the whole aspect of the enquiry. We allude to the opinion of those naturalists who, holding that there is no sufficient reason to suppose the immutability of species, believe it possible or probable that what have hitherto been considered such, may, by the operation of various natural causes acting through long periods of time, be gradually transmuted into other and very different forms, or species as we now regard them. On this momentous question we have spoken at length elsewhere, and need not now revert to it in detail.* We admit the fact of great changes in animal organisation, due to the progressive working of natural or artificial causes, and capable in numerous cases of becoming permanent by propagation. Further, we cannot doubt that our catalogues largely err in recording as separate species what are but varieties of the same. Still we believe that species, very numerous and widely different in their type, have an original and lasting existence in nature. However difficult to explain their number and individuality (difficulties whch occur under every theory), we see in them an intention and law of creation. The variations themselves of which species are rendered capable do, by the very limits imposed on them, and the constant tendency to recur to the primitive conditions, become an exponent and proof of such general law.

That which chiefly here concerns us is the question as to the relations of Man to the Quadrumana, and especially to those (the Gorilla, Chimpanzee, \&c.) which obviously stand next to him in the scale of the animal creation. May it be that in time, and through a succession of changes, the lower

* See the second Article in this volume, 'On Life and Organisation.' 
creature has finally reached the form and conditions of the higher? With a rare but rash intrepidity Lamarck hastens to an affirmative answer, and even denotes the manner and steps of the transmutation. On this question also we have spoken elsewhere; and need not repeat the reasons we consider valid against any such belief. We admit the proximity of these creatures in structure and in various conditions of intelligence; and we see this approach more strongly marked when looking to the inferior races of mankind. But proximity is not identity; and Man, though one member only of the animal creation, has his individuality and specific place at the head of the scale. In a mere physical sense, he stands there simply as the highest, in a long series of animal types; connected by close though perhaps unequal links, and subject to the same general laws determining the origin, distribution, and variations of species. On every principle of just classification he forms a genus to himself; and it is the conclusion of Prichard and others that this genus differs from all other genera of the animal kingdom, in containing but one species. Still we must ever hold it in view that he is a part of the great scale of animal life; and we shall speedily see how many arguments and analogies may be drawn as to what regards his physical history, from those inferior beings which exist - some for his uses, many more for his contemplation, - in the world around him.

This is especially true as respects the question to which we now come, having already in part premised our opinion upon it, viz. whether there be one species or more of the genus Man? - Whether (to put the most cogent case in front) the perfect Negro and the perfect European, seeing the strong contrasts they exhibit, can be rightly deemed of the same species? - and Whether, to explain other striking varieties in the races of men, it is needful to extend yet further this view of their specific differences? In discussing these points 
we must limit ourselves to the reasons which best explain and vindicate the conclusions obtained.

The question naturally first occurs - and it is a question which becomes an argument in itself - If Man be not a single species, how many species of the human being must we count on the earth? The Negro is the most striking contrast to the European; but the beardless yellow Mongolian also has characteristics so strongly marked, that we cannot concede the difference of species in the one case without admitting it in the other. How, or where, are we to stop in these admissions, when we find diversities similar in kind, and different only in degree, existing everywhere around us; and marking those divisions into races, of which many have retained the same distinctive characters from the earliest periods of history? This question is further perplexed by the intermixture of races and varieties; rendering it difficult, if not impossible, to define any such primitive separation of origin as the phrase of different species implies. Multiplicity then in this case becomes itself an argument for unity. No lines of demarcation are found sufficiently. strong to render the plurality of species natural or probable. Every such line is crossed by others which, while effacing its distinctness, do all point to a certain common origin - expressing in this what we believe to be the unity of the species over the earth.

This manner of putting the argument however, though strong, is obviously not conclusive. It is rendered much more forcible by a regard in detail to those conditions which may fitly be considered as showing the identity or diversity of species; and further, by analogies derived from the variations of species in other parts of the animal creation. From these two sources, concurring in their evidence, we derive conclusions as certain as can be had in those parts of natural science where the proof is presumptive only. 
And first, as to the criteria which best determine the identity or diversity of species recognised as such. Limiting our present view to that part of the scale most approximate to Man, we may state the following conditions as those to which we must apply for solution in each particular case ; viz. the anatomical structure in all its parts; - the average duration of life; - the relation of the sexes and laws of propagation, including the periods of utero-gestation and number of progeny; - the production, or otherwise, of hybrid progeny by mixed breeding; - the liability to the same diseases ; - and the possession of the same instincts, faculties, and habits of action and feeling. It will readily be admitted that wherever individuals or groups of beings concur as to all these general conditions, there the proof of identity of species is complete. But we have already spoken of that capacity for variation within certain limits in each species, which may as justly be called a law of nature as the division into species itself; and we are in no instance entitled to expect entire conformity to the several conditions stated above. In recurring to them hereafter it will be seen that each condition includes a liability to such variations, more or less, for every species; and it would seem that this increases as we rise upwards in the scale of animal life. In the higher animals, and notably in man himself, this capacity for variation shows itself peculiarly in all that regards the instincts, habits, and mental faculties, as modified by climate, food, culture, and other contingencies. In the phenomena more strictly of physical organisation, a lesser amount of change is likely to occur; yet here also (and it is an important point in the argument) the familiar experience of every one will indicate innumerable such varieties, more striking as the research is more extended and minute.

Taking these circumstances into account, our demand for proof of the identity of species need not go beyond a general 
conformity to the conditions above stated; - with the admission in each instance of a certain amount of deviation from the common characters; but the deviations themselves alike in kind under like conditions, and prone to return to this primitive standard when the causes of change are removed. Submitting the case of the human being to these criteria, which have helped to solve many doubtful questions as to other species, we may confidently say that an affirmative answer is derived from all as to the proper unity of Man. In truth each point has been directly or silently conceded, except those which regard on the one hand, configuration, colour, and certain other bodily peculiarities, and on the other the equality of the mental endowments and capacities. On these points discussions have been raised; and the persuasion stated by some writers, that the corporeal and mental diversities of the Negro and Caucasian cannot be explained otherwise than by supposing a difference of species; - thus sanctioning the unwarranted notion which the ignorant or the interested have so often adopted as to this matter. It may be doubted whether this opinion has now many formal advocates; and we might not think it needful to dwell further on the argument, were it not that the reasonings apply almost equally to that modified view, which, without denying the identity of the species, affirms that there were different pairs of different primitive races, placed separately on the earth. Every argument, of course, which tends to show that one species is capable of undergoing the variations actually found among mankind, must apply pro tanto to this latter doctrine also.

Let us first look at the anatomical part of the question. The characters most dwelt upon in the discrimination of the different races of men are the skeletons, and particularly the skull and pelvis - the stature - the colour of the skin - and the texture of the hair. In all systems of arrangement of 
these races, the figure of the skull has formed a principal feature; the differences in this structure (so important from the organ it encloses) being such as are obvious to the most careless observer. The early researches of Camper and Blumenbach on this subject have been much extended since their time. The collections of Retzius of Stockholm, and of Warren and Morton in the United States, well known to us by personal inspection, have much augmented our acquaintance with the crania of different races over the globe. These new acquisitions have often proved important in furnishing links between cranial forms widely dissociated before: - they have shown nothing which can be construed into a diversity of species. Nevertheless the varieties of crania are strongly enough marked to justify a division founded on this character, though naturalists have not hitherto agreed in that which should be adopted. The one originally proposed by Blumenbach included five races the Caucasian, Ethiopian, Mongolian, Malayan, and American - and this with little modification was long acquiesced in. The later researches of Dr. Prichard, founded on more ample materials, led him to reduce the chief types of cranial form, and the distinction of races founded upon them, to three only. These he characterises from their several peculiarities, as the prognathous, the pyramidal, and the oval or elliptical. The prognathous, or that marked by the predominance of the jaws, is the cranial type of the lower Negro and Australian races; - the pyramidal crania, connected with broad, lozenge-formed faces, furnish a type common to the Mongolian or Tartar nations, the Laplanders, Esquimaux, Hottentots, and many American tribes; - the oval or elliptical cranium expresses the form common to the Caucasian races and all the more highly civilised nations of the world.

While acquiescing in this division, we do so simply from its being the one most natural and comprehensive, where 
some such is required for the elucidation of the subject. Under the view we entertain that the distinctions of cranial form (endlessly multiplied in their varieties, both in races and individuals), are secondary and derived from a common source, we can attach no higher importance than this to the classifications proposed. Our present knowledge enables us to follow these more strongly marked types into each other through all the intermediate links. We can even go farther, and affirm that some of the changes in question are taking place under our own eyes. The Turks of Europe and Western Asia are doubtless of the same stem as the Turks of Central Asia; yet they have gained, probably within a few centuries, the cranial form and facial features of the Caucasian races; while those retaining their original seat and manner of life retain also the pyramidal skull and Mongolian characters of the race. The Laplanders, Finns, and Magyars, - all derived, as we have reason to believe, from the Mongolian stock - present three gradations of change from the pyramidal to the elliptical type, and bearing proportion to the degree of civilisation attained by each. Again, we have various testimony that the Negro head, so strongly marked in its characters, is gradually coming nearer to the European form, where successive generations of Negroes, without any actual intermixture, have lived in constant communication with this higher race.

As a particular feature of the cranium, the facial angle (determining the relation of the line of the forehead to that of the face), is a subject of interest even to the most common observers, in its seeming connection with the intellectual developement and expression. Its great diversity in different individuals is well known; and the same variation, within certain limits, extends to different races. Naturalists have busied themselves in giving exact measurement to this angle both in man and the inferior animals; and with results 
which at first were held by not a few of them to sanction the idea that the Negro was an inferior species, and descending nearer in this part of his developement to the Anthropoid apes. But more exact researches have corrected various errors in these results, both as regards the monkey and the man; degrading the former from his acquired rank, and restoring to the latter his identity with the rest of the human species. In truth, the average diversity in this part of the cranial form in the Negro is far below the occasional deviations of the same kind in the European; and both must be regarded as effects of that general law of variation, which shows itself alike in individuals, in families, and in races of mankind. The value of the last remark will be manifest as respects both this particular topic and all other parts of the question.

What we have just stated respecting the diversities of the skull in different races, and the inferences therewith connected, will exempt us from saying much as to the other anatomical points in the question. The form of the pelvis, the length of the fore-arm, the position of the head in reference to the vertebral column, as well as the colour of the skin and character of the hair, have all been cited in proof of a specific difference between the Negro and European stock, and the closer relation of the former to certain species of the Quadrumana. But the argument has been disproved in each case; - partly by enlarged enquiry, as in the instance of Professor Weber's valuable researches on the pelvis; - partly by more exact admeasurements and the application of that system of averages which has contributed so greatly to the progress of science; - partly, again, by the considerations we have already propounded as to the varieties naturally incident to the same species; the graduation of all these varieties into each other; and the occurrence of the same or larger deviations in individuals or families as 
in races of men. Take, for example, the colour of the skin, to which the latter class of arguments chiefly applies, and the diversities of which are at least as prominent as those of figure. The extreme contrasts in this case are the Negro and the Albino. The latter is clearly an accidental variety; but, as such, becomes from its marked characters a valuable exponent of all other varieties of colour. That part of the structure of the skin, which is called the pigment-cell, is evidently capable of undergoing great changes in its secretions from climate, manner of life, and those more mysterious causes connected with generation and the hereditary transmission of bodily features and peculiarities, the influence of which we everywhere see, but which we cannot yet subject to specific laws. Time is manifestly an element of great importance here. The amount of change of which we have evidence, even within short periods, is the proof of the capacity for far greater change where time is prolonged, and a community of men so placed as to be exposed continually to the operation of the same physical causes.

When to these considerations we add the facts upon which we have already so much dwelt, viz. that nature produces frequent varieties in all races as striking as are the extreme diversities amongst them; and 2 ndly, that there is an entire continuity in the gradations which occur in nature from one diversity to another, we present the argument in the most complete form it can assume. Thus, to take a single but striking example of the first case. A Negro may have an Albino offspring without pigment-cells; - a fact including at once all those minor varieties of colour which are so familiar to us in the same community, and even in the same family. The continuous gradations of colour from the Negro to the native of northern Europe, though less obvious to common knowledge, have been so well substantiated by travellers and men of science, that no remaining doubt can 
exist on the subject. The same two methods of argument (of which we are anxious to convey the full value) apply equally to the hair of the Negro; which, though called woolly, has no relation to wool, and is found to graduate through a series of changes into the ordinary hair of the European races in one or other of the many varieties which these races present.

The argument for the unity of the human species might perhaps be sufficient, even if it ended here. But it is exceedingly strengthened from a source to which we have more than once alluded, viz. the analogies presented by the inferior species of animal life. We have already said that Man physically considered (and it must be added intellectually also) is subject to be thus questioned by analogy; and this is pointedly true in the great question of species and varieties. The exuberance of this topic is such that we can give but a slight indication of it here. Those who desire to pursue it further will find ample means of doing so in the numerous works on Natural History, Physiology, \&c., which have lately appeared.* The main point in the argument is this; - that other species, and notably the races of domesticated animals, exhibit varieties precisely of the same kind as those occurring in mankind; - often more extensive in degree, and in many cases derived from similar causes. The outline of this argument, as applied to the horse, the dog, the ox, the hog, the sheep, the domestic fowl, \&c., will be understood by every one. We know, and regard without surprise, those vast diversities of size, figure, colour, habits of life, and even instincts of action, which distinguish the various breeds of these animals; separating them all more or less from what we may regard as the original stock of each

* Without any undue preference, we would refer to the copious writings of Dr. Carpenter on these subjects, as distinguished by great ability, and by very exact knowledge brought down to the most recent time. 
species. It is only indeed in certain instances that this primitive stock can be found amidst the varieties that have been impressed upon it. The best evidence is often that of reversion to the original form, where the artificial conditions of domestication are altered or withdrawn.

Selecting one instance in illustration, let it be the Dog; that singular animal, which Cicero well affirms to be created for the especial uses of man. What is there in the diversities of the human species comparable to those which this animal exhibits in size, in the form of the muzzle and cranium, in the colour, quality, and quantity of its covering, in the sounds it utters, in its intelligence and habits of life? What more different in aspect than the bull-dog, the Newfoundland dog, the Cuba dog, the pug-dog, and the greyhound? Yet we canot reasonably doubt (the dog itself, whatever its race, certainly does not doubt) the identity of the species. A question still exists among naturalists whether we may consider the wolf or jackall its original type. This point, however, does in no way affect the general argument. What concerns us here is the amount of variation of which the species is capable, and the varieties actually produced by nature or culture; and very especially by the intimate connection of the dog with the uses, habits, and affections of man. These are the illustrations we seek for, and they are abundantly furnished. They indicate not merely the changes which are brought about in the individual, but still more remarkably those which are transmitted to offspring, and become hereditary in its breeds. Going beyond this again, we find proof in the history of the same animal (made known to us even from mummies discovered in the tombs of ancient Egypt), of there being a limit affixed to these deviations from a primitive type. And we have further evidence that where dogs are removed from the homes and influence. of man they lapse again into a wild state, assume a form and 
colour distinct from that of their domesticated state, and often even lose the power of barking, which some have supposed to be an acquired quality not natural to the species. The dingo of Australia and the dhole of India are instances of this wilder and more primitive state. The wolfish dogs which haunt the streets of Constantinople and other eastern cities, furnish more familiar examples to the same effect.*

Similar illustrations might be drawn from other domestic animals, but less striking as they are less closely associated with man. They all offer examples of the remarkable facts to which we have alluded as a main element of the varieties of race; - those, to wit, which show the transmission from one generation to another of qualities or instincts artificially acquired; and which so transmitted, and maintained by use, tend to become hereditary in the breed. The extent to which this capacity for change proceeds - the relative permanence of the changes so induced-the parts of structure or functions most liable to them - the conditions favouring or limiting them - these are all questions infinitely curious and largely open to future research. They are connected closely, moreover, with the history and theory of analogous variations in Man; - the manner of operation being similar, and the extent and limits of deviation defined by the same general laws. In these domestic species more especially, from the manner in which certain acquired qualities become hereditary in particular breeds, we obtain an index to the formation of races among mankind. The enquiry, so conducted, gains in value and importance when we reflect on its relation to the future destinies of Man; and see in this power of transmission of acquired faculties, the possible element of new and higher conditions of our own species. There is nothing even improbable in this view, when we regard the various conditions

* Written at Constantinople (1849) with this strange canine commonwealth under my eye at the time. 
of human life existing around us. With every allowance for the thousand causes ever controlling or retarding their influence, it seems certain that the higher grades of intellectual and social existence continually tend in themselves to still higher elevation. That some part of this effect is due to hereditary transmission (a simple name for a most mysterious fact) we cannot refuse from analogy to believe. We are further called upon indeed, both by reason and analogy, to admit a line of ultimate limit to all such deviations, - assigned doubtless to us, as to other forms of created beings, by the great Creator of all. But in our ignorance where this limit lies, such admission does in no way interdict the effort to make the nearest approach to it which the human faculties render possible.

We have hitherto spoken only of those physical conditions of the human being, by which we consider the unity of the species to be vindicated, and which go yet further to render probable the derivation of the whole from a single stock. We must not let the argument stop here. The proof rises in value and certitude as we admit the intellectual and moral endowments of man into the question. It is very true that from this source as well as from physical configuration, arguments have been derived and strongly insisted upon, by those who maintain the specific inferiority of certain races. The mental faculties of the Negro in particular have been placed in pointed contrast with those of the European; and the inference thence drawn that the former is incapable of reaching the intellectual standard of the latter, or an equal grade of social life. The adrocate for identity of species has been called upon to produce instances from the Negro race of any high attainments in civilisation, literature, or philosophy; and in default of these, summary judgement has been taken against the whole race in question.

On a subject of this kind, however, we must not be governed 
by mere words, however sanctioned by common use. The term civilisation is one of those vague generalities often applied for convenience or fashion, with very slender warranty of facts. How frequently is it defined and tested by conditions belonging to our own usages, while totally inapplicable to other climates or different circumstances of life! We talk much of civilised Europe, and, as matter of general comparison, the expression may be justified. But we must not neglect the fact, that there are districts in England, Ireland, and France, which hardly rank in real civilisation above some of the Negro communities of Soudan. If we go into the great cities of the United States, New York and Philadelphia, a comparison between the free negro population and the quarters peopled by Celtic emigrants would, we venture to say, be decidedly to the advantage of the former. We are asked for examples of some eminent advancement in literature and science. Even were the demand reasonable on other grounds, seeing the condition under which the Negro has hitherto been placed, we should meet it by asking for similar examples, of native growth, among the forty millions of wclavonian races who people the vast plains of European Russia? We might variously multiply instances to the same effect, but we prefer resting the case upon what we believe to be an assured fact, viz. that where Negro communities have been associated with European races through a series of generations, their capacities and habits become altered and enlarged, and their attainments approach closely to those of the same class in the most civilised countries. This corresponds with what we before noticed as to certain changes taking place in bodily configuration under similar circumstances. It is an example, moreover, of the variations incident to every race of mankind as well as the Negro, whenever the more essential conditions of life are altered for long successive periods of time. And as such it is very instructive in relation to our subject. 
These variations, we are bound to add, are not always of advancement alone, but sometimes manifestly of degradation from the standard of the particular race. As such we may probably regard the Hottentots and Bushmen of Southern Africa; the Esquimaux, Laplanders, and Samoyedes of the arctic circle; the Fuegians, Papuas, and numerous other tribes scattered over the globe. This fact indeed, applying: alike to the mental and bodily organisation, is one which binds itself closely and necessarily with all other parts of our argument. Those varying conditions of existence, which even in the same nation or community tend to degrade and debase certain classes, do so on a larger scale and with more lasting effect, where the insulation from the original stock is more complete, and where the circumstances of life are yet more strongly contrasted, and continued for longer periods of time.

What we have said will be readily understood as applying equally to the moral feelings and character of different races as to their intellectual faculties. The denotation of unity of origin is as strong in the one case as the other. However modified in form and expression by various conditions or necessities of life, the emotions, the desires, the moral feelings of mankind, are essentially the same in all races and in all ages of the world. We have neither room nor need for argument on this subject. History and personal experience alike concur as to the fact. Were we to cite any one instance in particular, it would be the faculty of laughter and tearsthose expressions of feeling common to all colours, races, and communities of mankind, civilised or savage; and which give proofs of identity, stronger than any reasoning - $\lambda$ óyov $\tau \iota$

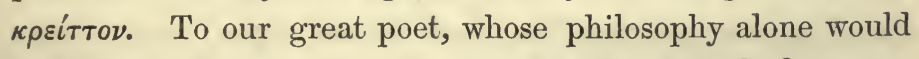
have made him immortal, we owe a line, which far more happily expresses our meaning:-

One touch of nature makes the whole world kin. 
It is this 'one touch of nature' testified in tears, which decides the question of unity of species to the common feeling of mankind as entirely as it does to the observations of the naturalist, or the reasonings of the philosopher.

Though our limits have compelled us greatly to curtail this discussion, we have pursued it sufficiently to show how much it governs the second question proposed, viz. Whether, though the species be one and single, there were not several pairs of this species placed separately on the earth?-and possibly under certain diversities of type, corresponding more or less with those of the dominant races which now exist? It will be seen that this question is already in part answered in the one preceding it; and that the grounds of argument in the two cases are closely analogous throughout. It is true, that in the latter case they are chiefly of a negative kind, and do not admit of so determinate a conclusion. We can never prove by any human evidence that it may not have pleased the Creator to give origin to the race and its varieties in this particular manner. The solution cannot be rendered other than one of probability; but we think the amount of probability such as may fairly justify the inference at which we arrive.

We are entitled, first, to ask the same question here as before; - Where is the limit to be placed to this multiplication of pairs, if intended to express the several types or varieties of man? Fischer, in his Synopsis Animalium, affirms the existence of seven forms distinct in origin. Agassiz has given the sanction of his name to the same view of a plurality of stocks. Other writers have confined them to the Caucasian, Mongolian, and Negro types; and if the doctrine of unity be abandoned, these doubtless offer the most obvious selection. But looking at the numerous varieties of mankind, and the manner in which they are 
insensibly interblended, we find no lines strong enough to form a limit to the supposed multiplicity of pairs, though many sufficiently marked to furnish a basis for the division of races. We think the evidence of facts never likely to go beyond this; and that future knowledge will tend further to confirm the belief that all these distinctions of races are subordinate to one single source of human life on the earth.

Of the arguments to this effect, beyond those already stated, the most important undoubtedly is the analogy derived from all other species of organic life. We doubt whether unequivocal proof has ever been produced of the same species, admitted as such, having even two primitive habitats on the surface of the globe. We have no means, indeed, of demonstrating the negative ; and the argument therefore must be rested on the remarkable fact, now recognised by most naturalists, that different species, whether animal or vegetable - whether terrestrial, aquatic, or atmospheric - had originally definite seats and localities on the globe, whence their various diffusion has been effected by accident or design, modified by their locomotive powers and several capacities for bearing changes of climate and place. There is now a Geography of animals and plants, as well as of mountains, rivers, and kingdoms. The importance of this discovery will at once be obvious; and not less so the great value of the facts in natural history, by which it has been established. The systematic division into provinces may undergo alterations in effect of future revision, and increased knowledge. But the general law remains good as to this primitive distribution of the forms of life, and time can only bring fresh attestation of it.

Few minds would have been hardy enough to conceive all this à priori; - to admit, for instance, the likelihood of such facts as the insulated geology and botany of the Galapagos Isles or St. Helena; or those curious relations of typical form 
in adjoining regions and on the same continents, which are observed even where the species are distinct. It cannot be doubted that geological changes in the globe, and particularly the relative changes of sea and land, have been largely concerned in the present distribution of organic life, by altering climate, and separating genera and species connected primitively with common centres. In Sir C. Lyell's work there is an admirable account of those conditions which probably have determined the various distribution of species; - closely limiting the locality of some, enabling others to occupy large tracts of the earth's surface or of the waters of the ocean. This will at once be noted as a fundamental part of the enquiry. On the one hand, while pointing at the original singleness of locality for every species, it indicates their diffusion or limitation as depending on the capacities of each for undergoing deviations which enable them to sustain changes of climate, food, and other conditions of life. On the other hand, it indicates the effect of these altered conditions of existence acting on certain parts of the animal structure and economy, and modifying them within the limits of change prescribed to each species; thus completing the circle of demonstration required.

Following, then, this great line of analogy from inferior species, we are led to infer that Man also had his origin in a single and definite place on the earth; whence he has diffused himself more widely over its surface than any other species, by virtue of those eminent faculties of mind, as well as body, which enable him to meet even the extreme contingencies of climate and food, and to adapt his existence more variously to the circumstances around him. Man can clothe himself, can fit his habitation to the climate, can prepare his food by cookery, can provide artificial means of transport, and, above all, can communicate to his fellow-men by articulate speech. In the simple expression of these 
familiar facts, common to no other animal with him, we have the history of his distribution over the globe; and can conciliate this with the belief that he had his origin in one spot alone. We have adverted to the deficiencies of history respecting the early migrations of mankind and their gathering into communities and nations. And we are obliged to admit further, that we can in no satisfactory way explain the peopling of the many remote isles of the ocean, seemingly inaccessible to Man in the ages to which such events must be referred. Still the difficulties of solution do not alter the facts to be solved. The human race is actually spread over the earth and the islands of the sea; single, as we have seen, in all that constitutes the proper definition of a species. Such is the nature of this distribution, that the difficulties are not better obviated by supposing two, three, or more centres of creation than one only. We must, in contradiction to the analogy of other animal species, make the number incalculably great, to satisfy this method of solving a case, which, after all, is reducible to probabilities perfectly justified to our reason. A more momentous and difficult question is that of the time involved in this early part of Man's history, and requisite to explain his actual dispersion and multiplication over the surface of the globe. But this question applies itself equally to all parts of the subject; - to the variations of bodily type, as well as to the local distribution of races and nations, and the growth of the many languages which have become the use of mankind. The theme, thus defined, is far too vast to be submitted to a mere outline like that on which we are now engaged. All we can here affirm is, that no positive measure of time has yet been obtained for the term of human existence on earth; but that we are bound upon various evidence to admit a longer period than any denoted in our actual chronologies; - a period which, in 
its earlier part at least, is coincident with the epoch of many forms of Mammalian life now wholly extinct on the globe.

Meanwhile, recurring to the physical evidence for the origin of mankind from a single stock, we may advert once more to the fact, that the actual deviations in Man from a common type or standard are less than those which we find in the animals most familiar to us by domestication. The causes of variation, as we have seen, are in main points the same; including that most remarkable cause, the tendency in certain acquired qualities or habits to become hereditary in the race. To this great natural phenomenon, we may trace many of the more prominent features, physical, moral, and intellectual, which distinguish races and nations. Its operation begins with individuals and families where the effects are most familiar to our observation;-widens, though becoming less marked, as these are grouped together into larger communities; - blends itself variously and closely with all the other natural causes which modify the species; - and finally, though more obscurely, forms the basis of what we call national character; a term often vaguely used, but true in itself, and involving some of the most curious questions which concern the condition and prospects of mankind. The whole subject is one fairly approachable by human reason and observation; yet hitherto less studied than we might suppose likely, seeing that these same causes are actually and constantly in operation under our eyes, shaping out new forms of national character, and with them new destinies for the human race.

There yet remains a question, and that a curious one, connected with the physiological part of our enquiry. If mankind, as now peopling the earth, be of one species, and derived from a single pair, what bodily configuration and character had this simple primitive stock? Were the 
originals of our species like to any of the derivative races, or moulded in some form now lost amidst the multitude of secondary varieties? In his earliest researches Dr. Prichard adopted a view somewhat repugnant to the common notions and feelings of the civilised world. He boldly stated his belief that the Negro must be considered the primitive type of the human race; resting this conclusion on the following grounds; - 1st, that in inferior species of animals any variations of colour are chiefly from dark to lighter, and this generally as an effect of domesticity and cultivation; 2ndly, that we have instances of light varieties, as of the Albino, among Negroes, but never of anything like the Negro among Europeans; 3rdly, that the dark races are better fitted by their organisation for the wild or natural state of life ; 4thly, that the nations or tribes lowest in the scale of actual civilisation have all kindred with the Negro race.

Taking these arguments as they are stated, and even conceding for the moment the assumptions they involve, we see no such cogency in them as to oblige us to relinquish the fairer conception of our original progenitors. Even Dr. Prichard himself seems to have abandoned this theory in his later writings, though rather by silent evasion of it than by avowal of change. While, however, we refuse on present proof to people our Eden with a negro pair, we must fairly admit that we can give no satisfactory answer to the point in question. Direct evidence on the subject is wholly wanting, nor do we see whence it can ever be obtained. There is as much reason for supposing the original type to be altogether lost, as for believing it to be represented in any one form that now exists around us. All we can presume with any degree of assurance is, that this primitive type did not depart out of the limits of existing forms, in whatever manner or proportion it may have combined their varieties. Beyond this we can affirm nothing; and rather than hazard an idle 
speculation, are willing to leave the question in the obscurity where probably it must ever remain.

We have now completed the outline of this enquiry, as far as the physiological argument is concerned. It may, we think, be considered as proved that Man is one in species, and as highly probable that all the varieties of this species are derived from one stock, and a single locality on the earth. There are no difficulties attending these conclusions so great as those which other theories involve. - And it is a further indication of their truth, that, in proportion as our knowledge in the collateral sciences has become larger and more exact, in the same proportion have these difficulties diminished or disappeared. Armed then with this strong presumption, derived from one source, we might now approach the second part of the argument; viz. that which regards the history of human languages under the various forms in which they are spread over the globe. But this subject is much too extensive to be made subsidiary only; and we can enter upon it only so far as to notice its bearing on our argument, and the general conclusion it involves.

That language should exist at all, and that it should exist among every people and community of the earth, even those lowest in the scale of civilisation, is in itself a cogent argument for the unity of Man as a species. As is the case with so many other wonders amidst which we live, its very familiarity disguises to us the marvellous nature of this great faculty of speech, confided to Man - and to Man alone - as the exponent of his intellectual and moral nature. The more deeply we look into the structure and diversities of language, the more does this wonder augment upon us; blended with great perplexity, in regarding the multitude and variety of its different forms, which though hitherto reckoned only by approximation, certainly exceed some hundreds in number. 
It is to this seeming chaos of tongues that the labours of modern scholars and philologists have been earnestly directed; with the effect not only of better connecting and classifying them, but with the higher result of giving to the study of language the character of an especial science. These labours, directed with infinite zeal and industry to the examination of those common roots and forms which denote a kindred origin, have succeeded in reducing under three great divisions - the Aryan, Semitic, and Turanian almost all the Asiatic and European languages, whether now extant, or known by record or writing only. Some do not yet admit of such affiliation; - others, again, including most of the African and native American dialects, have been so imperfectly recorded (owing in part to the want of a true phonetic system) that they have hardly yet had a place assigned to them among the genealogies of language.

The conclusion, however, to which these researches conduct us is the same already deduced from physiology, viz. that Man is of one sole species, and derived from a single primitive source on the earth. Were there more than one species, and especially were one type really inferior in kind to another, nothing would be so likely to attest this as the manner of communication of thought and feeling. Language itself would become the surest proof and interpreter of any such diversity. But its actual varieties, only partially coincident with the degree of civilisation and social advancement, offer no lines of demarcation of this kind. However great the differences (and these are ever multiplying, until checked by the embodiment of speech in writing and literature), all languages possess and manifest in their structure a common relation to the uses or necessities of the same being.*

* It is satisfactory to be able to quote the high authority of Max Müller in support of these views. He closes the volume of his Lectures, just published (1862), by stating his opinion 'that no amount of variety in the materials or formal elements of speech is incompatible with the admission of one common source.' 
We hope now to have justified the assertion with which we began this article, that there is no subject of science of deeper interest than that which regards the natural history and primitive condition of Man. Even were the questions it involves less remarkable, and less important in regard to the present and future condition of our species, the methods of argument and sources of evidence are such as may well engage and engross every rational enquirer. The evidence is drawn from all parts of creation; - from the mind, as well as from the bodily conformation of Man himself. The argument is one of probability; always tending to greater certainty, though, it may be, incapable of ever reaching that which is complete. But this is a method of reasoning well known to be compatible with the highest philosophy, and peculiarly consonant to our present faculties and position in the universe. And if ' in this ocean of disquisition fogs are often mistaken for land,' as so often elsewhere, we may at least affirm that the charts are more correctly laid down; the bearings better ascertained; and that our reason can hardly be wrecked upon this great argument, if common caution is observed in the course we pursue. 



\section{DAY USE \\ RBTURN TO DESK FROM WHICH BORROWBD \\ LOAN DEPT.}

This book is due on the last date stamped below, or on the date to which renewed.

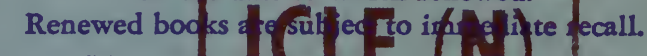

R200ct'odjex

DCT 24 ys

AUG $2413368 \%$

KECENED

AU's $10^{\prime} 66-5 P M$

LOAN DEPT:

RECD LD JAN $21 ' 69-5 \mathrm{PM}$

IN STACKS

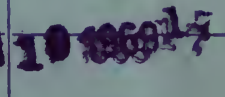

906 
YC 04955 
